DOEND-10057(92)

Distribution Category:

Limited

\title{
Idaho National Engineering Laboratory Nonradiological Waste Management Information For 1992 And Record To Date
}

V. C. Randall

A. M. Sims

Publlshed August 1993

Idaho National Engineering Laboratory

EG\&G Idaho, Inc. Idaho Falls, Idaho 83415

Prepared for the

U.S. Department of Energy

Under DOE Idaho Field Office

Contract DE-AC07-76ID01570 


\begin{abstract}
This document provides detailed data and graphics on airborne and liquid effluent releases, fuel oil and coal consumption, water usage, and hazardous and mixed waste generated for calendar year 1992. This report summarizes industrial waste data records compiled since 1971 for the Idaho National Engineering Laboratory (INEL). The data presented are from the INEL Nonradiological Waste Management Information System.
\end{abstract}




\section{EXECUTIVE SUMMARY}

The INEL Nonradiological Waste Management Information System (INWMIS) is a computerized database that replaced the Industrial Waste Management Information System (IWMIS). The INWMIS has been in place since October 1, 1991 and serves as the official record for all types of industrial (nonradiological) and mixed waste stored, disposed, or shipped from the INEL.

U.S. Department of Energy contractors routinely report data to the INWMIS. The system provides routine and special reports for nonradiological airborne and liquid effluents, fuel oil consumption, water usage, storage/disposal of solid wastes, and hazardous wastes shipped offSite. Additional reports are included in the INWMIS that are not included in previous reports. These additional reports are coal usage and generation of hazardous/mixed waste.

To more accurately report the landfill data, modifications have been made to all landfill reports to include final volumes and gross weight. Currently, the landfill data are reported by area instead of by program [i.e., Special Manufacturing Capability (SMC) and the Technical Support Facility (TSF) are reported under Test Area North (TAN)]. Future enhancements to the system will allow the landfill data to be reported by program to be consistent with all other reporting in the INWMIS.

Because of organizational changes within various groups, the reporting for the TAN area also has changed. TAN is broken into three separate sections: TSF, Containment Test Facility (CTF), and SMC. TSF and portions of SMC are currently being reported into the INWMIS.

A total of $51,658 \mathrm{~m}^{3}$ of solid industrial waste was disposed of in sanitary landfills at the INEL during 1992. This represents a decrease of $30 \%$ from the $73,823 \mathrm{~m}^{3}$ discarded in 1991 .

A total of $2,413,667 \mathrm{~kg}$ of liquid and airborne industrial waste was discharged in 1992. The industrial waste included $1,989,972 \mathrm{~kg}$ of chemicals, $267,095 \mathrm{~kg}$ of total dissolved solids, and $148,906 \mathrm{~kg}$ of total hardness discharged as liquid wastes to the lithosphere at the INEL. This waste represents a $37 \%$ decrease over the total quantity of $3,146,731 \mathrm{~kg}$ in 1990 and a decrease from the total quantity of $2,619,022 \mathrm{~kg}$ in 1990 .

The INEL used a total of $9,586,173$ liters of fuel oil during 1992 , which produced $84,861 \mathrm{~kg}$ of sulfur dioxide $\left(\mathrm{SO}_{2}\right)$ and $2,481 \mathrm{~kg}$ of particulates. During 1991, 13,802,914 liters of fuel oil were used, producing $150,721 \mathrm{~kg}$ of $\mathrm{SO}_{2}$ and $1,891 \mathrm{~kg}$ of particulates.

A total of 7,617,914,000 liters of water was pumped from the aquifer by the INEL during 1992, compared to $8,005,204,000$ liters in 1991 and $8,120,061,000$ liters in 1990. However, the majority of this water returned to the lithosphere through seepage ponds. Remaining water was lost to the atmosphere through cooling towers and other evaporatory processes.

Please note that Argonne National LaboratoryWest did not operate its cooling towers in February, April, and May because of routine reactor maintenance. 


\section{CONTENTS}

ABSTRACT $\ldots \ldots \ldots \ldots \ldots \ldots \ldots \ldots \ldots \ldots \ldots \ldots \ldots \ldots \ldots \ldots \ldots \ldots \ldots \ldots \ldots \ldots \ldots \ldots$

EXECUTIVE SUMMARY $\ldots \ldots \ldots \ldots \ldots \ldots \ldots \ldots \ldots \ldots \ldots \ldots \ldots \ldots \ldots$

ACRONYMS AND ABBREVIATIONS $\ldots \ldots \ldots \ldots \ldots \ldots \ldots \ldots \ldots \ldots \ldots \ldots \ldots \ldots \ldots$

CONVERSION FACTORS $\ldots \ldots \ldots \ldots \ldots \ldots \ldots \ldots \ldots \ldots \ldots \ldots \ldots \ldots \ldots \ldots \ldots \ldots \ldots \ldots$

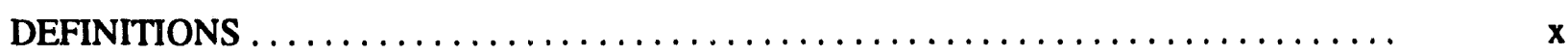

INTRODUCTION $\ldots \ldots \ldots \ldots \ldots \ldots \ldots \ldots \ldots \ldots \ldots \ldots \ldots \ldots \ldots \ldots \ldots \ldots$

TAB SECTIONS

INEL Record To Date Summaries

INEL Record To Date Detail Summaries

INEL 1992 Year-To-Date Summaries

Argonne National Laboratory-West, 1992 Detail Data

Central Facilities Area, 1992 Detail Data

Chemical Processing Plant, 1992 Detail Data

Containment Test Facility, 1992 Detail Data

INEL Electronic Technology Center, 1992 Detail Data

INEL Research Center, 1992 Detail Data

Magnetohydrodynamics Component Development, 1992 Detail Data

Naval Reactors Faciiity, 1992 Detail Data

Power Burst Facility, 1992 Detail Data

Protection Technology Idaho, 1992 Dletail Data

Potable Water Treatment Unit, 1992 Detail Data

Radioactive Waste Management Complex, 1992 Detail Data

SL1 Reactor, 1992 Detail Data

Special Manufacturing Capability, 1992 Detail Data

Test Area North, 1992 Detail Data

Test Reactor Area, 1992 Detail Data

Technical Support Facility-Test Area North, 1992 Detail Data

Technical Support Building, 1992 Detail Data 
Willow Creek Building, 1992 Detail Data

Waste Experimental Reduction Facility, 1992 Detail Data

Water Reactor Research Test Facility-TAN, 1992 Detail Data 


\section{ACRONYMS AND ABBREVIATIONS}

ANL

ARA

CFA

CFA 637

CPP

CTF

CY

EBR

HMDA

HWSF

IET

IETC

INEL

IRC

INWMIS

IWMIS

kg

L

LOF

LOFT

m

MHD
Argonne National

Laboratory-West

Auxiliary Reactor Area

Central Facilities Area

Hazardous Waste Storage

Facility (same as HWSF)

Idaho Chemical Processing

Plant

Containment Test Facility

(formerly Loss-of-Fluid Test

Facility)

Calendar Year

Experimental Breeder

Reactor-I

Hazardous Material Disposal

Area

Hazardous Waste Storage

Facility (same as CFA 637)

INEL Electronic Technology

Center (same as IETC)

INEL Electronic Technology

Center (same as IET)

Idaho National Engineering

Laboratory

Idaho Research Center

INEL Nonradiological Waste

Management Information

System

Industrial Waste Management

Information System

kilogram

liter

Loss-of-Fluid Test Facility (became CTF on October 6, 1986)

Loss-of-Fluid Test Facility (became CTF on October 6, 1986)

meter

Magnetohydrodynamics Component Development and Integration Facility (Butte, Montana)
MWSF

N/A

$\mathrm{NO}_{\mathrm{x}}$

NRF

PBF

PTI

PWT

PWTU

RWMC

SLR

SMC

$\mathrm{SO}_{2}$

TAN

TDS

TRA

TSB

TSF

WCB

WDF

WEDF

WER

WERF

WMC

WRF

WRRTF
Mixed Waste Storage Facility

not available

nitric oxides

Naval Reactors Facility

Power Burst Facility

Protection Technology Idaho

Potable Water Treatment Unit (same as PWTU)

Potable Water Treatment Unit (same as PWT)

Radioactive Waste

Management Complex (same as WMC)

\section{SL1 Reactor}

Special Manufacturing

Capability

sulfur dioxide

Test Area North

total dissolved solids

Test Reactor Area

Technical Support Building

Technical Support Facility

Willow Creek Building

Waste Engineering

Development Facility (same as WEDF)

Waste Engineering Development Facility (same as WDF)

Waste Experimental

Reduction Facility (same as WERF)

Waste Experimental

Reduction Facility (same as WER)

Radioactive Waste

Management Complex (same as RWMC)

Water Reactor Research Test Facility (same as WRRTF)

Water Reactor Research Test Facility (same as WRF) 


\section{CONVERSION FACTORS}

\begin{tabular}{llc}
\multicolumn{1}{c}{ To Convert } & \multicolumn{1}{c}{ Into } & Multiply by \\
Cubic yards & Cubic meters & 0.7646 \\
Cubic meters & Cubic feet & 35.3140 \\
Liters & Gallons (U.S. liquid) & 0.2642 \\
Kilograms & Pounds & 2.2046 \\
& DEFINITIONS
\end{tabular}

Exponential Notation-Numbers in this document are expressed as either whole numbers or in exponential notation form. For example: $1,000,000=1 \times 10^{6}=1 \mathrm{E}+06$. 


\section{INEL Nonradiological Waste Management Information For 1992 And Record To Date}

\section{INTRODUCTION}

This report summarizes nonradiological data for the Idaho National Engineering Laboratory (INEL) for 1992 and record to date. It includes summaries of the quantities of airborne and liquid effluents, the volumes of solid waste, the coal, fuel oil and water used; and the quantities of $\mathrm{SO}_{2}$ and particulates released.

These data were taken from the INEL Nonradiological Waste Management Information System (INWMIS). The INWMIS is a computerized database that replaced the Industrial Waste Management Information System (IWMIS). The INWMIS has been in place since October 1, 1991, and serves as the official record for all types of nonradiological waste stored, disposed, or shipped from the INEL.

This report provides graphics to aid in the presentation of the INEL summary data. The graphics display water, coal, chemical, fuel oil and diesel usage, volumes of solid waste discarded at the landfill, and volumes and weight of hazardous waste.

The INEL Industrial Waste Summary, a table that lists the waste data available for 1971 through 1992, is located within the tab section INEL Record To Date Detail Summaries. Also found in this section are tables that summarize INEL record to date information, and bar graphs and pie charts that provide 1992 summaries and detailed data for solid and liquid industrial waste, sanitary landfill waste, airborne and liquid industrial waste, chemical usage, fuel oil usage, water usage, coal usage, and waste oil. The graphs only show data for 1983 through 1992.

Tables that summarize INEL nonradiological waste information for the period January through
December 1992 are located in the tab section INEL 1992 Year-To-Date Summaries. This section begins with tables that summarize both the solid waste and the airborne and liquid waste generated at each INEL facility.

\section{Reports Included in Document}

INEL Record To Date SummariesThese reports summarize all industrial waste by waste type located and used on the INEL for the period 1990 to 1992 and a cumulative total for 1971 to 1989.

INEL Record To Date Detail Summaries-These reports and graphs detail the record to date quantity and type of industrial waste for each INEL facility. This section also includes a record to date report (page INEL-10) of all airborne and liquid industrial waste substances for the period 1990 to 1992 and a cumulative total for 1971 to 1989.

INEL 1992 Year-To-Date SummariesThese reports summarize the year-to-date quantity of water pumped from the aquifer and discharged and the percent of waste accounted for at each facility; the quantity and type of industrial waste for each INEL facility; the types and quantities of fuel oil used at each INEL facility; and the gross volumes and weights of hazardous waste generated by facilities associated with the INEL.

Area 1992 Detail Data-These reports summarize data on both airborne and liquid disposed substances, industrial waste, water usage and disposal, fuel oil and diesel usage, coal usage, mixed waste, and hazardous waste generated at each facility. 
INEL RECORD TO DATE SUMMARIES

INEL Industrial Waste Summary, Record To Date

INEL-3

INEL Industrial Usage Summary, Record To Date

INEL-4 
SCHED. NO. INRPTO3O

INEL INDUSTRIAL WASTE SUMMRY

RECORD TO DATE

\begin{tabular}{|c|c|c|c|c|c|c|}
\hline \multicolumn{2}{|l|}{ WASTE TYPE } & $1971 \cdot 1989$ & 1990 & 1991 & 1992 & TOTAL \\
\hline \multicolumn{6}{|l|}{ LAMDFILL MASTE* } & 725,661 \\
\hline OIL & (LITERS) & 133,912 & & & & 133,912 \\
\hline SOLVENTS & (LITERS) & 1,735 & & & & 1,735 \\
\hline $\begin{array}{l}\text { CHEMICALS } \\
\text { LIOUIDS } \\
\text { SOLIDS }\end{array}$ & $\begin{array}{l}\text { (LITERS) } \\
\text { (KILOGRAMS) } \\
\text { (CUBIC METERS) }\end{array}$ & $\begin{array}{r}132,247 \\
31,136 \\
689\end{array}$ & & & & $\begin{array}{r}132,247 \\
31,136 \\
689\end{array}$ \\
\hline $\begin{array}{l}\text { OTHERS } \\
\text { LIQUIDS } \\
\text { SOLIDS }\end{array}$ & $\begin{array}{l}\text { (LITERS) } \\
\text { (CUBIC METERS) }\end{array}$ & $\begin{array}{r}157,464 \\
223\end{array}$ & & & & $\begin{array}{r}157,464 \\
223\end{array}$ \\
\hline LIQUID EFFLUENT & (KILOGRAMS) & $67,466,944$ & $2,619,022$ & $3,370,127$ & $2,405,973$ & $75,862,066$ \\
\hline $\begin{array}{l}\text { AIRBORNE EFFLUEN } \\
\text { CHEMICALS } \\
\text { FUEL OIL ANO }\end{array}$ & $\begin{array}{l}\text { (KILOGRAMS) } \\
\text { ESEL }\end{array}$ & $4,696,021$ & 67,783 & 472,840 & 7,694 & $5,244,338$ \\
\hline $\begin{array}{l}\text { SO2 } \\
\text { PARTICULATE }\end{array}$ & $\begin{array}{l}\text { (KILOGRAMS) } \\
\text { (KILOGRAMS) }\end{array}$ & $\begin{array}{r}6,640,439 \\
434,744\end{array}$ & $\begin{array}{r}105,309 \\
4,754\end{array}$ & $\begin{array}{r}150,721 \\
1,891\end{array}$ & $\begin{array}{r}84,861 \\
2,481\end{array}$ & $\begin{array}{r}6,981,329 \\
443,870\end{array}$ \\
\hline $\begin{array}{l}\text { SO2 } \\
\text { NO2 }\end{array}$ & $\begin{array}{l}\text { (KILOGRAMS) } \\
\text { (KILOGRAMS) }\end{array}$ & $\begin{array}{l}396 \\
215\end{array}$ & $\begin{array}{r}123 \\
43\end{array}$ & $\begin{array}{r}37 \\
143\end{array}$ & $\begin{array}{l}17 \\
64\end{array}$ & $\begin{array}{l}573 \\
465\end{array}$ \\
\hline HAZARDOUS WASTE & (CUBIC METERS) & 14 & 1 & 11 & 846 & 873 \\
\hline MIXED WASTE & (CUBIC METERS) & 50 & 61 & 61 & 48 & 220 \\
\hline WASTE OIL & (LITERS) & $1,050,434$ & 111,423 & 121,504 & 53,367 & $1,336,728$ \\
\hline
\end{tabular}

* NOTE: DOES INCLLDE LAMDFILL WASTE FOR MHICH ONLY a we!ght is aVAILABLE

NOTE: SINCE APRIL 1984, THE DEPARTMENT OF ENERCY'S POSITION HAS BEEN TO ENSURE THAT ALL HAZARDOUS MASTE IS PROPERLY

MAMAGED UMDER THE RESOURCE CONSERVATION AND RECOVERY ACT (RCRA). AVAILABLE IMFORMATON ASSOCIATED UITH THE

CHEMICALS DISPOSED OF IN THE LANDFILL SINCE 1984 DOES MOT INOICATE THAT THEY WERE HAZARDOUS AS DEFIMED UNDER RCRA.

DETAILS MAY NOT ADD UP TO TOTALS BECAUSE OF ROUNDING

0 IN A COLUAM INDICATES A TOTAL OF $<0.5$ 
SCHED. NO IMRPTO3OA

INEL INDUSTRIAL USAGE SUMMARY

RECORD TO DATE

\begin{tabular}{|c|c|c|c|c|c|c|}
\hline WASTE TYPE & & $1971-1989$ & 1990 & 1991 & 1992 & TOTAL \\
\hline FUEL OIL \& DIESEL & (LITERS) & $380,263,708$ & $8,466,176$ & $13,802,914$ & $9,586,173$ & $412,118,972$ \\
\hline COAL & (TONS) & 39,960 & 12,091 & 3,291 & 12,464 & 67,806 \\
\hline WATER PUMPED & (1000 LITERS) & $161,125,468$ & $8,120,061$ & $8,005,204$ & $7,617,914$ & $184,868,647$ \\
\hline
\end{tabular}




\section{INEL RECORD TO DATE DETAIL SUMMARIES}

INEL Solid Landfill Waste In Cubic Meters, Record To Date . . . . . . . . . INEL-7

INEL Liquid Landfill Waste In Liters, Record To Date . . . . . . . . . . INEL-8

CY 1983-1992 INEL Sanitary Landfill Waste, Bar Graph And Pie Charts ..... INEL-9

Airborne And Liquid Industrial Waste Substances Summary In Kilograms,

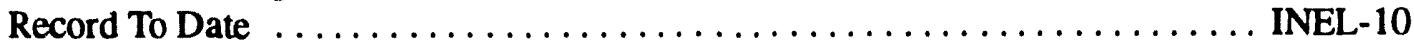

CY 1983-1992 INEL Chemical Usage (Released As Airborne Effluents),

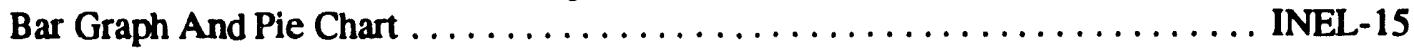

CY 1983-1992 INEL Chemical Usage (Released As Liquid Effluents),

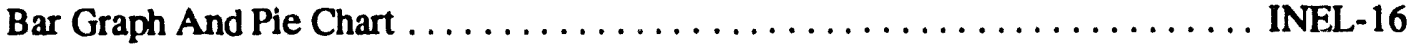

INEL Fuel Oil \& Diesel Summary, Record To Date $\ldots \ldots \ldots \ldots \ldots \ldots \ldots$ INEL-17

CY 1983-1992 INEL Fuel Oil \& Diesel Usage, Bar Graph And Pie Chart . . . . . INEL-19

CY 1983-1992 Sulfur Dioxide Emitted, INEL Fuel Oil \& Diesel

Usage, Bar Graph And Pie Chart . . . . . . . . . . . . . . . . . . INEL-20

CY 1983-1992 Particulate Emitted From INEL Fuel Oil \& Diesel

Usage, Bar Graph And Pie Chart . . . . . . . . . . . . . . . . . . . INEL-21

INEL Water Usage Summary In Thousand Liters, Record To Date . . . . . . . INEL-22

INEL Water Pumped Sumruary In Thousand Liters, Record To Date $\ldots . \ldots \ldots$ INEL-23

CY 1983-1992 INEL Water Usage, Bar Graph And Pie Chart . . . . . . . . . INEL-24

CY 1992 INEL Water Usage, Pie Charts $\ldots \ldots \ldots \ldots \ldots \ldots \ldots \ldots \ldots$ INEL-25

INEL Coal Summary, Record To Date $\ldots \ldots \ldots \ldots \ldots \ldots \ldots \ldots \ldots \ldots \ldots \ldots$ INEL-26

CY 1987-1992 INEL Coal Usage, Bar Graph $\ldots \ldots \ldots \ldots \ldots \ldots \ldots \ldots$ INEL-28

CY 1987-1992 Sulfur Dioxide Emitted From INEL Coal Usage, Bar Graph . . . INEL-29

CY 1987-1992 Nitric Oxide Emitted From INEL Coal Usage, Bar Graph . . . . . INEL-30

INEL Waste Oil Summary In Liters, Record To Date . . . . . . . . . . . INEL-31 
SCHED. MO. IMRPT035

INEL SOL IO LAMDFILL MASTE IN CUBIC METERS RECORD TO DATE

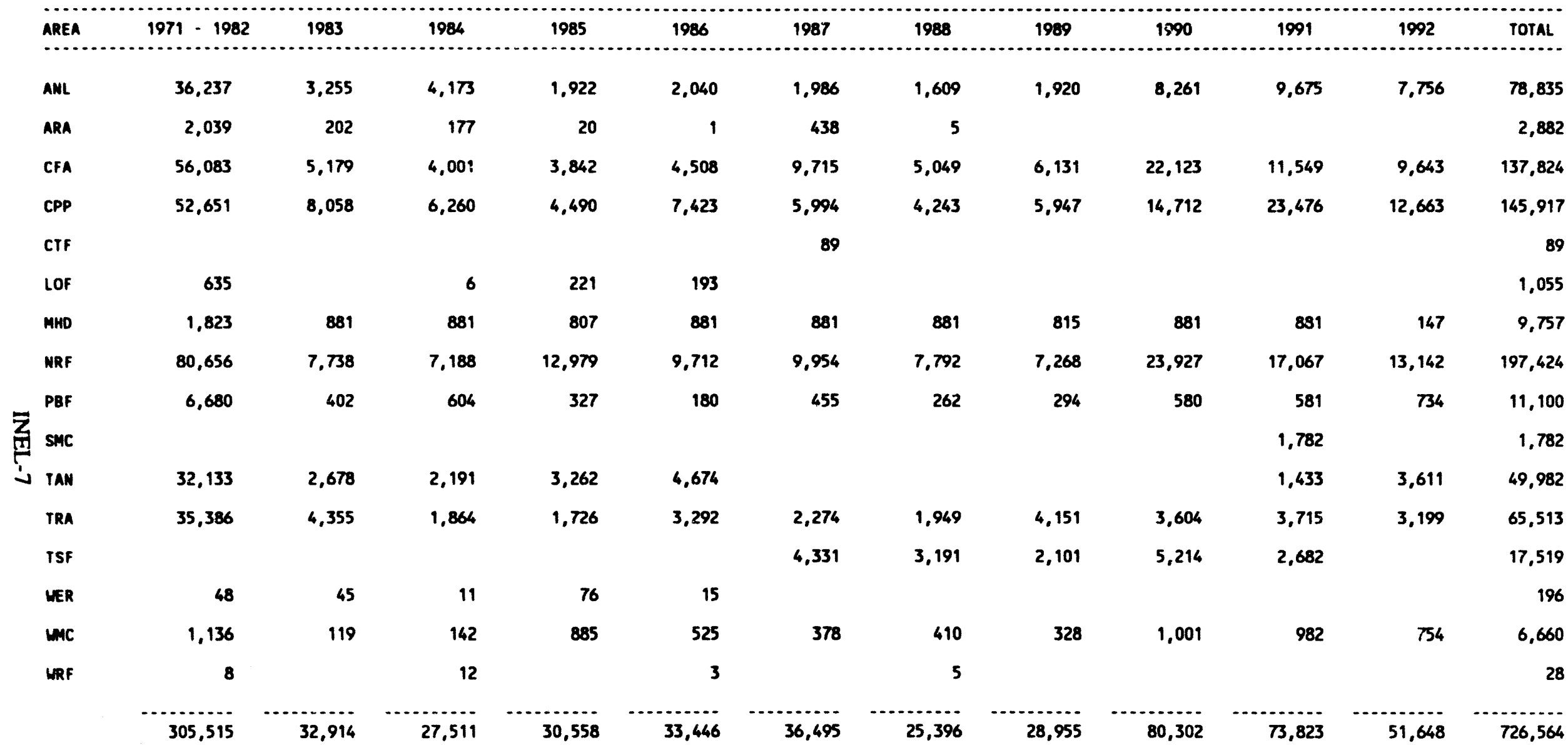

MOTE: DOES NOT INCLUDE LANDFILL MASTE FOR MHICH ONLY A MEIGHT IS AVAILABLE.

THIS COMPRISES 31,136 KILOGRAMS FOR THE YEARS 1971 - 1988.

$O$ IN A COLUMN INDICATES A TOTAL OF $<0.5$ 
SCHED. NO. INRPTO35A

INEL LIQUID LANDFILL MASTE IN LITERS

RECORD TO DATE

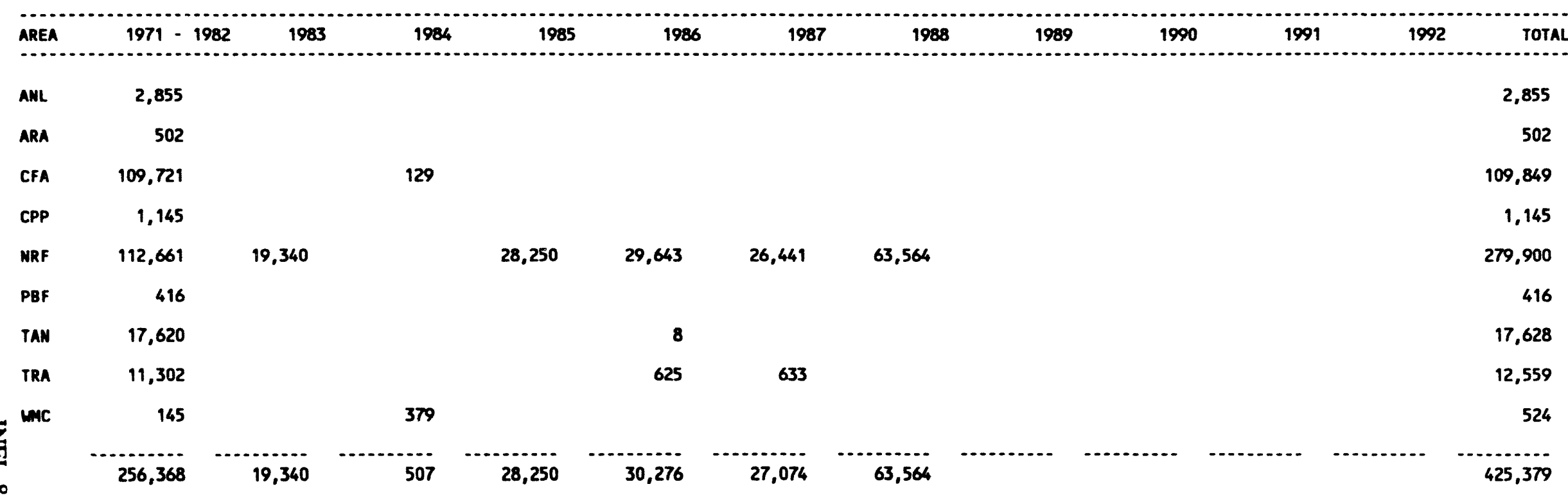

NOTE: INCLUDES ALL LANDFILL MASTE DENOTED BY LITERS ON THE INEL INDUSTRIAL WASTE SUMMARY REPORT 


\section{CY 1983 - 1992 INEL SANITARY LANDFILL WASTE \\ CY 1992 INEL WASTE BY TYPE}

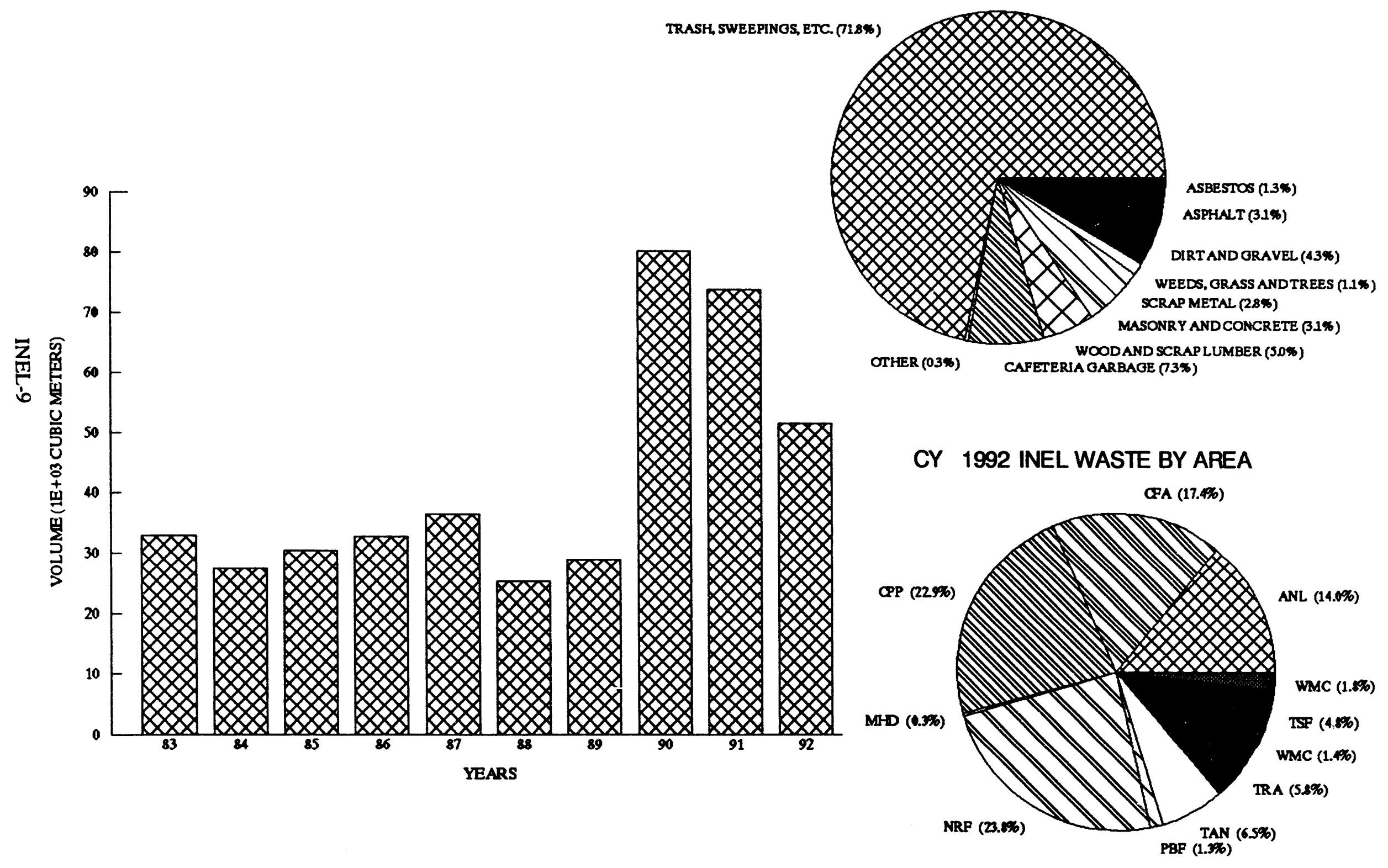


SCHED. MO. INRPT036

AIRBORNE AND LIOUID

INDUSTRIAL WASTE SUBSTANCES SUMWARY IN KILOGRAMS RECORD TO DATE

SUBSTANCE IDENTIFICATION

$1971-1989$

1990

1991

1992

TOTAL

(1- HYDROXYETHYLIDENE)BI SPHOSPONIC

1-BROWO-3-CHLORO-5,5-DIMETHYLHYDANT

1-BROWO-3-CHLORO-5, 5-DIMETHYLHYDAWTOIN

2-(2-BUTOXYETHOXY)-ETHANOL

ACETIC ACID

ACRYLIC POLYMER

ALKYL DIMETHYL BEMZYL AMAONIUM CHLORIDE

ALUMIMUM NITRATE

AMERPLEX 625

AMIDES

AMINEMETHYLPROPANOL

AMMONIA

AMTONIA

ARSENIC

ARSENIC

B-BROWO-B-NI TROSTYRENE

BARIUM SULFATE

Z BENZOTRIAZOLE

BERYLLIUN

BETZ 109

BETZ 194

BETZ 20K

BET2 406

BET2 429

BET2 45

BET2 65

BET2 736

BET2 A-9

BET2 DE 419

BETZ DE 453

BET2 DE 481

BETZ DIANODIC 194

BET2 FERROSPERSEHP

BET2 J-12

BETZ NEUTRMEEN

BIOCIDE D-2

BIS (TRICHLOROMETHYL) SUL FONE

BLEACH

BORON

BROAINE

CADMIU

CALCIUM CHLORIDE

CALCIUA FLUORIDE

CALCIUN ION

$\begin{array}{rr}44 & 472 \\ 12 & 2 \\ & \\ 182 & 40 \\ 1,547 & 49 \\ 36 & \\ 36 & 135 \\ 1 & 18 \\ 27,038 & 17 \\ 775 & 0 \\ 581 & 157 \\ 0 & \end{array}$

4,183
686
427

1,479
953

5,661

1,991

60

20

260

18
17

0
157

$\begin{array}{rr}0 & \\ 35 & \\ 301 & \\ 18,953 & 9,015\end{array}$

9,015

130
5.172

5,172

149
544

544
9

2.675

2,675
113

113
7,879

828

425
18

1,669

9,641

898

11,986
167

18

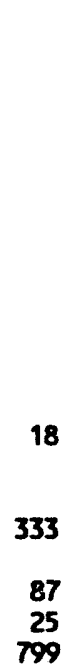

24,274

87
25
799

DETAILS MAY MOT ADD UP TO TOTALS BECAUSE OF ROUNDING

O IN A COLUAN INDICATES A TOTAL OF $<0.5$ 

RECORD TO DATE

SUBSTANCE IDENTIFICATION

1971 - 1989

1990

1931

1992

TOTAL

\section{CALCIUM MITRATE}

CALCIUN PERCHLORATE

CALCIUM PHOSPHATE, TRIBASIC

CALCIUM SULFATE, ANHYDROUS

CARBOHYDRAZIDE

CARBONTHIOCYANATES

CARBOXYALKYLATED POLYSACCHARIDE

CARBOXYLATED LIGNIN

CHLORIDE ION

CHLORINE

CHLOROCARBOWS

CHLOROHYDROCARBOWS

CHLOROSULFONES

CHROMATE ION

CHROMIUM

CITRIC ACID

CLOROHYDROCARBOWS

CR+6

CYCLOHEXYLAMINE

DIANODIC 194

DIETHYLAMINOETHANOL

DIMETHYLAMINE

DIMETHYLFORMAMIDE(DMF)

DIMETHYLI SOPROPANOLAMINE

DIPHOSPHONATE

DISPERSANT 403

DODECYLGUANIDINE HYDROCHLORIDE

DREMGARD

ETHYL ALCOHOL

ETHYLAMINE

ETHYLENEDIAMINE TETRAACETIC ACID

FLUORIDE ION

FORMIC ACID

MEAVY AROMATIC MAPTHA

MEDAVALENT CR

HYDRAZINE

HYDROCARBON-AMINES

HYDROCARBONS

HYDROGEN CHLORIDE

HYDROXYCARBONS

HYPOCHLORITE

HYPOCHLORITE ION

IMPURITY

\begin{tabular}{|c|c|c|c|c|}
\hline & $\begin{array}{r}132 \\
3 \\
19 \\
1\end{array}$ & $\begin{array}{r}34 \\
4 \\
28 \\
1\end{array}$ & $\begin{array}{r}26 \\
4 \\
28 \\
1\end{array}$ & $\begin{array}{r}192 \\
12 \\
75 \\
3\end{array}$ \\
\hline 271 & 10 & 3 & & 283 \\
\hline & & & $\begin{array}{l}1 \\
0\end{array}$ & $\begin{array}{l}1 \\
0\end{array}$ \\
\hline $8,501,098$ & 712,280 & $1,112,969$ & 722,131 & $11,048,479$ \\
\hline & & & & 21 \\
\hline 2 & & & & 2 \\
\hline 472 & & & & 472 \\
\hline 1.212 & 39 & 11 & & 1,263 \\
\hline 327 & & & & $\begin{array}{l}327 \\
128\end{array}$ \\
\hline $\begin{array}{r}120 \\
91\end{array}$ & & & 9 & $\begin{array}{r}128 \\
91\end{array}$ \\
\hline 0 & & & & 0 \\
\hline 1,815 & & & & 1,815 \\
\hline 243 & 265 & 963 & 10 & 1,481 \\
\hline $\begin{array}{r}15,531 \\
24\end{array}$ & 27 & 96 & & $\begin{array}{r}15,531 \\
147\end{array}$ \\
\hline 2,131 & & & & 2,131 \\
\hline & 17 & 4 & & 21 \\
\hline 36 & & 20 & 16 & 72 \\
\hline $\begin{array}{l}13,249 \\
13,026\end{array}$ & & & & $\begin{array}{l}13,249 \\
13,026\end{array}$ \\
\hline & & 510 & 471 & 981 \\
\hline 116 & & & 47 & 163 \\
\hline & 19 & 193 & 203 & 415 \\
\hline 15 & 3 & 2 & 2 & 23 \\
\hline 323 & 125 & 132 & 104 & 684 \\
\hline $\begin{array}{r}14,204 \\
5\end{array}$ & 473 & 330 & 482 & $\begin{array}{r}15,489 \\
5\end{array}$ \\
\hline & 13 & 29 & 21 & 62 \\
\hline 9,819 & 1,371 & 4 & & 11,193 \\
\hline 2,631 & & & & $\begin{array}{l}2,631 \\
4,415\end{array}$ \\
\hline & & & & 24 \\
\hline 759 & 9 & & & 768 \\
\hline 2,705 & 99 & 26 & & 2,830 \\
\hline 10 & 48 & 57 & 52 & 167 \\
\hline 18,659 & 243 & 8 & & 18,910 \\
\hline 144,780 & 1,397 & 2,452 & 1,776 & 150,404 \\
\hline
\end{tabular}


ISOPROPANOL

ISOPROPYL ALCOHOL

JANI TORAL SUPPLIES

JANITORIAL SUPPLIES

KP4O COOLANT CNTRL

KWIK KLEEN

LAUNDRY PRCOUCTS

LEAD

M-60 ALGAECIDE

M-80T ALGAECIDE

MERCURY

METHBISTHIOCYAMATE

METHYLEME BIS(THIOCYAMATE)

MOGUL PC 1222

MOLYBDATE SALT

MORPHOLINE

N-HYDROCARBO

W-METHYLPYRROL IDONE

MALCO 7312

MALCO 7323

MALCOOL 7351

MAPTHA

NITRATE ION

MITRIC OXIOE

NITRITE ION

NITRITE ION

NITRITE SALT
NITROGEN DIOXIDE

NITROGEN DIOXIDE
NONYLPHENOXYPOLY (ETHYLENEOXY)ETHANO

NONYLPHENOXYPOLY(ETHYLENEOXY)ETHANO

NONYLPHENOXYP
OROCOL 194

OROCOL 734

OXYCARBONS

PHOSPHATE

PHOSPHATE ION

PHOSPHONATE

PHOTO LAB CHEMICAL

POLYACRYLATE

POLYACRYLATE ACID

POLYALKYLENE GLYCOL

POLYHYDROXYCARBON

POL YMETHACRYLATE

POLYNOOIC 606

POLYNODIC 633

POLYPHOSPHATE

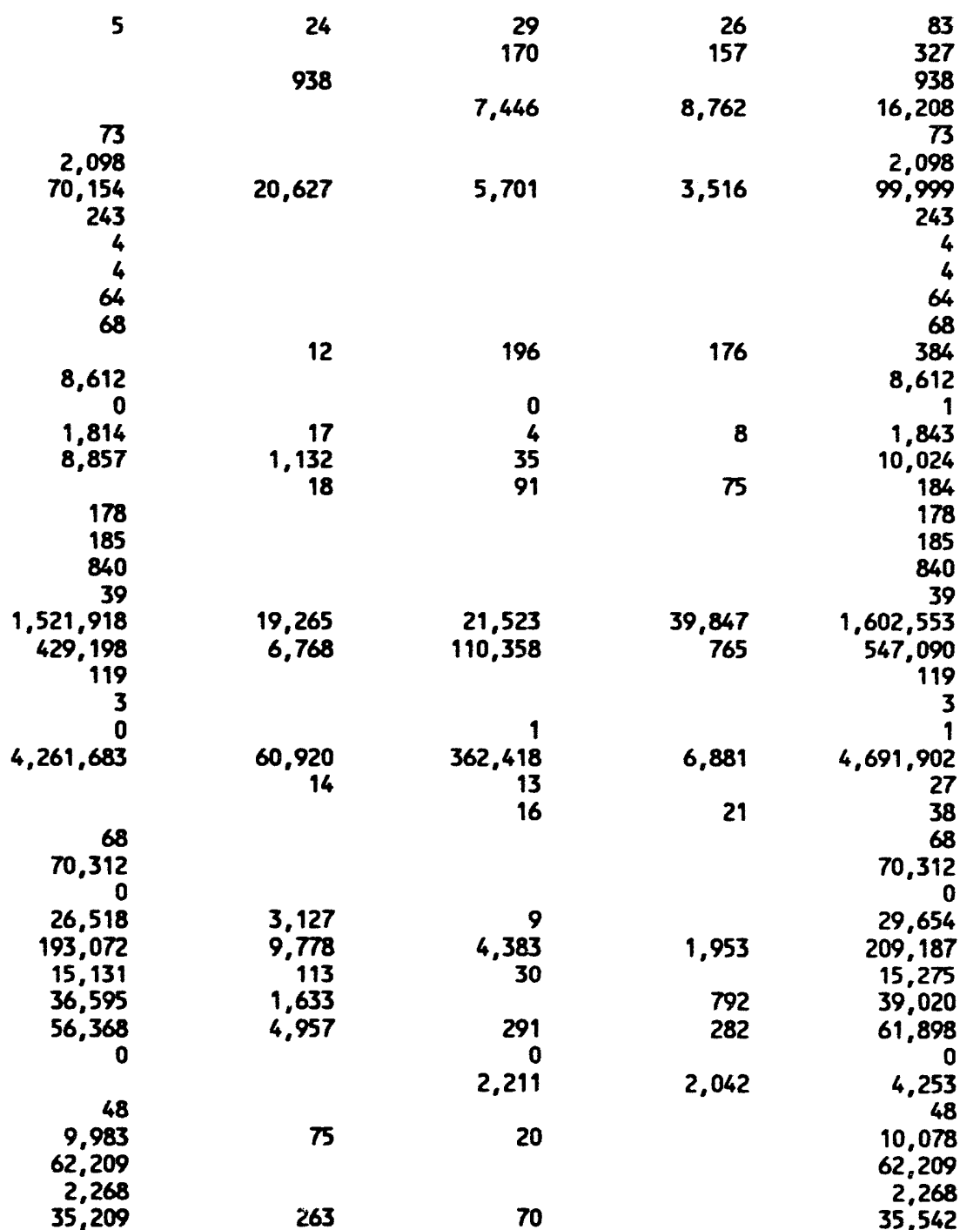


RECORD TO DATE

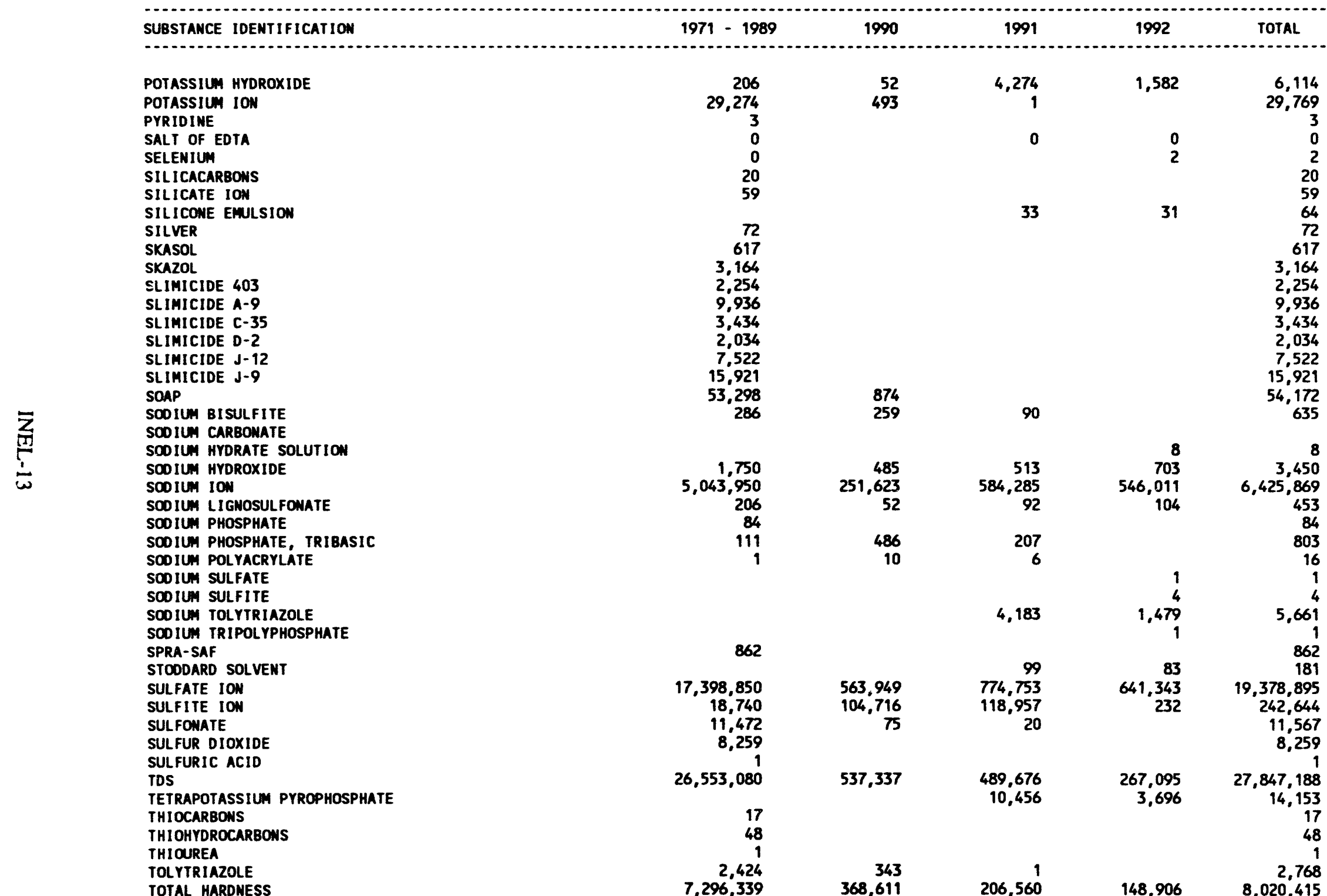


SCHED. NO. INRPT036

AIRBORNE AND LIOUID

INDUSTRIAL WASTE SUBSTANCES SUMMARY IN KILOGRAMS RECORD TO DATE

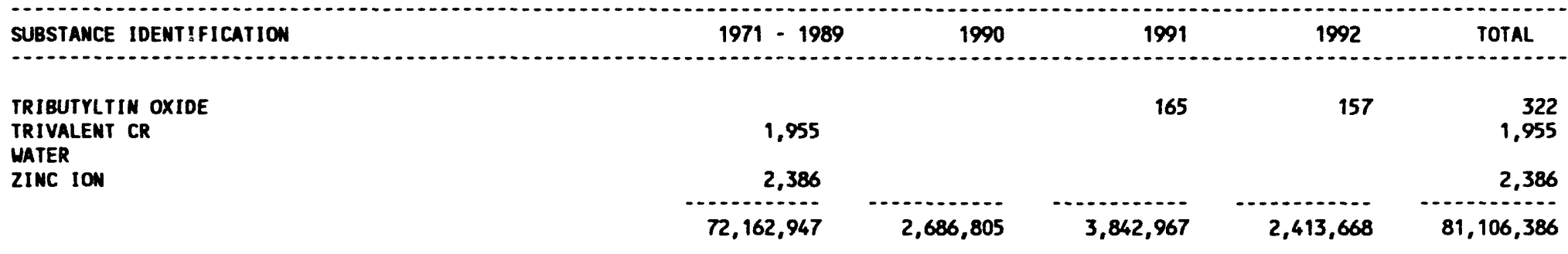




\section{CY 1983 - 1992 INEL CHEMICAL USAGE (RELEASED AS AIRBORNE EFFLUENTS)}

CY 1992 INEL CHEMICAL USAGE BY AREA

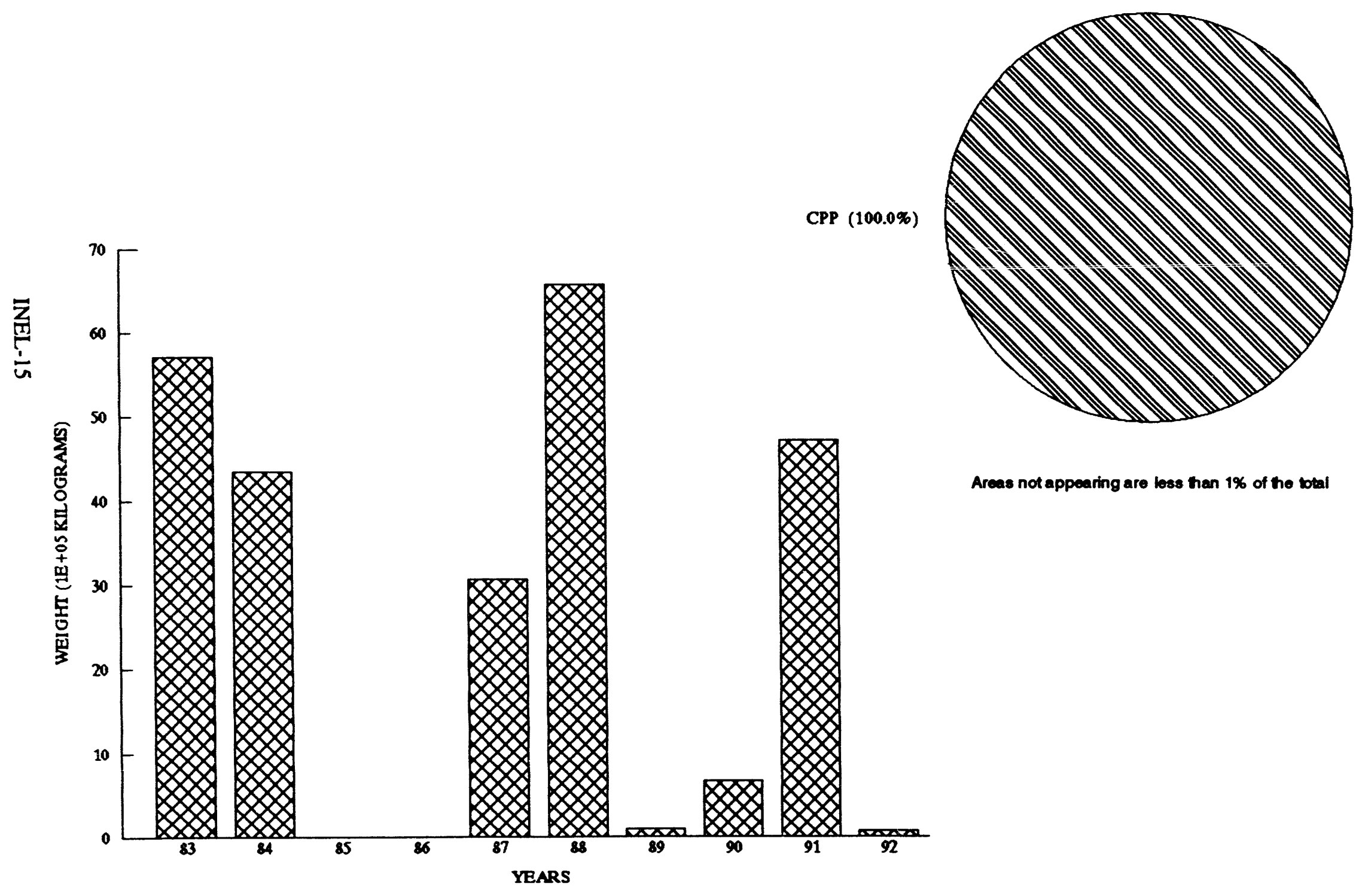



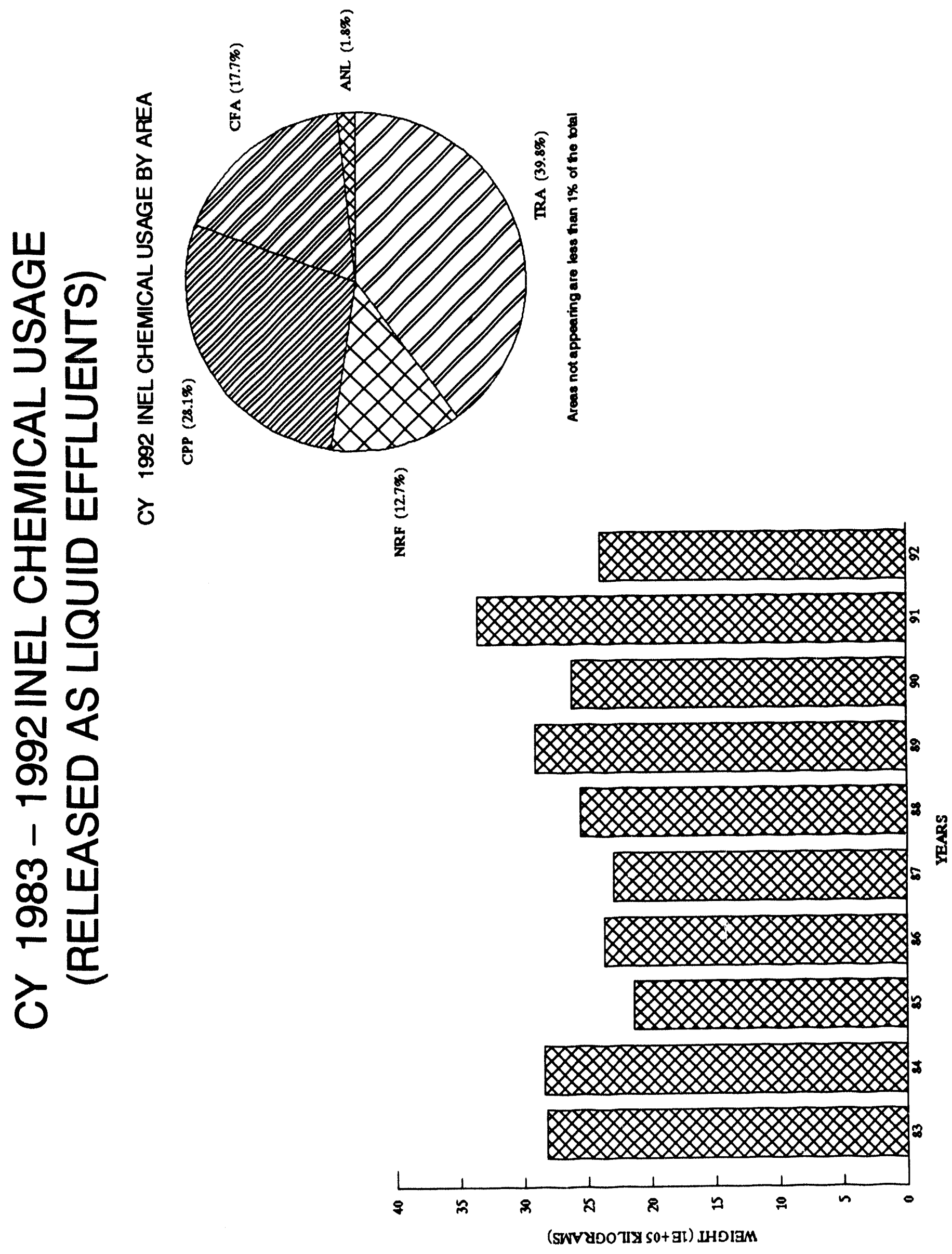

INEL-16 
RECORD TO DATE

\begin{tabular}{|c|c|c|c|c|c|c|c|c|c|c|c|c|}
\hline \multirow[b]{2}{*}{ AREA } & \multicolumn{4}{|c|}{$1971-1990$} & \multicolumn{4}{|c|}{1991} & \multicolumn{4}{|c|}{1992} \\
\hline & $\begin{array}{l}\text { FUEL OIL } \\
\text { (LITERS) }\end{array}$ & $\begin{array}{l}\text { SO2 } \\
\text { (KG) }\end{array}$ & $\begin{array}{l}\text { PART. } \\
\text { (KG) }\end{array}$ & $\begin{array}{l}\text { DIESEL } \\
\text { (LIITERS) }\end{array}$ & $\begin{array}{l}\text { FUEL OIL } \\
\text { (LITERS) }\end{array}$ & $\begin{array}{l}502 \\
\text { (KG) }\end{array}$ & $\begin{array}{l}\text { PART. } \\
\text { (KG) }\end{array}$ & $\begin{array}{l}\text { DIESEL } \\
\text { (LITERS) }\end{array}$ & $\begin{array}{l}\text { FUEL OIL } \\
\text { (LITERS) }\end{array}$ & $\begin{array}{l}\text { S02 } \\
\text { (KG) }\end{array}$ & $\begin{array}{l}\text { PART. } \\
\text { (KG) }\end{array}$ & $\begin{array}{l}\text { DIESEL } \\
\text { (LITERS) }\end{array}$ \\
\hline ANL & $34,711,724$ & 263,766 & 37,352 & & $1,434,803$ & 7,618 & 1,891 & & $1,882,426$ & 9,995 & 2,481 & \\
\hline ARA & $1,878,406$ & 13,212 & 523 & 56,119 & & & & & & & & \\
\hline CFA & $26,570,510$ & 259,908 & 22,262 & $1,731,934$ & 357,679 & 3,050 & 0 & 150,458 & 402,229 & 3,221 & & 137,084 \\
\hline CPP & $93,183,420$ & $1,263,627$ & 5,504 & & $5,575,326$ & 92,754 & 0 & & 713,476 & 11,871 & & \\
\hline CTF & 596,401 & 3,748 & 0 & 53,700 & 23,704 & 368 & 0 & 33,887 & & & & \\
\hline LOF & $1,508,115$ & 10,490 & 3 & 270,690 & & & & & & & & \\
\hline MHD & $2,596,712$ & 17,708 & 0 & & 21,641 & 130 & 0 & & 10,826 & 65 & & \\
\hline Z NRF & $89,973,904$ & $2,411,501$ & 155,127 & & $2,617,271$ & 24,152 & 0 & & $2,360,965$ & 35,275 & & \\
\hline $5^{\mathrm{PBF}}$ & $2,609,076$ & 16,855 & 231 & 526,602 & 91,927 & 627 & 0 & 18,757 & 64,113 & 364 & & 182 \\
\hline $\sin C$ & & & & & & & & & $1,113,536$ & 6,146 & & \\
\hline TAN & $39,629,913$ & 861,640 & 36,306 & 155,709 & & & & & & & & \\
\hline TRA & $47,513,788$ & $1,436,971$ & 182,189 & $32,388,537 \star$ & & 9,817 & 0 & $1,422,239 \star$ & & 9.627 & & $1,394,681^{\star}$ \\
\hline TSF & $7,505,074$ & 159,653 & 0 & 599,305 & $1,387,803$ & 10,989 & 0 & 453,336 & $1,372,438$ & 7,773 & & \\
\hline MMC & 443,055 & 2,820 & 0 & 28,493 & & & & & & & & \\
\hline URF & $4,143,223$ & 23,848 & 0 & 55,475 & 214,083 & 1,216 & 0 & & 134,215 & 525 & & \\
\hline Total & $352,863,321$ & $6,745,747$ & 439,498 & $35,866,563$ & $11,724,238$ & 150,721 & 1,891 & $2,078,677$ & $8,054,226$ & 84,861 & 2,481 & $1,531,948$ \\
\hline
\end{tabular}

- tra emergency generator 
SCHED. NO. INRPT031

INEL FUEL OIL \& DIESEL SLMWARY

RECORD TO DATE (CONTIMUED)

\begin{tabular}{|c|c|c|c|c|}
\hline \multirow[b]{2}{*}{ AREA } & \multicolumn{4}{|c|}{ TOTALS } \\
\hline & $\begin{array}{l}\text { FUEL OIL } \\
\text { (LITERS) }\end{array}$ & $\begin{array}{l}502 \\
\text { (KG) }\end{array}$ & $\begin{array}{l}\text { PART. } \\
\text { (KG) }\end{array}$ & $\begin{array}{l}\text { DIESEL } \\
\text { (LITERS) }\end{array}$ \\
\hline ANL & $38,028,954$ & 281,378 & 41,725 & \\
\hline ARA & $1,878,406$ & 13,212 & 523 & 56,119 \\
\hline CFA & $27,330,418$ & 266,179 & 22,262 & $2,019,477$ \\
\hline CPP & $99,472,223$ & $1,368,252$ & 5,504 & \\
\hline CTF & 620,105 & 4,116 & 0 & 87,587 \\
\hline LOF & $1,508,115$ & 10,490 & 3 & 270,690 \\
\hline MHD & $2,629,180$ & 17,903 & 0 & \\
\hline NRF & $94,952,141$ & $2,470,928$ & 155,127 & \\
\hline PBF & $2,765,116$ & 17,846 & 231 & 545,540 \\
\hline $\sin$ & $1,113,536$ & 6,146 & & \\
\hline TAN & $39,629,913$ & 861,640 & 36,306 & 155,709 \\
\hline TRA & $47,513,788$ & $1,456,415$ & 182,189 & $35,205,458$ \\
\hline TSF & $10,265,316$ & 178,414 & 0 & $1,052,640$ \\
\hline WMC & 443,055 & 2,820 & 0 & 28,493 \\
\hline WRF & $4,491,521$ & 25,590 & 0 & 55,475 \\
\hline & $372,641,785$ & $6,981,329$ & 443,870 & $39,477,187$ \\
\hline
\end{tabular}




\section{CY $1983-1992$ INEL FUEL OIL \& DIESEL USAGE}

CY 1992 INEL FUEL OIL \& DIESEL USAGE BY AREA
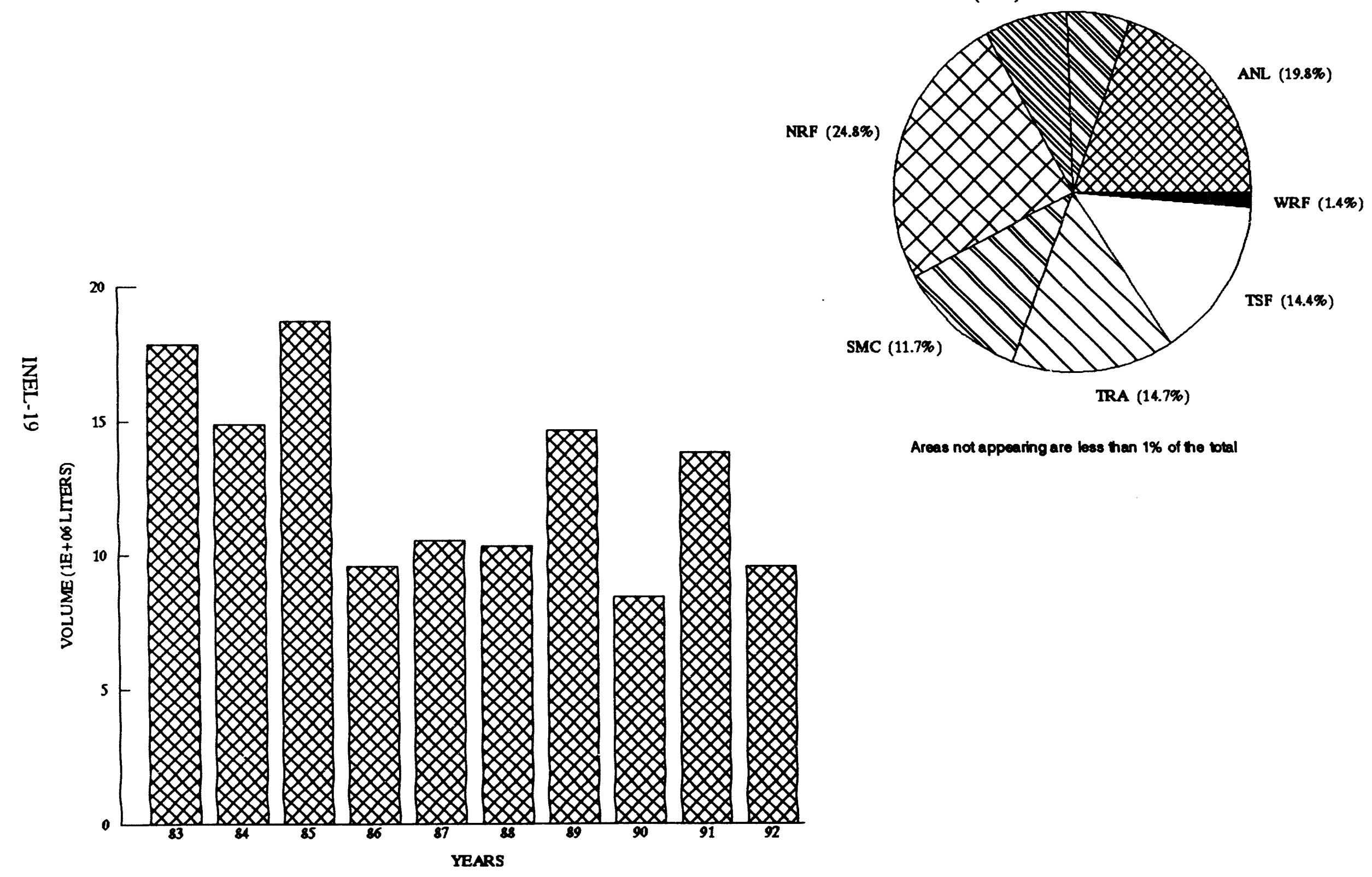

Areas not appearing are less than $1 \%$ of the total 


\section{CY 1983 - 1992 SULFUR DIOXIDE EMITTED INEL FUEL OIL \& DIESEL USAGE \\ CY 1992 SULFUR DIOXIDE EMITIED BY AREA FROM INEL FUEL O $\|_{P P} \&$ (1. DIESE) SEL USAGE}
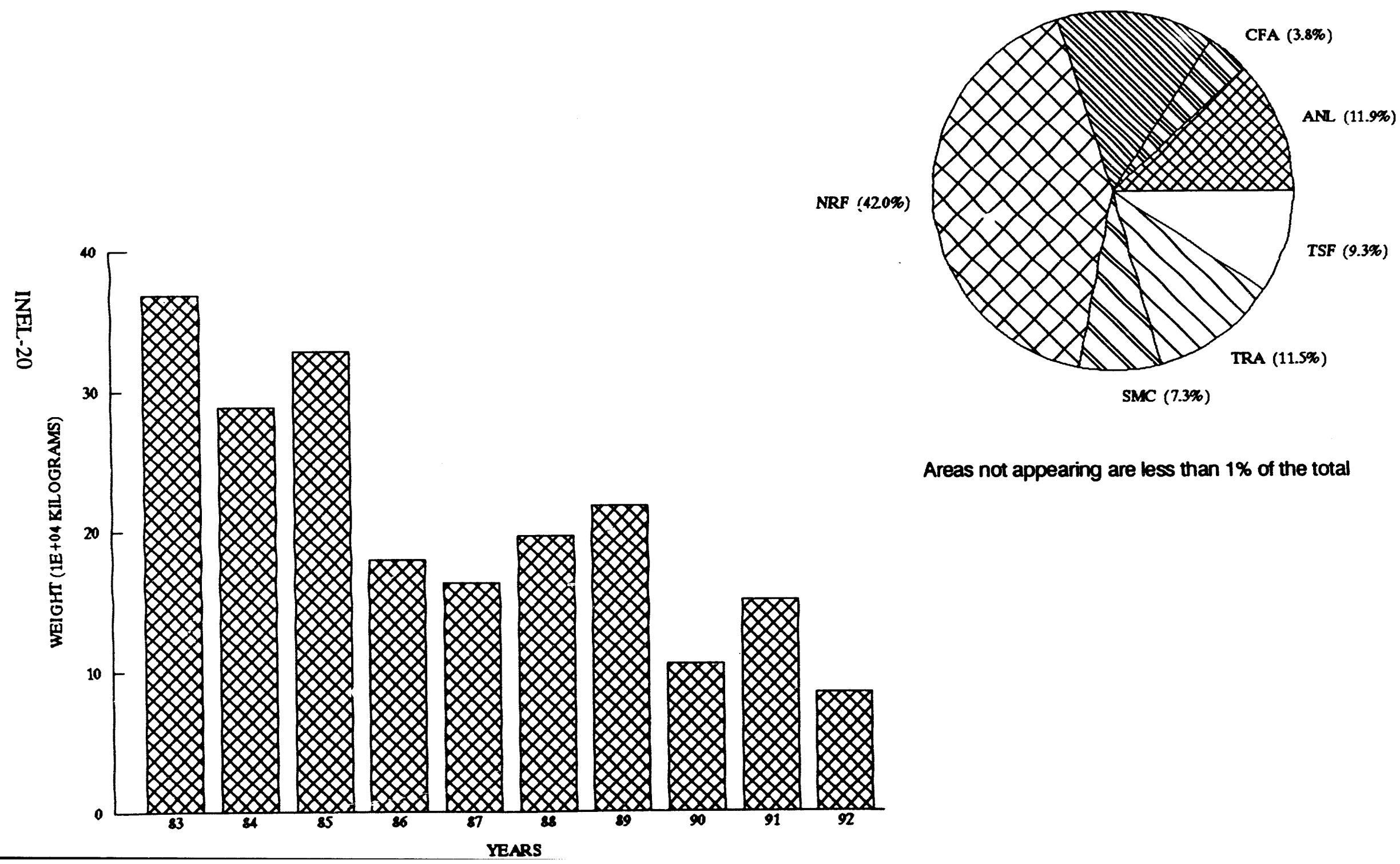

Areas not appearing are less than $1 \%$ of the total 


\section{CY 1983 - 1992 PARTICULATE EMITTED FROM INEL FUEL OIL \& DIESEL USAGE}

CY 1992 PARTICULATE EMITTED BY AREA

FROM INEL FUEL OIL \& DIESEL USAGE

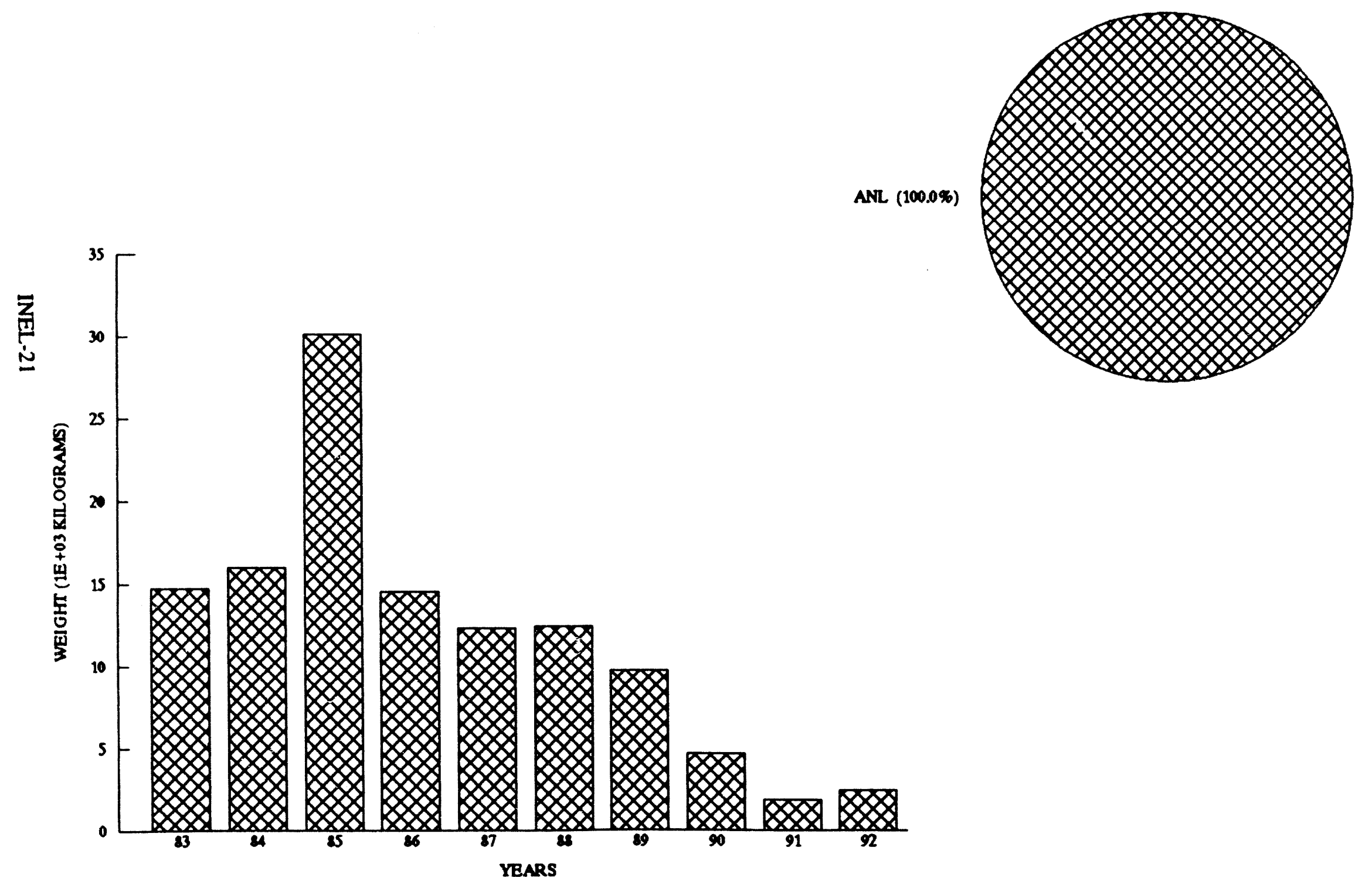


SCMED. NO IMRPTO32A

INEL MATER USAGE SUMMARY IN THOUSAMD LITERS RECORD TO DATE

\begin{tabular}{|c|c|c|c|c|c|}
\hline AREA & $1971-1989$ & 1990 & 1991 & 1992 & TOTAL \\
\hline ANL & $8,293,393$ & 546,145 & 388,564 & 388,760 & $9,616,870$ \\
\hline ARA & $1,451,289$ & & & & $1,451,289$ \\
\hline CFA & $4,506,486$ & 342,841 & 331,431 & 377,274 & $5,558,033$ \\
\hline CPP & $32,301,852$ & $2,398,819$ & $2,146,863$ & $2,502,782$ & $39,350,316$ \\
\hline cTF & 238,689 & 96,028 & 456,490 & 2,953 & 794,160 \\
\hline LOF & $2,655,483$ & & & & $2,655,483$ \\
\hline MHD & 312,635 & 21,773 & 25,394 & 700 & 360,502 \\
\hline MRF & $26,691,823$ & 872,708 & 880,799 & $1,112,774$ & $29,558,104$ \\
\hline PBF & 621,063 & 21,949 & 30,624 & 15,227 & 688,864 \\
\hline TAM & $3,581,392$ & & & & $3,581,392$ \\
\hline TRA & $56,022,029$ & $2,576,814$ & $2,374,097$ & $2,255,909$ & $63,228,849$ \\
\hline TSF & 379,503 & 96,235 & 98,540 & 148,431 & 722,709 \\
\hline Inc & 386,917 & 999 & 1,567 & 1,814 & 391,298 \\
\hline WRF & 597,822 & 10,097 & 10,180 & 10,455 & 628,553 \\
\hline TOTAL & $138,040,376$ & $6,984,408$ & $6,744,550$ & $6,817,087$ & $158,586,421$ \\
\hline
\end{tabular}


SCHED. NO INRPTO32

INEL WATER PUAPED SUMARY IN THOUSAND LITERS

RECORD TO DATE

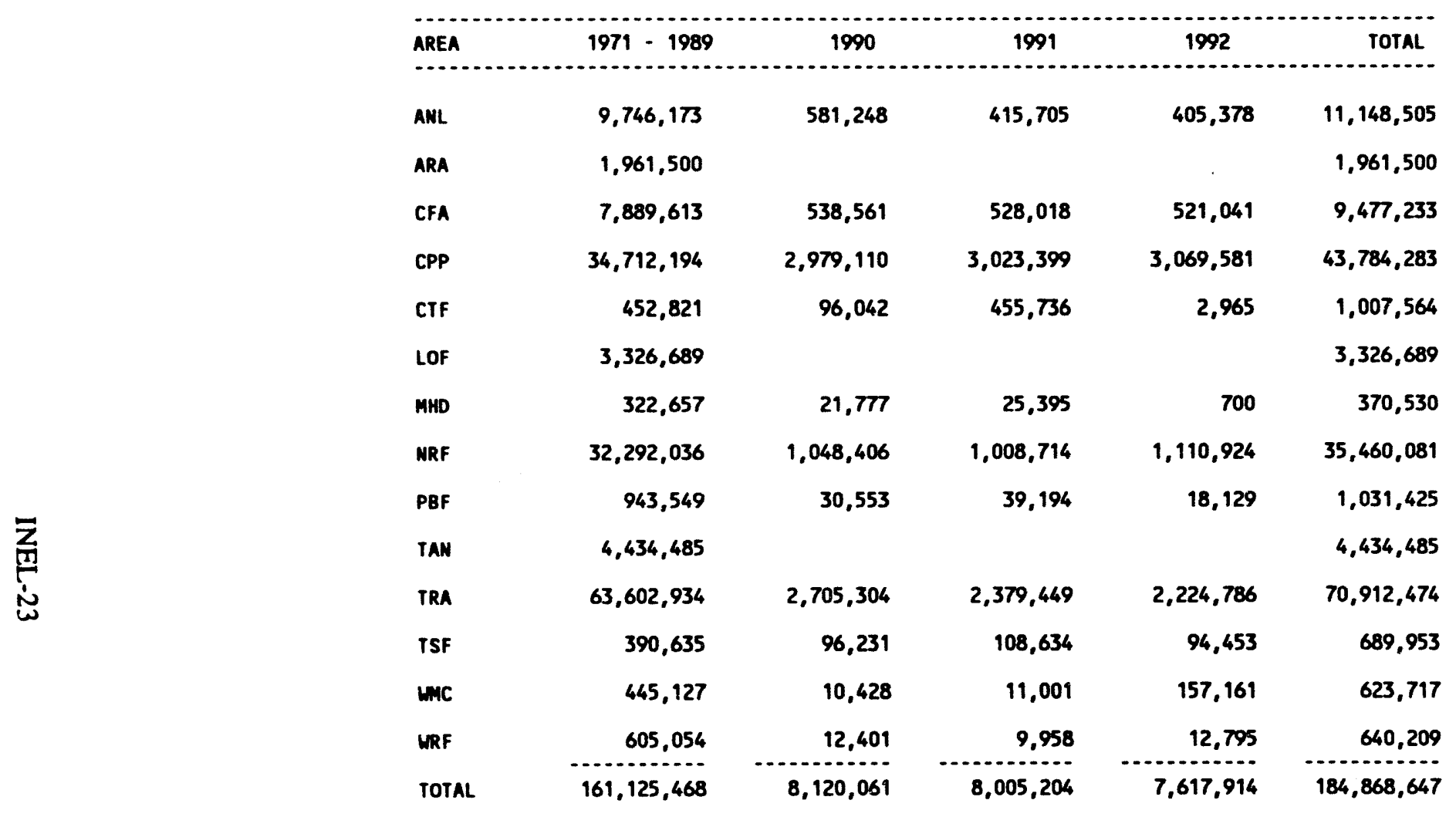




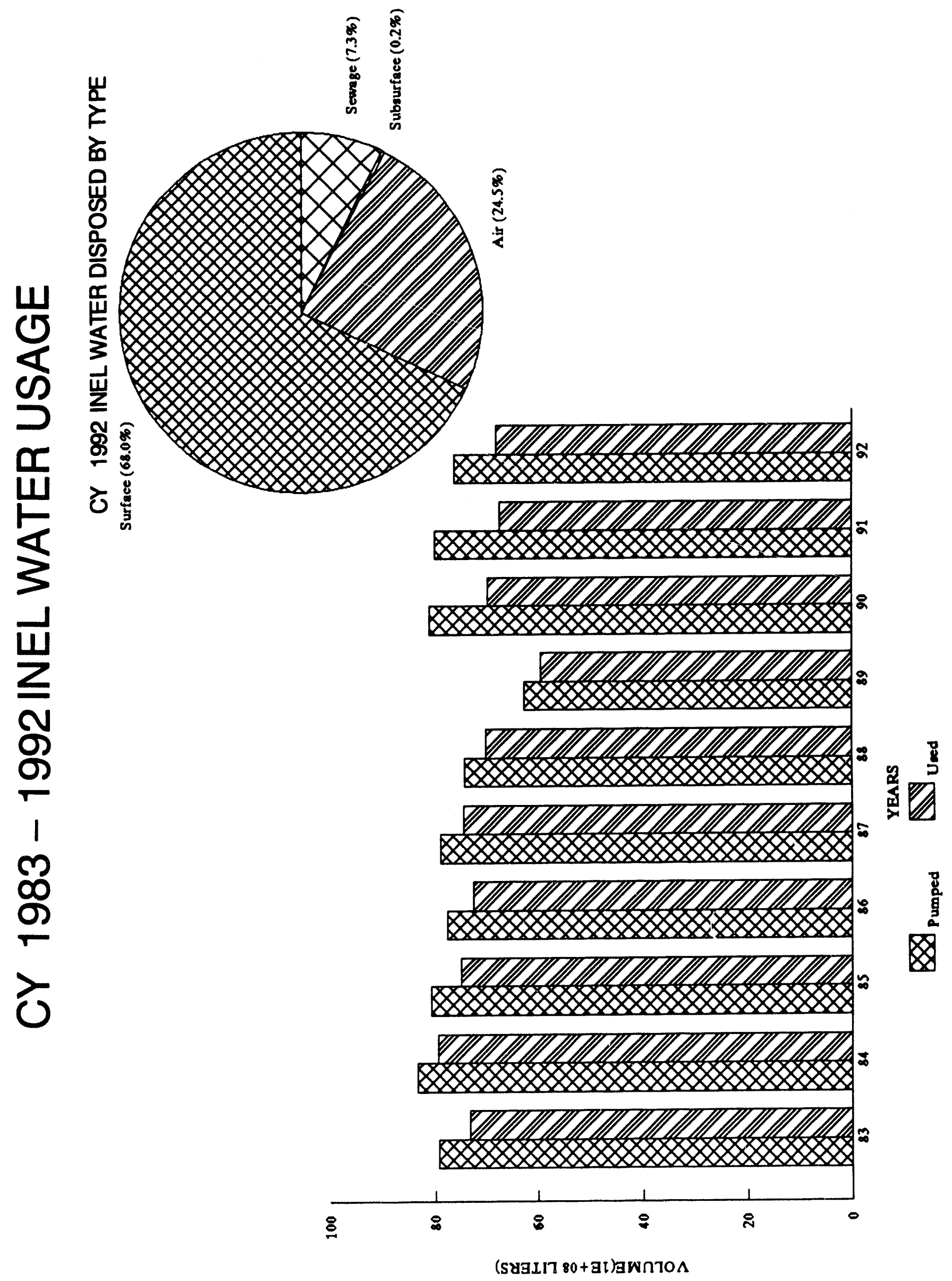

INEL-24 


\section{CY 1992 INEL WATER USAGE}

CY 1992 INEL WATER PUMPED BY AREA

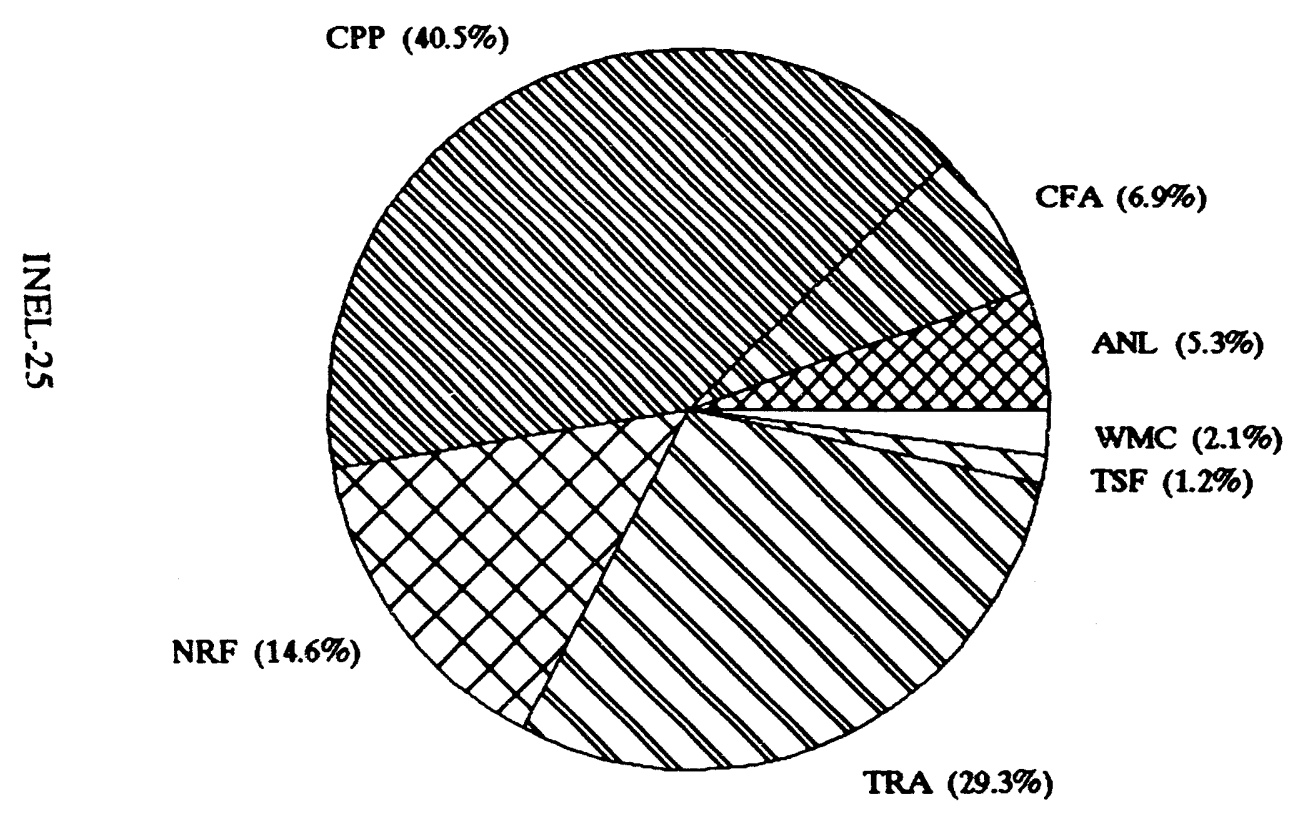

Areas not appearing are less than $1 \%$ of the total
CY 1992 INEL WATER DISPOSED BY AREA

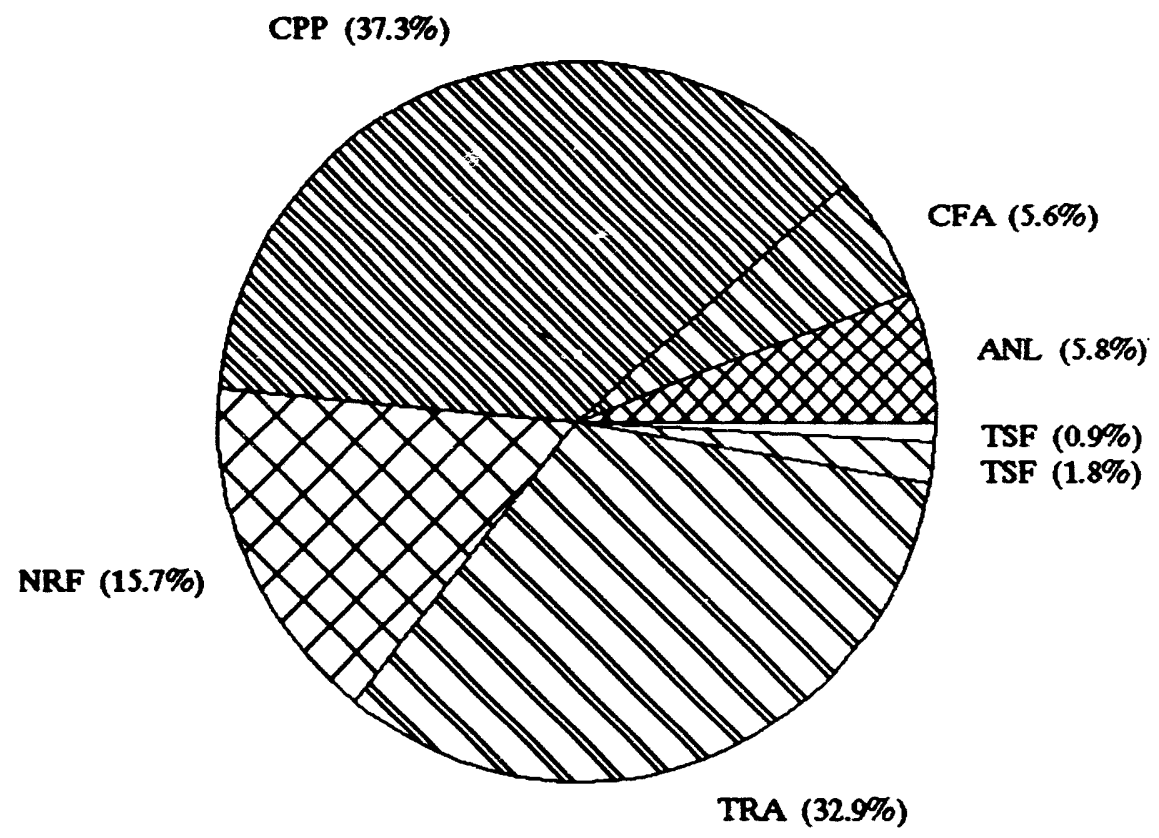

Areas not appearing are less than $1 \%$ of the botal 
RECORD TO DATE

\begin{tabular}{|c|c|c|c|c|c|c|c|c|c|c|c|c|}
\hline \multirow[b]{2}{*}{ Area } & \multicolumn{3}{|c|}{$1971-1989$} & \multicolumn{3}{|c|}{1990} & \multicolumn{3}{|c|}{1991} & \multicolumn{3}{|c|}{1992} \\
\hline & $\begin{array}{l}\text { Tons } \\
\text { Burned }\end{array}$ & 502 (mg) & NOX (mg) & $\begin{array}{l}\text { Tons } \\
\text { Burned }\end{array}$ & $\mathrm{sO2}$ (mg) & NOX (mg) & $\begin{array}{c}\text { Tons } \\
\text { Burned }\end{array}$ & $\mathrm{sO2}$ (mg) & NOX (mg) & $\begin{array}{c}\text { Tons } \\
\text { Burned }\end{array}$ & SO2 (mg) & NOX (mg) \\
\hline CPP & 39,960 & 396 & 215 & 12,091 & 123 & 43 & 3,291 & 37 & 143 & 12,464 & 17 & 64 \\
\hline TOTAL & 39,960 & 396 & 215 & 12,091 & 123 & 43 & 3,291 & 37 & 143 & 12,464 & 17 & 64 \\
\hline
\end{tabular}


SCHED. NO. INRPT033

\section{INEL COAL SUMMARY}

RECORD TO DATE (CONTINUED)
TOTALS

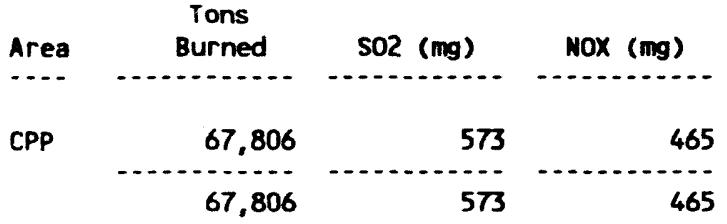

ב⿱彐

DETAILS MAY NOT ADD UP TO TOTALS BECAUSE OF ROUNDING $O$ IN A COLUMN INDICATES A TOTAL OF $<0.5$ 


\section{CY 1987 - 1992 INEL COAL USAGE}

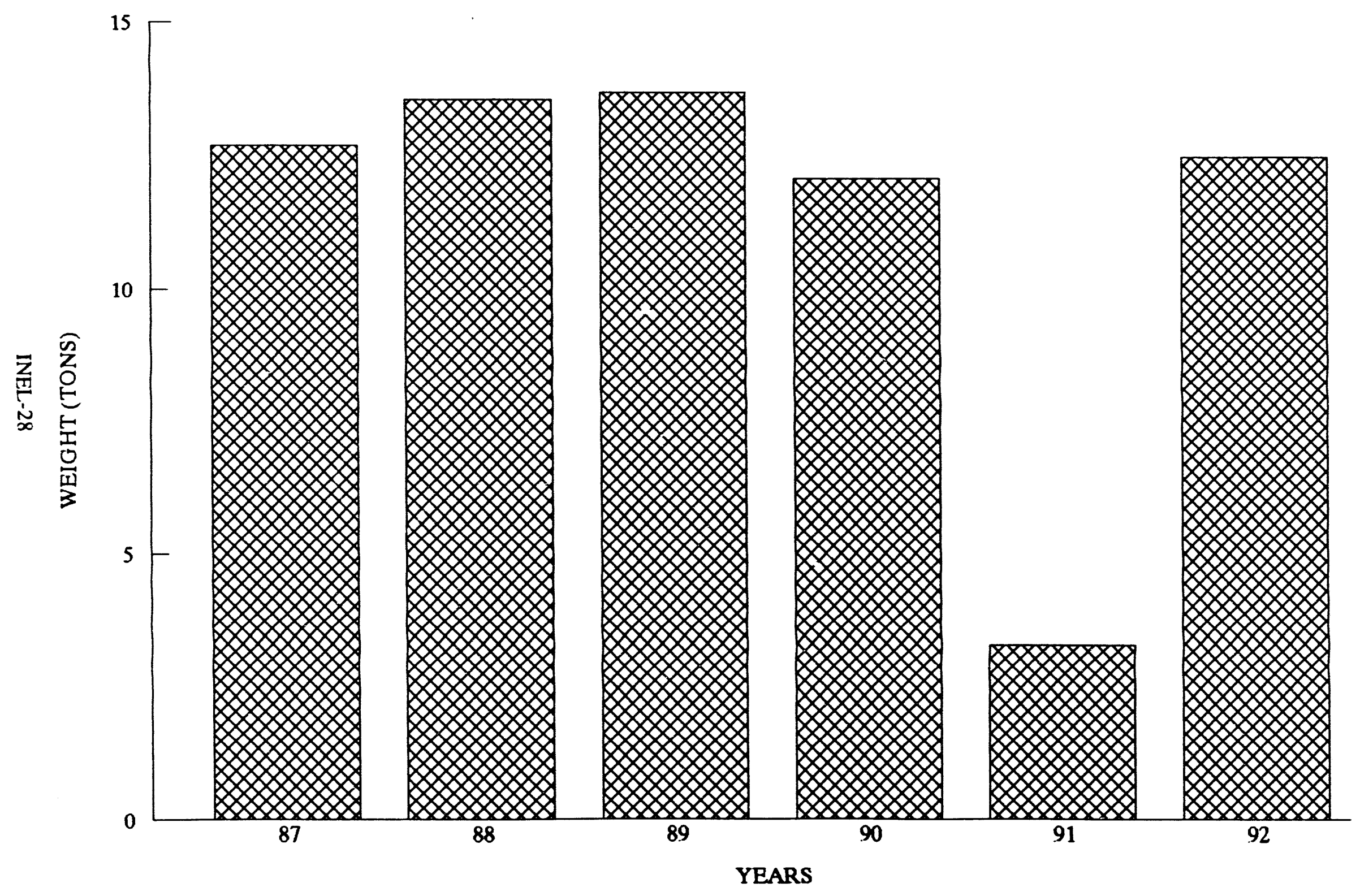




\section{CY 1987 - 1992 SULFUR DIOXIDE EMITTED FROM INEL COAL USAGE}

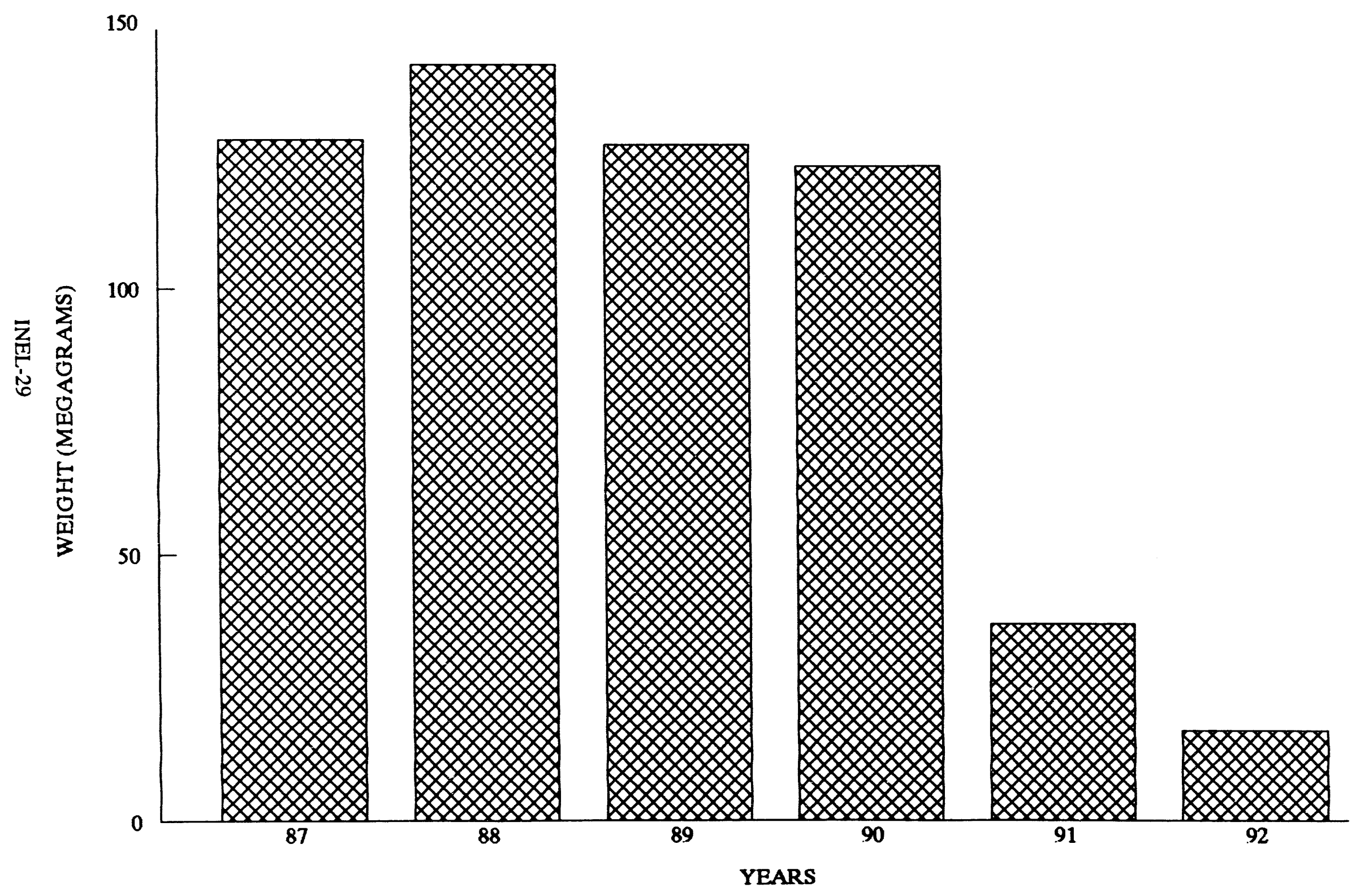


$0 \varepsilon-7 \exists N I$

WEIGHT (MEGAGRAMS)
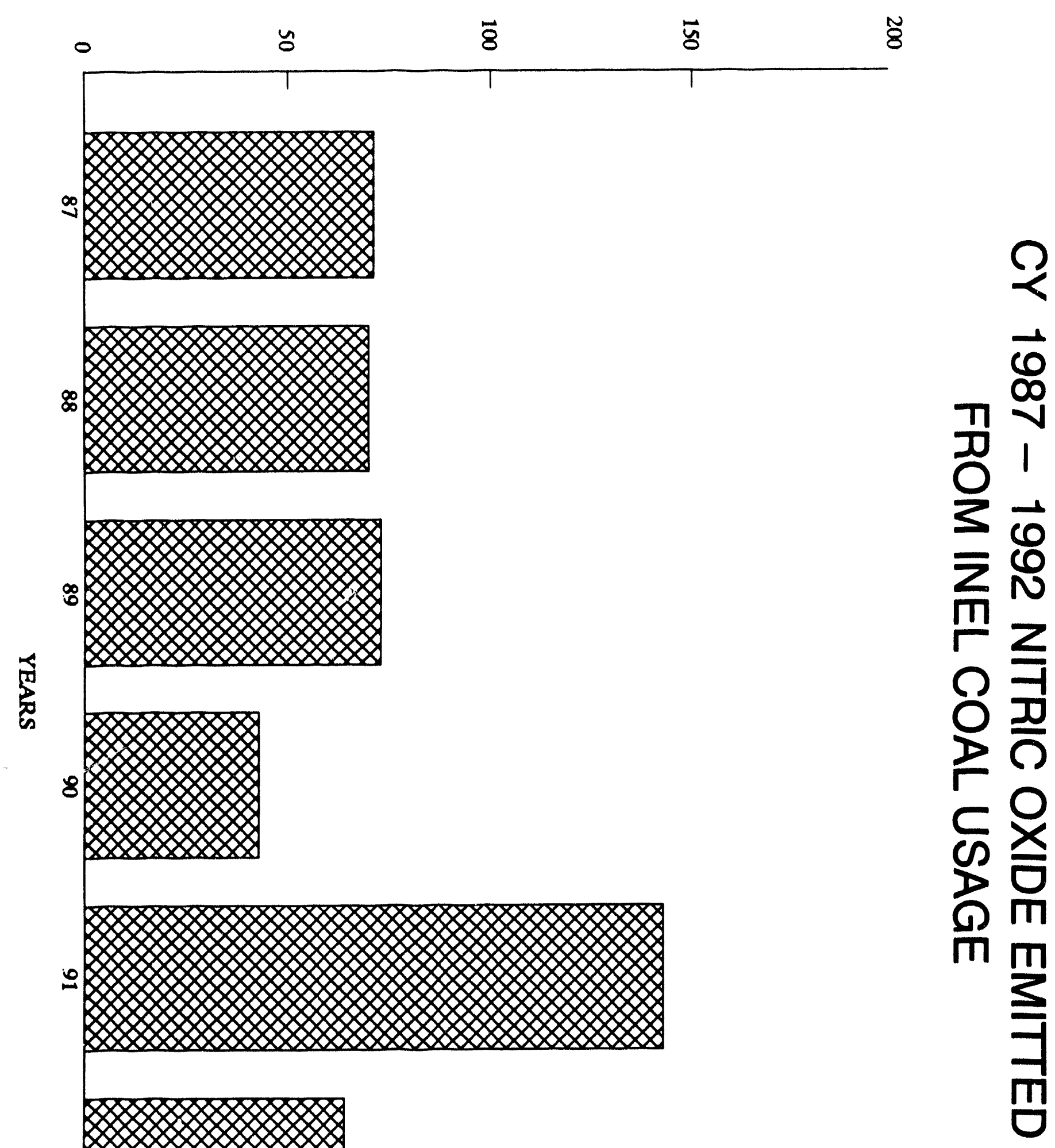
SCHED. NO. IMRPTO34

INEL MASTE OIL SUMMARY IN LITERS RECORD TO DATE

\begin{tabular}{|c|c|c|c|c|c|c|c|c|c|c|c|c|}
\hline AREA & $1971-1982$ & 1983 & 1984 & 1985 & 1986 & 1987 & 1988 & 1989 & 1990 & 1991 & 1992 & TOTAL \\
\hline ANL & 22,915 & 2,536 & 2,290 & 2,425 & 1,230 & 1,052 & 3,218 & 2,173 & & & 2,271 & 40,111 \\
\hline ARA & & & & & & 208 & 341 & & & 1,344 & & 1,893 \\
\hline BRX & & & & & & & & & & 454 & & 454 \\
\hline CFA & & & & & 27,028 & 40,598 & 74,818 & 35,412 & 30,586 & 50,660 & 25,165 & 284,268 \\
\hline CPP & & & & & 3,785 & 3,785 & & & 6,871 & & 3,028 & 17.470 \\
\hline IRC & & & & & & 416 & & & & 927 & 189 & 1,533 \\
\hline MRF & 331.529 & 37,475 & 49,256 & 10,115 & 50,403 & 70,976 & 77.650 & 129,499 & 38,157 & 25,268 & 11,545 & 831,872 \\
\hline PBF & 4.997 & & & & & 492 & 416 & 208 & & 17,091 & 189 & 23,394 \\
\hline PTI & & & & & & & & 208 & & 1,136 & & 1,344 \\
\hline TAN & & & & & 3,123 & 5,072 & 13,325 & 1,420 & 17,413 & 14,195 & 757 & 55,305 \\
\hline TRA & 5,678 & & & & 4,921 & 7,238 & 3,312 & 12,870 & 16,448 & 6,057 & 10,221 & 66,744 \\
\hline WMC & & & & & 757 & 1.666 & 833 & 1,136 & 625 & 208 & & 5,224 \\
\hline WRF & & & & & & 208 & 1,079 & 341 & 1,325 & 4,164 & & 7,117 \\
\hline & 365,119 & 40,012 & 51,546 & 12,540 & 91,267 & 131,713 & 174,991 & 183,266 & 111,423 & 121,504 & 53,367 & $1,336,728$ \\
\hline
\end{tabular}




\section{INEL 1992 YEAR-TO-DATE SUMMARIES}

INEL Landfill Disposed Solid Waste Summary In Cubic Meters For January

Through December, 1992

INEL Landfill Disposed Solid Waste Summary, Fourth Quarter and Year-To-Date, 1992

Airborne And Liquid Industrial Waste Substances Summary In Kilograms For January Through December, 1992

INEL Year-To-Date Water Usage Summary For January Through

December 1992 INEL-41

INEL Quarterly Fuel Oil and Diesel Summary For January Through

December 1992 INEL-42

INEL Waste Oil Summary In Gallons January Through December 1992 INEL-43

INEL Year-To-Date Coal Usage Summary for January Through

December 1992 INEL-44

Summary INEL Hazardous Waste Generated For January-December 1992 INEL-45

Summary INEL Mixed Waste Generated For January-December 1992 INEL-47 
SCHED. NO. INRPTO38

IMEL LANDFILL DISPOSED SOLID HASTE SUMMARY IN CUBIC METERS

FOR JANUARY THROUGH DECEMBER, 1992

RPT 3-1

CFA CPP
NRF PBF TAN TRA IMA TOTALS

ASBESTOS

ASPHALT

CAFETERIA GARBAGE

DIRT AMD GRAVEL

MASONRY AND CONCRETE

SCRAP METAL

TRASH, SWEEPINGS, ETC.

MEEDS, GRASS AND TREES

率

WOCO AND SCRAP LUMBER

OTHER

TOTALS

\begin{tabular}{|c|c|c|c|c|c|c|c|c|c|}
\hline 291 & 25 & 86 & & 207 & 3 & 28 & 43 & 1 & 683 \\
\hline 67 & 83 & 1,295 & & 95 & 2 & 47 & 8 & & 1,597 \\
\hline 560 & 1,819 & 26 & & 1,344 & 12 & 12 & & & 3,773 \\
\hline 25 & 781 & 488 & & 729 & 1 & 170 & 3 & & 2,197 \\
\hline 232 & 101 & 1,136 & & 72 & 3 & 36 & 8 & 1 & 1,589 \\
\hline 206 & 263 & 370 & & 257 & 25 & 221 & 66 & 27 & 1,434 \\
\hline \multirow[t]{2}{*}{5,929} & 6,060 & 7,957 & 147 & 10,014 & 638 & 2,816 & 2,821 & 695 & 37,076 \\
\hline & 196 & 268 & & 31 & 13 & 23 & 47 & & 576 \\
\hline 437 & 286 & 987 & & 365 & 36 & 247 & 197 & 28 & 2,582 \\
\hline 10 & 33 & 50 & & 36 & 0 & 11 & 6 & 3 & 150 \\
\hline 7,756 & 9,645 & 12,663 & 147 & 13,150 & 734 & 3,611 & 3,199 & 754 & 51,658 \\
\hline
\end{tabular}


PAGE: 1

SCHED. MO. INRPTOO3
IDAHO OPERATIONS OFFICE

UNITED STATES DEPARTMENT OF ENERGY

INEL NONRADIOLOGICAL WASTE MANAGEMENT INFORMATION SYSTEM

INEL LANDFILL DISPOSED SOLID WASTE SUMMARY

FOURTH QUARTER AND YEAR-TO-DATE 1992

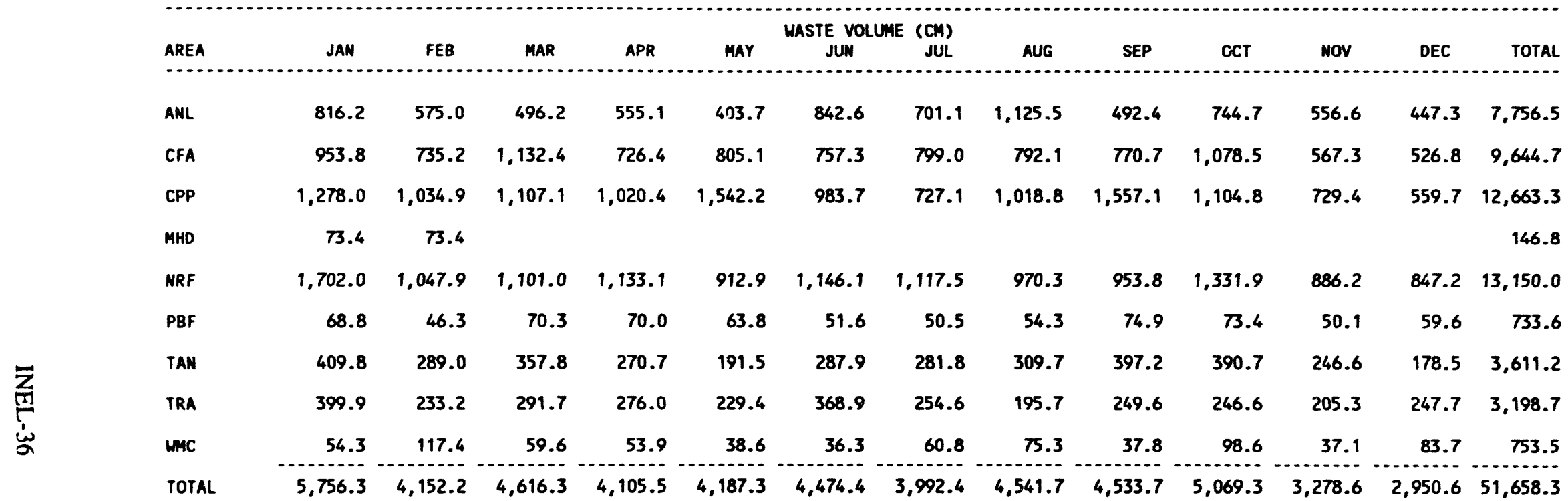


FOR IANUARY TMROUGH DECEMBER, 1992

Substance

CFA

CPP

CTF

MRF

PBF

PUT

TRA

TSF

MRF

(1- HYDROXYETHYL IDENE)BI SPHOSPOWIC

1-BROMO-3-CHLORO-5, 5-DIMETHYLHYDANT

1-BROMO-3-CHLORO-5,5-DIMETHYL HYDAMTOIN

ACRYLIC POL YMER

ALKYL DIMETHYL BENZYL AMONIUN CHLORIDE

ALUMINUN NITRATE

AMINEMETHYLPRCPAMOL

AROMATIC SOLVENTS

ARSENIC

B-BROMO-B-NITROSTYRENE

BARIUN

BARIUA SULFATE

BENZOTRIAZOLE

BETZ 20

BIS( TRICHLOROMETHYL) SUL FONE

CaOHIOH

CALCIUN CHLORIDE

三 CALCIUM FLUORIDE

calciuar jon

CALCIUN NITRATE

CALCIUN PERCHLORATE

CALCIUM PHOSPHATE, TRIBASIC

CALCIUM SULFATE, AMHYOROUS

CARBOXYALKYLATED POLYSACCHARIDE

CARBOXYLATED LIGNIM

CHLORIDE ION

CYCLOHEXYLAMIME

DIMETHYLI SOPROPAMOL AMINE

DCOECYLGUANIDINE HYDROCHLORIDE

DREMGARO

ETHYL ALCOHOL

ETHYLAMINE

ETHYLENEDIAMINE TETRAMCETIC ACID

FLUORIDE ION

HEAVY AROMATIC MAPTHA

HYDROGEM CHLORIDE

HYPOCHLORITE IOM

IMPURITY

ISOPROPANOL

I SOPROPYL ALCOHOI

JANITORIAL SUPPLIES

LAUMDRY PRODUCTS

METHYLENE BIS(THIOCYAMATE)

MORPHOLINE
953

1,479

362

362
., 434
785

168

30

175

4,936

$\begin{array}{lll}16,872 & 545,324 & 154,567\end{array}$

47
$2 \quad 46$
44

482

19

26

291

5,710

24

111

157

2,608

18

157

8

DETAIL MAY MOT ADO UP TO TOTALS BECAUSE OF ROUNDING

O IN A COLUM IMOICATES A TOTAL OF $<0.5$ 
(1- HYDROXYETHYLIDENE)BISPHOSPONIC

1-BROMO-3-CHLORO-5,5-DIMETHYLHYDANT

1-BROM-3-CHLORO-5, 5-DIMETHYL HYDAMTOIN

ACRYLIC POLYMER

ALKYL DIMETHYL BENZYL AMHONIUM CHLORIDE

ALUMINUM NITRATE

MMIMEMETHYLPROPANOL

AROMATIC SOLVENTS

ARSENIC

B-BRONO-B-NITROSTYREME

BARIUIN

BARIUN SULFATE

BENZOTRIAZOLE

BET2 20K

BIS(TRI CHL OROMETHYL) SUL FONE

CaDMIITH

CALCIUA CHLORIDE

CALCIUM FLUORIDE

$\overline{\text { CALCIUN ION }}$

(3) CALCIUM NITRATE

i CALCIUM PHOSPHATE, TRIBASIC

CALCIUM SULFATE, ANHYDROUS

CARBOXYALKYLATED POLYSACCHARIDE

CARBOXYLATED LIGNIN

CHLORIDE ION

CHROMIUN

CYCLOHEXYLAMINE

DIMETHYLI SOPROPANOL AMINE

DCDECYLGUANIDINE HYDROCHLORIDE

DREWGARD

ETHYL ALCOHOL

ETHYLAMINE

ETHYLENEDIAMINE TETRAMCETIC ACID

FLUORIDE ION

HEAVY AROMATIC MAPTHA

HYDROGEN CHLORIDE

HYPOCHLORITE IOW

IMPURITY

ISOPROPANOL

ISOPROPYL ALCOHOL

JANITORIAL SUPPLIES

JANITORIAL SUPPLIES

METHYLEE BIS(THIOCYANATE)

MORPHOLINE

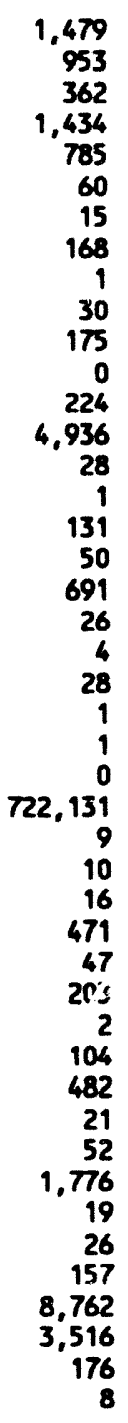

DETAIL maY MOT ADD UP TO TOTALS BECAUSE OF ROUNDIMG

O IN A COLUMN INDICATES a TOTAL OF $<0.5$ 
FOR JAMUARY THROUGH DECEMBER, 1992

Substance

ANL

CFA CPP CTF NRF PBF PUT TRA TSF WRF

N-METHYLPYRROLIDONE

NITRATE ION

NITRIC OXIDE

NITROGEN DIOXIDE

MONYLPHENOXYPOL Y(ETHYLENEOXY)ETHANOL

MONYLPHENOXYPOLY

PHOSPHATE ION
PHOTO LAB CHEMICAL

POLYACRYLATE

POLYALKYLENE GLYCOL

POTASSIUM HYDROXIDE

SALT OF EDTA

SELENIUN

SILICONE EMULSION

SOOIUM HYDRATE SOLUTIOH

SOOIUA HYDROXIDE

SODIUN ION

SODIUM LIGNOSULFONATE

SOOIUM SULFATE

$\bar{z}$ SOOIUM SULFITE

(1) SCOIU⿴囗十⺝ TOLYTRIAZOLE

SOOIUW TRIPOLYPHOSPHATE

STOODARD SOLVENT

SULFATE ION

SULFITE ION

TETRAPOTASSIUM PYROPHOSPHATE

TOTAL HARDMESS

TRIBUTYLTIN OXIDE

Total $\pi$

39,847

847

$21 \quad 6,881$

792
282

136

858

960

282
0

44

2

8

4,085

$11,041 \quad 342,845$

1

4

1
36,434

83
.434
3

172

56,355

207,566

671
92,023

1

31

$92,345 \quad 3,361$

$\begin{array}{rr}461 & 311 \\ & 12\end{array}$

1,479

$\begin{array}{rr}1 & 267,095 \\ 3, .696 & 148,906\end{array}$

148,906 157

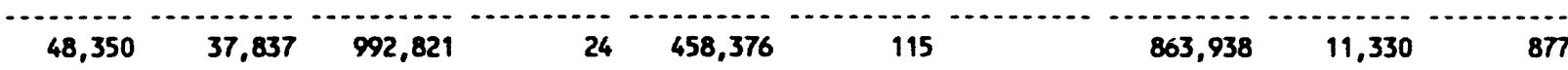




\section{N-METHYLPYRROLIDONE}

NITRATE ION

NITRIC OXIDE

NITROGEN DIOXIDE

NOWYLPHENOXYPOL Y(ETHYLENEOXY)ETHANOL

PHOSPHATE ION

PHOTO LAB CHEMICAL

POL YACRYLATE

POLYALKYLENE GLYCOL

POTASSIUN HYDROXIDE

SALT OF EDTA

SELENIUN

SILICONE EMULSION

SCOIUM HYDRATE SOLUTION

SODIUA HYOROXIDE

SOOIUA ION

SODIUN LIGNOSULFONATE

SODIUN SULFATE

$Z$ SODIUN SULFITE

S SOOIUM TRIPOLYPHOSPHATE

STCODARD SOLVENT

SULFATE ION

SULFATE ION

T5

SULFITE ION

TETRAPOTASSIUN PYROPHOSPHATE

TOTAL HARDNESS

TRIBUTYLTIN OXIDE

$\begin{array}{r}75 \\ 39,847 \\ 765 \\ 6,881 \\ 21 \\ 1,953 \\ 792 \\ 282 \\ 2,042 \\ 1,582 \\ 0 \\ 2 \\ 31 \\ 8 \\ 703 \\ 546,011 \\ 104 \\ 1 \\ 4 \\ 1,479 \\ 1 \\ 83 \\ 641,343 \\ 232 \\ 267,095 \\ 3,696 \\ 148,906 \\ 157 \\ \hline 2, .9 \\ \hline 213,668\end{array}$

Total

75

765

881
21

953

832

0

31

703

011

104

.479

83
343

232

$2,413,668$ 
PAGE: 1

SCHED. NO. INRPT002-A
IDAHO OPERATIONS OFFICE

INEL NONRADIOLOGICAL WASTE MANAGEMENT INFORMATION SYSTEM

INEL YEAR-TO-DATE WATER USAGE SUMMARY

FOR JANUARY THROUGH DECEMBER 1992

RPT 2-1

(ALL VOLUMES = MEAREST THOUSAND LITERS)

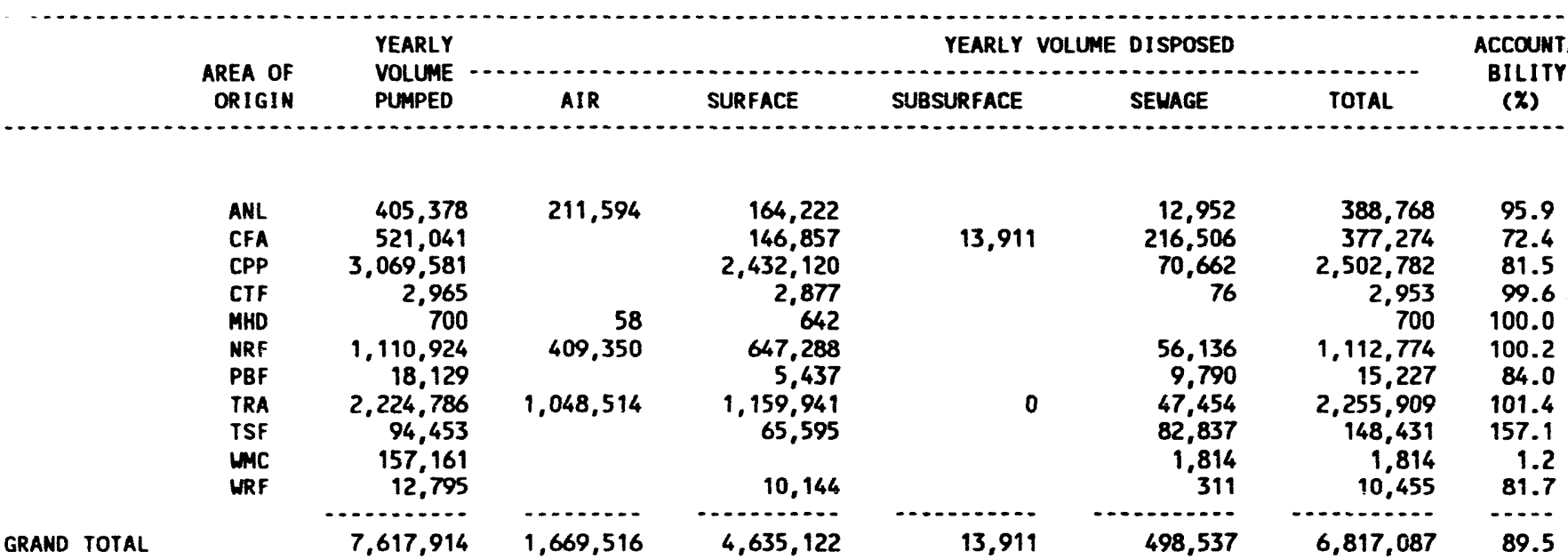


PAGE: 1

SCHED. NO. INRPTOO6

IOAHO OPERATIONS OFFICE

UNITED STATES DEPARTMEMT OF EMERGY

19- JUN-93

INEL NONRADIOLOGICAL MASTE MAMAGEMENT INFORMATION SYSTEM

INEL QUARTERLY FUEL OIL AND DIESEL SUMMARY

FOR JAMUARY THROUGH DECEMBER 1992

RPT 5-1

FUEL OIL IN LITERS

KILOGRAMS DISCHARGED

\begin{tabular}{|c|c|c|c|c|c|c|c|c|}
\hline \multirow[b]{2}{*}{$\begin{array}{c}\text { AREA } \\
\text { OF ORIGIN }\end{array}$} & \multicolumn{5}{|c|}{ FUEL OIL IN LITERS } & \multicolumn{3}{|c|}{ KILOGRAMS DISCHARGED } \\
\hline & TYPE 2 & TYPE 5 & TYPE 6 & KEROSENE & DIESEL & s02 & NOX & PARTICULATES \\
\hline ANL & $1,882,426$ & & & & & 9,995 & & 2,481 \\
\hline CFA & 402.229 & & & & 137,084 & 3,221 & & \\
\hline CPP & 713,476 & & & & & 11,871 & & \\
\hline MHD & 10,826 & & & & & 65 & & \\
\hline NRF & & $2,360,965$ & & & & 35,275 & & \\
\hline PBF & 64,113 & & & & 182 & 364 & & \\
\hline SMC & $1,113,536$ & & & & & 6,146 & & \\
\hline TRA & & & & & $1,394,681$ & 9.627 & & \\
\hline TSF & $1,372,438$ & & & & & 7,773 & & \\
\hline WRF & 134,215 & & & & & 525 & & \\
\hline GRAND TOTAL: & $5,693,261$ & $2,360,965$ & & & $1,531,948$ & 84,861 & & 2,481 \\
\hline
\end{tabular}


PAGE : 1

SCHED. NO. INRPT016
IDAHO OPERATIONS OFFICE

19- JUN-93

UNITED STATES DEPARTMEMT OF ENERG

INEL NONRADIOLOGICAL WASTE MAMAGEMENT INFORMATION SYSTEM

INEL WASTE OIL SUMARY IN GALLONS

JAMUARY THROUGH DECEMBER 1992
RPT 9-I

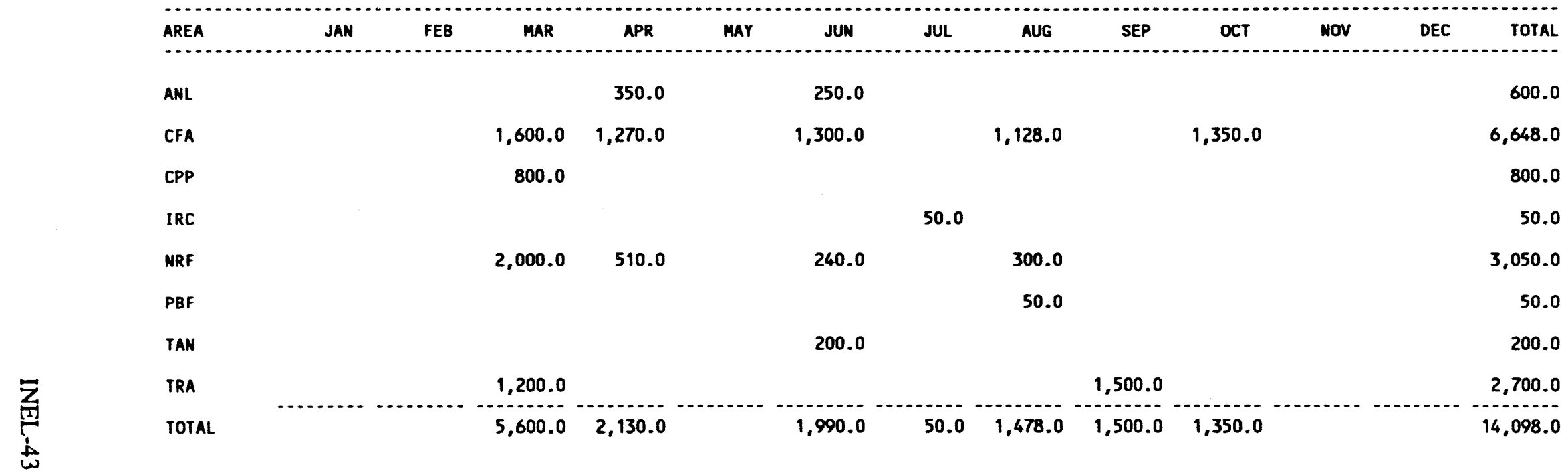


PAGE: 1

SCHED. NO. INRPT018

IDAHO OPERATIONS OFFICE

UNITED STATES DEPARTMENT OF ENERGY

RPT 5-I

IMEL YEAR-TO-DATE COAL USAGE SUMMUAY

FOR JANUARY THROUGH DECEMBER 1992

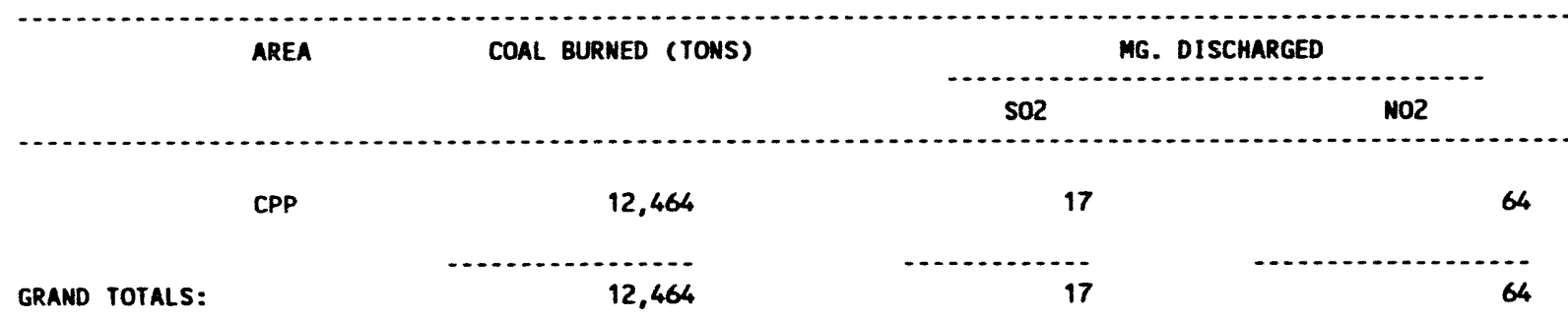


SUMWARY IMEL

FOR Janury - Decenter 1992

AREA

ANL

Gross Volumes: 4.16E-01

Gross Weights: $4.63 E+05$

CFA

Gross Volumes: $\quad 3.97 E-01$

Gross Volumes:

Gross Weights:

IET

Gross Volumes:

$\bar{Z}$ Gross Volumes: $\begin{aligned} & 2.43 E+00 \\ & \text { Gross Weights: }\end{aligned}$

茴 MRF

$\begin{array}{ll}\text { L Gross Volumes: } & 4.47 E+00 \\ \text { in Gross Weights: } & 3.81 E+06\end{array}$ PBF

Gross Volumes:

Gross Weights:

PII

Gross Volumes:

Gross Weights:

SLR

Gross Volumes:

Gross Weights:

TAN

Gross Volumes: 2.08E-01

Gross Weights: $1.36 E+05$

Gross Volumes: $7.57 \mathrm{TE}-02$

Gross Weights: $2.18 E+04$

TSB

Gross Volumes:

Gross Weights:

WCB

Gross Volumes:

Gross Heights:
JUM

JUL

AUG

SEP

ocr

Nov

DEC

$6.25 E-01$
$5.51 E+05$

6.25E-01

\section{$2.32 E+02$}

$2.14 E+02$
$2.35 E+08$

$1.37 E+02$

$1.98 E+00$

7.57E- 02

4. $47 E-01$

$3.40 E+04$

$5.01 E+05$

$1.58 E+01$

$1.33 \mathrm{E}+07$

7.38E-01

$1.69 E+00$

1.51E-01

$2.65 E-01$
$1.63 E+05$

$1.04 E+00$

$\begin{array}{ll}3.03 E-01 & 4.16 E-01 \\ 8.85 E+04 & 5.31 E+05\end{array}$

$3.18 E+04$

$8.57 E+05$

1.51E-01

$5.78 E+05$

$7.47 E+05$

$6.06 E+02$

$1.89 E-02$

$1.09 E+04$

$9.30 E+04$

$1.14 E-01$
$3.19 E+04$

4.66E- 01

4. $19 E+05$

2.65E+06

5.76E+04 6.85E+04

2.27E-01 2.27E-01

$8.94 E+04 \quad 8.94 E+04$

2.11E-01 1.08E+01

$\begin{array}{lll}1.36 E+00 & 1.14 E-01 & 1.78 E+00\end{array}$

$1.09 E+06 \quad 2.61 E+06$

2.87E+00

$1.70 E+06$

$5.42 E+05$

$7.76 E+04$

$3.24 E+00 \quad 6.89 E+00$

2.10E+00 $\quad 1.51 E+00$

$\begin{array}{llll}1.40 E+06 & 1.68 E+06 & 2.18 E+06 & 4.96 E+06\end{array}$

$2.67 E+00$

2.08E-01

2.08E-01

$4.54 E+04$

$5.26 E+04$

$3.46 E+00$

$4.08 E+05$

6.28E-01

$1.97 E+05$

3.41E-01

$1.88 E+05$

$5.68 E-02$

$2.22 E+04$

3. 79E-02

$1.40 E+04$ $\begin{array}{ll}2.11 E-01 & 1.08 E+01 \\ 1.27 E+05 & 5.16 E+06\end{array}$

$2.73 E+00 \quad 2.65 E+01$

$1.79 E+06 \quad 1.93 E+07$

3.92E+05 4.90E+05

$\begin{array}{ll}1.87 E+00 & 5.34 E+00\end{array}$

$6.28 E-01$

$6.28 E-01$
$1.97 E+05$

$9.14 E+00 \quad 1.00 E+01$ 6.65E+06 7.12E+06

2.27E-01 $1.83 E+02$

$1.36 E+05 \quad 1.30 E+08$

3. $79 E-02$

3.03E-01 3.03E-01

$2.07 E+05 \quad 2.07 E+05$
2.55E-01 6.71E-01 
INEL MONRADIOLOGICAL WASTE MAMAGEMENT IMFORMATION SYSTEM

SUMWARY INEL

HAZARDQUS MASTE GEMERATED

RPT 10-I

FOR January - Decenber 1992

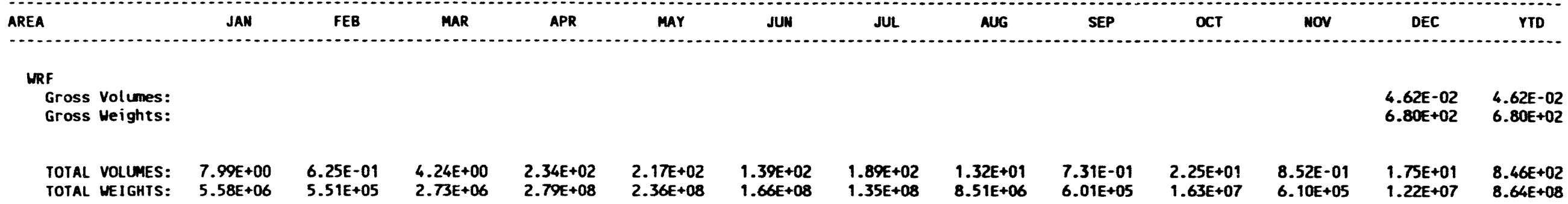

$\bar{Z}$
$\frac{1}{5}$
$\frac{1}{a}$

NOTE: VOLUME REPORTED IN CUBIC METERS; VEIGHT REPORTED IN GRAMS DETAILS MAY MOT ADD UP TO TOTALS BECMUSE OF ROMMDIMG 
Page: 1

IDAHO OPERATIONS OFFICE

19- J0N-93

Sched. Mo. INRPTO14A

UNITED STATES DEPARTMENT OF EMERGY

INEL NONRADIOLOGICAL MASTE MAMAGEMEMT IMFORMATION SYSTEM

SUMMARY INEL

MIXED MASTE GEMERATED

FR January - Decenaber 1992

RPT 11-1

AREA

JAN

FEB

MAR

APR

maY

JUN

JUL

AUG

SEP

OCT

nov

DEC
Gross Volumes:

Gross Weights:

CFA

Gross Volumes:

Gross Weights:

CPP

Gross Volumes:

Gross Weights:

IRC

Coss Volumes:

Gross Weights:

$=\mathrm{PBF}$

Gross Volumes:

Gross Weights:

SLR

Gross Volumes:

Gross Weights:

TAN

Gross Weights:

TRA

Gross Volumes:

Gross Weights:

WER

Gross Volumes:

Gross Weights:

WMC

Gross volumes:

Gross Heights:

TOTAL VOLUMES:

TOTAL WEIGHTS:
Gross Volumes:

\subsection{E-01}

$5.37 E+05$

5.98E-01

4. 19E+05

2. $19 E+00$

$6.79 E+05$

3.22E-01

$2.30 E+05$

3.03E-02

$4.35 E+04$

\section{$1.26 E+00$}

$6.46 E+05$

8.33E-01

8.10E+05

$\begin{array}{ll}3.60 E-01 & 3.70 E+01 \\ 8.62 E+04 & 6.06 E+06\end{array}$

$3.96 E+00$

$1.78 E+06$

\section{$6.28 E-01$}

$2.43 E+05$

8.33E-01

4.35E+00 4.24E+01

$1.91 E+06 \quad 8.82 E+06$

$\begin{array}{lll} & & 4.16 E-01 \\ & 2.38 E-01 & 5.37 E+05 \\ 6.30 E+04 & 8.37 E-01 \\ & & 4.82 E+05 \\ & & 2.19 E+00 \\ & & 6.79 E+05 \\ & & 3.22 E-01 \\ 8.33 E-01 & & 2.30 E+05 \\ 8.10 E+05 & & 3.03 E-02 \\ & & 4.35 E+04 \\ & & 1.26 E+00 \\ 8.33 E-01 & & 6.46 E+05 \\ & & 8.33 E-01 \\ & & 8.10 E+05 \\ & & 3.73 E+01 \\ & & 6.15 E+06 \\ & & 3.96 E+00 \\ & & 1.78 E+06 \\ & & 6.28 E-01 \\ & & 2.43 E+05 \\ & & 4.78 E+01 \\ & & 1.16 E+07\end{array}$

MOTE: VOLUME REPORTED IN CUBIC METERS; WEIGHT REPORTED IN GRAMS DETAILS MAY NOT NDC UP TO TOTALS BECAUSE OF ROUNDIMG 
ARGONNE NATIONAL LABORATORY-WEST, 1992 DETAIL DATA

Report 114-ANL Airborne Disposed Substances Summary ............ ANL-3

Report 114-ANL Airborne Release Point And Disposed Substances

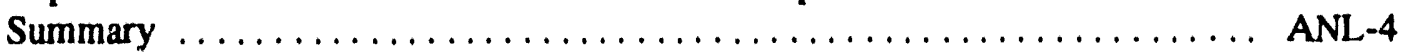

Report 115-ANL Liquid Disposed Substances Summary . . . . . . . . . ANL-5

Report 115-ANL Release Point And Liquid Disposed Substances

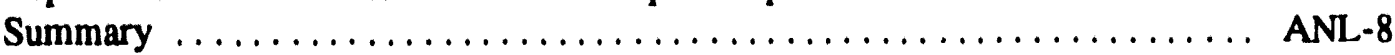

Report 116-ANL Landfill Solid Disposed Waste Summary . . . . . . . . . ANL-12

Report 117-ANL Water Usage And Disposal Summary ............. ANL-13

Report 118-ANL Fuel Oil Usage And Stack Effluents Summary ......... ANL-15

Report 1110-Hazardous Waste Generated By ANL For January-December

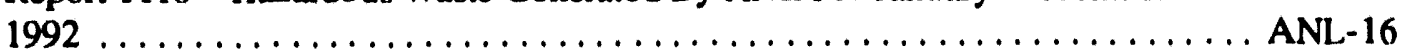

Report 1111-Mixed Waste Generated By ANL For January-December 1992 ANL-17 
PAGE: 1

IDAHO OPERATIONS OFFICE

ANL AIRBORME DISPOSED SUBSTANCES SUMMARY

SUBSTANCE

BET2 20K

MEIGHT (KGS)

POLYACRYLATE

WEIGHT (KGS)

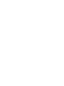

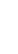

$\begin{array}{llllllll}1 & 7 & 9 & 2 & 7 & 8 & 7 & 46 \\ 0 & 0 & 1 & 0 & 0 & 1 & 0 & 3\end{array}$

DETAILS MAY NOT ADD UP TO TOTALS BECAUSE OF ROUNDING

0 IN A COLUMA IMDICATES $A$ TOTAL OF $<0.5$ 
PAGE: 1

SCHED. NO. INRPTOOT
IDAHO OPERATIONS OFFICE

UNITED STATES DEPARTMENT OF ENERGY

INEL NOMRADIOLOGICAL WASTE MAMAGEMENT IMFORMATION SYSTEM

FOR JAMUARY THROUGH DECEMBER 1992

ANL AIRBORNE RELEASE POINT AND DISPOSED SUBSTAMCES SUMAARY

RPT 114-1

ANL

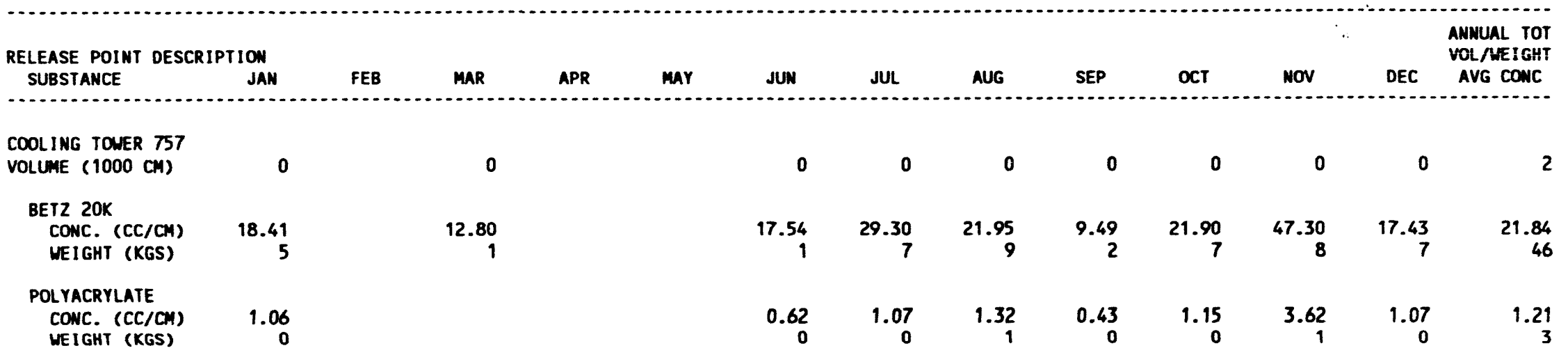


PAGE: 1

SCHED. MO. INRPTOOSA
IOAMO OPERATIONS OFFICE

$25-$ JUN-93

UMITED STATES DEPARTMENT OF EMERGY

IMEL MONRADIOLOGICAL MASTE MAMAGEMENT INFORMATION SYSTEM

FOR JANUARY THROUGH DECEMBER 1992

ANL LIQUID DISPOSED SUBSTANCES SUMURY

RPT 115-1

ANL

\begin{tabular}{|c|c|c|c|c|c|c|c|c|c|c|c|c|c|}
\hline SUBSTANCE & JAN & FEB & MAR & APR & mar & JUN & JUL & AUG & SEP & OCT & MON & DEC & $\begin{array}{l}\text { ANNUAL } \\
\text { TOTALS }\end{array}$ \\
\hline \multicolumn{14}{|c|}{ 1-BROMO-3-CHLORO-5,5-DIMETHYLHYDANT } \\
\hline $\begin{array}{l}\text { AMINEMETHYLPROP } \\
\text { WEIGHT (KGS) }\end{array}$ & 1 & 0 & 1 & & 3 & 2 & 1 & 1 & 1 & 2 & 1 & 2 & 15 \\
\hline $\begin{array}{l}\text { AROMATIC SOLVEN } \\
\text { WEIGHT (KGS) }\end{array}$ & 6 & 17 & 17 & 3 & 2 & 22 & 13 & 18 & 11 & 22 & 13 & 24 & 168 \\
\hline $\begin{array}{l}\text { 8-BROMO-B-NITRO } \\
\text { WEIGHT (KGS) }\end{array}$ & & 2 & 2 & 1 & 0 & 5 & 3 & 3 & 2 & 5 & 3 & 5 & 30 \\
\hline $\begin{array}{l}\text { BET2 } 20 K \\
\text { MEIGHT (KGS) }\end{array}$ & 430 & & 105 & 11 & 99 & 215 & 622 & 1,272 & 292 & 661 & 628 & 556 & 4,890 \\
\hline $\begin{array}{l}\text { BIS(TRICHLOROME } \\
\text { WEIGHT (KGS) }\end{array}$ & $\begin{array}{r}\text { FONE } \\
7\end{array}$ & 7 & 7 & & 1 & & & 6 & & & & & 28 \\
\hline $\begin{array}{l}\text { CARBOXYALKYLATE } \\
\text { WEIGHT (KGS) }\end{array}$ & ACCHAR & & & & & & & 0 & 0 & 0 & 0 & & 1 \\
\hline $\begin{array}{l}\text { CARBOXYLATED LI } \\
\text { WEIGHT (KGS) }\end{array}$ & & & & & & & & 0 & 0 & 0 & 0 & & 0 \\
\hline $\begin{array}{l}\text { CYCLOHEXYLAMIME } \\
\text { WEIGHT (KGS) }\end{array}$ & & & & & & & & & 0 & 1 & 1 & 0 & 2 \\
\hline $\begin{array}{l}\text { DIMETHYLI SOPROP } \\
\text { WEIGHT (KGS) }\end{array}$ & VE & 0 & 1 & & 3 & 2 & $?$ & 1 & 1 & 2 & 2 & 2 & 16 \\
\hline $\begin{array}{l}\text { ETHYLAMINE } \\
\text { WEIGHT (KGS) }\end{array}$ & 0 & 0 & 0 & & 0 & 1 & 0 & 0 & 0 & 0 & 0 & 0 & 2 \\
\hline $\begin{array}{l}\text { HEAVY AROMATIC } \\
\text { WEIGHT (KGS) }\end{array}$ & 5 & 5 & 5 & & 1 & & & 4 & & & & & 21 \\
\hline $\begin{array}{l}\text { HYDROGEN CHLORI } \\
\text { WEIGHT (KGS) }\end{array}$ & 5 & 5 & 5 & 5 & 5 & 5 & 5 & 5 & 5 & 5 & 5 & & 52 \\
\hline
\end{tabular}

DETAILS MAY NOT ADD UP TO TOTALS BECAUSE OF ROUNDIMG

0 IN A COLUNA INDICATES $A$ TOTAL OF $<0.5$ 
PAGE: 2

SCHED. NO. INRPTOOSA

IDAHO OPERATIONS OFFICE

25- JUN-93

UNITED STATES DEPARTMENT OF EMERGY

IMEL MOMRADIOLOGICAL MASTE MAMAGEMEMT INFORMATIOW SYSTEM

FOR JAMUARY THROUGH DECEMBER 1992

ANL LIQUID DISPOSED SUBSTANCES SUMMARY

RPT $195-1$

SUBSTANCE
ISOPROPANOL

WEIGHT (KGS)

2

2

2

JANITORIAL SUPPLIES

WEIGHT (KGS)

METHYLEME BIS(THIOCYAMATE)

WEIGHT (KGS)

N-METHYLPYRROL IDONE

WEIGHT (KGS)

1

MONYLPHENOXYPOLY(ETHYLENEOXY)ETHANOL

WEIGHT (KGS)

2

3

PHOTO LAB CHEMICAL MEIGHT (KGS)

POLYACRYLATE

WEIGHT (KGS)

$25 \quad 11$

POLYALKYLENE GLYCOL

WEIGHT (KGS)

SALT OF EDTA

VEIGHT (KGS)

SOOIUN HYDRATE SOLUTION WEIGHT (KGS)

SOOIUM ION

WEIGHT (KGS)

374

415

415

83

332

\begin{tabular}{|c|c|c|c|c|c|}
\hline & & & & 291 & 291 \\
\hline 2 & 1 & 2 & 1 & 2 & 19 \\
\hline 10 & 4 & 7 & 4 & 8 & $\sqrt{5}$ \\
\hline \multirow[t]{2}{*}{3} & 1 & 2 & 1 & 2 & 21 \\
\hline & & 70 & & 460 & 792 \\
\hline 76 & 13 & 35 & 48 & 34 & 280 \\
\hline \multirow[t]{2}{*}{0} & 0 & 0 & 0 & & 0 \\
\hline & 0 & 0 & 0 & 0 & 0 \\
\hline 1 & 1 & 3 & 1 & 0 & 8 \\
\hline 291 & 332 & 415 & 416 & 390 & 4,085 \\
\hline 0 & 0 & 0 & 0 & & 1 \\
\hline 0 & 1 & 2 & 0 & & 4 \\
\hline
\end{tabular}

SOOIUM SULFATE

MEIGHT (KGS)

SCDIUN SULFITE

WEIGHT (KGS) 
PAGE: 3

AML LIOUID DISPOSED SUBSTANCES SUMMARY

RPT 115-I

ANL

\begin{tabular}{|c|c|c|c|c|c|c|c|c|c|c|c|c|c|}
\hline SUBSTANCE & JAN & FEB & MAR & APR & Mar & JUN & JUL & AUG & SEP & OCT & NOV & DEC & $\begin{array}{l}\text { ANNUAL } \\
\text { TOTALS }\end{array}$ \\
\hline $\begin{array}{l}\text { SOOIUN TRIPOLYP } \\
\text { WEIGHT (KGS) }\end{array}$ & & & & & & & & 0 & 0 & 1 & 0 & & 1 \\
\hline $\begin{array}{l}\text { STODDARD SOLVEN } \\
\text { WEIGHT (KGS) }\end{array}$ & & 6 & 6 & 2 & 1 & 13 & 7 & 7 & 6 & 13 & 7 & 14 & 83 \\
\hline $\begin{array}{l}\text { SULFATE ION } \\
\text { WEIGHT (KGS) }\end{array}$ & 4,361 & 1,108 & 1,800 & 208 & 1,038 & 2,077 & 4,431 & 7,961 & 2,215 & 4,912 & 1,411 & 4,912 & 36,434 \\
\hline $\begin{array}{l}\text { SULFITE ION } \\
\text { WEIGHT (KGS) }\end{array}$ & & & & & & & & 0 & 0 & 1 & 1 & 0 & 3 \\
\hline
\end{tabular}


PAGE: 1

SCHED. NO. INRPTOOS
IDAHO OPERATIONS OFFICE

25- JUN-93

UNITED STATES DEPARTMENT OF ENERGY

INEL NOWRADIOLOGICAL UASTE MAMAGEMENT INFORMATION SYSTEM

FOR JAMUARY THROUGH DECEMBER 1992

ANL RELEASE POINT AND LIQUID DISPOSED SUBSTANCES SUMmARY

RPT 115-I

ANL

\begin{tabular}{|c|c|c|c|c|c|c|c|c|c|c|c|c|c|}
\hline $\begin{array}{l}\text { RELEASE POINT DESCRIP } \\
\text { SUBSTANCE }\end{array}$ & TION JAN & FEB & MAR & APR & mar & JUN & JUL & AUG & SEP & OCT & NOV & DEC & $\begin{array}{l}\text { ANNUAL TOT } \\
\text { VOL/WEIGHT } \\
\text { AVG CONC }\end{array}$ \\
\hline $\begin{array}{l}\text { FACILITY } 779 \\
\text { VOLUME }(1000 \mathrm{~L}) \\
\text { PH }\end{array}$ & $\begin{array}{r}\text { SURFACE } \\
908\end{array}$ & 816 & 971 & 955 & 905 & 1,022 & 1,233 & 1,007 & 1,117 & 1,149 & 964 & 1,362 & 12,407 \\
\hline $\begin{array}{l}\text { HYDROGEN CHLORIDE } \\
\text { CONC. (MG/L) } \\
\text { WEIGHY (KGS) }\end{array}$ & $\begin{array}{r}5.24 \\
5\end{array}$ & $\begin{array}{r}5.84 \\
5\end{array}$ & $\begin{array}{r}4.91 \\
5\end{array}$ & $\begin{array}{r}4.99 \\
5\end{array}$ & $\begin{array}{r}5.26 \\
5\end{array}$ & $\begin{array}{r}4.66 \\
5\end{array}$ & $\begin{array}{r}3.86 \\
5\end{array}$ & $\begin{array}{r}4.73 \\
5\end{array}$ & $\begin{array}{r}4.26 \\
5\end{array}$ & $\begin{array}{r}4.15 \\
5\end{array}$ & $\begin{array}{r}4.94 \\
5\end{array}$ & & $\begin{array}{r}4.22 \\
52\end{array}$ \\
\hline $\begin{array}{l}\text { ISOPROPANOL } \\
\text { CONC. (MG/L) } \\
\text { WEIGHT (KGS) }\end{array}$ & $\begin{array}{r}2.62 \\
2\end{array}$ & $\begin{array}{r}2.92 \\
2\end{array}$ & $\begin{array}{r}2.45 \\
2\end{array}$ & $\begin{array}{r}2.49 \\
2\end{array}$ & $\begin{array}{r}2.63 \\
2\end{array}$ & $\begin{array}{r}2.33 \\
2\end{array}$ & $\begin{array}{r}1.93 \\
2\end{array}$ & $\begin{array}{r}2.37 \\
2\end{array}$ & $\begin{array}{r}2.13 \\
2\end{array}$ & $\begin{array}{r}2.07 \\
2\end{array}$ & $\begin{array}{r}2.47 \\
2\end{array}$ & & $\begin{array}{r}2.11 \\
26\end{array}$ \\
\hline $\begin{array}{l}\text { JANITORIAL SUPPLIE: } \\
\text { CONC. (MG/L) } \\
\text { WEIGHT (KGS) }\end{array}$ & & & & & & & & & & & & $\begin{array}{r}213.90 \\
291\end{array}$ & $\begin{array}{r}23.48 \\
291\end{array}$ \\
\hline $\begin{array}{l}\text { INDUSTRIAL POND } 746 \\
\text { VOLUME }(1000 \text { L) } \\
\text { PH }\end{array}$ & $\begin{array}{l}\text { SURFACE } \\
12,454\end{array}$ & 8,362 & 10,565 & 5,568 & 7,745 & 13,968 & 30,378 & 22,803 & 11,977 & 21,914 & 7,949 & 11,530 & 165,214 \\
\hline $\begin{array}{l}\text { 1-BROMO-3-CHLORO-5 } \\
\text { CONC. (MG/L) } \\
\text { WEIGHT (KGS) }\end{array}$ & $\begin{array}{r}\text { 5-DIMETHY } \\
4.04 \\
50\end{array}$ & $\begin{array}{r}3.01 \\
25\end{array}$ & $\begin{array}{r}3.57 \\
38\end{array}$ & $\begin{array}{r}0.75 \\
4\end{array}$ & $\begin{array}{r}1.08 \\
8\end{array}$ & $\begin{array}{r}2.70 \\
38\end{array}$ & $\begin{array}{r}3.45 \\
105\end{array}$ & $\begin{array}{r}13.43 \\
306\end{array}$ & $\begin{array}{r}8.76 \\
105\end{array}$ & $\begin{array}{r}5.32 \\
117\end{array}$ & $\begin{array}{r}5.28 \\
42\end{array}$ & $\begin{array}{r}9.97 \\
115\end{array}$ & $\begin{array}{r}5.77 \\
953\end{array}$ \\
\hline $\begin{array}{l}\text { AMINERE THTLPROFARO } \\
\text { CONC. (MG/L) } \\
\text { MEIGHT (KGS) }\end{array}$ & $\begin{array}{r}0.09 \\
1\end{array}$ & $\begin{array}{r}0.02 \\
0\end{array}$ & $\begin{array}{r}0.06 \\
1\end{array}$ & & $\begin{array}{r}0.41 \\
3\end{array}$ & $\begin{array}{r}0.15 \\
2\end{array}$ & $\begin{array}{r}0.03 \\
1\end{array}$ & $\begin{array}{r}0.06 \\
1\end{array}$ & $\begin{array}{r}0.07 \\
1\end{array}$ & $\begin{array}{r}0.07 \\
2\end{array}$ & $\begin{array}{r}0.17 \\
1\end{array}$ & $\begin{array}{r}0.15 \\
2\end{array}$ & $\begin{array}{r}0.09 \\
15\end{array}$ \\
\hline $\begin{array}{l}\text { AROMATIC SOLVENTS } \\
\text { CONC. (MG/L) } \\
\text { WEIGHT (KGS) }\end{array}$ & $\begin{array}{r}0.46 \\
6\end{array}$ & $\begin{array}{r}2.02 \\
17\end{array}$ & $\begin{array}{r}1.60 \\
17\end{array}$ & $\begin{array}{r}0.57 \\
3\end{array}$ & $\begin{array}{r}0.32 \\
2\end{array}$ & $\begin{array}{r}1.60 \\
22\end{array}$ & $\begin{array}{r}0.42 \\
13\end{array}$ & $\begin{array}{r}0.77 \\
18\end{array}$ & $\begin{array}{r}0.93 \\
11\end{array}$ & $\begin{array}{r}1.02 \\
22\end{array}$ & $\begin{array}{r}1.61 \\
13\end{array}$ & $\begin{array}{r}2.08 \\
24\end{array}$ & $\begin{array}{r}1.02 \\
168\end{array}$ \\
\hline
\end{tabular}




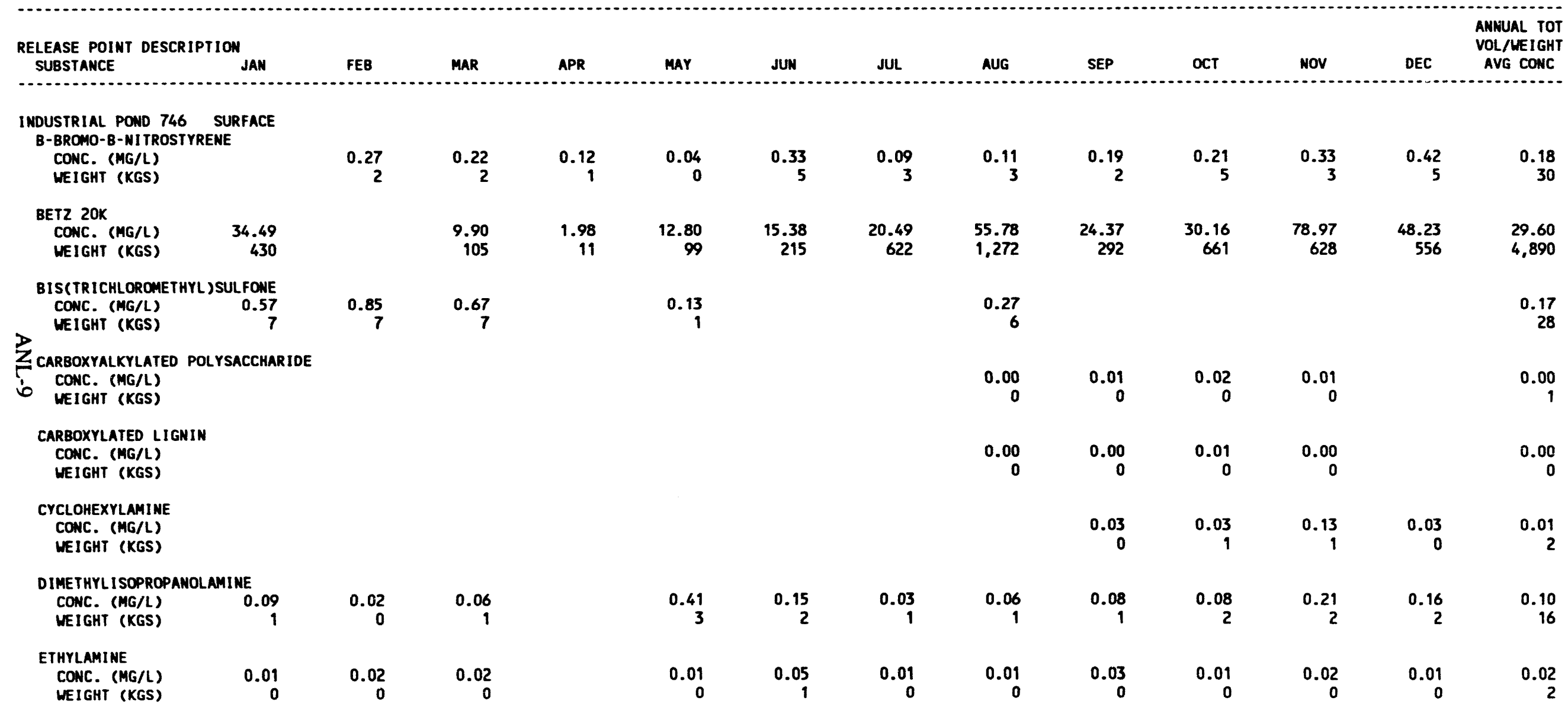


PAGE: 3

SCHED. NO. INRPTOOS
IDAHO OPERATIONS OFFICE

25- JUN-93

UNITED STATES DEPARTMENT OF ENERGY

INEL NONRADIOLOGICAL YASTE MAMAGEMENT INFORMATION SYSTEM

FOR JAMUARY THROUGH DECEMBER 1992

ANL RELEASE POINT AND LIOUIC DISPOSED SUBSTANCES SUMMARY

RPT 115-I

ANL

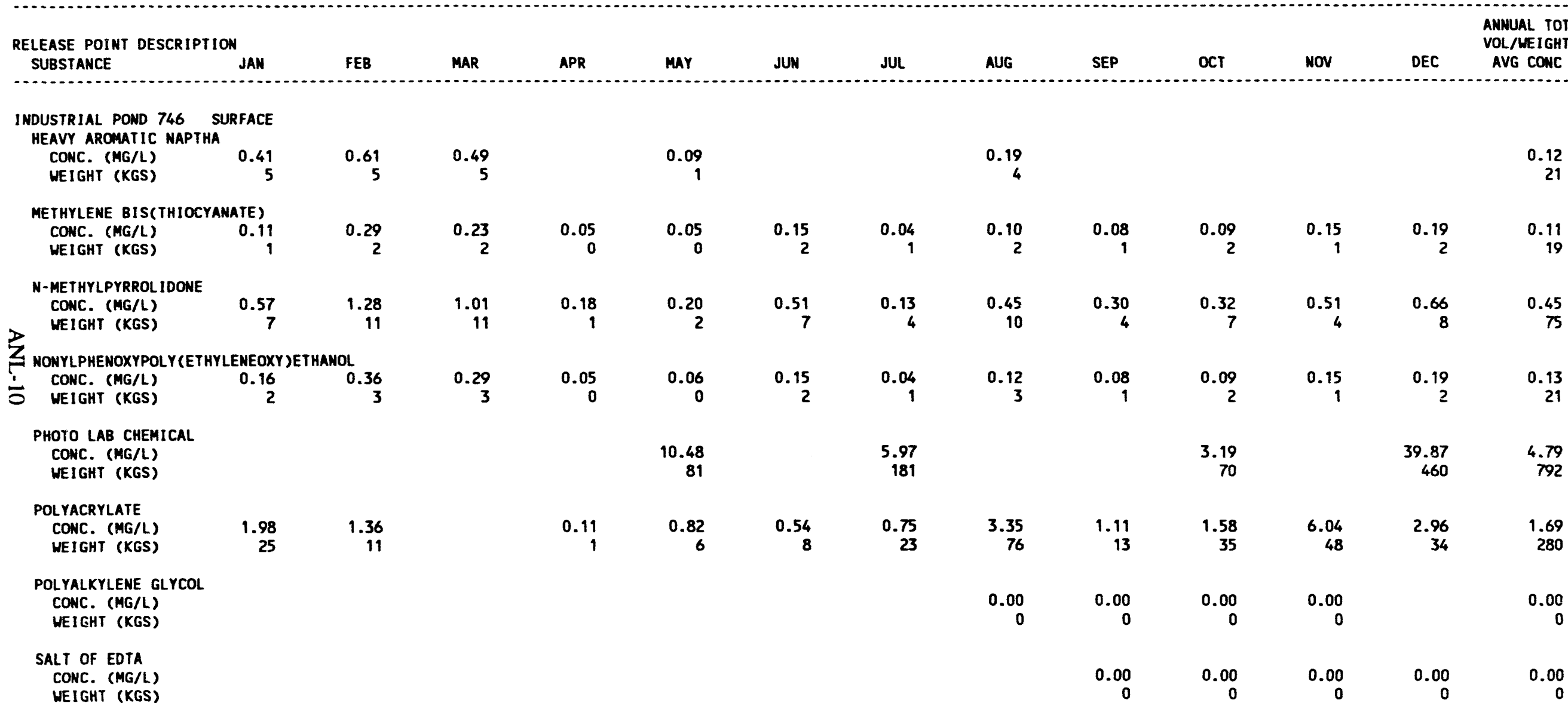

DETAILS MAY NOT ADD UP TO TOTALS BECAUSE OF ROUNDING

O IN A COLUMM INDICATES A TOTAL OF $<0.5$

0.00 IN A COLUMM IMDICATES A TOTAL OF $<0.005$ 


\begin{tabular}{|c|c|c|c|c|c|c|c|c|c|c|c|c|}
\hline $\begin{array}{l}\text { RELEASE POINT DESCRIPTION } \\
\text { SUBSTANCE JAN }\end{array}$ & FEB & MAR & APR & MAY & JUN & JUL & AUG & SEP & OCT & NOV & DEC & $\begin{array}{l}\text { ANNUAL TOT } \\
\text { VOL/WEIGHT } \\
\text { AVG CONC }\end{array}$ \\
\hline $\begin{array}{l}\text { INDUSTRIAL POND } 746 \text { SURFACE } \\
\text { SODIUM HYDRATE SOLUTION } \\
\text { CONC. (MG/L) } \\
\text { WEIGHT (KGS) }\end{array}$ & & & & & & & $\begin{array}{r}0.06 \\
1\end{array}$ & $\begin{array}{r}0.11 \\
1\end{array}$ & $\begin{array}{r}0.16 \\
3\end{array}$ & $\begin{array}{r}0.15 \\
1\end{array}$ & $\begin{array}{r}0.02 \\
0\end{array}$ & $\begin{array}{r}0.05 \\
8\end{array}$ \\
\hline $\begin{array}{l}\text { SODIUM ION } \\
\text { CONC. (MG/L) } \\
\text { WEIGHT (KGS) }\end{array}$ & $\begin{array}{r}49.63 \\
415\end{array}$ & $\begin{array}{r}39.28 \\
415\end{array}$ & $\begin{array}{r}14.91 \\
83\end{array}$ & $\begin{array}{r}42.87 \\
332\end{array}$ & $\begin{array}{r}11.89 \\
166\end{array}$ & $\begin{array}{r}15.02 \\
456\end{array}$ & $\begin{array}{r}12.74 \\
291\end{array}$ & $\begin{array}{r}27.74 \\
332\end{array}$ & $\begin{array}{r}18.96 \\
415\end{array}$ & $\begin{array}{r}52.29 \\
416\end{array}$ & $\begin{array}{r}33.85 \\
390\end{array}$ & $\begin{array}{l}24.73 \\
4.085\end{array}$ \\
\hline $\begin{array}{l}\text { SOD IUM SULFATE } \\
\text { CONC. (MG/L) } \\
\text { WEIGHT (KGS) }\end{array}$ & & & & & & & $\begin{array}{r}0.00 \\
0\end{array}$ & $\begin{array}{r}0.01 \\
0\end{array}$ & $\begin{array}{r}0.02 \\
0\end{array}$ & $\begin{array}{r}0.01 \\
0\end{array}$ & & $\begin{array}{r}0.00 \\
1\end{array}$ \\
\hline $\begin{array}{l}Z \text { SOOIUM SULFITE } \\
\quad \text { CONC. (MG/L) } \\
\vdots \quad \text { WEIGHT (KGS) }\end{array}$ & & & & & & & $\begin{array}{r}0.01 \\
0\end{array}$ & $\begin{array}{r}0.08 \\
1\end{array}$ & $\begin{array}{r}0.10 \\
2\end{array}$ & $\begin{array}{r}0.03 \\
0\end{array}$ & & $\begin{array}{r}0.02 \\
4\end{array}$ \\
\hline $\begin{array}{l}\text { SOOIUM TRIPOLYPHOSPHATE } \\
\text { CONC. (MG/L) } \\
\text { WEIGHT (KGS) }\end{array}$ & & & & & & & $\begin{array}{r}0.01 \\
0\end{array}$ & $\begin{array}{r}0.03 \\
0\end{array}$ & $\begin{array}{r}0.04 \\
1\end{array}$ & $\begin{array}{r}0.01 \\
0\end{array}$ & & $\begin{array}{r}0.01 \\
1\end{array}$ \\
\hline $\begin{array}{l}\text { STOODARD SOLVENT } \\
\text { CONC. (MG/L) } \\
\text { WEIGHT (KGS) }\end{array}$ & $\begin{array}{r}0.76 \\
6\end{array}$ & $\begin{array}{r}0.60 \\
6\end{array}$ & $\begin{array}{r}0.33 \\
2\end{array}$ & $\begin{array}{r}0.12 \\
1\end{array}$ & $\begin{array}{r}0.91 \\
13\end{array}$ & $\begin{array}{r}0.24 \\
7\end{array}$ & $\begin{array}{r}0.32 \\
7\end{array}$ & $\begin{array}{r}0.53 \\
6\end{array}$ & $\begin{array}{r}0.58 \\
13\end{array}$ & $\begin{array}{r}0.91 \\
7\end{array}$ & $\begin{array}{r}1.18 \\
14\end{array}$ & $\begin{array}{r}0.50 \\
83\end{array}$ \\
\hline $\begin{array}{l}\text { SULFATE ION } \\
\text { CONC. (MG/L) } \\
\text { WEIGHT (KGS) }\end{array}$ & $\begin{array}{r}132.47 \\
1,108\end{array}$ & $\begin{array}{r}170.36 \\
1,800\end{array}$ & $\begin{array}{r}37.30 \\
208\end{array}$ & $\begin{array}{r}134.06 \\
1,038\end{array}$ & $\begin{array}{r}148.69 \\
2,077\end{array}$ & $\begin{array}{r}145.85 \\
4,431\end{array}$ & $\begin{array}{r}349.10 \\
7,961\end{array}$ & $\begin{array}{r}184.97 \\
2,215\end{array}$ & $\begin{array}{r}224.17 \\
4,912\end{array}$ & $\begin{array}{r}177.46 \\
1,411\end{array}$ & $\begin{array}{r}426.04 \\
4.912\end{array}$ & $\begin{array}{l}220.53 \\
36,434\end{array}$ \\
\hline $\begin{array}{l}\text { SULFITE ION } \\
\text { CONC. (MG/L) } \\
\text { WEIGHT (KGS) }\end{array}$ & & & & & & & $\begin{array}{r}0.01 \\
0\end{array}$ & $\begin{array}{r}0.03 \\
0\end{array}$ & $\begin{array}{r}0.03 \\
1\end{array}$ & $\begin{array}{r}0.14 \\
1\end{array}$ & $\begin{array}{r}0.04 \\
0\end{array}$ & $\begin{array}{r}0.02 \\
3\end{array}$ \\
\hline
\end{tabular}


INEL NONRADIOLOGICAL UASTE MANAGEMENT INFORMATION SYSTEM

FOR JANUARY THROUGH DECEMBER 1992

ANL LANDFILL SOLID DISPOSED WASTE SUMMARY

RPT 116-1

ANL

(ALL VOLUMES = CUBIC METERS)

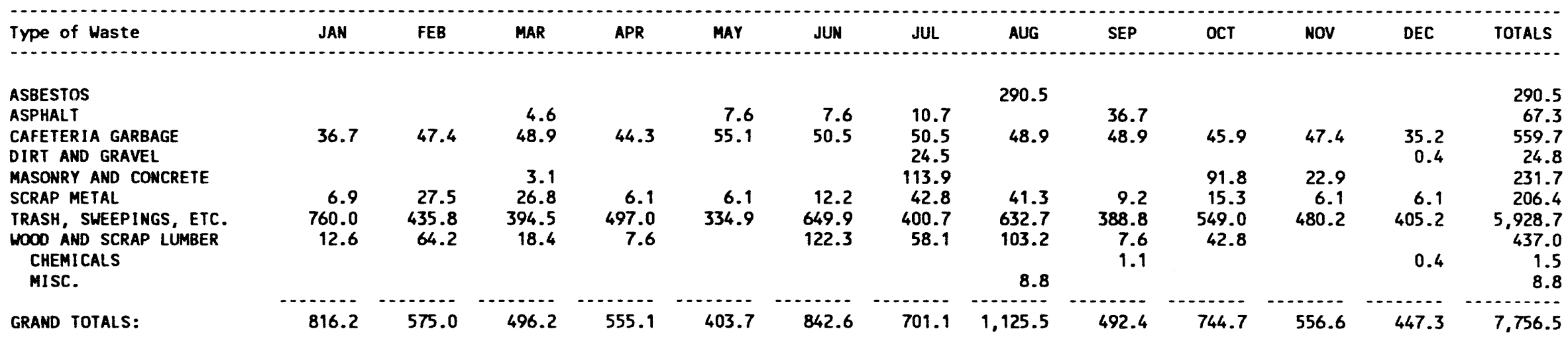

$\frac{2}{n}$ 
PAGE:

SCHED. NO. INRPTO10
IDAHO OPERATIONS OFFICE

UNITED STATES DEPARTMENT OF ENERG

INEL NONRADIOLOGICAL UASTE MANAGEMENT INFORMATION SYSTEM

FOR JANUARY THROUGH DECEMBER 1992

ANL WATER USAGE AND DISPOSAL SUMARAR

RPT $117-1$

ANL

ANNUAL TOT

WELL OR BLDG. NO.

(ALL VOLUMES = NEAREST THOUSAND LITERS)
19- JUN-93
JAN

FEB

MAY

JUL

AUG

OCT DEC AVG CONC

\section{WATER DATA}

O01 WATER PUMPED (L)

002 WATER PUMPED (L)

WATER PUMPED (L)

\begin{tabular}{|c|c|c|c|c|c|c|c|c|c|c|c|c|}
\hline $\begin{array}{l}17,413 \\
17,413\end{array}$ & $\begin{array}{l}6,643 \\
6,643\end{array}$ & $\begin{array}{l}9,906 \\
9,906\end{array}$ & $\begin{array}{l}4,667 \\
4,667\end{array}$ & $\begin{array}{l}7,408 \\
7,408\end{array}$ & $\begin{array}{l}13,014 \\
13,014\end{array}$ & $\begin{array}{l}23,852 \\
23,852\end{array}$ & $\begin{array}{l}38,043 \\
38,043\end{array}$ & $\begin{array}{l}21,728 \\
21,728\end{array}$ & $\begin{array}{l}25,143 \\
25,143\end{array}$ & $\begin{array}{l}11,580 \\
11,580\end{array}$ & $\begin{array}{l}23.292 \\
23,292\end{array}$ & $\begin{array}{l}202,689 \\
202,689\end{array}$ \\
\hline 34,826 & 13,287 & 19,813 & 9,335 & 14,816 & 26,028 & 47,704 & 76,087 & 43,456 & 50,285 & 23.159 & 46,583 & 405,378 \\
\hline
\end{tabular}

WATER DISPOSED

$$
\begin{aligned}
& \text { AIR (L) } \\
& \text { SURFACE (L) } \\
& \text { SUBSURFACE (L) }
\end{aligned}
$$

$\begin{array}{rrrr}19,673 & 3,397 & 7,241 & 2,297 \\ 12,454 & 8,362 & 10,565 & 5,568\end{array}$

\section{2,297}

$\begin{array}{llll}6,367 & 10,993 & 16,046 & 48,415\end{array}$

$28,144 \quad 24,666$

23,159

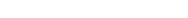

EUAGE DATA
45

45

BIOCHEMICAL OXYGEN DEMAND (BOD)

RAW CONC. (MG/L)

FINAL CONC. (MG/L)

X REMOVED

DISSOLVED OXYGEN (DO)

RAW CONC. (MG/L)

FINAL CONC. (MG/L)

INCREASED FACTOR

SETTLEABLE SOLIOS (SS)

RAW CONC. (ML/L)

FINAL CONC. (ML/L)

\% REMOVED

PH FINAL 
FOR JANUARY THROUGH DECEMBER 1992

ANL

(ALL VOLLMES = MEAREST THOUSAND LITERS)

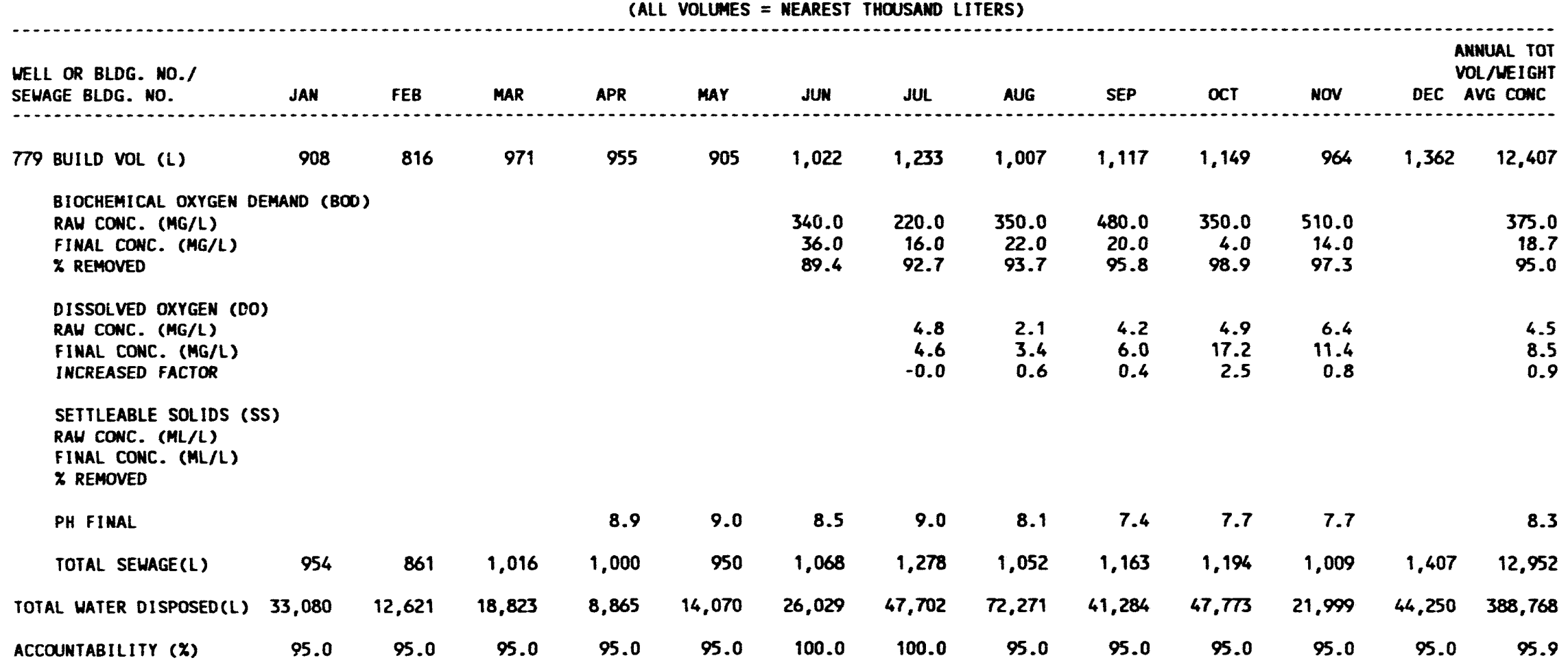


ANL FUEL OIL USAGE AMD STACK EFFLUENTS SUMHARY

(ALL VALUES = MEAREST THOUSAMD)

\begin{tabular}{|c|c|c|c|c|c|c|c|c|c|c|c|c|c|}
\hline $\begin{array}{l}\text { FUEL OIL TYPE } \\
\text { EFFLUENTS }\end{array}$ & JAN & FEB & MAR & APR & mar & JUN & JUL & AUG & SEP & OCT & MOV & DEC & $\begin{array}{l}\text { AMMUAL } \\
\text { TOTALS }\end{array}$ \\
\hline \multicolumn{14}{|l|}{ TYPE 2} \\
\hline VOLUME (LITERS) & 190 & 335 & 292 & 190 & 148 & 125 & 48 & 4 & 135 & 84 & 251 & 81 & 1,882 \\
\hline $\begin{array}{l}\text { SO2 (KGS) } \\
\text { NOX (KGS) }\end{array}$ & 1 & 2 & 2 & 1 & 1 & 1 & 0 & 0 & 1 & 0 & 1 & 0 & 10 \\
\hline PARTICULATES (KGS) & 0 & 0 & 0 & 0 & 0 & 0 & 0 & 0 & 0 & 0 & 0 & 0 & 2 \\
\hline \multicolumn{14}{|l|}{ GRAMD TOTALS: } \\
\hline VOLUME (LITERS) & 190 & 335 & 292 & 190 & 148 & 125 & 48 & 4 & 135 & 84 & 251 & 81 & 1,882 \\
\hline $\begin{array}{l}\text { SO2 (KGS) } \\
\text { NOX (KGS) }\end{array}$ & 1 & 2 & 2 & 1 & 1 & 1 & 0 & 0 & 1 & 0 & 1 & 0 & 10 \\
\hline PARTICULATES (KGS) & 0 & 0 & 0 & 0 & 0 & 0 & 0 & o & 0 & 0 & 0 & 0 & 2 \\
\hline
\end{tabular}


Page: 1
Sched. No. INRPT013

IDAHO OPERATIONS OFFICE

19- JUW-93

UWITED STATES DEPARTMENT OF ENERGY

INEL NONRADIOLOGICAL MASTE MAMAGEMENT INFORMATION SYSTEI

HAZARDOUS UASTE GENERATED BY AML

RPT 1110-I

FOR January - Deceaber 1992

ANL

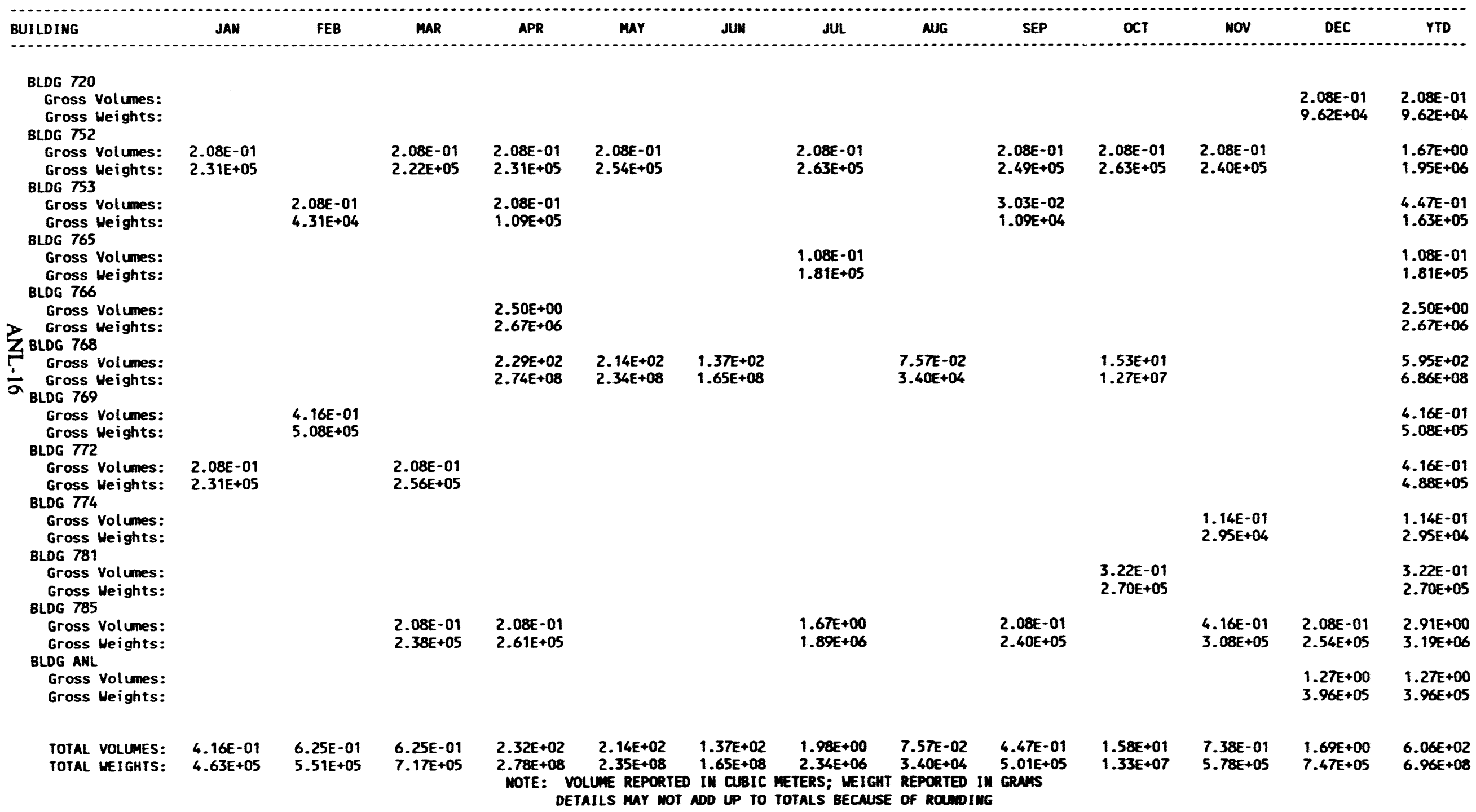


MIXED WASTE GENERATED BY AML

RPT 1111-

FOR January - December 1992

APR

Mar

JUN

JUL

AUG

SEP

OCT

MOV

DEC

YTD

BLDG 752

Gross Volumes:

Gross Weights:

TOTAL VOLUMES:

TOTAL MEIGHTS:

4.16E- 01

$5.37 E+05$

4. 16E-0

5.37E+05
4.16E-01

$5.37 \mathrm{E}+05$

4.16E-0

5.37E+05 


\section{CENTRAL FACILITIES AREA, 1992 DETAIL DATA}

Report 135-CFA Liquid Disposed Substances Summary ............ CFA-3

Report 135-CFA Release Point And Liquid Disposed Substances Summary . . CFA-5

Report 136-CFA Landfill Solid Disposed Waste Summary ........... CFA-8

Report 137-CFA Water Usage And Disposal Summary For

January Through December 1992

CFA-9

Report 138-CFA Fuel Oil Usage And Stack Effluents Summary . . . . . . . . CFA-11

Report 1310-Hazardous Waste Generated By CFA For January-December

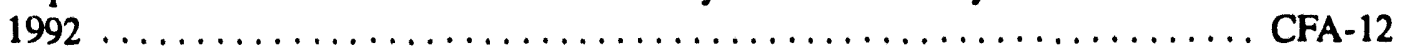

Report 1311-Mixed Waste Generated By CFA For January-December 1992 . . CFA-13 
PAGE: 1

SCHED. NO. INRPTOOBA
IDAHO OPERATIONS OFFICE

UNITED STATES DEPARTMENT OF EMERGY

UNITED STATES DEPARTMENT OF ENERGY

INEL NONRADIOLOGICAL WASTE MAMH

FOR JAMUARY THROUGH DECEMBER 1992

CFA LIQUID DISPOSED SUBSTAMCES SUMWARY

RPT 135-I

SUBSTANCE
ALUMINUM NITRATE
WEIGHT (KGS)
BARIUM SULFATE
WEIGHT (KGS)
CALCIUM CHLORIDE
WEIGHT (KGS)
CALCIUM FLUORIDE
WEIGHT (KGS)
CALCIUM NITRATE
WEIGHT (KGS)
CALCIUM PERCHLORATE
WEIGHT (KGS)

WEIGHT (KGS)

CALCIUM PHOSPHATE, TRIBASIC

WEIGHT (KGS)

JAN

FEB

MAR

APR

maY JUN JUL

JUL AUG

OCT

Nov

ANMUAL

CALCIUM SULFATE, AMHYDFOUS

WEIGHT (KGS)

CHLORIDE ION

CHLORIDE ION

WEIGHT (KGS)

DREWGARD
WEIGHT (KGS)

$$
1,541 \quad 1,252
$$

1,417

1,459

1,073

784

$984 \quad 1,459 \quad 1,583$

1,720

ETHYL ALCOHOL

2

5

WEIGHT (KGS)

22

ETHYLENEDIAMINE TETRAMCETIC ACID WEIGHT (KGS)

JANITORIAL SUPPLIES MEIGHT (KGS) 294

7

3

2
2
4
432

1,755

16,872

60

0

131

4

28

1

47

46

44

$345 \quad 319 \quad 743 \quad 5,710$

2

22

32

DETAILS MAY NOT ADD UP TO TOTALS BECAUSE OF ROUNDING 
PAGE: 2

SCHED. NO. INRPTOOBA
IDAHO OPERATIONS OFFICE

UNITED STATES DEPARTMENT OF ENERGY

INEL NONRADIOLOGICAL WASTE MANAGEMENT INFORMATION SYSTEM

FOR JAMUARY THROUGH DECEMBER 1992

CFA LIQUID DISPOSED SUBSTANCES SUMMARY

RPT 135-1

CFA

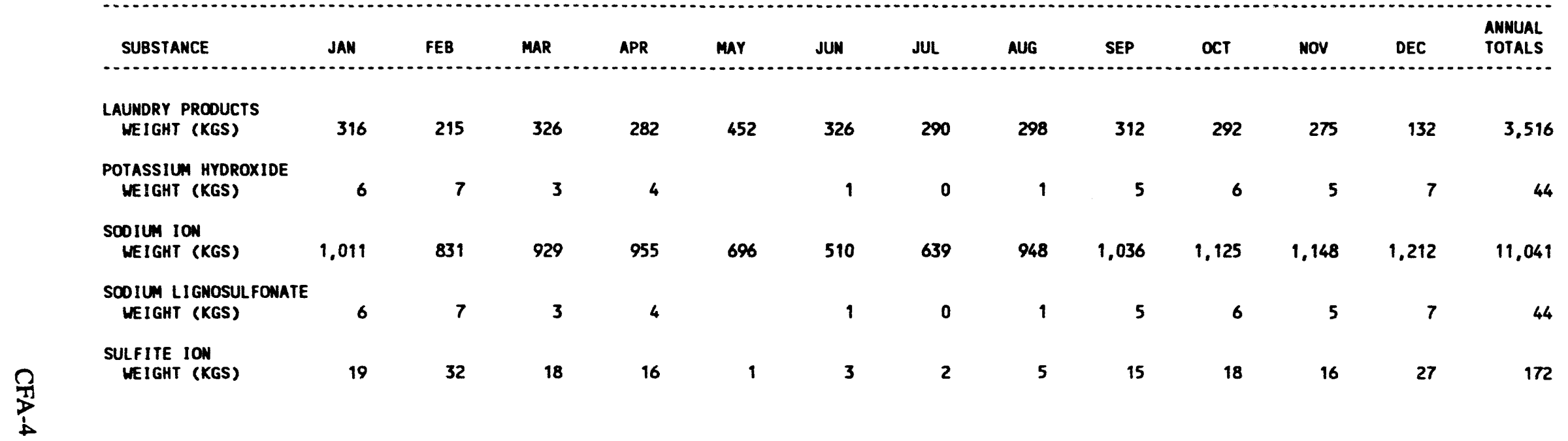


PAGE: 1

SCHED. NO. INRPT008
IDAHO OPERATIONS OFFICE

$25-J U N-93$

UNITED STATES DEPARTMENT OF EMERGY

INEL NONRAD IOLOGICAL WASTE MANAGEMENT INFORMATION SYSTEM

FOR JANUARY THROUGH DECEMBER 1992

CFA RELEASE POINT AND LIOUID DISPOSED SUBSTANCES SUMHARY

RPT 135- 1

CFA

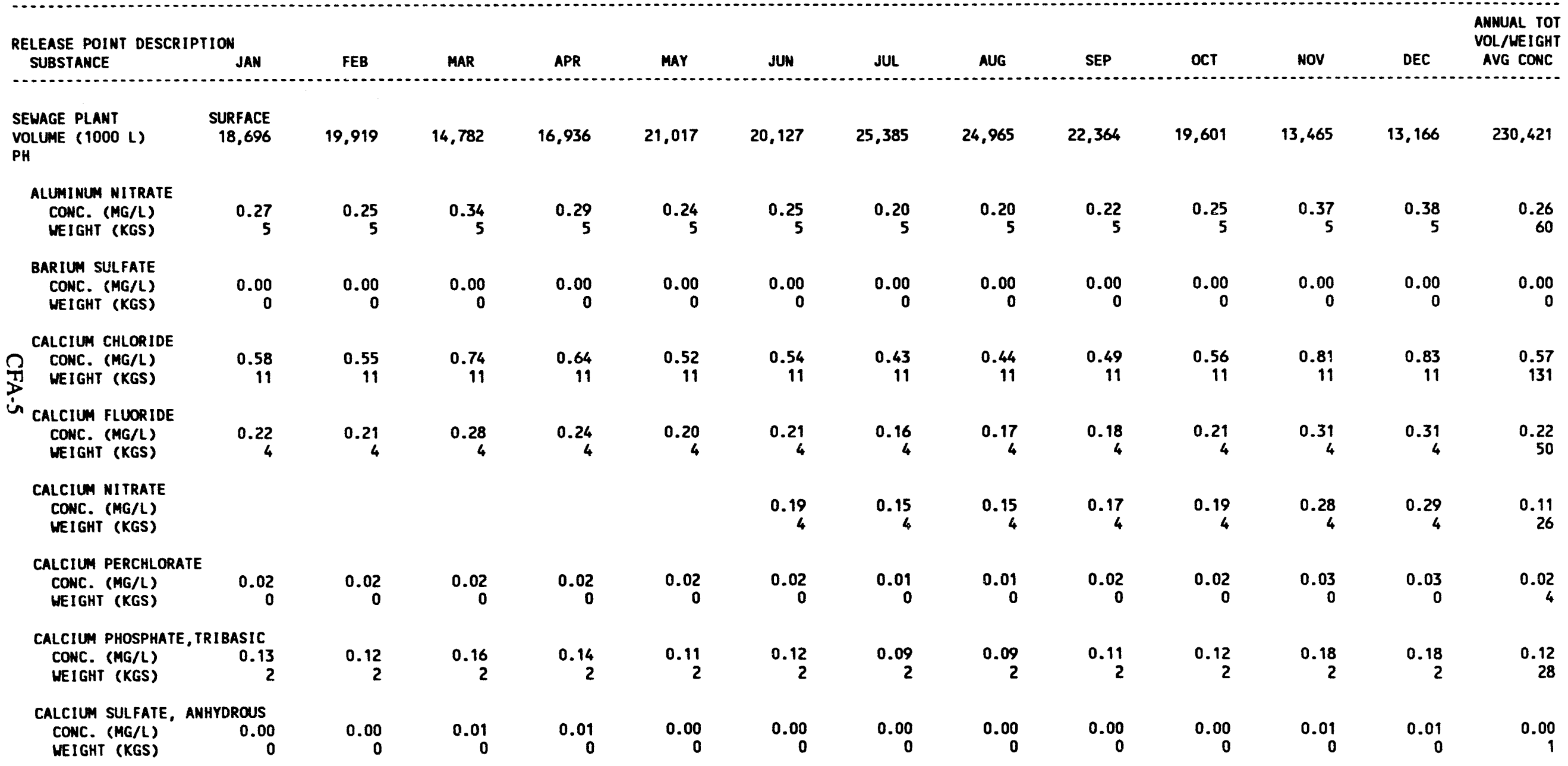

DETAILS MAY NOT ADD UP TO TOTALS BECAUSE OF ROUNDING 0 IN A COLUMA INDICATES A TOTAL OF $<0.5$

0.00 IN A COLUMN INDICATES A TOTAL OF $<0.005$ 


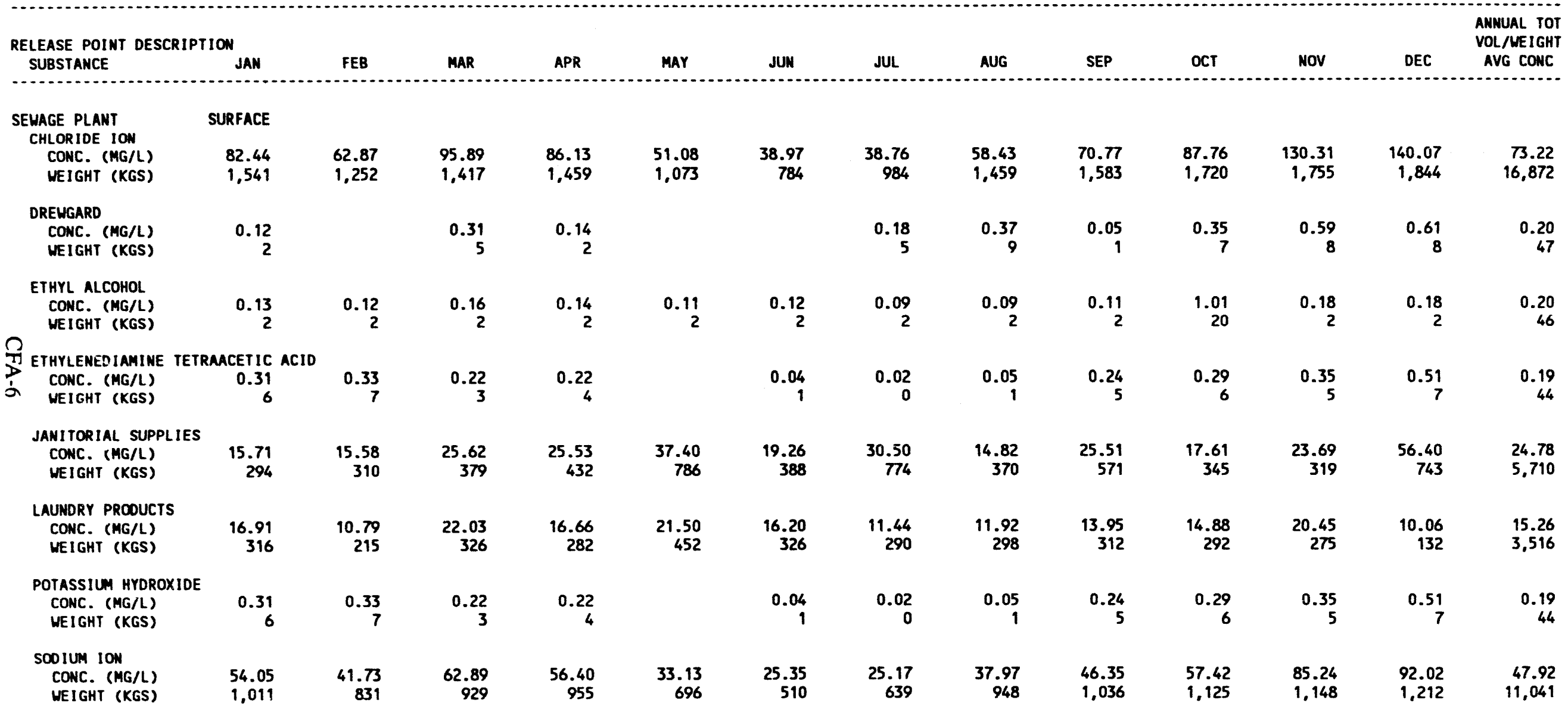




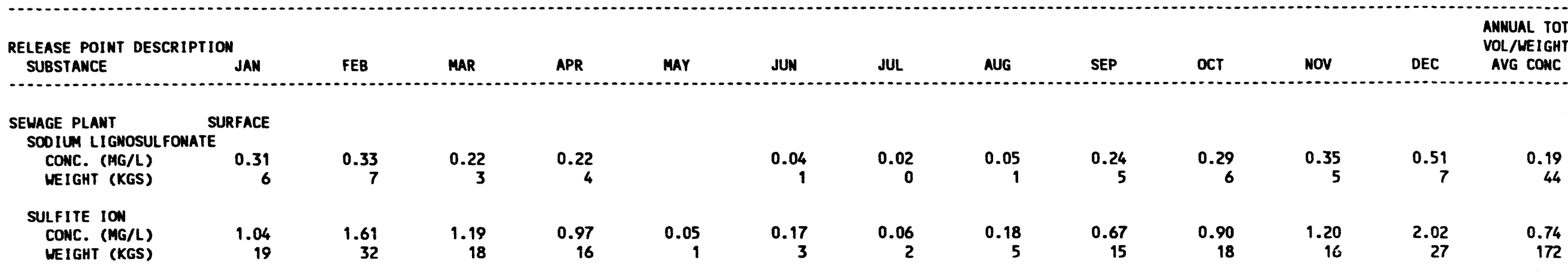


INEL NONRADIOLOGICAL WASTE MANAGEMENT INFORMATION SYSTEM

FOR JANUARY THROUGH DECEMBER 1992

CFA LANDFILL SOLID DISPOSED MASTE SUMMARY

RPT 136-1

(ALL VOLUMES $=$ CUBIC METERS)

\begin{tabular}{|c|c|c|c|c|c|c|c|c|c|c|c|c|c|}
\hline Type of Waste & JAN & FEB & MAR & APR & MAY & JUN & JUL & AUG & SEP & OCT & NOV & DEC & TOTALS \\
\hline ASBESTOS & 9.6 & 3.1 & 0.8 & 0.4 & & & & 0.8 & 6.1 & 0.4 & 3.8 & & 24.8 \\
\hline ASPHALT & & & 16.1 & 5.4 & 8.4 & & & 3.1 & 1.5 & 48.6 & & & 83.0 \\
\hline CAFETERIA GARBAGE & 959.0 & 159.0 & 168.6 & 139.9 & 185.8 & 118.5 & 133.8 & 136.9 & 159.0 & 179.7 & 142.2 & 136.1 & 1.818 .6 \\
\hline DIRT AND GRAVEL & 2.3 & & 313.5 & 19.9 & 109.3 & 3.1 & 7.6 & 119.7 & 1.9 & 195.4 & 6.5 & 1.5 & 780.7 \\
\hline MASONRY AND CONCRETE & 3.1 & 0.8 & 12.6 & 8.0 & 4.2 & 4.6 & 3.8 & 6.5 & 8.4 & 46.6 & 2.3 & & 100.9 \\
\hline SCRAP METAL & 21.4 & 25.2 & 30.2 & 18.7 & 13.0 & 22.6 & 22.9 & 16.1 & 17.6 & 34.4 & 26.8 & 13.8 & 262.6 \\
\hline TRASH， SWEEPINGS, ETC. & 735.9 & 524.9 & 515.7 & 504.3 & 449.2 & 537.1 & 564.7 & 474.1 & 512.3 & 524.5 & 354.8 & 362.8 & $6,060.2$ \\
\hline WEEDS, GRASS AND TREES & 1.1 & 2.3 & 38.6 & 13.8 & 5.5 & 48.2 & 33.6 & 26.4 & 9.9 & 15.7 & 0.4 & & 195.5 \\
\hline WOOO AND SCRAP LUMBER & 16.8 & 11.9 & 32.5 & 16.1 & 29.4 & 20.3 & 32.5 & 7.6 & 44.3 & 33.3 & 29.4 & 11.5 & 285.6 \\
\hline .5 BALLATS; 3 OILY MATE & & & 2.7 & & & & & & & & & & 2.7 \\
\hline 5 BAGS COLOR CODED CLEA & & & & & & & & & & & & & 0.4 \\
\hline ANIMAL TISSUES, PLASTIC & & & & & & & & & & & 0.4 & & 0.4 \\
\hline CARBON FILTERS & & & & & & & & & & & & 0.4 & 0.4 \\
\hline CHEMICALS & & & & & & & & & 3.1 & & & & 3.1 \\
\hline COLOR COOED CLEAN WASTE & & 0.8 & & & & & & & & & & & 0.8 \\
\hline CYLINDERS & & & & & & & & & & & 0.4 & & 0.4 \\
\hline FINGERPRINT KITS & 0.4 & & & & & & & & & & & & 0.4 \\
\hline FLOURESCENT LIGHTS CRUS & & & & & & & & & & & & 0.8 & 0.8 \\
\hline FLOURESCENT TUBES & & & & & & & & 0.8 & & & & & 0.8 \\
\hline GLASS & 3.1 & 6.1 & & & & & & & & & & & 9.2 \\
\hline INSULATORS, METAL, WIRE & 0.4 & & & & & & & & & & & & 0.4 \\
\hline NON-FUSE MUNITIONS & & & & & & & & & & & 0.4 & & 0.4 \\
\hline OUTDATED PHARMACEUTICAL & & & & & 0.2 & & & & & & & & 0.2 \\
\hline PETROLUM PROOUCTS & & & & & & & & & 6.1 & & & & 6.1 \\
\hline PLASTIC & 0.8 & & & & & & & & & & & & 0.8 \\
\hline PLASTIC BUCKETS & & 0.4 & & & & & & & & & & & 0.4 \\
\hline POLY TANK & & & & & & & & & 0.4 & & & & 0.4 \\
\hline RUBBER & & & 0.8 & & & & & & & & & & 0.8 \\
\hline SECURITY KEY'S (BURN) & & & & & & & & 0.4 & & & & & 0.4 \\
\hline USED EXTINGUISHED POWDE & & & & & & 3.1 & & & & & & & 3.1 \\
\hline USED PAINT FILTER & & 0.8 & & & & & & & & & & & 0.8 \\
\hline GRAND TOTALS: & 953.8 & 735.2 & $1,132.4$ & 726.4 & 805.1 & 757.3 & 799.0 & 792.1 & 770.7 & $1,078.5$ & 567.3 & 526.8 & $9,644.7$ \\
\hline
\end{tabular}


PAGE: 1

SCHED. NO. INRPTO1O
IDAHO OPERATIONS OFFICE

UNITED STATES DEPARTMENT OF ENERGY

INEL NONRADIOLOGICAL WASTE MANAGEMENT INFORMATION SYSTEM

FOR JANUARY THROUGH DECEMBER 1992

CFA HATER USAGE AND DISPOSAL SUMMARY

RPT 137-I

CFA

(ALL VOLUMES = NEAREST THOUSAND LITERS)

ANNUAL TO

VOL/WEIGHT

WELL OR BLDG. NO.

JAN

FEB

MAR

APR

MAY

JUN

JUL

SEP

NoV

DEC AVG CONC

\section{WATER DATA}

001 WATER PUMPED (L) 002 WATER PUMPED (L) EBR WATER PUMPED (L)

FS2 WATER PUMPED (L)

MNG HATER PUMPED (L)

PTI WATER PUMPED (L)

$\begin{array}{rr}156 & 7 \\ 24,601 & 22,735 \\ 174 & 159\end{array}$

$\begin{array}{rr}7 & 102 \\ 735 & 315 \\ 159 & 144\end{array}$

MATER PUMPED (L)

$25,529 \quad 23,679$

144
130
105

344
34,708

708
117
182
8

16,001
63,898

63,898

700
170

$\begin{array}{rr}8,805 & 18,829 \\ 50,724 & 68,743\end{array}$

318

113

209

3,488
23

272
207

340

17
336
250

15,785
65,222
12
203
609
28

\begin{tabular}{rr}
285 & 1,613 \\
39,898 & 28,216 \\
249 & 450 \\
52 & 135 \\
441 & 89 \\
24 & 16 \\
\hline
\end{tabular}

84,280

$88,195 \quad 81,859$

40,950

30,520

21,306

182

WATER DISPOSED

$$
\text { AIR (L) }
$$

SURFACE (L)

SUBSURFACE (L)

$\begin{array}{lllr}303 & 144 & 842 & 1,812 \\ 356 & 232 & 258 & 289\end{array}$

28,852
3,084

$\begin{array}{rr}23,640 & 42,926 \\ 463 & 2,259\end{array}$

39.785

$\begin{array}{lll}6,954 & 511 & 403 \\ 2,305 & 975 & 425\end{array}$

977

$233 \quad 521,041$

SEMAGE DATA

18,340

B IOCHEMICAL OXYGEN DEMAND (BCD)

RAW CONC. (MG/L)

$192.0 \quad 195.0 \quad 108.8$

$4,525 \quad 16,648$

17,931

19,665

$23,125 \quad 22,251$

20,059

18,624

13,037

$12,613 \quad 216,506$

X REMOVED

$89.8 \quad 89.8$

118.8

$\begin{array}{llll}131.7 & 136.3 & 112.5 & 122.5\end{array}$

$96.3 \quad 66.3$

66.3
11.3

$\begin{array}{lll}93.8 & 107.5 & 123.5\end{array}$

DISSOLVED OXYGEN (DO)

RAW CONC. (MG/L)

FINAL CONC. (MG/L)

$2.2 \quad 2.9$

$\begin{array}{ll}87.6 & 90.1\end{array}$

$16.3 \quad 12.8$

15.8

23.5

11.0
88.6

83.0

$85.3 \quad 89.5 \quad 87.8$

INCREASED FACTOR

2.4

$\begin{array}{lll}2.9 & 2.4 & 1.5 \\ 7.2 & 6.6 & 6.1\end{array}$

12

1.

6.

SETTLEABLE SOLIDS (SS)

RAW CONC. (ML/L)

7.7

$1.5 \quad 1.8$

3.1

2.9

4.0
3.4

3.4

3.1

6.

1.6

FINAL CONC.

$7.7 \quad 7.1$

$7.1 \quad 5.9$

× REMOVED

100.0

99.9

6.5
0.01

0.018 .5

6.87.

$7.1 \quad 7.7$

$\begin{array}{rr}7.7 & 7.5 \\ 0.01 & 0.01\end{array}$

7.5
0.01
90.9

0.02

99.7

$\begin{array}{lll}9.7 & 6.0 & 7.3 \\ 0.02 & 0.01 & 0.01\end{array}$

7.9

7.7

7.8

7.9

7.9

8.0

7.9

$\begin{array}{ll}8.1 & 7.8\end{array}$

99.9

DETAILS MAY NOT ADD UP TO TOTALS BECAUSE OF ROUNDING

0 IN A COLUMN INDICATES A TOTAL OF $<0.5$

0.00 IN A COLUMN INDICATES A TOTAL OF $<0.005$ 
PAGE: 2

SCHED. NO. INRPTO1O

IDAHO OPERATIONS OFFICE

19-JUN-93

INEL NONRADIOLOGICAL WASTE MANAGEMENT INFORMATION SYSTEM

FOR JANUARY THROUGH DECEMBER 1992

CFA WATER USAGE AND DISPOSAL SUMMARY

RPT 137-I

(ALL VOLUMES = NEAREST THOUSAND LITERS

CFA

\begin{tabular}{|c|c|c|c|c|c|c|c|c|c|c|c|c|c|}
\hline $\begin{array}{l}\text { WELL OR BLDG. NO.I } \\
\text { SEWAGE BLDG. NO. }\end{array}$ & JAN & FEB & MAR & APR & MAY & JUN & JUL & AUG & SEP & OCT & NOV & DEC & $\begin{array}{l}\text { ANNUAL TOT } \\
\text { VOL/WEIGHT } \\
\text { AVG CONC }\end{array}$ \\
\hline TOTAL SEWAGE(L) & 18,340 & 19,688 & 14,525 & 16,648 & 17,931 & 19,665 & 23,125 & 22,251 & 20,059 & 18,624 & 13,037 & 12,613 & 216,506 \\
\hline TOTAL WATER DISPOSED(L) & 18,999 & 20,064 & 15,625 & 18,749 & 49,868 & 43,768 & 68,310 & 64,748 & 29,318 & 20,111 & 13,865 & 13,850 & 377,274 \\
\hline ACCOUNTABILITY (\%) & 74.4 & 84.7 & 1717.4 & 52.7 & 59.2 & 72.5 & 77.5 & 79.1 & 71.6 & 65.9 & 63.1 & 50.9 & 72.4 \\
\hline
\end{tabular}


FOR JANUARY THROUGH DECEMBER 1992

CFA FUEL OIL USAGE AND STACK EFFLUENTS SUMAARY

(ALL VALUES = NEAREST THOUSAND)

CFA

FUEL OIL TYPE
EFFLUENTS

TYPE 2

VOLUME (LITERS)

SO2 (KGS)

NOX (KGS)

6

PARTICULATES (KGS)

DIESEL

VOLUME (LITERS)

SO2 (KGS)

PARTICULATES (KGS)

GRAMD TOTALS:

VOLUME (LITERS)

SO2 (KGS)

NOX (KGS)

PARTICULATES (KGS)

8
0

3
0

2
0

$\begin{array}{ll}33 & 9 \\ 0 & 0\end{array}$

23

16
0

7
0

9
0

58
0

$\begin{array}{rrr}39 & 50 & 402 \\ 0 & 0 & 2\end{array}$


FEB

MAR

APR

MaY

JUN

JUL

AUG

SEP

oct

nov

DEC YTD

BLDG 606

Gross Volumes: $3.79 \mathrm{E}-02$

Gross Weights: $6.35 \mathrm{E}+03$

BLDG 612

Gross Volumes:

Gross Weights:

BLDG 617

Gross Volumes:

Gross Weights:

BLDG 618

Gross Volumes:

Gross Weights:

BLDG 622

Gross Volumes:

BLDG 633

7) GLDG 633 Volumes:

Gross Weights: $8.89 \mathrm{E}+04$

BLDG 689

Gross Volumes:

Gross Weights:

BLDG 690

Gross Volumes:

Gross Weights:

BLDG 691

Gross Volumes: $2.08 E-01$

Gross Weights: $3.40 E+05$

BLDG 698

Gross Volumes:

Gross Weights:

BLDG 699

Gross Volumes:

Gross Weights:

BLDG WED

Gross Volumes:

Gross Weights:

TOTAL VOLUMES: $\quad 3.97 E-01$

TOTAL WEIGHTS: $4.35 \mathrm{E}+05$

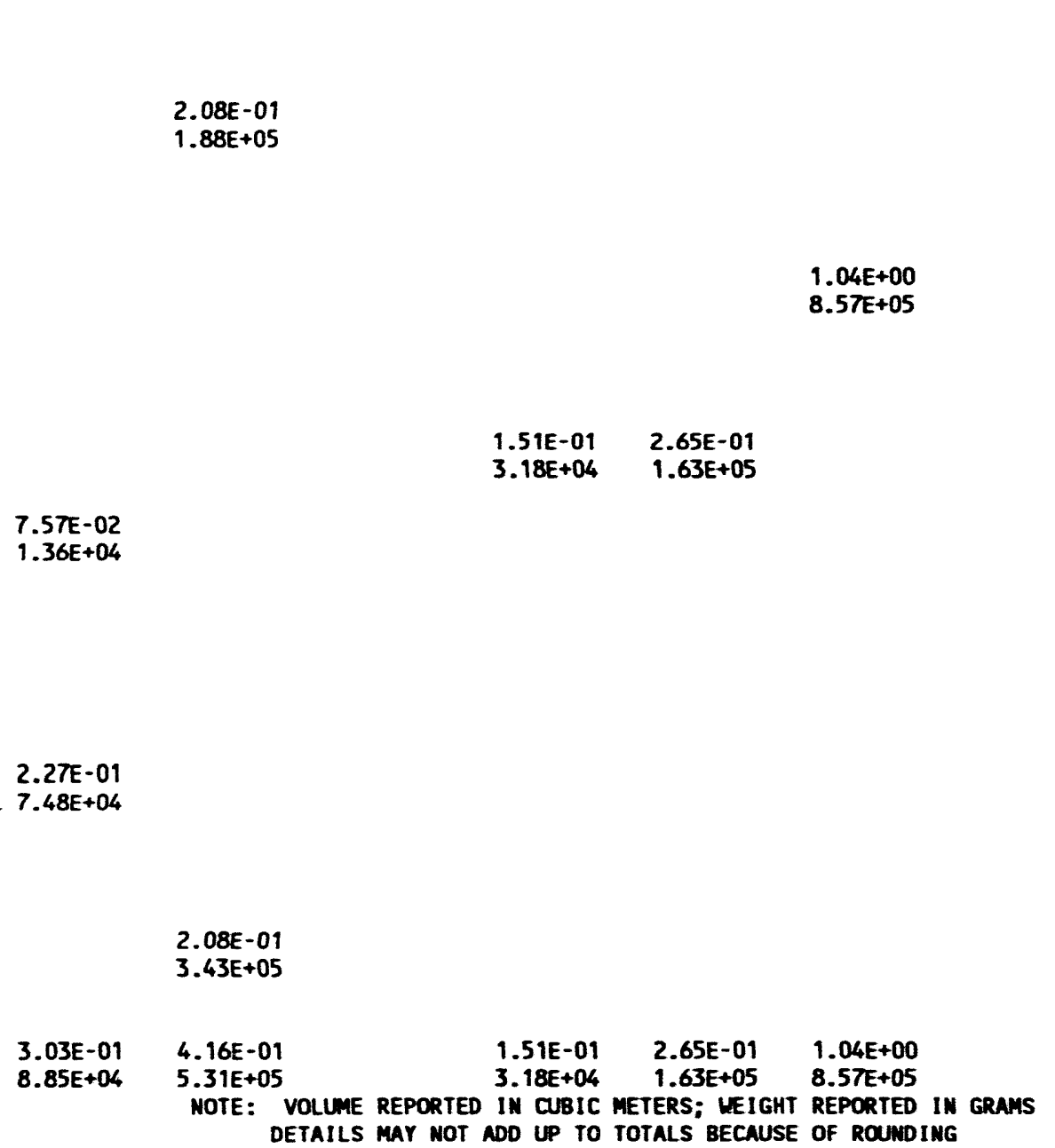
DETAILS MAY NOT ADD UP TO TOTALS BECAUSE OF ROUNDING
3.79E-02

$6.35 E+03$

2.08E-01

$1.88 E+05$

1.51E-01

$9.30 E+04$

$1.04 E+00$ $8.57 \mathrm{E}+05$

4.62E-02 4.62E-02 4.54E+03 4.54E+03

2.12E-01 7.80E-01 $2.36 E+05 \quad 5.20 E+05$

$7.57 E-02$ $1.36 E+04$

1. $14 E-01$

1. $14 \mathrm{E}-01$

3. $19 E+04$

2.08E- 01 $3.40 E+05$

2.27E-01 $7.48 E+04$

2.08E-01

$2.08 E-01$

$1.79 E+05$

$1.79 E+05$

2.08E-01 $3.43 E+05$

1.51E-01 $1.51 E-01$
$9.30 E+04$

1.14E-01

$1.14 E-01$
$3.19 E+04$

4.66E- 01

$4.19 E+05$

$3.31 E+00$ 
Page: 1

Sched. No. INRPT013A
IDAHO OPERATIONS OFFICE

UNITED STATES DEPARTHENT OF ENERGY

INEL NONRADIOLOGICAL MASTE MAMAGEMEMT INFORMATION SYSTEM

MIXED WASTE GENERATED BY CFA

FOR January - Decenber 1992
19- JUN-93

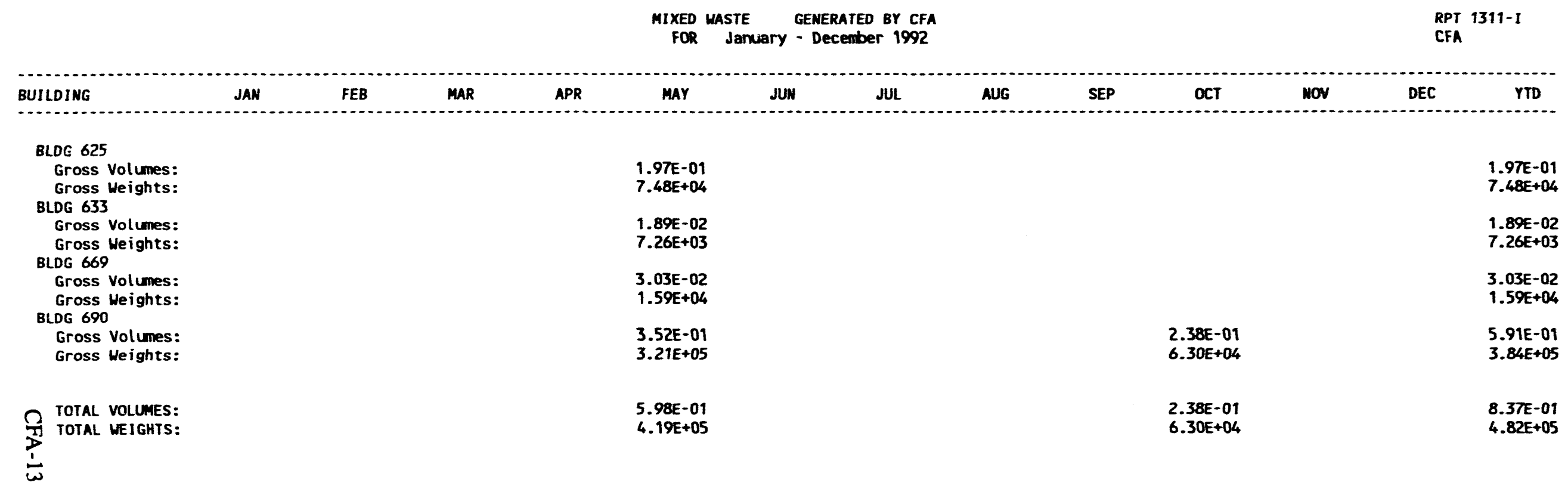


CHEMICAL PROCESSING PLANT, 1992 DETAIL DATA

Report 144 CPP Airborne Disposed Substances Summary $\ldots \ldots \ldots \ldots \ldots$ CPP-3

Report 144 CPP Airborne Release Point And Disposed Substances

Summary $\ldots \ldots \ldots \ldots \ldots \ldots \ldots \ldots \ldots \ldots \ldots \ldots \ldots \ldots \ldots \ldots . \ldots \ldots$

Report 145-CPP Liquid Disposed Substances Summary $\ldots \ldots \ldots \ldots \ldots$ CPP-5

Report 145-CPP Release Point And Liquid Disposed Substances Summary . . . CPP-6

Report 146—CPP Landfill Solid Disposed Waste Summary $\ldots \ldots \ldots \ldots \ldots$ CPP-8

Report 147-CPP Water Usage And Disposal Summary

For January Through December $1992 \ldots \ldots \ldots \ldots \ldots \ldots \ldots \ldots \ldots \ldots$ CPP-9

Report 148-CPP Fuel Oil Usage And Stack Effluents Summary ........ CPP-11

Report 8-INEL Coal Summary For January Through December 1991 . . . . . CPP-12

Report 1410 - Hazardous Waste Generated by CPP For January-

December 1992

CPP-13

Report 1411-Mixed Waste Generated By CPP For January-December 1992 . . CPP-14 
PAGE : 1

SCHED. NO. INRPTOOTA
IDAHO OPERATIONS OFFICE

UNITED STATES DEPARTMENT OF EMERG

INEL NOWRADIOLOGICAL MASTE MAMAGEMENT IMFORWATION SYSTEM

FOR JAMUARY THROUGH DECEMBER 1992

CPP AIRBORME DISPOSED SUBSTAMCES SUMHARY
$25-14=-93$

CPP

\section{anmuin}

AMmuAL

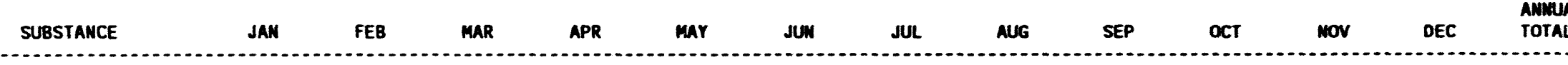

\section{NITRIC OXIDE}

WEIGHT (KGS)

NITROGEN DIOXIDE VEIGHT (KGS)

4,899

1,982

6,881 
PAGE: 1

SCHED. NO. INRPTOO7
IDAHO OPERATIONS OFFICE

UNITED STATES DEPARTMENT OF EMERGY

OLOGICAL WASTE MANAGEMENT IMFORMATION SYSTEM

IMEL NONRADIOLOGICAL WASTE MANAGEMENT IMFORMATI
FOR JAMUARY THROUGH DECEMBER 1992

CPP AIRBORNE RELEASE POINT AND DISPOSED SUBSTANCES SUMMARY

RPT 144-I

CPP

\begin{tabular}{|c|c|c|c|c|c|c|c|c|c|c|c|c|c|}
\hline $\begin{array}{l}\text { RELEASE POINT DESC } \\
\text { SUBSTANCE }\end{array}$ & $\begin{array}{l}\text { IPTION } \\
\text { JAN }\end{array}$ & FEB & MAR & APR & mar & JUN & JUL & AUG & SEP & OCT & NOV & DEC & $\begin{array}{l}\text { ANNUAL TOT } \\
\text { VOL/NEIGHT } \\
\text { AVG CONC }\end{array}$ \\
\hline $\begin{array}{l}\text { STACK } 708 \\
\text { VOLUME (1000 cm) }\end{array}$ & 124,000 & 51 & 124,000 & 105,000 & 79,400 & 300 & 77,900 & 78,400 & 95,000 & 125,000 & 121,000 & 125,000 & $1,055,051$ \\
\hline $\begin{array}{l}\text { NITRIC OXIDE } \\
\text { CONC. (CC/CM) } \\
\text { WEIGHT (KGS) }\end{array}$ & $\begin{array}{r}3.94 \\
544\end{array}$ & & & & & & & & & & $\begin{array}{r}1.64 \\
220\end{array}$ & & $\begin{array}{r}0.65 \\
765\end{array}$ \\
\hline $\begin{array}{l}\text { NITROGEN DIOXIDE } \\
\text { CONC. (CC/CM) } \\
\text { WEIGHT (KGS) }\end{array}$ & $\begin{array}{l}23.13 \\
4,899\end{array}$ & & & & & & & & & & $\begin{array}{r}9.59 \\
1,982\end{array}$ & & $\begin{array}{r}3.82 \\
6,881\end{array}$ \\
\hline
\end{tabular}




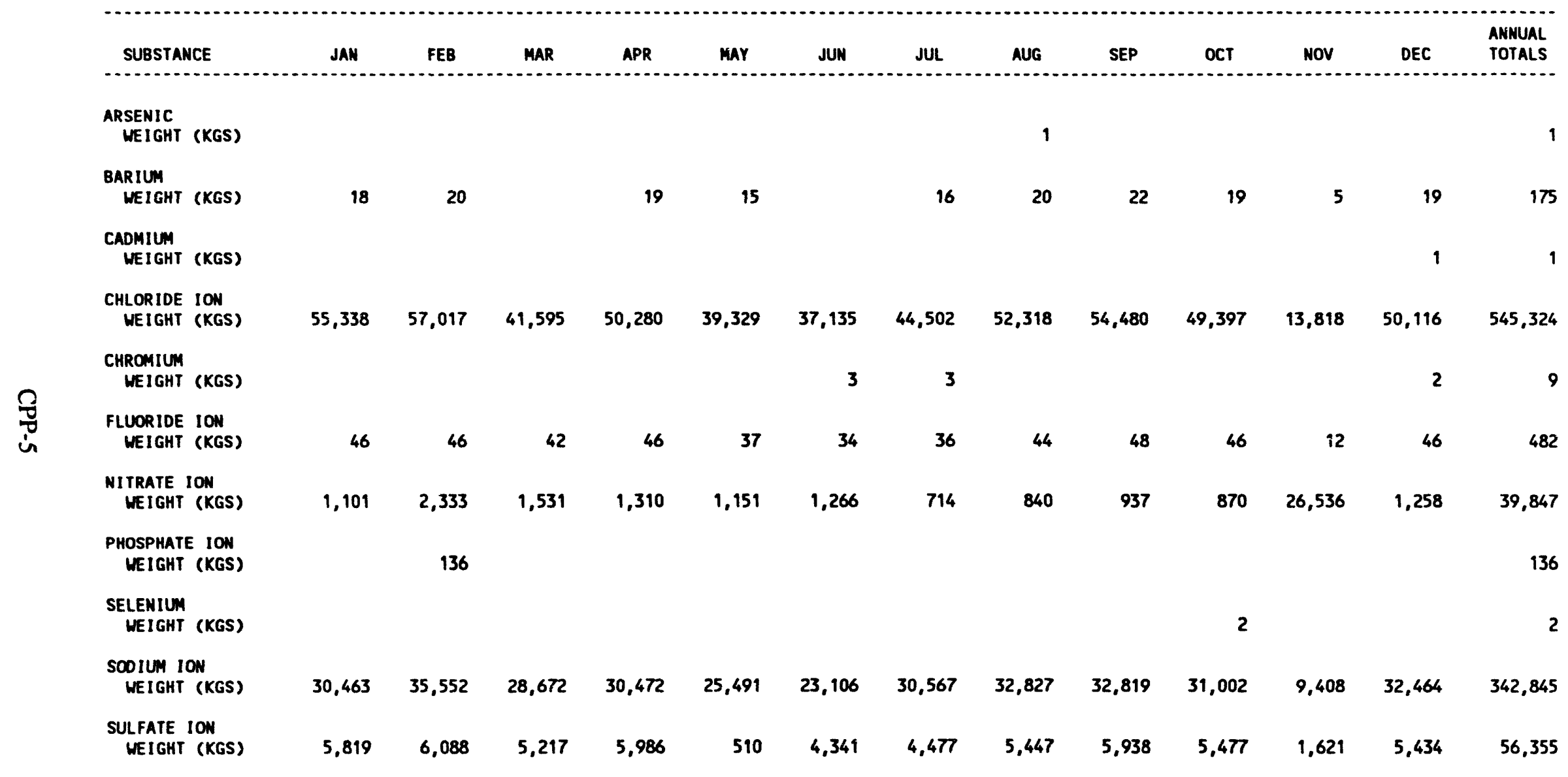


PAGE: 1

SCHED. NO. INRPTOOS
IDAHO OPERATIONS OFFICE

UNITED STATES DEPARTMENT OF EMERGY

INEL NONRADIOLOGICAL UASTE MAMAGEMENT INFORMATION SYSTEM

FOR JANUARY THROUGH DECEMBER 1992

CPP RELEASE POINT AND LIOUID DISPOSED SUBSTAMCES SUMMARY

RPT 145-

RPT 1

\begin{tabular}{|c|c|c|c|c|c|c|c|c|c|c|c|c|c|}
\hline $\begin{array}{l}\text { RELEASE POINT DESCR } \\
\text { SUBSTANCE }\end{array}$ & ${ }_{\text {JION }}^{\text {TAN }}$ & FEB & MAR & APR & mar & JUN & JUL & AUG & SEP & OCT & NOV & DEC & $\begin{array}{l}\text { ANNUAL TOT } \\
\text { VOL/NEIGHT } \\
\text { AVG CONC }\end{array}$ \\
\hline $\begin{array}{l}\text { S PERCOLATION POND } \\
\text { VOLUME }(1000 \mathrm{~L}) \\
\text { PH }\end{array}$ & $\begin{array}{l}\text { SURFACE } \\
210,090\end{array}$ & 222,203 & 199,112 & 217,651 & 182,078 & 165,043 & 179,807 & 205,69 & 218,796 & 206,683 & 58,800 & 202,897 & $2,268,338$ \\
\hline $\begin{array}{l}\text { ARSENIC } \\
\text { CONC. (MG/L) } \\
\text { WEIGHT (KGS) }\end{array}$ & & & & & & & & $\begin{array}{r}0.00 \\
1\end{array}$ & & & & & $\begin{array}{r}0.00 \\
1\end{array}$ \\
\hline $\begin{array}{l}\text { BARIUM } \\
\text { CONC. (MG/L) } \\
\text { WEIGHT (KGS) }\end{array}$ & $\begin{array}{r}0.09 \\
18\end{array}$ & $\begin{array}{r}0.09 \\
20\end{array}$ & & $\begin{array}{r}0.09 \\
19\end{array}$ & $\begin{array}{r}0.08 \\
15\end{array}$ & & $\begin{array}{r}0.09 \\
16\end{array}$ & $\begin{array}{r}0.10 \\
20\end{array}$ & $\begin{array}{r}0.10 \\
22\end{array}$ & $\begin{array}{r}0.09 \\
19\end{array}$ & $\begin{array}{r}0.09 \\
5\end{array}$ & $\begin{array}{r}0.10 \\
19\end{array}$ & $\begin{array}{r}0.08 \\
175\end{array}$ \\
\hline $\begin{array}{l}\text { CADMIUM } \\
\text { CONC. (MG/L) } \\
\text { MEIGHT (KGS) }\end{array}$ & & & & & & & & & & & & $\begin{array}{r}0.00 \\
1\end{array}$ & $\begin{array}{r}0.00 \\
1\end{array}$ \\
\hline $\begin{array}{l}\text { O CHLORIDE ION } \\
\text { CONC. (MG/L) } \\
\text { WEIGHT (KGS) }\end{array}$ & $\begin{array}{l}263.40 \\
55,338\end{array}$ & $\begin{array}{l}256.60 \\
57,017\end{array}$ & $\begin{array}{l}208.90 \\
41,595\end{array}$ & $\begin{array}{l}231.00 \\
50,280\end{array}$ & $\begin{array}{l}216.00 \\
39,329\end{array}$ & $\begin{array}{l}225.00 \\
37,135\end{array}$ & $\begin{array}{l}247.50 \\
44,502\end{array}$ & $\begin{array}{l}255.00 \\
52,318\end{array}$ & $\begin{array}{l}249.00 \\
54,480\end{array}$ & $\begin{array}{l}239.00 \\
49,397\end{array}$ & $\begin{array}{l}235.00 \\
13,818\end{array}$ & $\begin{array}{l}247.05 \\
50,116\end{array}$ & $\begin{array}{r}240.41 \\
545,324\end{array}$ \\
\hline $\begin{array}{l}\text { CHRONIUM } \\
\text { CONC. (MG/L) } \\
\text { WEIGHT (KGS) }\end{array}$ & & & & & & $\begin{array}{r}0.02 \\
3\end{array}$ & $\begin{array}{r}0.02 \\
3\end{array}$ & & & & & $\begin{array}{r}0.01 \\
2\end{array}$ & $\begin{array}{r}0.00 \\
9\end{array}$ \\
\hline $\begin{array}{l}\text { FLUORIDE ION } \\
\text { CONC. (MG/L) } \\
\text { WEIGHT (KGS) }\end{array}$ & $\begin{array}{r}0.22 \\
46\end{array}$ & $\begin{array}{r}0.21 \\
46\end{array}$ & $\begin{array}{r}0.21 \\
42\end{array}$ & $\begin{array}{r}0.21 \\
46\end{array}$ & $\begin{array}{r}0.20 \\
37\end{array}$ & $\begin{array}{r}0.21 \\
34\end{array}$ & $\begin{array}{r}0.20 \\
36\end{array}$ & $\begin{array}{r}0.22 \\
44\end{array}$ & $\begin{array}{r}0.22 \\
48\end{array}$ & $\begin{array}{r}0.22 \\
46\end{array}$ & $\begin{array}{r}0.21 \\
12\end{array}$ & $\begin{array}{r}0.23 \\
46\end{array}$ & $\begin{array}{r}0.21 \\
482\end{array}$ \\
\hline $\begin{array}{l}\text { NITRATE ION } \\
\text { CONC. (MG/L) } \\
\text { WEIGHT (KGS) }\end{array}$ & $\begin{array}{r}5.24 \\
1,101\end{array}$ & $\begin{array}{l}10.50 \\
2,333\end{array}$ & $\begin{array}{r}7.69 \\
1.531\end{array}$ & $\begin{array}{r}6.02 \\
1,310\end{array}$ & $\begin{array}{r}6.32 \\
1.151\end{array}$ & $\begin{array}{r}7.67 \\
1.266\end{array}$ & $\begin{array}{r}3.97 \\
714\end{array}$ & $\begin{array}{r}4.10 \\
840\end{array}$ & $\begin{array}{r}4.28 \\
937\end{array}$ & $\begin{array}{r}4.21 \\
870\end{array}$ & $\begin{array}{l}451.30 \\
26,536\end{array}$ & $\begin{array}{r}6.20 \\
1,258\end{array}$ & $\begin{array}{r}17.57 \\
39,847\end{array}$ \\
\hline $\begin{array}{l}\text { PHOSPHATE IOW } \\
\text { CONC. (MG/L) } \\
\text { WEIGHT (KGS) }\end{array}$ & & $\begin{array}{r}0.61 \\
136\end{array}$ & & & & & & & & & & & $\begin{array}{r}0.06 \\
136\end{array}$ \\
\hline
\end{tabular}

DETAILS MAY NOT ADD UP TO TOTALS BECAUSE OF ROUNDING

0 IN A COLUMN IMDICATES A TOTAL OF $<0.5$

0.00 IN $\hat{A}$ COLUAN INDICATES $\hat{A}$ TOTAL OF $<0.005$ 
PAGE: 2

SCHED. NO. INRPTOOS
IDAHO OPERATIONS OFFICE

UNITED STATES DEPARTMENT OF ENERGY

INEL NONRADIOLOGICAL WASTE MANAGEMENT INFORMATION SYSTEM

FOR JAMUARY THROUGH DECEMBER 1992

CPP RELEASE POINT AND LIQUID DISPOSED SUBSTANCES SUMMARY
25- JUN-93

RPT 145-1

RPP

\begin{tabular}{|c|c|c|c|c|c|c|c|c|c|c|c|c|c|}
\hline $\begin{array}{l}\text { RELEASE POINT DESCR I } \\
\text { SUBSTANCE }\end{array}$ & TION $_{\text {JAN }}$ & FEB & MAR & APR & MAY & JUN & JUL & AUG & SEP & OCT & NOV & DEC & $\begin{array}{l}\text { ANNUAL TOT } \\
\text { VOL/WEIGHT } \\
\text { AVG CONC }\end{array}$ \\
\hline $\begin{array}{l}\text { S PERCOLATION POND } \\
\text { SELENIUM } \\
\text { CONC. (MG/L) } \\
\text { WEIGHT (KGS) }\end{array}$ & SURFACE & & & & & & & & & $\begin{array}{r}0.01 \\
2\end{array}$ & & & $\begin{array}{r}0.00 \\
2\end{array}$ \\
\hline $\begin{array}{l}\text { SOOIUN ION } \\
\text { CONC. (MG/L) } \\
\text { WEIGHT (KGS) }\end{array}$ & $\begin{array}{l}145.00 \\
30,463\end{array}$ & $\begin{array}{l}160.00 \\
35,552\end{array}$ & $\begin{array}{l}144.00 \\
28,672\end{array}$ & $\begin{array}{l}140.00 \\
30,472\end{array}$ & $\begin{array}{l}140.00 \\
25,491\end{array}$ & $\begin{array}{l}140.00 \\
23,106\end{array}$ & $\begin{array}{l}170.00 \\
30,567\end{array}$ & $\begin{array}{l}160.00 \\
32,827\end{array}$ & $\begin{array}{l}150.00 \\
32,819\end{array}$ & $\begin{array}{l}150.00 \\
31,002\end{array}$ & $\begin{array}{r}160.00 \\
9.408\end{array}$ & $\begin{array}{l}16 n .00 \\
32.464\end{array}$ & $\begin{array}{r}151.14 \\
342,845\end{array}$ \\
\hline $\begin{array}{l}\text { SULFATE ION } \\
\text { CONC. (MG/L) } \\
\text { WEIGHT (KGS) }\end{array}$ & $\begin{array}{l}27.70 \\
5,819\end{array}$ & $\begin{array}{l}27.40 \\
6.088\end{array}$ & $\begin{array}{l}26.20 \\
5.217\end{array}$ & $\begin{array}{l}27.50 \\
5,986\end{array}$ & $\begin{array}{r}2.80 \\
510\end{array}$ & $\begin{array}{l}26.30 \\
4,341\end{array}$ & $\begin{array}{l}24.90 \\
4.477\end{array}$ & $\begin{array}{l}26.55 \\
5.447\end{array}$ & $\begin{array}{l}27.14 \\
5,938\end{array}$ & $\begin{array}{l}26.50 \\
5,477\end{array}$ & $\begin{array}{l}27.57 \\
1.621\end{array}$ & $\begin{array}{l}26.78 \\
5,434\end{array}$ & $\begin{array}{r}24.84 \\
56,355\end{array}$ \\
\hline$\stackrel{3}{3}$ & & & & & & & & & & & & & \\
\hline
\end{tabular}

DETAILS MAY NOT ADD UP TO TOTALS BECAUSE OF ROUHDING

0 IN A COLUMN INDICATES A TOTAL OF $<0.5$

0.00 IN A COLUNA INDICATES A TOTAL OF $<0.005$ 
PAGE: 1

IDAHO OPERATIONS OFFICE

19- JUN-93

SCHED. NO. INRPTOO9

UNITED STATES DEPARTMENT OF ENERGY

INEL NONRADIOLOGICAL WASTE MANAGEMENT INFORMATION SYSTEM

FOR JANUARY THROUGH DECEMBER 1992

CPP LANDFILL SOLID DISPOSED MASTE SUMMARY

RPT 146-I

CPP

(ALL VOLUMES = CUBIC METERS)

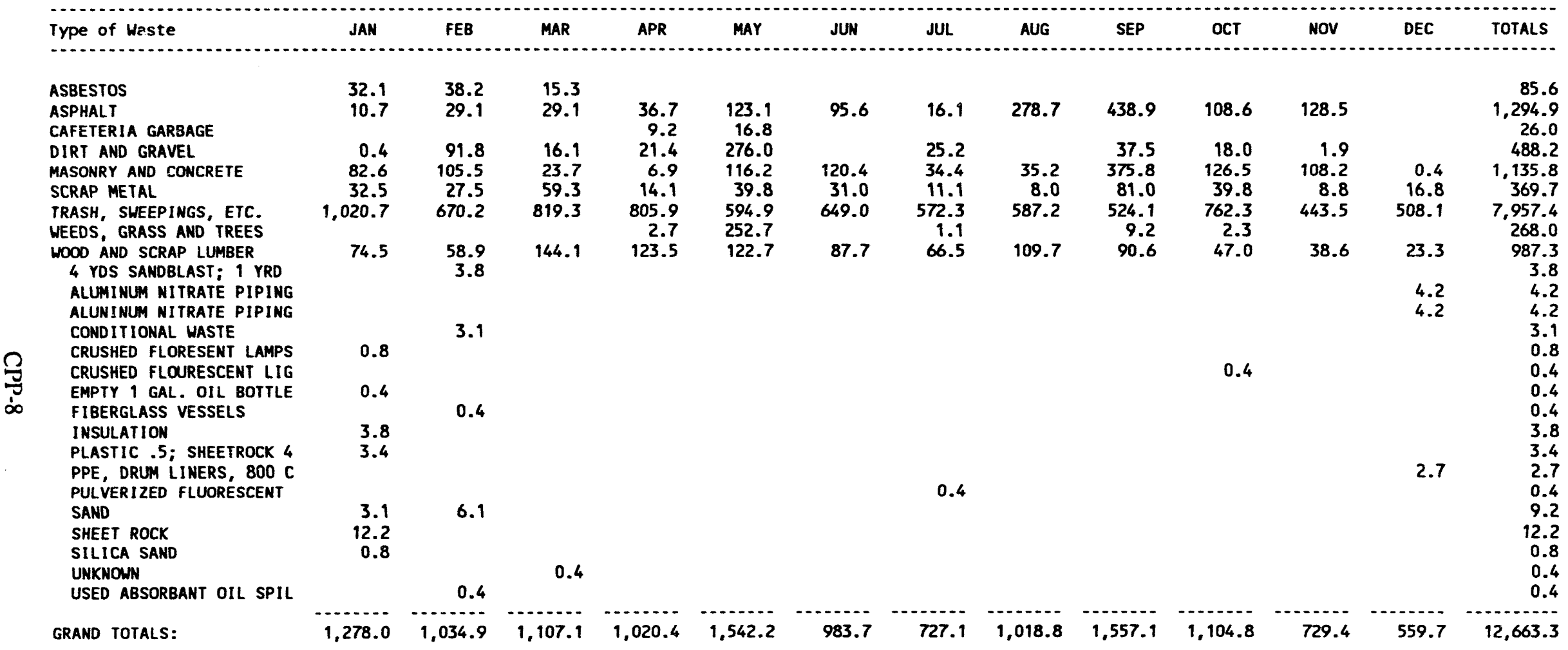


(ALL VOLUMES = NEAREST THOUSAND LITERS)

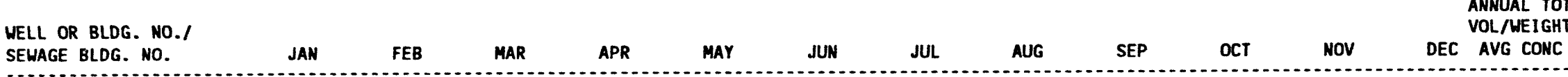

\section{WATER DATA}

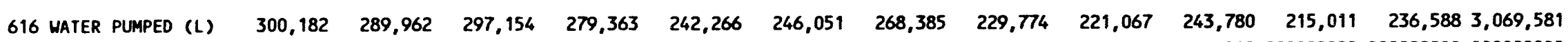

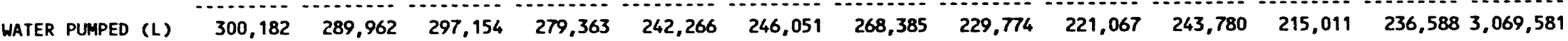

WATER DISPOSED
AIR (L)
SURFACE (L)
$210,090 \quad 222,203$
$99.112 \quad 217$
217,661
$182,078 \quad 165,043$
$179,807 \quad 205,169 \quad 218,796 \quad 206,683$
$222,582 \quad 202,8972,432,120$

SEWAGE DATA

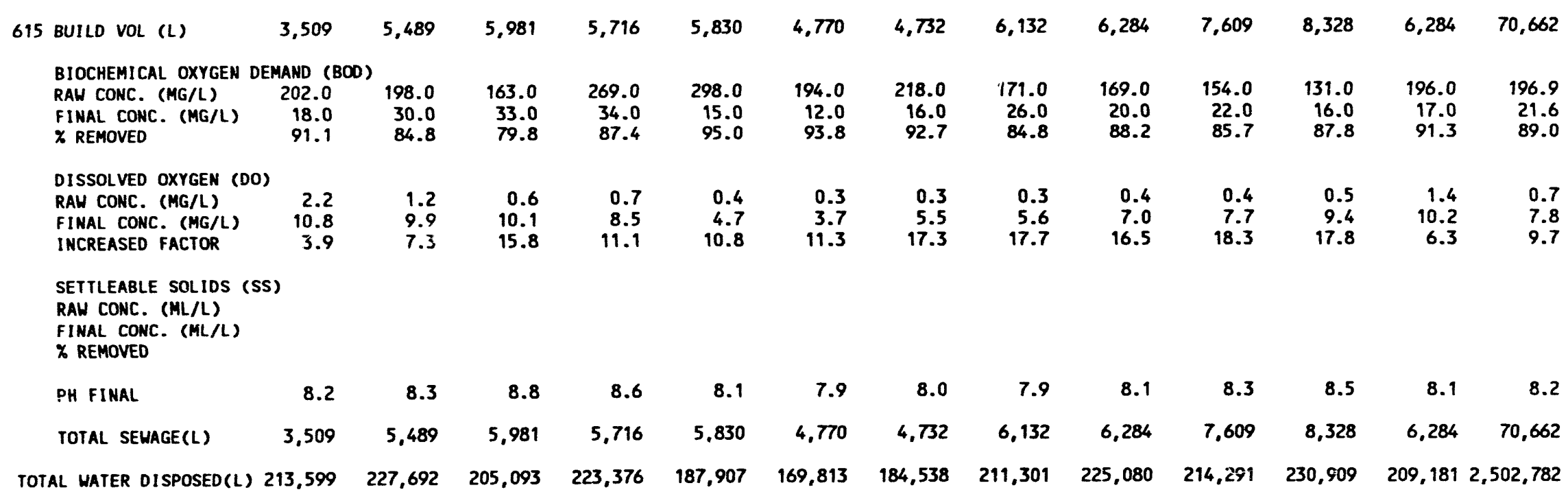

DETAILS MAY NOT ADD UP TO TOTALS BECAUSE OF ROUNDING

0 IH A COLUMN INDICATES A TOTAL OF $<0.5$

0.0 IN A COLUMA INDICATES A TOTAL OF $<0.05$

0.00 IN A COLUAN INDICATES A TOTAL OF $<0.005$ 
PAGE: 2

SCHED. NO. INRPT010

IDAHO OPERATIONS OFFICE

19-JUN-93

INEL NONRADIOLOGICAL STATES DEPARTMENT OF ENERGY

IOLOGICAL MASTE MANAGEMENT INFORMATION SYSTEM

FOR JAMUARY THROUGH DECEMBER 1992

CPP MATER USAGE AND DISPOSAL SUMMARY

RPT 147-1

(ALL VOLUMES = NEAREST THOUSAND LITERS)

CPP

ANMUAL TOT

VOL/WEIGHT

WELL OR BLDG. NO./

SEWAGE BLDG. NO.

FEB MAR APR

MAY

AUG

SEP

OCT

NOV DEC AVG CONC

ACCOUNTABILITY $(\boldsymbol{x})$

71.2

69.0

80.0

77.6

69.0

68.8

92.0

101.8

87.9

107.4

88.4

81.5

0.00 IN A COLUMN INDICATES A TOTAL OF $<0.005$ 
FOR JANUARY THROUGH DECEMBER 1992

CPP FUEL OIL USAGE AND STACK EFFLUENTS SUMUARY

(ALL VALUES = MEAREST THOUSAND)

\begin{tabular}{|c|c|c|c|c|c|c|c|c|c|c|c|c|c|}
\hline $\begin{array}{l}\text { FUEL OIL TYPE } \\
\text { EFFLUENTS }\end{array}$ & JAN & FEB & MAR & APR & MAY & JUN & JUL & AUG & SEP & OCT & NOV & DEC & $\begin{array}{l}\text { ANNUAL } \\
\text { TOTALS }\end{array}$ \\
\hline $\begin{array}{l}\text { TYPE } 2 \\
\text { VOLUME (LITERS) } \\
\text { SOL (KGS) } \\
\text { NOX (KGS) } \\
\text { PARTICULATES (KGS) }\end{array}$ & $\begin{array}{r}32 \\
1\end{array}$ & $\begin{array}{r}16 \\
0\end{array}$ & $\begin{array}{r}11 \\
0\end{array}$ & $\begin{array}{l}8 \\
0\end{array}$ & $\begin{array}{r}62 \\
1\end{array}$ & $\begin{array}{r}98 \\
2\end{array}$ & $\begin{array}{l}6 \\
0\end{array}$ & $\begin{array}{r}30 \\
0\end{array}$ & $\begin{array}{r}30 \\
1\end{array}$ & $\begin{array}{r}104 \\
2\end{array}$ & $\begin{array}{r}163 \\
3\end{array}$ & $\begin{array}{r}151 \\
3\end{array}$ & $\begin{array}{r}713 \\
12\end{array}$ \\
\hline $\begin{array}{l}\text { GRAND TOTALS: } \\
\text { VOLUME (LITERS) } \\
\text { SO2 (KGS) } \\
\text { NOX (KGS) } \\
\text { PARTICULATES (KGS) }\end{array}$ & $\begin{array}{r}32 \\
1\end{array}$ & $\begin{array}{r}16 \\
0\end{array}$ & $\begin{array}{r}11 \\
0\end{array}$ & $\begin{array}{l}8 \\
0\end{array}$ & $\begin{array}{r}62 \\
1\end{array}$ & $\begin{array}{r}98 \\
2\end{array}$ & $\begin{array}{l}6 \\
0\end{array}$ & $\begin{array}{r}30 \\
0\end{array}$ & $\begin{array}{r}30 \\
1\end{array}$ & $\begin{array}{r}104 \\
2\end{array}$ & $\begin{array}{r}163 \\
3\end{array}$ & $\begin{array}{r}151 \\
3\end{array}$ & $\begin{array}{r}713 \\
12\end{array}$ \\
\hline
\end{tabular}


PAGE: 1

SCHED. NO. INRPT020
IDAHO OPERATIONS OFFICE

UNITED STATES DEPARTMENT OF ENERGY

INEL NONRADIOLOGICAL WASTE MANAGEMENT INFORMATION SYSTEM
19-JUN-93

RPT 8-1

FOR JANUARY THROUGH DECEMBER 1992

\begin{tabular}{|c|c|c|c|c|c|c|c|c|c|c|c|c|c|}
\hline AREA & JAN & FEB & MAR & APR & MaY & JUN & JUL & AUG & SEP & OCT & NOV & DEC & TOTALS \\
\hline CPP & & & & & & & & & & & & & \\
\hline $\begin{array}{l}\text { TONS BURNED } \\
\text { AVG BTUS }\end{array}$ & $\begin{array}{r}1,580 \\
.01000\end{array}$ & $\begin{array}{r}1,344 \\
.01000\end{array}$ & $\begin{array}{r}1,320 \\
.01232\end{array}$ & $\begin{array}{r}1,020 \\
.01232\end{array}$ & $\begin{array}{r}790 \\
.01232\end{array}$ & $\begin{array}{r}446 \\
.01232\end{array}$ & $\begin{array}{r}673 \\
.01232\end{array}$ & $\begin{array}{r}632 \\
.01232\end{array}$ & $\begin{array}{r}778 \\
.01293\end{array}$ & $\begin{array}{r}806 \\
.01293\end{array}$ & $\begin{array}{r}1,325 \\
.01309\end{array}$ & $\begin{array}{r}1.750 \\
.01309\end{array}$ & $\begin{array}{l}12,464 \\
.01216\end{array}$ \\
\hline $\begin{array}{l}\text { NO2 (M TONS) } \\
\text { SO2 (M TONS) }\end{array}$ & $\begin{array}{l}6 \\
2\end{array}$ & $\begin{array}{l}4 \\
2\end{array}$ & $\begin{array}{l}5 \\
2\end{array}$ & & $\begin{array}{l}4 \\
2\end{array}$ & $\begin{array}{l}3 \\
1\end{array}$ & & $\begin{array}{l}4 \\
1\end{array}$ & $\begin{array}{l}6 \\
1\end{array}$ & $\begin{array}{l}6 \\
1\end{array}$ & $\begin{array}{l}8 \\
2\end{array}$ & $\begin{array}{r}10 \\
2\end{array}$ & $\begin{array}{l}64 \\
17\end{array}$ \\
\hline $\begin{array}{l}\text { GRAND TOTALS: } \\
\text { TONS BURNED } \\
\text { NO2 (M TONS) } \\
\text { SO2 (M TONS) }\end{array}$ & $\begin{array}{r}1,580 \\
6 \\
2\end{array}$ & $\begin{array}{r}1,344 \\
4 \\
2\end{array}$ & $\begin{array}{r}1,320 \\
5 \\
2\end{array}$ & $\begin{array}{r}1,020 \\
4 \\
2\end{array}$ & $\begin{array}{r}790 \\
4 \\
2\end{array}$ & $\begin{array}{r}446 \\
3 \\
1\end{array}$ & $\begin{array}{r}673 \\
4 \\
1\end{array}$ & $\begin{array}{r}632 \\
4 \\
1\end{array}$ & $\begin{array}{r}778 \\
6 \\
1\end{array}$ & $\begin{array}{r}806 \\
6 \\
1\end{array}$ & $\begin{array}{r}1,325 \\
8 \\
2\end{array}$ & $\begin{array}{r}1,750 \\
10 \\
2\end{array}$ & $\begin{array}{r}12,464 \\
64 \\
17\end{array}$ \\
\hline
\end{tabular}

$\frac{8}{0}$ 


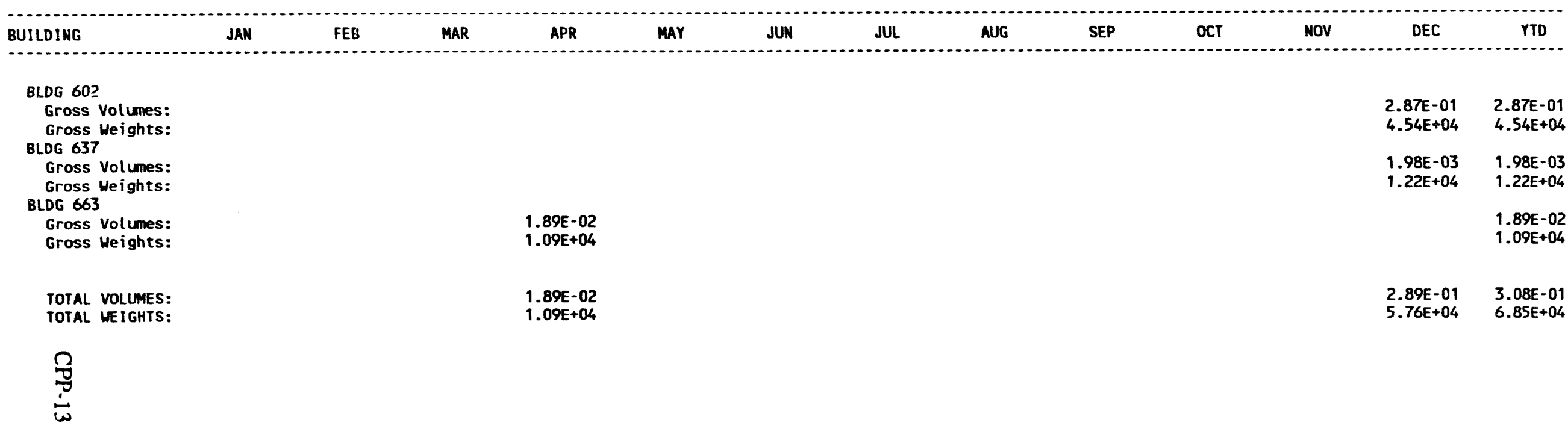


UNEL MONRADIOLOGITED STATES DEPARTMENT OF EMERGY

RPT 1411-I

MIXED MASTE

GENERATED BY CPP

CPP

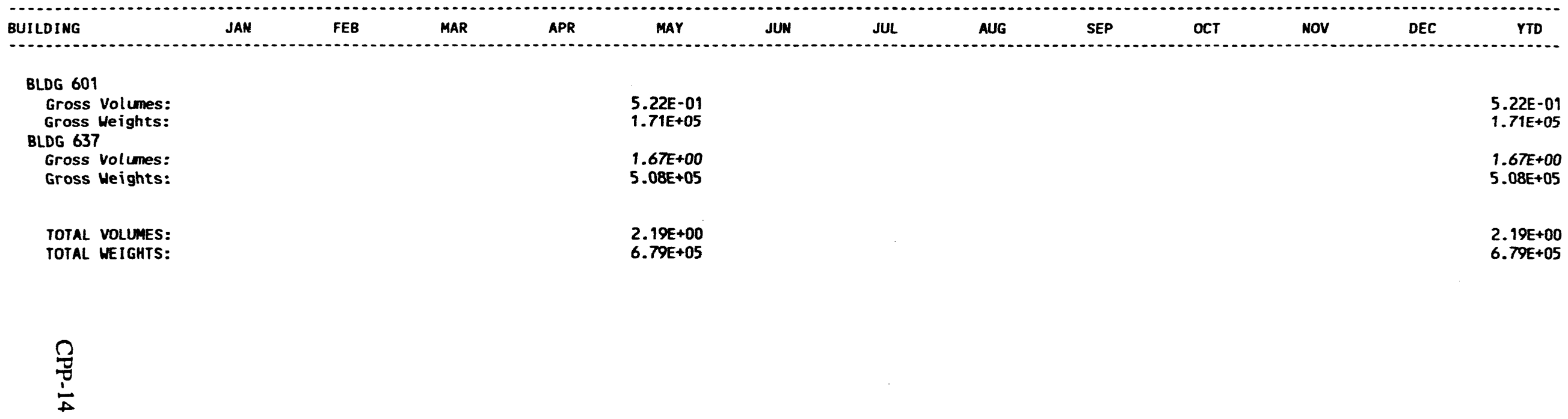


CONTAINMENT TEST FACILITY, 1992 DETAIL DATA

Report 165-CTF Liquid Disposed Substances Summary . . . . . . . . . . CTF-3

Report 165-CTF Release Point And Liquid Disposed Substances Summary . . . C CTF-4

Report 167-CTF Water Usage And Disposal Summary For January

Through December $1992 \ldots \ldots \ldots \ldots \ldots \ldots \ldots \ldots \ldots \ldots \ldots \ldots$ CTF-5 
PAGE: 1

SCHED. NO. INRPTOOBA
IDAHO OPERATIONS OFFICE

UNITED STATES DEPARTMENT OF EMERGY

INEL NONRADIOLOGICAL MASTE MANAGEMENT INFORMATION SYSTEM

FOR JAMUARY THROUGH DECEMBER 1992

CTF LIOUID DISPOSED SUBSTANCES SUMHARY

RPT 165-I

SUBSTANCE

JANITORIAL SUPPLIES

MEIGHT (KGS) 
PAGE: 1

SCHED. NO. INRPTOOS
IDAHO OPERATIONS OFFICE

25- JUN-93

UNITED STATES DEPARTMENT OF ENERGY

INEL NONRADIOLOGICAL MASTE MANAGEMENT INFORMATION SYSTEM

FOR JAMUARY THROUGH DECEMBER 1992

CTF RELEASE POINT AND LIQUID DISPOSED SUBSTAMCES SUMMURY

RPT 165-I

CTF

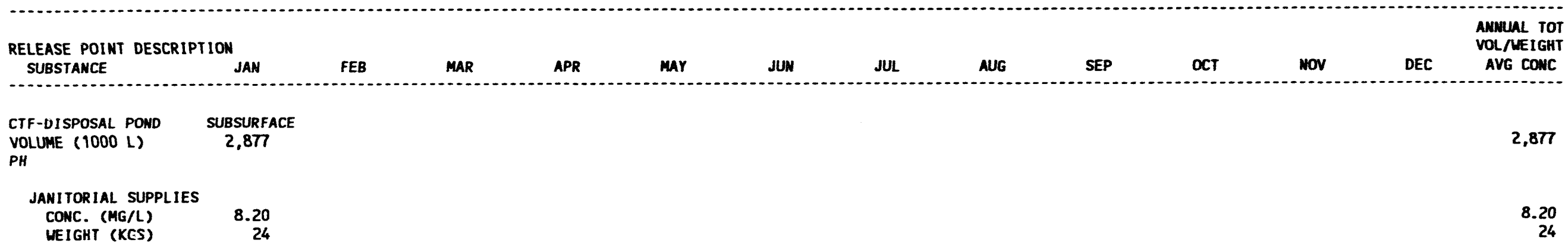

勇

DETAILS MAY NOT ADD UP TO TOTALS BECAUSE OF ROUNDING

O IN A COLUAM INDICATES A TOTAL OF $<0.5$

0.00 IN A COLUPM IMDICATES A TOTAL OF $<0.005$ 
PAGE: 1

SCHED. NO. INRPTO1O

IDAHO OPERATIONS OFFICE

UNITED STATES DEPARTMENT OF EMERGY

RPT 167-I

FOR JANUARY THROUGH DECEMBER 1992

CTF WATER USAGE AND DISPOSAL SUMMARY

CTF

(ALL VOLUMES = NEAREST THOUSAND LITERS)

ANNUAL TOT
VOL/WEIGHT

WELL OR BLDG. NO./

JAN

FEB

MAR

APR

MAY

JUN

JUL

AUG

SEP

OCT

Nov

DEC AVG CONC

WATER DATA

632 MATER PUAPED (L)

639 WATER PUMPED (L)

1,633
1,333
.- .6

, 333

WATER PUMPED (L) $\quad 2,965$

ER DISPOSED

AIR (L)

SURFACE (L)

2,877

SUBSURFACE (L)

877

SEWAGE DATA

736 BUILD VOL (L)

76

76

BIOCHEMICAL OXYGEN DEMAND (BOD)

RAH CONC. (MG/L)

FINAL. CONC. (MG/L)

\% REMOVED

DISSOLVED OXYGEN (DO)

RAW CONC. (MG/L)

FINAL CONC. (MG/L)

INCREASED FACTOR

SETTLEABLE SOLIDS (SS)

RAW CONC. (ML/L)

FINAL CONC. (ML/L)

\% REMOVED

PH FINAL

TOTAL SEWAGE(L)

TOTAL WATER DISPOSED(L) $\quad 2,953$

DETAILS MAY NOT ADD UP TO TOTALS BECAUSE OF ROUMDING

O IN A COLUMN INDICATES A TOTAL OF $<0.5$

0.0 IN A COLLAN INDICATES A TOTAL OF $<0.05$

0.00 IN A COLUMN INDICATES A TOTAL OF $<0.005$ 
PAGE: 2

SCHED. NO. INRPTO1O

IDAHO OPERATIONS OFFICE

UNITED STATES DEPARTMENT OF ENERGY

19- JUN-93

INEL NONRADIOLOGICAL WASTE MANAGEMENT INFORMATION SYSTEM

FOR JANUARY THROUGH DECEMBER 1992

CTF WATER USAGE AND DISPOSAL SUMUARY

RPT 167-I

(ALL VOLUMES = NEAREST THOUSAND LITERS)

\begin{abstract}
NELL OR BLDG. NO./
SEWAGE BLDG. NO.
\end{abstract}

ACCOUNTABILITY (x)

FEB

MAR

APR

\begin{abstract}
maY
\end{abstract}
JUN

JUL

AUG

SEP

oCr

Nov

VOL/WEIGHT DEC AVG CONC

99.6

99.6

0 IN A COLUMN INDICATES A TOTAL OF $<0.5$

0.0 IN A COLLMN IMDICATES $A$ TOTAL OF $<0.05$

0.00 IN A COLUMN INDICATES $A$ TOTAL Of $<0.005$ 


\section{INEL ELECTRONIC TECHNOLOGY CENTER, 1992 DETAIL DATA}

Report 10-Hazardous Waste Generated By IET For January-December 1992 


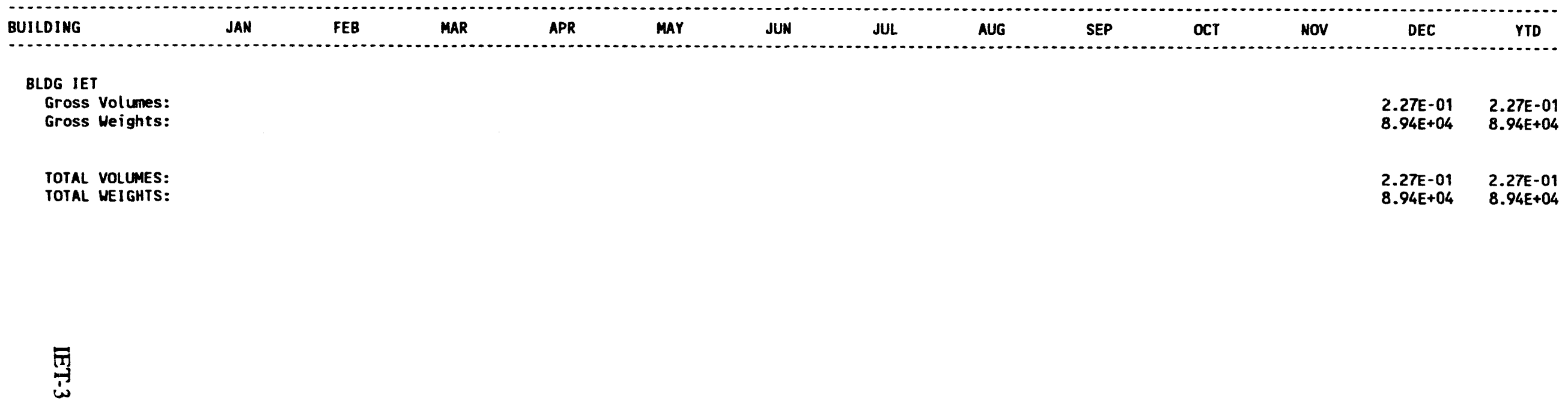




\section{INEL RESEARCH CENTER, 1992 DETAIL DATA}

Report 10-Hazardous Waste Generated By IRC For January-December 1992

IRC-3

Report 11-Mixed Waste Generated By IRC For January-December $1992 \ldots$ IRC-4 
HAZARDOUS WASTE GENERATED BY IRC

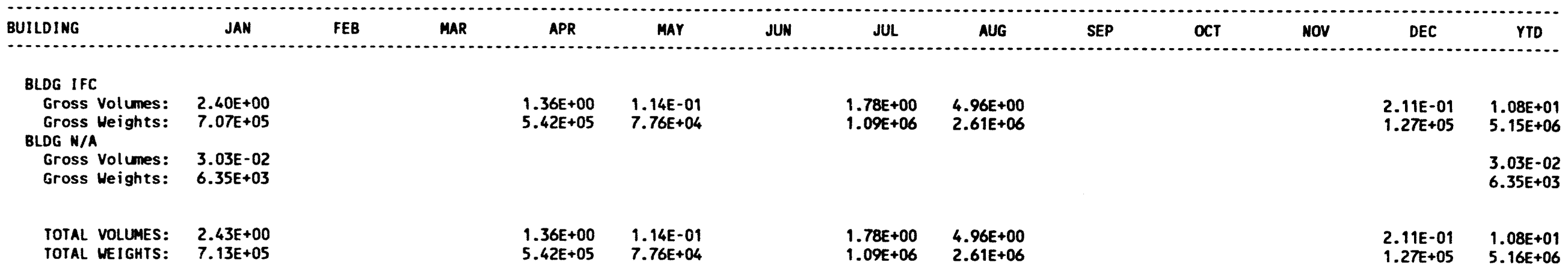

ฺี่

NOTE: VOLUME REPORTED IN CUBIC METERS; WEIGHT REPORTED IN GRAMS DETAILS MAY NOT ADD UP TO TOTALS BECAUSE OF ROUNDING 
MIXED WASTE GENERATED BY IRC

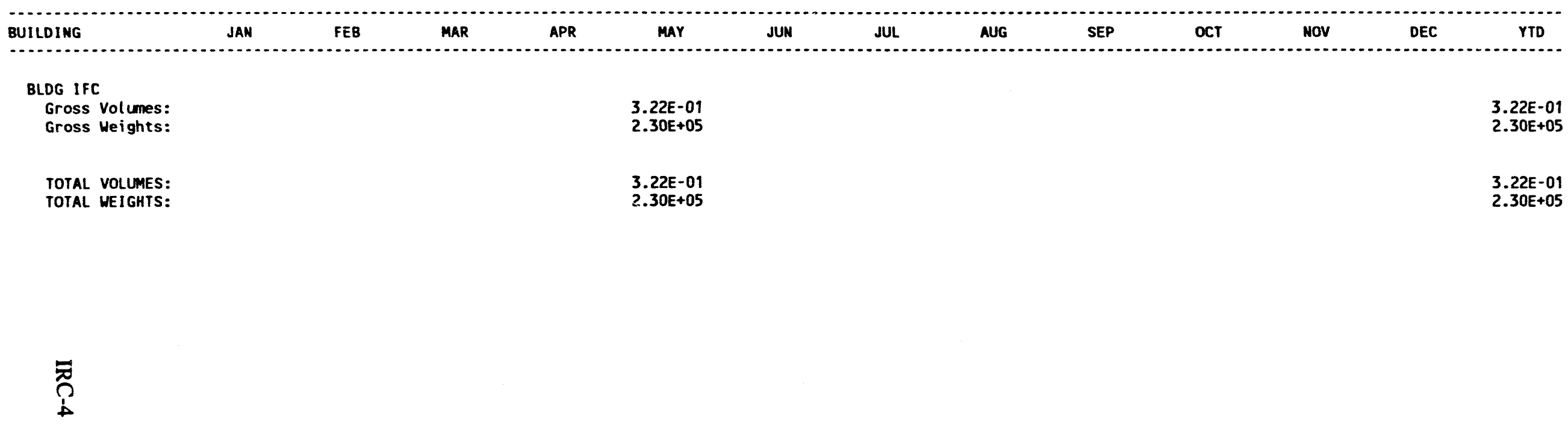




\section{MAGNETOHYDRODYNAMICS COMPONENT DEVELOPMENT,}

1992 DETAIL DATA

Report 176-MHD Landfill Solid Disposed Waste Summary ........... MHD-3

Report 177-MHD Water Usage And Disposal Summary For January

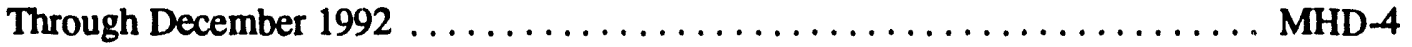

Report 178-MHD Fuel Oil Usage And Stack Effluents Summary . . . . . . . . MHD-6 
INEL NONRADIOLOGICAL MASTE MANAGEMENT INFORMATION SYSTEM

FOR JANUARY THROUGH DECEMBER 1992

MHD LANDFILL SOLID DISPOSED WASTE SUMMARY

(ALL VOLUMES $=$ CUBIC METERS)

\begin{tabular}{|c|c|c|c|c|c|c|c|c|c|c|c|c|c|}
\hline Type of Waste & JAN & FEB & MAR & APR & MAY & JUN & JUL & AUG & SEP & OCT & NOV & DEC & TOTALS \\
\hline TRASH， SWEEPINGS, ETC. & 73.4 & 73.4 & & & & & & & & & & & 146.8 \\
\hline GRAND TOTALS: & 73.4 & 73.4 & & & & & & & & & & & 146.8 \\
\hline
\end{tabular}


FOR JAMUARY THROUGH DECEMBER 1992

MHD WATER USAGE AND DISPOSAL SUMMARY

MHD

(ALL VOLUMES = NEAREST THOUSAND LITERS)

ANNUAL TOT

WELL OR BLDG. NO.I
SEWAGE BLDG. NO.

WATER DATA

N/A HATER PUMPED (L)

$312 \quad 388$

700

WATER PUMPED (L)

WATER DISPOSED

$\begin{array}{lrr}\text { AIR (L) } & 32 & 26 \\ \text { SURFACE (L) } & 280 & 361 \\ \text { SUBSURFACE (L) } & \end{array}$

SUBSURFACE (L)

SEWAGE DATA

BUILD VOL (L)

BIOCHEMICAL OXYGEN DEMAND (BCO)

RAW CONC. (MG/L)

FINAL CONC. (MG/L)

$X$ REMTIVED

DISSOLVED OXYGEN (DO)

RAH CONC. (MG/L)

FINAL CONC. (MG/L)

INCREASED FACTOR

SETTLEABLE SOLIDS (SS)

RAW CONC. (ML/L)

FINAL CONC. (ML/L)

\% REMOVED

PH FINAL

TOTAL SEWAGE(L)

TOTAL WATER DISPOSED(L)

DETAILS MAY NOT ADD UP TO TOTALS BECAUSE OF ROUNDING

0 IN A COLUMN INDICATES $A$ TOTAL OF $<0.5$

0.0 IN A COLUMN INDICATES A TOTAL OF $<0.05$

0.00 IN A COLUMN INDICATES $A$ TOTAL OF $<0.005$ 
PAGE : 2

SCHED. NO. INRPTO1O
IDAHO OPERATIONS OFFICE

UNITED STATES DEPARTMENT OF ENERGY

INEL NONRADIOLOGICAL WASTE MAMAGEMENT INFORMATION SYSTEM

FOR JANUARY THROUGH DECEMBER 1992

MHD WATER USAGE AND DISPOSAL SUMAARY

(ALL VOLUMES = NEAREST THOUSAMD LITERS)

RPT $177-1$

MHD

ANNUAL TOT
VOL/WEIGHT

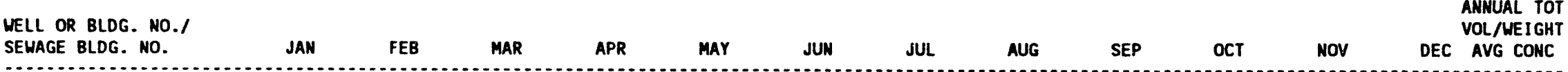

SEHAGE BLDG. NO.

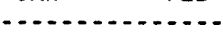

ACCOUNTABILITY (X) $\quad 100.0 \quad 100.0$

100.0 
PAGE: 1

IDAHO OPERATIONS OFFICE

19- JUN-93

SCHED. NO. INRPTOII

UNITED STATES DEPARTMENT OF ENERGY

INEL NONRADIOLOGICAL WASTE MAMAGEMENT IMFORMATION SYSTEM

FOR JANUARY THROUGH DECEMBER 1992

MHD FUEL OIL USAGE AND STACK EFFLUENTS SUMMARY

RPT 178-1

(ALL VALUES = MEAREST THOUSAND)

FUEL OIL TYPE
EFFLUENTS

TYPE 2

VOLUME (LITERS)

SO2 (KGS)

$10 \quad 1$

NOX (KGS)

GRAND TOTALS:

VOLUME (LITERS)

SO2 (KGS)

NOX (KGS)

PARTICULATES (KGS) 
NAVAL REACTORS FACILITY, 1992 DETAIL DATA

Report 184-NRF Airborne Release Point And Disposed Substances

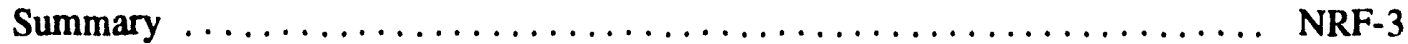

Report 185 -NRF Liquid Disposed Substances Summary . . . . . . . . . . . NRF-4

Report 185-NRF Release Point And Liquid Disposed Substances Summary ... NRF-5

Report 186-NRF Landfill Solid Disposed Waste Summary . . . . . . . . . NRF-7

Report 187-NRF Water Usage And Disposal Summary For January

Through December 1992

NRF-8

Report 188-NRF Fuel Oil Usage And Stack Effluents Summary . . . . . . . . NRF-10

Report 1810-Hazardous Waste Generated By NRF For January-December 1992 NRF-11 
DLOGICAL WASTE MAMAGEMENT IMFORMATION SYSTEM

FOR JAMUARY THROUGH DECEMBER 1992

NRF AIRBORNE RELEASE POINT AMD DISPOSED SUBSTAMCES SUmmaRY

RPT 184-I

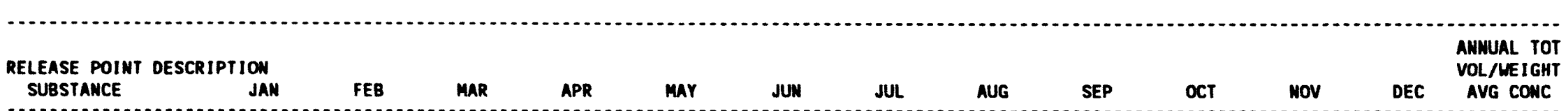

COOLING TONERS

VOLUNE (1000 CN)

29

27

49

35

17

11

409 
PAGE: 1

SCHED. NO. INRPT008A

IDAHO OPERATIONS OFFICE

25-JUN-93

UNITED STATES DEPARTMENT OF ENERGY

INEL NONRADIOLOGICAL WASTE MAMAGEMENT INFORMATION SYSTEM

FOR JANUARY THROUGH DECEMBER 1992

MRF LIQUID DISPOSED SUBSTANCES SUMAARY

RPT 185-1

MRF

\begin{tabular}{|c|c|c|c|c|c|c|c|c|c|c|c|c|c|}
\hline SUBSTANCE & JAN & FEB & MAR & APR & MaY & JUW & JUL & AUG & SEP & OCT & NOV & DEC & $\begin{array}{l}\text { ANMUAL } \\
\text { TOTALS }\end{array}$ \\
\hline $\begin{array}{l}\text { BENZOTRIAZOLE } \\
\text { WEIGHT (KGS) }\end{array}$ & 9 & 19 & 13 & 14 & 13 & 16 & 11 & 20 & 19 & 18 & 46 & 25 & 224 \\
\hline $\begin{array}{l}\text { CALCIUM ION } \\
\text { WEIGHT (KGS) }\end{array}$ & 443 & 30 & 26 & $4 \bar{z}$ & 17 & 23 & 23 & 28 & 32 & 11 & 9 & 9 & 691 \\
\hline $\begin{array}{l}\text { CHLORIDE ION } \\
\text { WEIGHT (KGS) }\end{array}$ & 9,385 & 23,613 & 11,567 & 7,387 & 8,884 & 21,085 & 10,782 & 13,454 & 15,278 & 11,213 & 10,167 & 11,752 & 154,567 \\
\hline $\begin{array}{l}\text { HYPOCHLORITE ION } \\
\text { MEIGHT (KGS) }\end{array}$ & 1,137 & 78 & 66 & 108 & 43 & 59 & 58 & 72 & 82 & 27 & 24 & 23 & 1,776 \\
\hline $\begin{array}{l}\text { PHOSPHATE IOM } \\
\text { WEIGHT (KGS) }\end{array}$ & 44 & 113 & 52 & 82 & 75 & 73 & 47 & 105 & 127 & 32 & 47 & 62 & 858 \\
\hline $\begin{array}{l}\text { SOOIUM HYDROXIDE } \\
\text { WEIGHT (KGS) }\end{array}$ & 27 & 57 & 40 & 43 & 38 & 49 & 33 & 60 & 56 & 54 & 139 & 74 & 671 \\
\hline $\begin{array}{l}\text { SULFATE ION } \\
\text { WEIGHT (KGS) }\end{array}$ & 4,678 & 15,980 & 16,439 & 11,032 & 11,922 & 17,627 & 9,233 & 27,464 & 47,382 & 13,365 & 11,571 & 20,873 & 207,566 \\
\hline
\end{tabular}




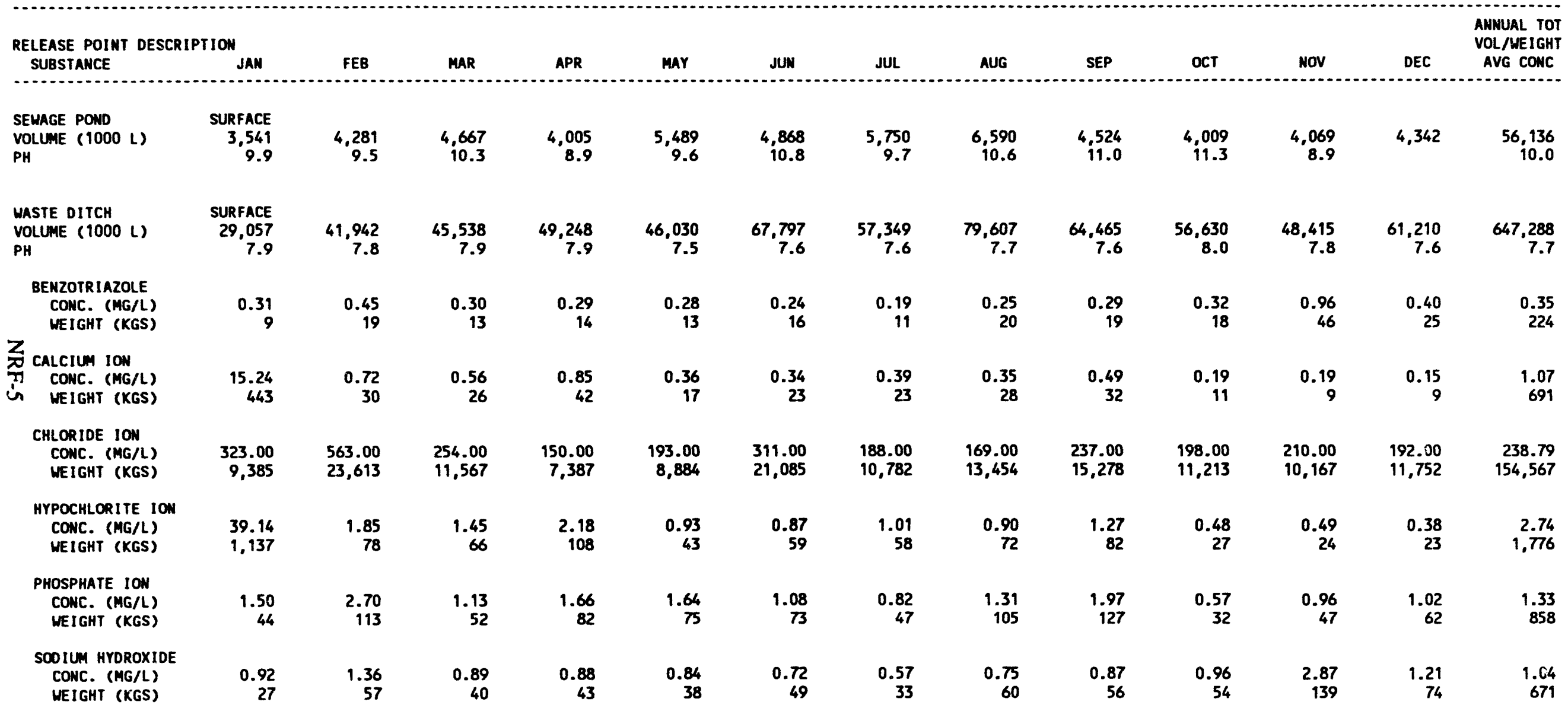


PAGE: 2

SCHED. NO. INRPTOOB
IDAHO OPERATIONS OFFICE

UNITED STATES DEPARTMENT OF ENERGY

INEL NONRADIOLOGICAL WASTE MANAGEMENT IMFORMATIOW SYSTEM

FOR JAMUARY THROUGH DECEMBER 190?

NRF RELEASE POINT AND LIQUID DISPOSED SUBSTANCES SUMMARY
25-JUN-93

RPT 185-I

\begin{tabular}{|c|c|c|c|c|c|c|c|c|c|c|c|c|c|}
\hline $\begin{array}{l}\text { RELEASE POINT DESC } \\
\text { SUBSTANCE }\end{array}$ & TION JAN $_{\text {JAN }}$ & FEB & MAR & APR & MAY & JUN & JUL & AUG & SEP & OCT & NOV & DEC & $\begin{array}{l}\text { ANMUAL TOT } \\
\text { VOL/NEIGHT } \\
\text { AVG CONC }\end{array}$ \\
\hline $\begin{array}{l}\text { WASTE DITCH } \\
\text { SOOIUA IOW }\end{array}$ & SURFACE & & & & & & & & & & & & \\
\hline $\begin{array}{l}\text { CONC. (MG/L) } \\
\text { MEIGHT (KGS) }\end{array}$ & $\begin{array}{r}311.00 \\
9,037\end{array}$ & $\begin{array}{l}309.00 \\
12,960\end{array}$ & $\begin{array}{l}250.00 \\
11,385\end{array}$ & $\begin{array}{r}108.00 \\
5,319\end{array}$ & $\begin{array}{l}99.60 \\
4.585\end{array}$ & $\begin{array}{r}115.00 \\
7,797\end{array}$ & $\begin{array}{r}159.00 \\
9.118\end{array}$ & $\begin{array}{l}77.60 \\
6.178\end{array}$ & $\begin{array}{r}111.00 \\
7,156\end{array}$ & $\begin{array}{l}89.50 \\
5,068\end{array}$ & $\begin{array}{r}147.00 \\
7.117\end{array}$ & $\begin{array}{r}103.00 \\
6,305\end{array}$ & $\begin{array}{l}142.17 \\
92,023\end{array}$ \\
\hline $\begin{array}{l}\text { SULFATE ION } \\
\text { CONC. (MG/L) } \\
\text { WEIGHT (KGS) }\end{array}$ & $\begin{array}{r}161.00 \\
4.678\end{array}$ & $\begin{array}{l}381.00 \\
15,980\end{array}$ & $\begin{array}{l}361.00 \\
16,439\end{array}$ & $\begin{array}{l}224.00 \\
11.032\end{array}$ & $\begin{array}{l}259.00 \\
11,922\end{array}$ & $\begin{array}{l}260.00 \\
17,627\end{array}$ & $\begin{array}{r}161.00 \\
9,233\end{array}$ & $\begin{array}{l}345.00 \\
27.464\end{array}$ & $\begin{array}{l}735.00 \\
47,382\end{array}$ & $\begin{array}{l}236.00 \\
13,365\end{array}$ & $\begin{array}{l}239.00 \\
11,571\end{array}$ & $\begin{array}{l}341.00 \\
20,873\end{array}$ & $\begin{array}{r}320.67 \\
207,566\end{array}$ \\
\hline
\end{tabular}


NRF LANDFILL SOLID DISPOSED HASTE SUMMARY

(ALL VOLUMES = CUBIC METERS)

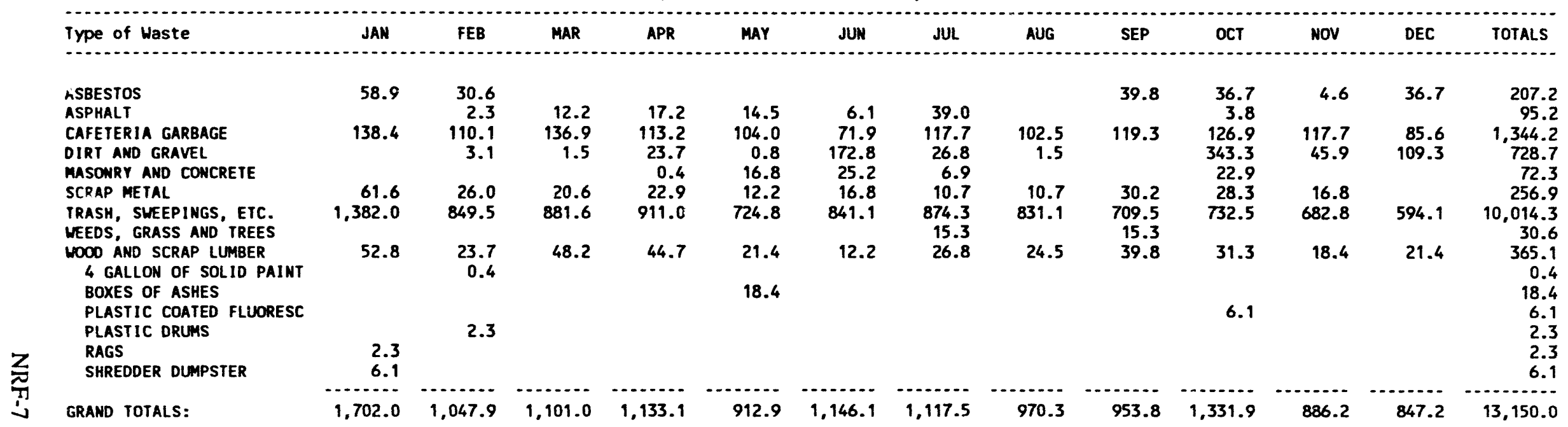


PAGE: 1

SCHED. NO. INRPTO10

IDAHO OPERATIONS OFFICE

UNITED STATES DEPARTMENT OF EMERGY

19-JUN-93

INEL NONRADIOLOGICAL WASTE MANAGEMENT IMFORMATION SYSTEM

FOR JANUARY THROUGH DECEMBER 1992

NRF WATER USAGE AND DISPOSAL SUMMARY

RPT 187-1

(ALL VOLUMES = NEAREST THOUSAND LITERS )

NRF

MELL OR BLDG. NO.I
SEWAGE BLDG. NO.

\section{WATER DATA}

001 WATER PUMPED (L)

OO2 WATER PUMPED (L)

003 WATER PUMPED (L)

004 WATER PUMPED (L)

\begin{tabular}{rrrr}
276 & 8 & 197 & 3,392 \\
47,885 & 50,308 & 45,879 & 39,860 \\
1,136 & 38 & 53 & 413 \\
20,188 & 16,436 & 33,130 & 36,514 \\
\hline
\end{tabular}

WATER PUMPED (L)

$69,485 \quad 66,790 \quad 79,259$

80,179

$5,061 \quad 19,071$

10,372

$661 \quad 51,974$

38

16.470

36,279

30,946

8
85,399

8212

212
307

$\begin{array}{rrrrr}19 & 11 & 216 & 38,842\end{array}$

988

MATER DISPOSED

AIR (L)

SURFACE (L)
SUBSURFACE (L)

36,885

36,885
29,057

26,895

48,785

$80,444 \quad 134,041 \quad 185,822$

250

$\begin{array}{rrr}41,299 & 71,658 & 667,347 \\ 8 & 45 & 2,809\end{array}$

$\frac{2}{0}$

SEWAGE DATA

722 BUILD VOL (L)

$$
3,541 \quad 4,281
$$

4,667

4,005

5,489

4,868

5,750

6,590

4,524

4,009

56,136

BIOCHEMICAL OXYGEN DEMAND (BOD)

RAW CONC. (MG/L)

77.0

14.0

48.0

28.0

24.0

33.0

21.0

23.0

33.5

DISSOLVED OXYGEN (DO)

RAH CONC. (MG/L)

FINAL CONC. (MG/L)

\section{SETTLEABLE SOLIDS (SS)}

RAW CONC. (ML/L)

FINAL CONC. (ML/L)

X REMOVED

PH FINAL 
PAGE : 2

SCHED. NO. INRPTO1O

IDAHO OPERATIONS OFFICE

UNITED STATES DEPARTMENT OF ENERGY

19- JUN-93

INÉL NONRADIOLOGICAL WASTE MAMAGEMENT INFORMATION SYSTEM

FOR JANUARY THROUGH DECEMBER 1992

MRF WATER USAGE AND DISPOSAL SUMWARY

RPT 187-I

(ALL VOLUMES = NEAREST THOUSAMD LITERS)

NRF

\begin{tabular}{|c|c|c|c|c|c|c|c|c|c|c|c|c|c|}
\hline $\begin{array}{l}\text { WELL OR BLDG. NO.I } \\
\text { SEWAGE BLDG. NO. }\end{array}$ & JAN & FEB & MAR & APR & MAY & JUN & JUL & AUG & SEP & OCT & NOV & DEC & $\begin{array}{l}\text { ANNUAL TOT } \\
\text { VOL/WEIGHT } \\
\text { AVG CONC }\end{array}$ \\
\hline TOTAL WATER DISPOSED(L) & 69,483 & 66,756 & 79,259 & 80,148 & 100,237 & 107,789 & 80,417 & 134,045 & 185,806 & 61,635 & 70,378 & 76,821 & $1,112,774$ \\
\hline ACCOUNTABILITY $(x)$ & 100.0 & 99.9 & 100.0 & 100.0 & 100.0 & 100.0 & 100.0 & 100.0 & 100.0 & 103.3 & 100.0 & 100.0 & 100.2 \\
\hline
\end{tabular}

DETAILS MAY NOT ADD UP TO TOTALS BECAUSE OF ROUNDING

O IN A COLLMN INDICATES A TOTAL OF $<0.5$

0.0 IN A COLUMN INDICATES A TOTAL OF $<0.05$

0.00 IN A COLUMN INDICATES A TOTAL OF $<0.005$ 
PAGE: 1

SCHED. NO. INRPTO11

IDAHO OPERATIONS OFFICE

19-JUN-93

UNITED STATES DEPARTMENT OF ENERGY

INEL NONRADIOLOGICAL WASTE MANAGEMENT INFORMATION SYSTEM

FOR JANUARY THROUGH DECEMBER 1992

NRF FUEL OIL USAGE AND STACK EFFLUENTS SUMMARY

RPT 188-1

(ALL VALUES = MEAREST THOUSAMD)

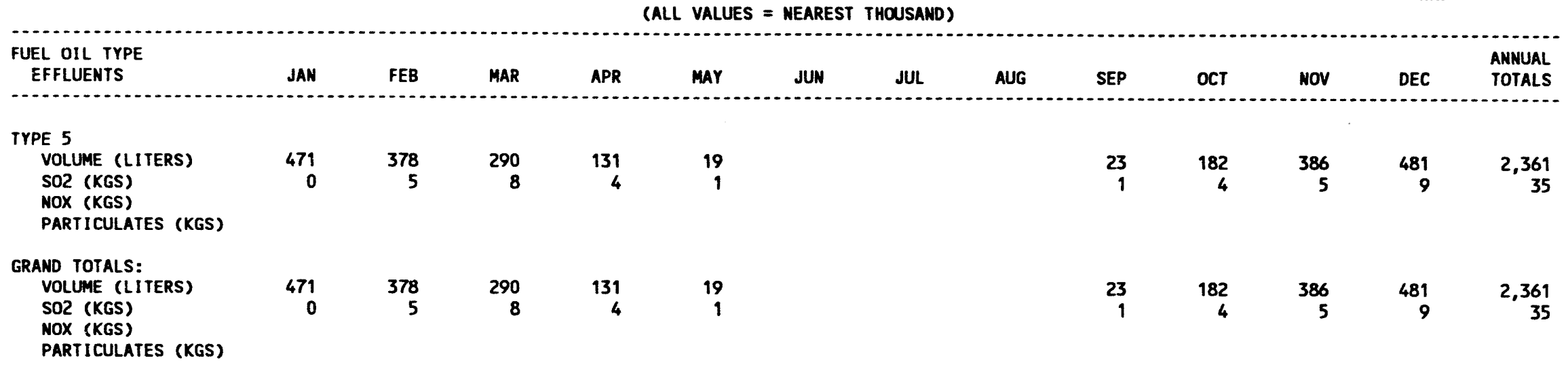

竞

0 IN A COLUAN INDICATES A TOTAL OF $<0.5$ 


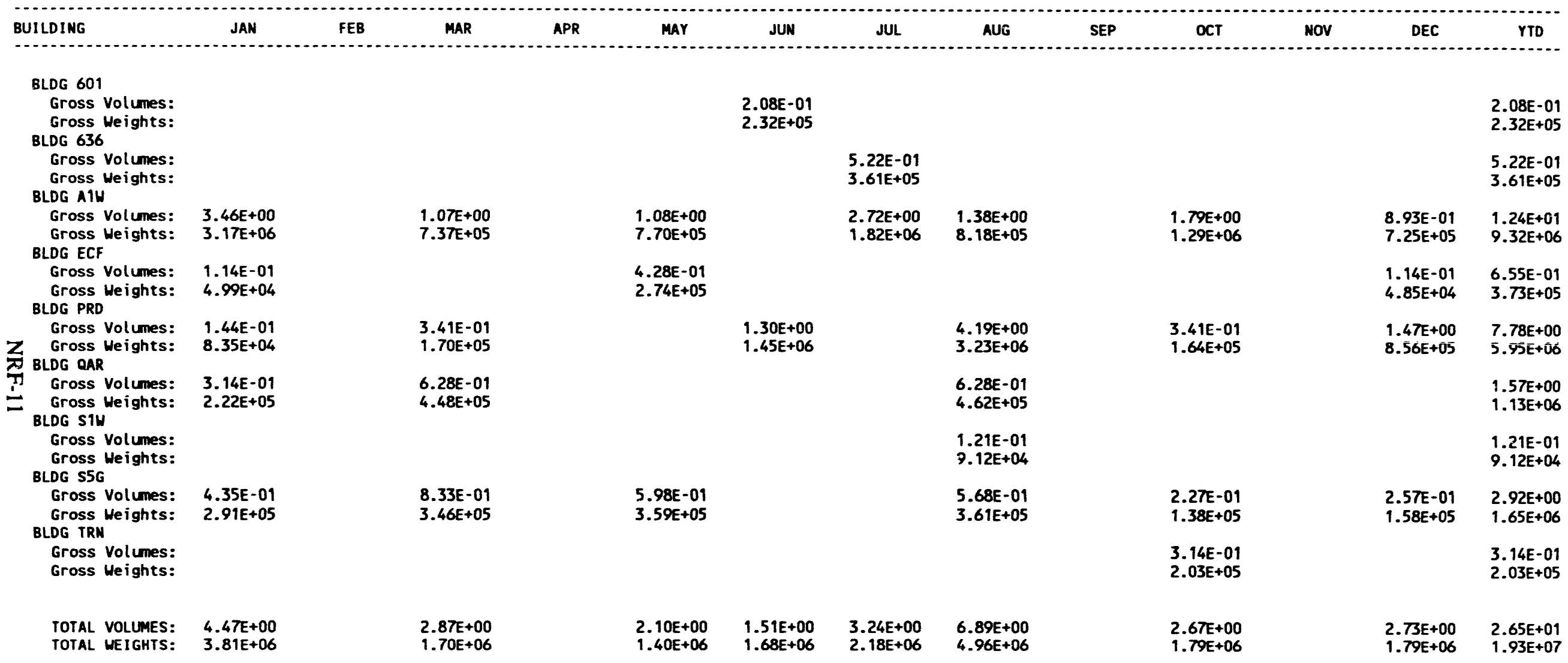




\section{POWER BURST FACILITY, 1992 DETAIL DATA}

Report 195-PBF Liquid Disposed Substances Summary ............. PBF-3

Report 195-PBF Release Point And Liquid Disposed Substances Summary . . . PBF-4

Report 196-PBF Landfill Solid Disposed Waste Summary ........... PBF-6

Report 197-PBF Water Usage And Disposal Summary For January

Through December $1992 \ldots \ldots \ldots \ldots \ldots \ldots \ldots \ldots \ldots \ldots \ldots \ldots$. PBF-7

Report 198_PBF Fuel Oil Usage And Stack Effluents Summary . . . . . . . . PBF-9

Report 1910—Hazardous Waste Generated By PBF For January-December

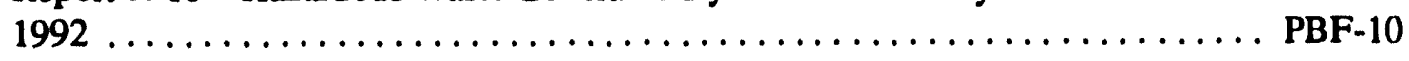


PBF LIQUID DISPOSED SUBSTANCES SUMmARY

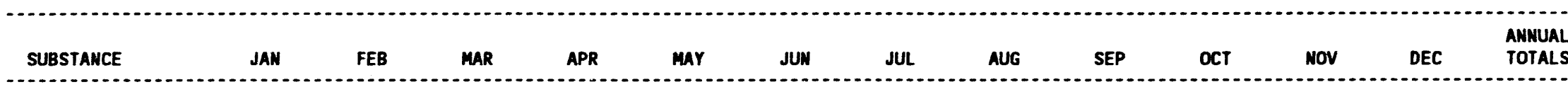

ETHYLENEDIAMINE TETRAACETIC ACID WEIGHT (KGS)

JANITORIAL SUPPLIES MEIGHT (KGS) 0

POTASSIUN HYDROXIDE WEIGHT (KGS)

0

\section{SOOIUM ION}

WEIGHT (KGS)

SODIUM LIGNOSULFOMATE

WEIGHT (KGS)

SULFITE IO

WEIGHT (KGS)

$$
12
$$

0

o
0

$\begin{array}{lllll}0 & 0 & 0 & 1 \\ 11 & 9 & 6 & 12 & 57 \\ 0 & 0 & 0 & 1 \\ 0 & 0 & 0 & 1 \\ 0 & 0 & 0 & 1 \\ 0 & & & 1\end{array}$




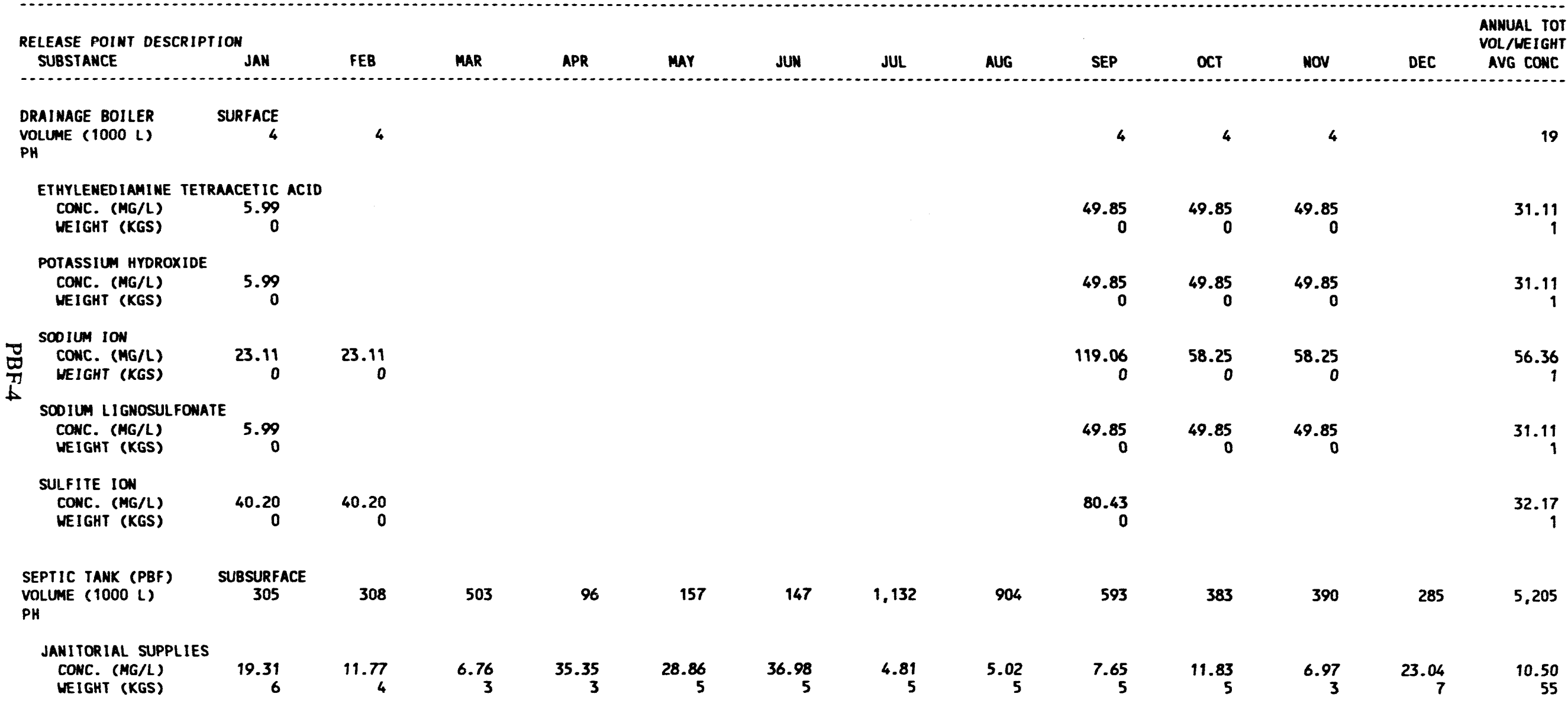

\footnotetext{
SEPTIC TANK (WERF) SUBSURFACE
} 
OLOGICAL MASTE MAMAGEMENT INFORMATION SYSTEM

FOR JAMUARY IHROUGH DECEMBER 1992

PBF RELEASE POINT AND LIQUID DISPOSED SUBSTANCES SUMMARY

RPT 195-I

PBF

\begin{tabular}{|c|c|c|c|c|c|c|c|c|c|c|c|c|c|}
\hline $\begin{array}{l}\text { RELEASE POINT DESCRIP } \\
\text { SUBSTANCE }\end{array}$ & TION JAN & FEB & MAR & APR & MAY & JUN & JUL & AUG & SEP & OCT & NOV & DEC & $\begin{array}{l}\text { ANMUAL TOT } \\
\text { VOL/WEIGHT } \\
\text { AVG CONC }\end{array}$ \\
\hline $\begin{array}{l}\text { SEPTIC TANK (MERF) } \\
\text { VOLUME }(1000 \mathrm{~L}) \\
\text { PH }\end{array}$ & $\begin{array}{l}\text { SUBSURFACE } \\
153\end{array}$ & 154 & 252 & 48 & 79 & 74 & 377 & 452 & 296 & 192 & 195 & 571 & 2,841 \\
\hline $\begin{array}{l}\text { JANI TORIAL SUPPLIES } \\
\text { CONC. (MG/L) } \\
\text { WEIGHT (KGS) }\end{array}$ & $\begin{array}{r}41.59 \\
6\end{array}$ & $\begin{array}{r}23.54 \\
4\end{array}$ & $\begin{array}{r}18.04 \\
5\end{array}$ & $\begin{array}{r}94.28 \\
5\end{array}$ & $\begin{array}{r}69.26 \\
5\end{array}$ & $\begin{array}{r}43.15 \\
3\end{array}$ & $\begin{array}{r}8.43 \\
3\end{array}$ & $\begin{array}{r}14.05 \\
6\end{array}$ & $\begin{array}{r}21.43 \\
6\end{array}$ & $\begin{array}{r}23.66 \\
5\end{array}$ & $\begin{array}{r}16.28 \\
3\end{array}$ & $\begin{array}{r}9.54 \\
5\end{array}$ & $\begin{array}{r}19.96 \\
57\end{array}$ \\
\hline
\end{tabular}

$\underset{\varpi}{\square}$

O IN A COLUNA IMDICATES A TOTAL OF $<0.5$

0.00 IN A COLUHA INDICATES A TOTAL OF $<0.005$ 
PAGE: 1

SCHED. NO. INRPTOOS
IDAHO OPERATIONS OFFICE

UNITED STATES DEPARTMENT OF ENERGY

INEL NONRADIOLOGICAL HASTE MANAGEMENT INFORMATION SYSTEM

FOR JANUARY THROUGH DECEMBER 1992

PBF LANDFILL SOLID DISPOSED MASTE SUMMARY

RPT 196-I

PBF

(ALL VOLUMES = CUBIC METERS)

\begin{tabular}{|c|c|c|c|c|c|c|c|c|c|c|c|c|c|}
\hline Type of Waste & JAN & FEB & MAR & APR & MAY & JUN & JUL & AUG & SEP & OCT & NOV & DEC & TOTALS \\
\hline $\begin{array}{l}\text { ASBESTOS } \\
\text { ASPHALT }\end{array}$ & 2.7 & & & & & & 15 & & 0.8 & & & & $\begin{array}{l}3.4 \\
1.5\end{array}$ \\
\hline $\begin{array}{l}\text { CAFETERIA GARBAGE } \\
\text { DIRT AND GRAVEL }\end{array}$ & & & $\begin{array}{r}12.2 \\
1.1\end{array}$ & & & & & & & & & & $\begin{array}{r}12.2 \\
1.1\end{array}$ \\
\hline $\begin{array}{l}\text { MASONRY AND CONCRETE } \\
\text { SCRAP METAL }\end{array}$ & C.8 & & 4.6 & $\begin{array}{l}1.5 \\
1.1\end{array}$ & & & 0.8 & 12.6 & $\begin{array}{l}1.9 \\
1.1\end{array}$ & 1.9 & 0.4 & 1.5 & $\begin{array}{r}3.4 \\
24.8\end{array}$ \\
\hline $\begin{array}{l}\text { TRASH, SWEEPINGS, ETC. } \\
\text { WEEDS, GRAS AND TREES }\end{array}$ & 63.5 & 45.9 & $\begin{array}{r}52.0 \\
0.4\end{array}$ & 66.5 & 61.6 & $\begin{array}{r}41.7 \\
2.3\end{array}$ & $\begin{array}{r}45.9 \\
0.8\end{array}$ & 39.0 & $\begin{array}{r}52.0 \\
9.2\end{array}$ & 61.9 & 49.7 & 58.1 & $\begin{array}{r}637.7 \\
12.6\end{array}$ \\
\hline $\begin{array}{l}\text { WOOD AND SCRAP LUMBER } \\
\text { PLASTIC }\end{array}$ & $\begin{array}{l}1.5 \\
0.4\end{array}$ & 0.4 & & 0.8 & 2.3 & 7.6 & 1.5 & 2.7 & 9.9 & 9.6 & & & $\begin{array}{r}36.3 \\
0.4\end{array}$ \\
\hline GRAND TOTALS: & 68.8 & 46.3 & 70.3 & 70.0 & 63.8 & 51.6 & 50.5 & 54.3 & 74.9 & 73.4 & 50.1 & 59.6 & 733.6 \\
\hline
\end{tabular}

$\frac{7}{0}$ 
INEL NONRADIOLOGITALATES DEPARTMENT OF EMERGY

RPT 197-1

FOR JANUARY THROUGH DECEMBER 1992

PBF MATER USAGE AND DISPOSAL SUMMARY

PBF

(ALL VOLUMES = MEAREST THOUSAND LITERS)

ANHUAL TOT

VOL/NEIGHT

WELL OR BLDG. NO.I

JAN FEB MAR APR

Mar

JUN

JUL

AUG

SEP

OCT

NOV DEC AVG CONC

MATER DATA

001 WATER PUMPED (L)

O02 WATER PUMPED (L)

WATER PUMPED (L)

\begin{tabular}{rrrr}
648 & 340 & 613 \\
200 & 517 & 784 \\
\hline 848 & & 857 & 1,397
\end{tabular}

613
784
397

$\begin{array}{rr}110 & 1,524 \\ 157 & 2,842 \\ 267 & 4,366\end{array}$

329
80
409

\begin{tabular}{rr}
1,495 & 610 \\
598 & 1,901 \\
\hline 2,093 & 2,511
\end{tabular}

$\begin{array}{r}929 \\ 717 \\ \hline 1,646\end{array}$

$\begin{array}{r}712 \\ 354 \\ \hline 1,065\end{array}$

\begin{tabular}{rrr}
397 & 418 & 8,124 \\
686 & 1,169 & 10,005 \\
\hline 1,084 & 1,586 & 18,129
\end{tabular}

AIR (L)

AIR (L)

SURFACE (L)
SUBSURFACE (L)

254

257

419

$80 \quad 1,308$

123

628

494

320

325

476

5,437

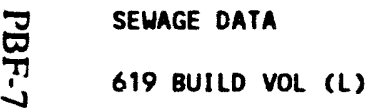

$153 \quad 154 \quad 252$

48

74

377

452

296

192

195

285

3,263

BIOCHEMICAL OXYGEN DEMAND (BOO)

RAH CONC. (MG/L)

FIMAL CONC. (MG/L)

$X$ REMOVED

DISSOLVED OXYGEN (DO)

RAW CONC. (MG/L)

FINAL CONC. (MG/L)

INCREASED FACTOR

SETTLEABLE SOLIDS (SS)

RAW CONC. (ML/L)

FINAL CONC. (ML/L)

X REMOVED

PH FINAL 
PAGE: 2

SCHED. NO. INRPTO10

IDAHO OPERATIONS OFFICE

19-JUN-93

IMEL MOMRADIOLITED STATES DEPARTMEMT OF EMERGY

FOR JAMUARY THROUGH DECEMBER 1992

PBF MATER USAGE AND DISPOSAL SUMUARY

RPT 197-1

PBF

(ALL VOLUMES = MEAREST THOUSAMD LITERS)

ANMUAL TOT

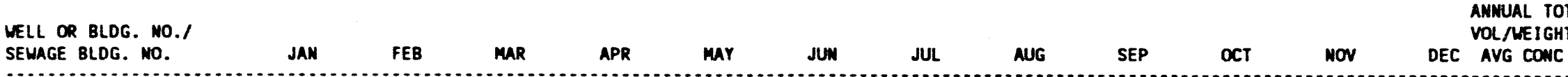

-.................

305

308

503

96

1,572

147

753

904

593

383

390

571

6,527

BIOCHEMICAL OXYGEN DEmano (BCO)

RAW CONC. (MG/L)

FIMAL CONC. (MG/L)

X REMOVED

DISSOLVED OXYGEN (DO)

RAW CONC. (MG/L)

FIHAL CONC. (MG/L)

IMCREASED FACTOR

\section{SETTLEABLE SOLIDS (SS)}

RAW CONC. (ML/L)

FIMAL CONC. (ML/L)

FINAL CONC.

PH FINAL

TOTAL WATER OISPOSED(L)

463

$755 \quad 144$

ACCOUNTABILITY (x)

713

463

1,174

$\begin{array}{ll}144 & 2,358 \\ 225 & 3,666\end{array}$

$\begin{array}{ll}221 & 1,130 \\ 343 & 1,758\end{array}$

1,356
2,109
84.0

889
1,383
84.0

575
895
84.0

$\begin{array}{rrr}585 & 856 & 9.790 \\ 910 & 1.332 & 15.227 \\ 84.0 & 84.0 & 84.0\end{array}$

DETAILS MAY NOT ADO UP TO TOTALS BECAUSE DF ROMNIMG 
INEL NONRADIOLOGICAL WASTE MANAGEMENT INFORMATION SYSTEM

FOR JANUARY THROUGH DECEMBER 1992

PBF FUEL OIL USAGE AND STACK EFFLUENTS SUMmARY

(ALL VALUES $=$ MEAREST THOUSAND)

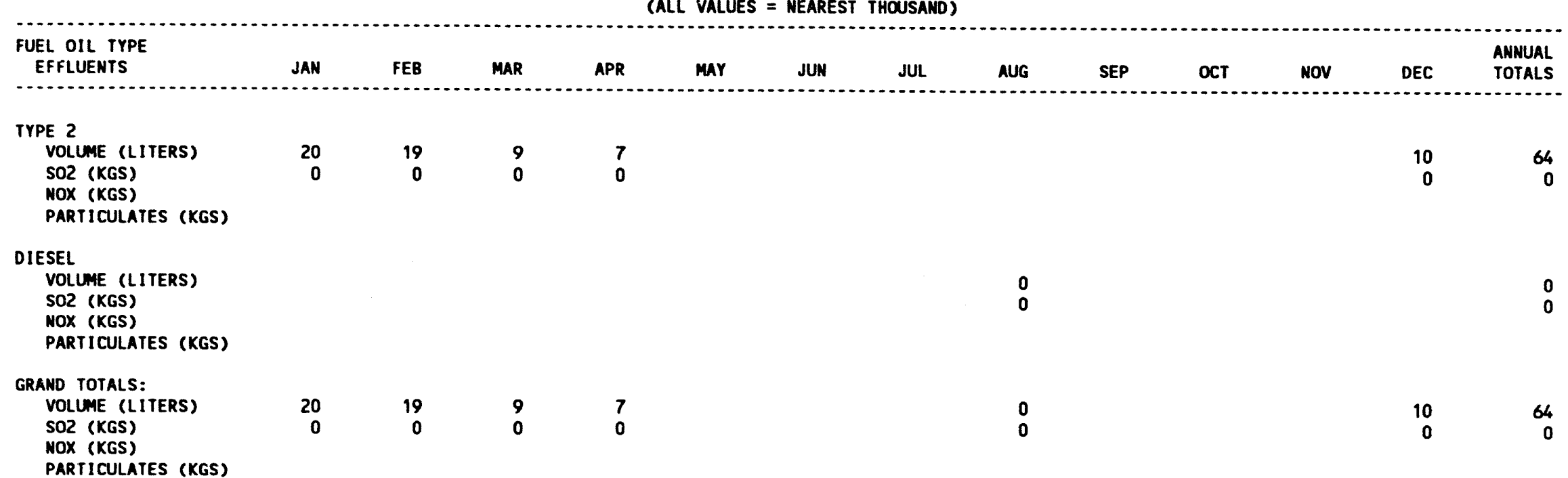


Page: 1

Sched. No. INRPT013

IDAHO OPERATIONS OFFICE

19-JUN-93

UNITED STATES DEPARTMENT OF ENERGY

RPT 1910-I

HAZARDOUS WASTE GENERATED BY PBF

PBF

FOR January - December 1992

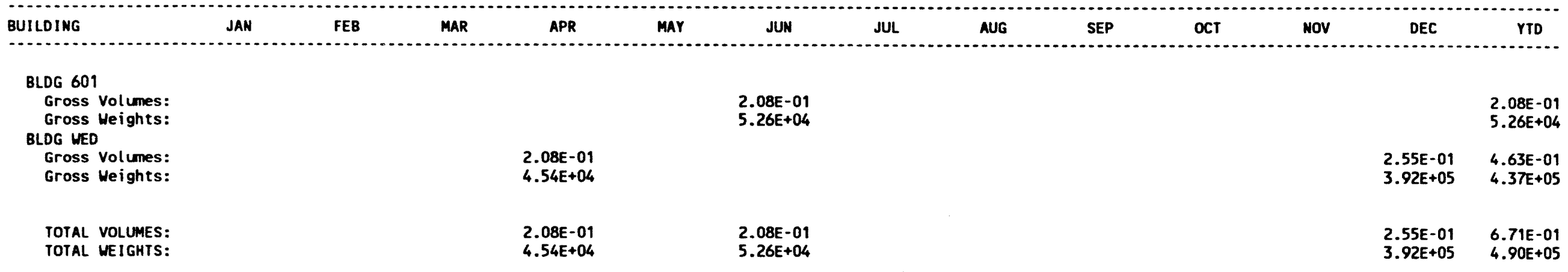

$\frac{0}{0}$

NOTE: VOLUME REPORTED IN CUBIC METERS; WEIGHT REPORTED IN GRAMS DETAILS MAY MOT ADD UP TO TOTALS BECAUSE OF ROUNDING 
MIXED WASTE GENERATED BY PBF

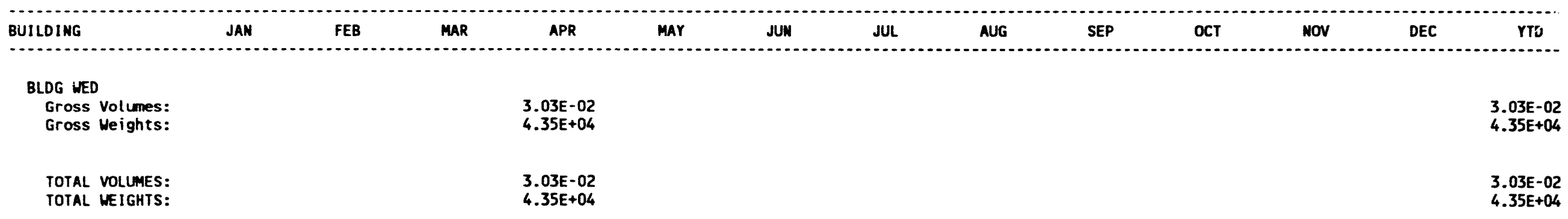

量 
PROTECTION TECHNOLOGY IDAHO, 1992 DETAIL DATA

Report 10-Hazardous Waste Generated By PTI For

January-December 1992

PTI-3

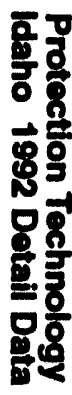


HAZARDOUS WASTE GENERATED BY PTI

FOR January - December 1992

PT

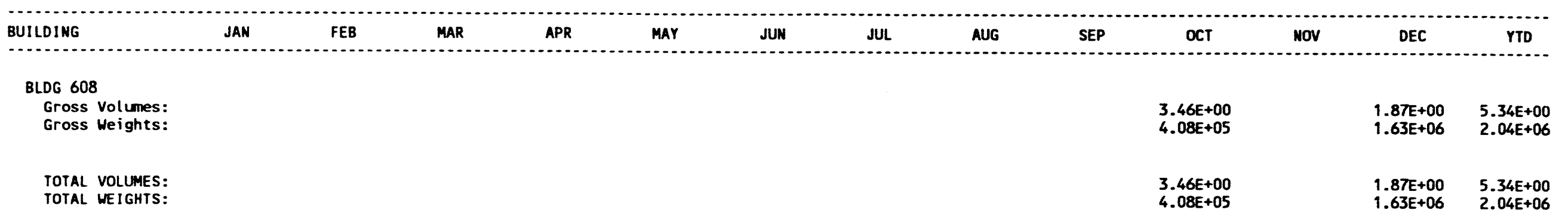


POTABLE WATER TREATMENT UNIT, 1992 DETAIL DATA

Report 305—PWT Release Point And Liquid Disposed

Substances Summary

PWT-3

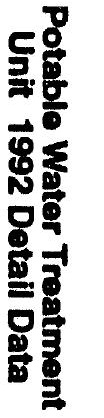

PWT-1 
PUT RELEASE POINT AMD LIOUID DISPOSED SUBSTAMCES SUMAMRY

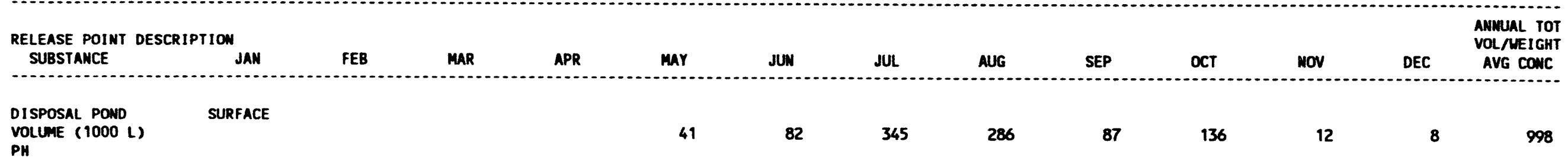


WAC LANDFILL SOLID DISPOSED MASTE SUMWARY

RPT 256-I

RPT

(ALL VOLUMES $=$ CUBIC METERS)

\begin{tabular}{|c|c|c|c|c|c|c|c|c|c|c|c|c|c|}
\hline Type of Waste & JAN & FEB & MAR & APR & maY & JUN & JUL & AUG & SEP & OCT & NOV & DEC & OTALS \\
\hline $\begin{array}{l}\text { ASBESTOS } \\
\text { MASONRY AND CONCRETE } \\
\text { SCRAP METAL } \\
\text { TRASH, SWEEPINGS, ETC. } \\
\text { WOOD AND SCRAP LUMBER } \\
\text { COLOR COOED MATERIAL } \\
\text { FIBER GLASS BOXES \& PLY } \\
\text { FIBER GLASS; SHOVEL }\end{array}$ & 53.5 & $\begin{array}{r}3.1 \\
111.6 \\
0.4 \\
1.9 \\
0.4\end{array}$ & $\begin{array}{r}0.4 \\
59.3\end{array}$ & $\begin{array}{r}5.7 \\
45.9 \\
2.3\end{array}$ & $\begin{array}{r}0.4 \\
38.2\end{array}$ & $\begin{array}{r}2.1 \\
33.3 \\
1.0\end{array}$ & $\begin{array}{r}57.3 \\
3.4\end{array}$ & $\begin{array}{r}9.2 \\
58.1 \\
8.0\end{array}$ & $\begin{array}{r}0.8 \\
3.4 \\
24.5 \\
9.2\end{array}$ & $\begin{array}{r}0.4 \\
1.9 \\
94.8 \\
1.5\end{array}$ & $\begin{array}{r}35.6 \\
1.5\end{array}$ & $\begin{array}{r}0.8 \\
82.6 \\
0.4\end{array}$ & $\begin{array}{r}0.8 \\
0.8 \\
26.6 \\
694.6 \\
27.7 \\
1.9 \\
0.8 \\
0.4\end{array}$ \\
\hline AND TOTALS: & 54.3 & 117.4 & 59.6 & 53.9 & 38.6 & 36.3 & 60.8 & 75.3 & 37.8 & 98.6 & 37.1 & 83.7 & 753.5 \\
\hline
\end{tabular}


PAGE: 1

PAGE: NO. INRPTO1O

IDAHO OPERATIONS OFFICE

UNITED STATES DEPARTMENT OF ENERGY

19- JUN-93

INEL NONRADIOLOGICAL WASTE MAMAGEMEUT IMFORMATION SYSTEM

FOR JAMUARY THROUGH DECEMBER 1992

WMC WATER USAGE AND DISPOSAL SUMMARY

RPT 257-I

MeT

(ALL VOLUAES = MEAREST THOUSAND LITERS)

AMNUAL TOT

VOL/MEIGHT

WELL OR BLDG. NO./

JAN FEB MAR APR

MaY

JUL

AUG

SEP

OCT

NOV DEC AVG CONC

MATER DATA

IMC WATER PUMPED (L)

\begin{tabular}{|c|c|c|c|c|c|c|c|c|c|c|c|c|}
\hline 222 & 74 & 400 & 74,512 & 75,329 & 545 & 1,415 & 1,384 & 2,132 & 729 & 53 & 365 & 157,161 \\
\hline 222 & 74 & 400 & 74,512 & 75,329 & 545 & 1,415 & 1,384 & 2,132 & 729 & 53 & 365 & 157,161 \\
\hline
\end{tabular}

WATER DISPOSED

AIR (L)
SURFACE (L)
SUBSURFACE (L)

SEMAGE DATA

WMC BUILD VOL $(L$

20

67

360

224

678

65

70

65

107

89

BIOCHEMICAL OXYGEN DEMAND (BOD)

RAW CONC. (MG/L)

FINAL CONC. (MG/L)

X REMCVED

DISSOLVED OXYGEN (DO)

RAH CONC. (MG/L)

FINAL CONC. (MG/L)

INCREASED FACTOR

SETTLEABLE SOLIDS (SS)

RAH CONC. (ML/L)

RAW CONC. (ML/L)

X REMOVED

\section{PH FINAL}

TOTAL SEWAGE(L)

$224 \quad 678$

65

70

65

107

89

7

7

$62 \quad 1,814$

DETAILS MAY MOT ADD UP TO TOTALS BECAUSE OF ROUNDIMG

0 IN A COLUNA INDICATES A TOTAL OF $<0.5$

0.0 IN A COLUN IMDICATES $A$ TOTAL OF $<0.05$

0.0 IN A COLUN ImDICATES A TOTAL OF $<0.05$
0.00 IN A COLUIM IMDICATES A TOTAL OF $<0.005$ 
PAGE: 2

SCHED. NO. INRPTO1O

IDAHO OPERATIONS OFFICE

UNITED STATES DEPARTMENT OF EMERGY

RPT 257-1

FOR JAMUARY THROUGH DECEMBER 1992

WMC HATER USAGE AND DISPOSAL SUMALARY

(ALL VOLUMES = NEAREST THOUSAND LITERS)

AMNUAL TOT

VOL/NEIGHT

WELL OR BLDG. NO.I

SEWAGE BLDG. NO.

JAN

FEB

MAR

APR

MAY

JUN

JUL AUG

SEP

OCT

NOV

DEC AVG CONC

ACCOUNTABILITY (צ)

$9.0 \quad 90.0$

90.0

0.3

0.9

12.0

4.9

4.7

5.0

12.2

13.0

17.1

1.2 
Page: 1

Sched. No. INRPT013A
IDAHO OPERATIONS OFFICE

UNITED STATES DEPARTMENT OF EMERGY

19-JUN-93

INEL NONRADIOLOGICAL WASTE MAMAGEMENT INFORMATION SYSTEM
MIXED MASTE GEMERATED BY MMC

FOR January - December 1992
RPT 2519-I

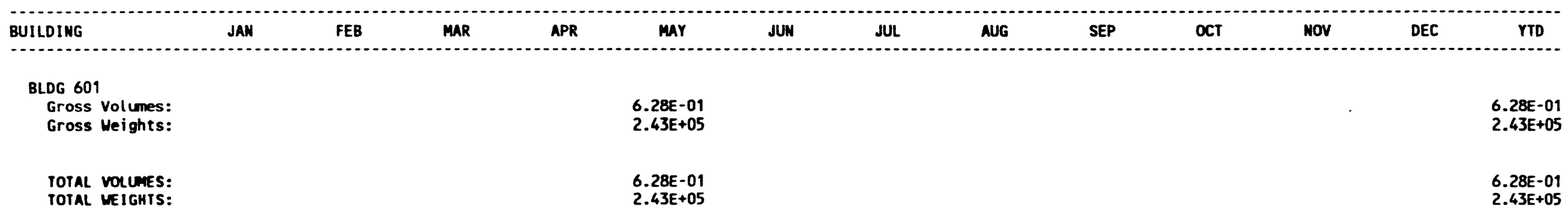


SL1 REACTOR, 1992 DETAIL DATA

Report 10-Hazardous Waste Generated By SLR for January-

December 1992

SLR-3

Report 11-Mixed Waste Generated by SLR For January-

December 1992

SLR-4 
Page: 1

Sched. HO. INRPT013

IDAHO OPERATIONS OFFICE

UNITED STATES DEPARTMENT OF ENERGY

INEL NGERADIOLOGICAL WASTE MAMAGEMENT INFORMATION SYSTEM

HAZARDOUS MASTE GENERATED BY SLR

FOR January - December 1992

RPT 10-1

SLR

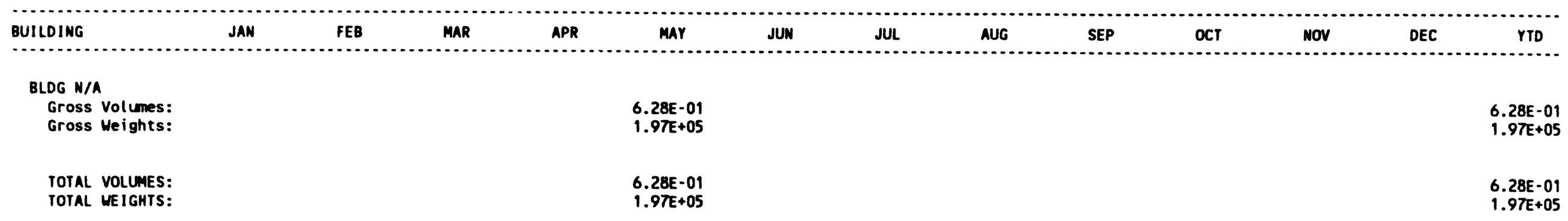

$\underbrace{\infty}_{\omega}$ 
MIXED WASTE GENERATED BY SLR

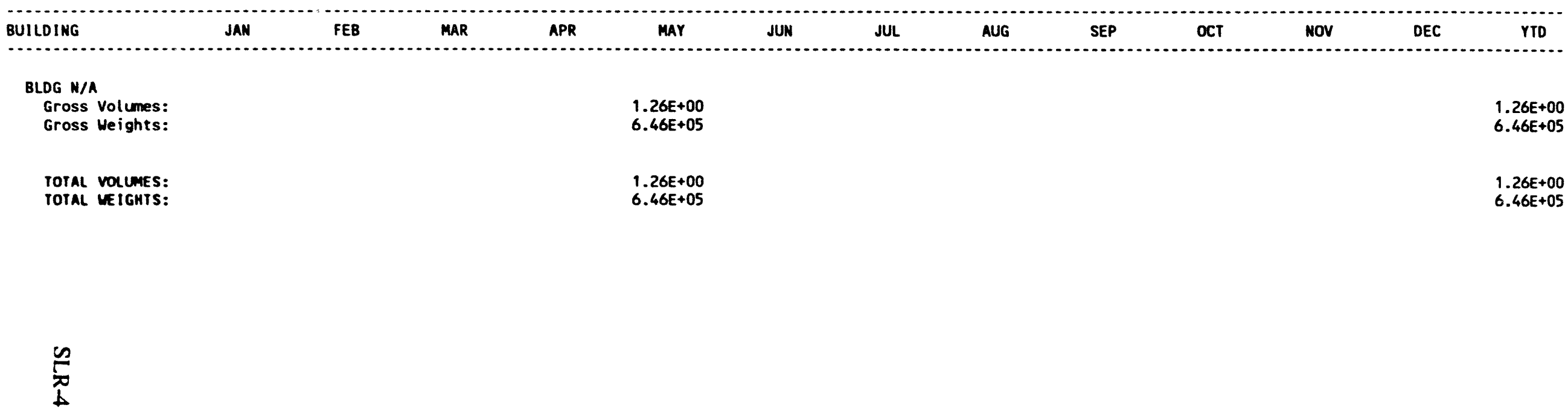


SPECIAL MANUFACTURING CAPABILITY, 1992 DETAIL DATA

Report 278-SMC Fuel Oil Usage And Stack Effluents Summary ......... SMC-3

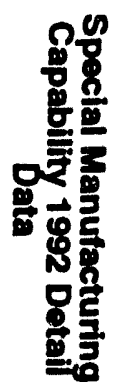


INEL NONRADIOLOGICAL WASTE MANAGEMENT INFORMATION SYSTEM

FOR JANUARY THROUGH DECEMBER 1992

SMC FUEL OIL USAGE AND STACK EFFLUENTS SUMmARY

RPT 278-I

SMC

(ALL VALUES = MEAREST THOUSAMD)

FUEL OIL TYPE
EFFLUENTS
TYPE 2
VOLUME (LITERS)
SO2 (KGS)
NOX (KGS)
PARTICULATES (KGS)

GRAND TOTALS:

VOLUME (LITERS)

SO2 (KGS)

NOX (KGS)

PARTICULATES (KGS) 


\section{TEST AREA NORTH, 1992 DETAIL DATA}

Report 216-TAN Landfill Solid Disposed Waste Summary ............ TAN-3

Report 2110-Hazardous Waste Generated By TAN For January-December $1992 \ldots . \ldots \ldots \ldots \ldots \ldots \ldots \ldots \ldots \ldots \ldots$. . . . . . . . . . . . . . . . . . . . . .

Report 2111-Mixed Waste Generated By TAN For January-December

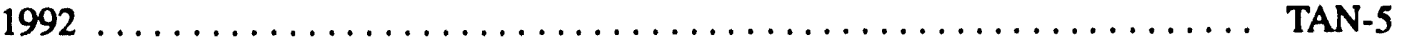


TAN LANDFILL SOL IO DISPOSED MASTE SUMMARY

RPT 216-1

(ALL VOLUMES = CUBIC METERS)

\begin{tabular}{|c|c|c|c|c|c|c|c|c|c|c|c|c|c|}
\hline Type of Haste & JAN & FEB & MAR & APR & Mar & JUN & JUL & AUG & SEP & OCT & Nov & DEC & TOTALS \\
\hline ASBESTOS & & 3.1 & 24.5 & & & & & & & & & & 27.5 \\
\hline ASPHALT & & & & & 0.8 & & 3.8 & 27.5 & 6.9 & 8.0 & & & 47.0 \\
\hline CAFETERIA GARBAGE & & & & 6.1 & & 6.1 & & & & & & & 12.2 \\
\hline DIRT AND GRAVEL & & & 3.8 & & 8.8 & 0.8 & & 11.5 & 81.0 & 45.1 & 19.1 & & 170.1 \\
\hline MASONRY AND CONCRETE & & & & & 3.1 & & 4.2 & 2.3 & 19.1 & 0.4 & 6.9 & & 35.9 \\
\hline SCRAP METAL & 16.1 & 18.4 & 36.7 & 12.6 & 3.1 & 29.1 & 29.4 & 9.9 & 28.7 & 6.9 & 24.5 & 6.1 & 221.4 \\
\hline $\begin{array}{l}\text { TRASH, SWEEPINGS, ETC. } \\
\text { WEEDS, GRASS AND TREES }\end{array}$ & 391.9 & 226.3 & $\begin{array}{r}265.3 \\
3.8 \\
23.7\end{array}$ & 251.9 & 152.9 & 239.7 & $\begin{array}{r}210.3 \\
6.1 \\
270\end{array}$ & 218.7 & $\begin{array}{r}235.9 \\
11.5\end{array}$ & 265.3 & $\begin{array}{r}185.4 \\
1.5 \\
0.2\end{array}$ & 172.0 & $\begin{array}{r}2.815 .6 \\
22.9\end{array}$ \\
\hline $\begin{array}{l}\text { WOOD AND SCRAP LUMBER } \\
\text { AIR DRYER DESICANT }\end{array}$ & 0.4 & 39.8 & 23.7 & & 22.9 & 11.5 & 27.9 & 39.8 & 7.6 & 64.2 & & 0.4 & $\begin{array}{r}247.0 \\
0.4\end{array}$ \\
\hline CRUSHED FLUORESCENT TUB & & & & & & & & & 0.4 & & & & 0.4 \\
\hline ELECTRICAL TRANSFORMERS & 0.4 & & & & & & & & & & & & 0.4 \\
\hline FIBERGLASS & & & & & & & & & 6.1 & & & & 6.1 \\
\hline OILY RAGS & 0.4 & & & & & & & & & 0.8 & & & 1.1 \\
\hline OILY RAGS; FLUORESCENT & & 1.1 & & & & & & & & & & & 1.1 \\
\hline SHREDDED BULBS; SOOIUM & 0.8 & & & & & & & & & & & & 0.8 \\
\hline SHREDDED FLUORESCENT LI & & & & & & 0.8 & & & & & & & 0.8 \\
\hline SHREDLED LIGHT BULBS & & 0.4 & & & & & & & & & & & 0.4 \\
\hline GRAND TOTALS: & 409.8 & 289.0 & 357.8 & 270.7 & 191.5 & 287.9 & 281.8 & 309.7 & 397.2 & 390.7 & 246.6 & 178.5 & $3,611.2$ \\
\hline
\end{tabular}


Page: 1

Sched. No. INRPT013

IDAHO OPERATIONS OFFICE

19-JUN-93 UNITED STATES DEPARTMENT OF ENERGY

RPT 2110-1

HAZARDOUS WASTE GENERATED BY TAN

TAN

FOR January - December 1992

DEC

BUILDING

JAN

FEB

MAR

APR

MAY

JUN

JUL

AUG

SEP

OCT

NOV

DEC

YTD

BLDG 602

Gross Volumes:

Gross Weights:

BLDG 603

Gross Volumes:

Gross Weights:

BLDG 604

$1.14 E-01$

Gross Volumes:

Gross volumes:

$1.72 E+04$

BLDG 607

Gross Volumes: $2.08 \mathrm{E}-0$

Gross Weights: 1.36E+05

BLDG 650

Gross Volumes:

Gross Weights:

$\rightarrow$ BLDG 653

Gross Volumes:

Gross Weights:

TOTAL VOLUMES: $2.08 E-01$

TOTAL WEIGHTS: $1.36 E+05$

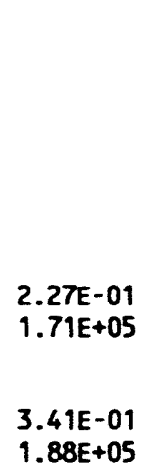

$1.14 E-01$

$1.81 E+04$

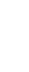

0
$2.27 E-01$
$1.33 E+05$

$1.14 E-01$

$1.81 E+04$
$81 E+04$

1.14E-01

$1.72 E+04$

9.14E+00

$6.65 E+06$

$9.14 E+00$

$6.65 E+06$

2.08E-01

$1.36 \mathrm{E}+05$

2.27E-01

$1.33 E+05$

2.27E-01

$1.71 E+05$

$9.14 E+00$

$6.65 E+06$
$1.00 E+01$

$7.12 E+06$ 
MIXED WASTE GEMERATED BY TAN

FOR January - Decenber 1992

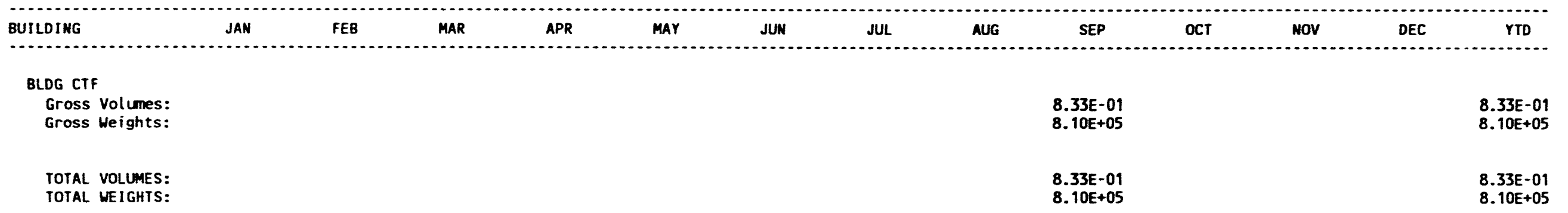

$\sum_{i}^{-1}$

NOTE: VOLUME REPORTED IN CUBIC METERS; WEIGHT REPORTED IN GRAMS DETAILS MAY NOT ADD UP TO TOTALS BECAUSE OF ROWDIMG 


\section{TEST REACTOR AREA, 1992 DETAIL DATA}

Report 235-TRA Liquid Disposed Substances Summary TRA-3

Report 235-TRA Release Point And Liquid Disposed Substances Summary . . TRA-5

Report 236-TRA Landfill Solid Disposed Waste Summary . . . . . . . . . . TRA-9

Report 237-TRA Water Usage And Disposal Summary For January

Through December 1992

Report 238-TRA Fuel Oil Usage And Stack Effluents Summary TRA-12

Report 2310-Hazardous Waste Generated By TRA For January-December 1992

Report 2311-Mixed Waste Generated By TRA For January-December 1992 TRA-14 


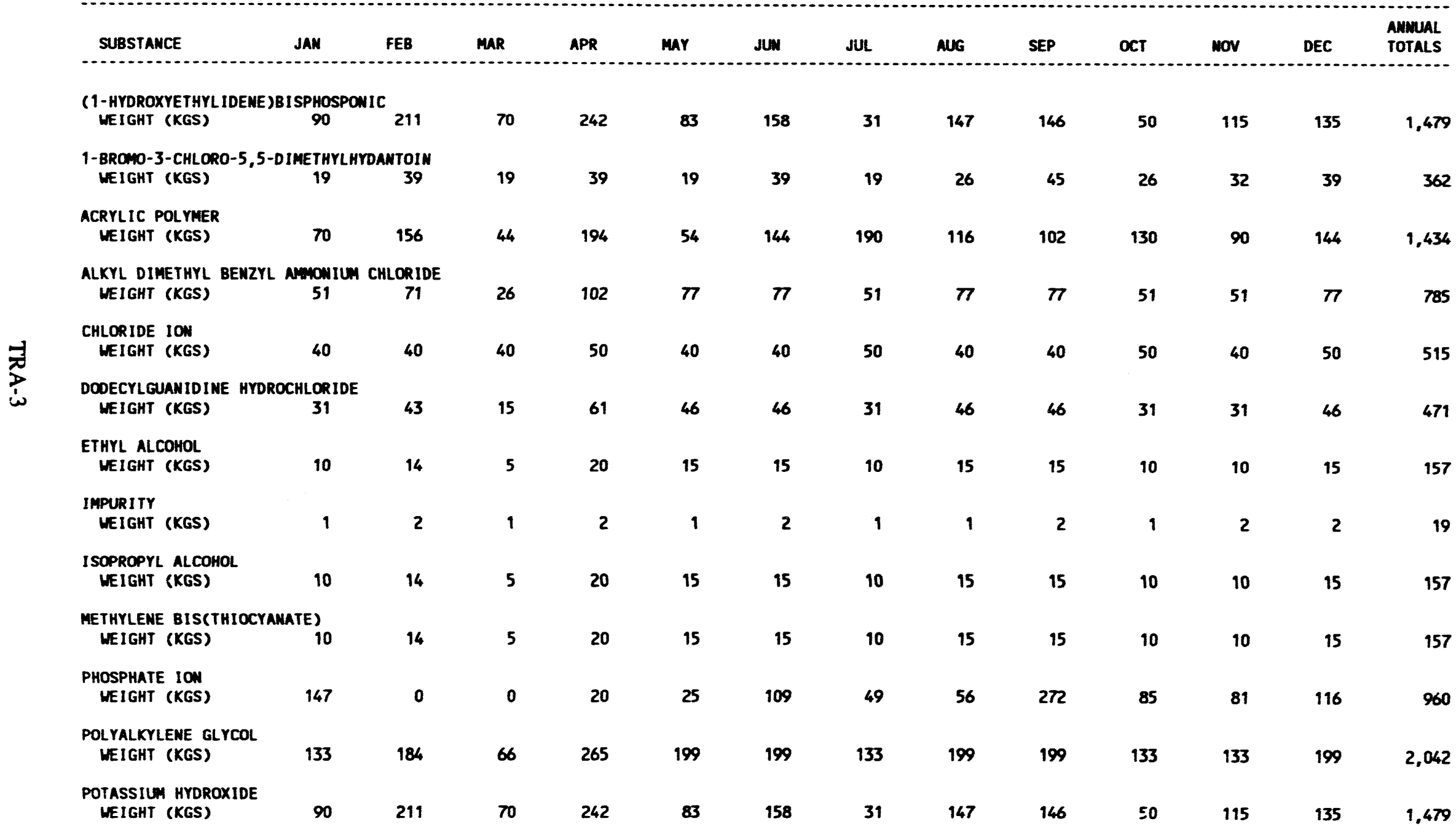


PAGE: 2

SCHED. NO. IMRPTOOSA

IDAHO OPERATIONS OFFICE

$25-54-93$

UNITED STATES DEPARTMEMT OF ENERGY

IWEL WONRADIOLOGICAL MASTE MMMAEEENT IMFORMATION SYSTEM

FOR JAMUARY THROUGH DECEBER 1992

TRA LIOUID DISPOSED SUBSTAMCES SUMARY

RPT 235-1

TRA

\begin{tabular}{|c|c|c|c|c|c|c|c|c|c|c|c|c|c|}
\hline SUBSTAMCE & JAN & FEB & MAR & APR & mar & JuN & JUL & NGG & SEP & OCT & nov & DEC & $\begin{array}{l}\text { AmmuL } \\
\text { TOTALS }\end{array}$ \\
\hline $\begin{array}{l}\text { SILICONE ERULSION } \\
\text { WEIGHT (KGS) }\end{array}$ & 2 & 3 & 1 & 4 & 3 & 3 & 2 & 3 & 3 & 2 & 2 & 3 & 31 \\
\hline $\begin{array}{l}\text { SOOIUM HYDROXIDE } \\
\text { WEIGHT (KGS) }\end{array}$ & 2 & 3 & 1 & 4 & 3 & 3 & 2 & 3 & 3 & 2 & 2 & 3 & 31 \\
\hline $\begin{array}{l}\text { SOOIUM ION } \\
\text { WEIGHT (KGS) }\end{array}$ & 2,430 & 5,941 & 8,697 & 2,311 & 8,837 & 6,028 & 15,653 & 11,543 & 8,138 & 10,461 & 5,120 & 7,186 & 334 \\
\hline $\begin{array}{l}\text { SODIUW TOLYTRIAZOL } \\
\text { WEIGHT (KGS) }\end{array}$ & 90 & 211 & 70 & 242 & 83 & 158 & 31 & 147 & 146 & 50 & 115 & 135 & 1,479 \\
\hline $\begin{array}{l}\text { SULFATE IOW } \\
\text { UEIGHT (KGS) }\end{array}$ & 21,980 & 34,242 & 15,660 & 42,079 & 17,849 & 31,364 & 31,679 & 27,388 & 35,229 & 26,508 & 25,161 & 31,042 & 165,302 \\
\hline $\begin{array}{l}\text { TOS } \\
\text { IEIGHT (KGS) }\end{array}$ & 25.719 & 18,640 & 15,149 & 17,840 & 19,731 & 23,444 & 37,694 & 39.984 & 23,916 & 16,229 & 14,032 & 14.717 & 267,095 \\
\hline $\begin{array}{l}\text { TETRAPOTASSIUN PYR } \\
\text { WEIGHT (KGS) }\end{array}$ & $\begin{array}{l}\text { HOSPHATE } \\
2224\end{array}$ & 527 & 176 & 605 & 207 & 394 & 78 & 369 & 364 & 126 & 288 & 339 & 3,696 \\
\hline $\begin{array}{l}\text { TOTAL HARDNESS } \\
\text { WEIGHT (KGS) }\end{array}$ & 14,807 & 12,202 & 9,112 & 5,048 & 4,949 & 8,957 & 9,484 & 24,380 & 22,181 & 11,148 & 9,239 & 17,400 & 148,906 \\
\hline $\begin{array}{l}\text { TRIBUTYYLTIN OXIDE } \\
\text { WEIGHT (KGS) }\end{array}$ & 10 & 14 & 5 & 20 & 15 & 15 & 10 & 15 & 15 & 10 & 10 & 15 & 157 \\
\hline
\end{tabular}




\begin{tabular}{|c|c|c|c|c|c|c|c|c|c|c|c|c|c|}
\hline $\begin{array}{l}\text { RELEASE POINT DESCRI } \\
\text { SUBSTANCE }\end{array}$ & TION JAN & FEB & MAR & APR & mar & JUW & JUL & AUG & SEP & OCT & NOV & DEC & $\begin{array}{l}\text { ANMUAL TOT } \\
\text { VOL/NEIGHT } \\
\text { AVG CONC }\end{array}$ \\
\hline $\begin{array}{l}\text { CHEMICAL WASTE POND } \\
\text { VOLUME }(1000 \mathrm{~L}) \\
\text { PH }\end{array}$ & $\begin{array}{r}\text { SURFACE } \\
2,305\end{array}$ & 2,040 & 2,623 & 2,828 & 2,146 & 2,358 & 4,262 & 3,312 & 2,729 & 2,990 & 2,093 & 2,616 & 32,305 \\
\hline $\begin{array}{l}\text { SOOIUN ION } \\
\text { CONC. (MG/L) } \\
\text { WEIGHT (KGS) }\end{array}$ & $\begin{array}{r}1042.89 \\
2.404\end{array}$ & $\begin{array}{r}2899.43 \\
5,916\end{array}$ & $\begin{array}{r}3305.71 \\
8,672\end{array}$ & $\begin{array}{r}806.05 \\
2,279\end{array}$ & $\begin{array}{r}4105.08 \\
8,811\end{array}$ & $\begin{array}{r}2544.99 \\
6,002\end{array}$ & $\begin{array}{r}3664.86 \\
15.621\end{array}$ & $\begin{array}{r}3477.29 \\
11.518\end{array}$ & $\begin{array}{r}2972.16 \\
8,112\end{array}$ & $\begin{array}{r}3487.39 \\
10.429\end{array}$ & $\begin{array}{r}2433.55 \\
5,094\end{array}$ & $\begin{array}{r}2734.86 \\
7.154\end{array}$ & $\begin{array}{r}2,848.23 \\
92,011\end{array}$ \\
\hline $\begin{array}{l}\text { SULFATE ION } \\
\text { CONC. (MG/L) } \\
\text { WEIGHT (KGS) } \\
\text { We }\end{array}$ & $\begin{array}{r}6701.49 \\
15.449\end{array}$ & $\begin{array}{r}7123.04 \\
14,533\end{array}$ & $\begin{array}{r}3219.50 \\
8,446\end{array}$ & $\begin{array}{r}5001.61 \\
14.143\end{array}$ & $\begin{array}{r}4635.91 \\
9.950\end{array}$ & $\begin{array}{r}6368.81 \\
15,020\end{array}$ & $\begin{array}{r}5242.27 \\
22,344\end{array}$ & $\begin{array}{r}5031.45 \\
16,665\end{array}$ & $\begin{array}{r}8067.04 \\
22,017\end{array}$ & $\begin{array}{r}3067.28 \\
9.173\end{array}$ & $\begin{array}{r}6024.80 \\
12,612\end{array}$ & $\begin{array}{r}5553.80 \\
14,527\end{array}$ & $\begin{array}{r}5,413.45 \\
174,879\end{array}$ \\
\hline $\begin{array}{l}\text { COLD WASTE POND } \\
\text { VOLUAE }(1000 \mathrm{~L}) \\
\text { PH }\end{array}$ & $\begin{array}{r}\text { SURFACE } \\
36,741\end{array}$ & 28,114 & 22,610 & $\begin{array}{r}28.682 \\
7.9\end{array}$ & $\begin{array}{r}31.722 \\
7.3\end{array}$ & $\begin{array}{r}38,944 \\
7.8\end{array}$ & $\begin{array}{r}60.797 \\
7.3\end{array}$ & $\begin{array}{r}47.431 \\
7.8\end{array}$ & 46,894 & $\begin{array}{r}70,560 \\
8.0\end{array}$ & $\begin{array}{r}57.746 \\
7.8\end{array}$ & $\begin{array}{r}72,498 \\
7.7\end{array}$ & $\begin{array}{r}542.739 \\
7.7\end{array}$ \\
\hline $\begin{array}{l}\text { (1-HYDROXYETHYLIDE } \\
\text { CONC. (MG/L) } \\
\text { WEIGHT (KGS) }\end{array}$ & $\begin{aligned} & \text { E)BISPHOS } \\
& 2.44 \\
& 90\end{aligned}$ & IIC $\begin{array}{r}7.50 \\
211\end{array}$ & $\begin{array}{r}3.11 \\
70\end{array}$ & $\begin{array}{r}8.44 \\
242\end{array}$ & $\begin{array}{r}2.61 \\
83\end{array}$ & $\begin{array}{r}4.05 \\
158\end{array}$ & $\begin{array}{r}0.51 \\
31\end{array}$ & $\begin{array}{r}3.19 \\
147\end{array}$ & $\begin{array}{r}3.11 \\
146\end{array}$ & $\begin{array}{r}0.71 \\
50\end{array}$ & $\begin{array}{r}1.99 \\
115\end{array}$ & $\begin{array}{r}1.87 \\
135\end{array}$ & $\begin{array}{r}2.72 \\
1.479\end{array}$ \\
\hline $\begin{array}{l}\text { 1-BROMO-3-CHLORO-5 } \\
\text { CONC. (MG/L) } \\
\text { MEIGHT (KGS) }\end{array}$ & $\begin{array}{r}\text { 5-DIMETHY } \\
0.53 \\
19\end{array}$ & $\begin{array}{r}\text { TDANTOIN } \\
1.38 \\
39\end{array}$ & $\begin{array}{r}0.86 \\
19\end{array}$ & $\begin{array}{r}1.35 \\
39\end{array}$ & $\begin{array}{r}0.61 \\
19\end{array}$ & $\begin{array}{r}1.00 \\
39\end{array}$ & $\begin{array}{r}0.32 \\
19\end{array}$ & $\begin{array}{r}0.55 \\
26\end{array}$ & $\begin{array}{r}0.96 \\
45\end{array}$ & $\begin{array}{r}0.37 \\
26\end{array}$ & $\begin{array}{r}0.56 \\
32\end{array}$ & $\begin{array}{r}0.53 \\
39\end{array}$ & $\begin{array}{r}0.67 \\
362\end{array}$ \\
\hline $\begin{array}{l}\text { ACRYLIC POL YMER } \\
\text { CONC. (MG/L) } \\
\text { WEIGHT (KGS) }\end{array}$ & $\begin{array}{r}1.90 \\
70\end{array}$ & $\begin{array}{r}5.56 \\
156\end{array}$ & $\begin{array}{r}1.95 \\
44\end{array}$ & $\begin{array}{r}6.76 \\
194\end{array}$ & $\begin{array}{r}1.72 \\
54\end{array}$ & $\begin{array}{r}3.69 \\
144\end{array}$ & $\begin{array}{r}3.12 \\
190\end{array}$ & $\begin{array}{r}2.45 \\
116\end{array}$ & $\begin{array}{r}2.18 \\
102\end{array}$ & $\begin{array}{r}1.85 \\
130\end{array}$ & $\begin{array}{r}1.56 \\
90\end{array}$ & $\begin{array}{r}1.98 \\
144\end{array}$ & $\begin{array}{r}2.64 \\
1.434\end{array}$ \\
\hline $\begin{array}{l}\text { ALKYL DIMETHYL BEN } \\
\text { CONC. (MG/L) } \\
\text { WEIGHT (KGS) }\end{array}$ & $\begin{array}{r}\text { YL AMmONI } \\
1.39 \\
51\end{array}$ & $\begin{array}{r}\text { CHLORIDE } \\
2.52 \\
71\end{array}$ & $\begin{array}{r}1.13 \\
26\end{array}$ & $\begin{array}{r}3.56 \\
102\end{array}$ & $\begin{array}{r}2.41 \\
77\end{array}$ & $\begin{array}{r}1.97 \\
77\end{array}$ & $\begin{array}{r}0.84 \\
51\end{array}$ & $\begin{array}{r}1.61 \\
77\end{array}$ & $\begin{array}{r}1.63 \\
77\end{array}$ & $\begin{array}{r}0.72 \\
51\end{array}$ & $\begin{array}{r}0.88 \\
51\end{array}$ & $\begin{array}{r}1.06 \\
77\end{array}$ & $\begin{array}{r}1.45 \\
785\end{array}$ \\
\hline
\end{tabular}




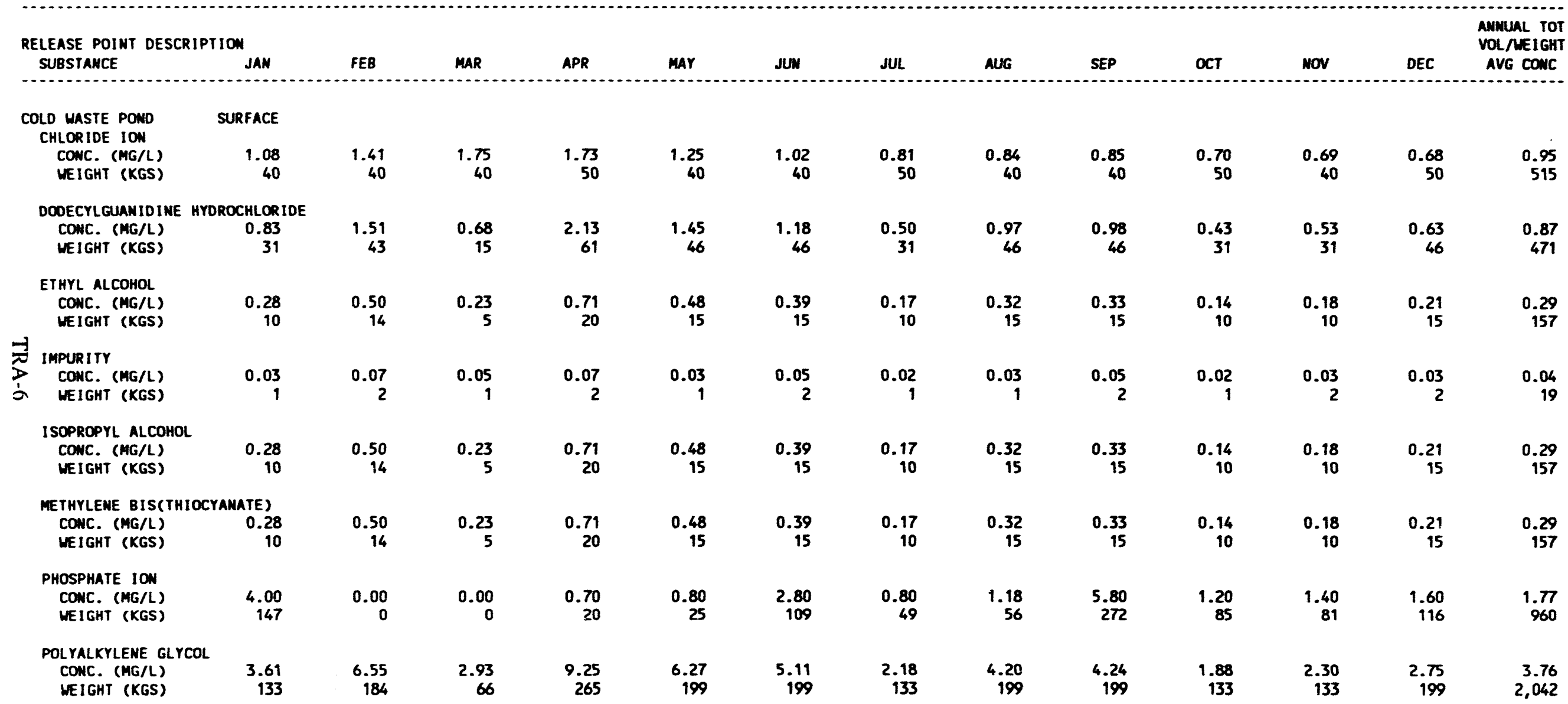




\begin{tabular}{|c|c|c|c|c|c|c|c|c|c|c|c|c|c|}
\hline $\begin{array}{l}\text { RELEASE POINT DES } \\
\text { SUBSTANCE }\end{array}$ & TION JAN & FEB & MAR & APR & MAY & JUN & JUL & AUG & SEP & OCT & NOV & DEC & $\begin{array}{l}\text { ANNUAL TOT } \\
\text { VOL/MEIGHT } \\
\text { AVG CONC }\end{array}$ \\
\hline $\begin{array}{l}\text { COLD WASTE POND } \\
\text { POTASSIUM HYDRG }\end{array}$ & SURFACE & & & & & & & & & & & & \\
\hline $\begin{array}{l}\text { CONC. (MG/L) } \\
\text { WEIGHT (KGS) }\end{array}$ & $\begin{array}{r}2.44 \\
90\end{array}$ & $\begin{array}{r}7.50 \\
211\end{array}$ & $\begin{array}{r}3.11 \\
70\end{array}$ & $\begin{array}{r}8.44 \\
242\end{array}$ & $\begin{array}{r}2.61 \\
83\end{array}$ & $\begin{array}{r}4.05 \\
158\end{array}$ & $\begin{array}{r}0.51 \\
31\end{array}$ & $\begin{array}{r}3.11 \\
147\end{array}$ & $\begin{array}{r}3.11 \\
146\end{array}$ & $\begin{array}{r}0.71 \\
50\end{array}$ & $\begin{array}{r}1.99 \\
115\end{array}$ & $\begin{array}{r}1.87 \\
135\end{array}$ & $\begin{array}{r}2.72 \\
1.479\end{array}$ \\
\hline $\begin{array}{l}\text { SILICONE EMULSI } \\
\text { CONC. (MG/L) } \\
\text { WEIGHT (KGS) }\end{array}$ & $\begin{array}{r}0.06 \\
2\end{array}$ & $\begin{array}{r}0.10 \\
3\end{array}$ & $\begin{array}{r}0.05 \\
1\end{array}$ & $\begin{array}{r}0.14 \\
4\end{array}$ & $\begin{array}{r}0.10 \\
3\end{array}$ & $\begin{array}{r}0.08 \\
3\end{array}$ & $\begin{array}{r}0.03 \\
2\end{array}$ & $\begin{array}{r}0.06 \\
3\end{array}$ & $\begin{array}{r}0.07 \\
3\end{array}$ & $\begin{array}{r}0.03 \\
2\end{array}$ & $\begin{array}{r}0.04 \\
2\end{array}$ & $\begin{array}{r}0.04 \\
3\end{array}$ & $\begin{array}{r}0.06 \\
31\end{array}$ \\
\hline $\begin{array}{l}\text { SOOIUM HYDROXID } \\
\text { CONC. (MG/L) } \\
\text { WEIGHT (KGS) }\end{array}$ & $\begin{array}{r}0.06 \\
2\end{array}$ & $\begin{array}{r}0.10 \\
3\end{array}$ & $\begin{array}{r}0.05 \\
1\end{array}$ & $\begin{array}{r}0.14 \\
4\end{array}$ & $\begin{array}{r}0.10 \\
3\end{array}$ & $\begin{array}{r}0.08 \\
3\end{array}$ & $\begin{array}{r}0.03 \\
2\end{array}$ & $\begin{array}{r}0.06 \\
3\end{array}$ & $\begin{array}{r}0.07 \\
3\end{array}$ & $\begin{array}{r}0.03 \\
2\end{array}$ & $\begin{array}{r}0.04 \\
2\end{array}$ & $\begin{array}{r}0.04 \\
3\end{array}$ & $\begin{array}{r}0.06 \\
31\end{array}$ \\
\hline $\begin{array}{l}\text { SOOIUM ION } \\
\text { CONC. (MG/L) } \\
\text { WEIGHT (KGS) }\end{array}$ & $\begin{array}{r}0.70 \\
26\end{array}$ & $\begin{array}{r}0.91 \\
26\end{array}$ & $\begin{array}{r}1.14 \\
26\end{array}$ & $\begin{array}{r}1.12 \\
32\end{array}$ & $\begin{array}{r}0.81 \\
26\end{array}$ & $\begin{array}{r}0.66 \\
26\end{array}$ & $\begin{array}{r}0.53 \\
32\end{array}$ & $\begin{array}{r}0.54 \\
26\end{array}$ & $\begin{array}{r}0.55 \\
26\end{array}$ & $\begin{array}{r}0.45 \\
32\end{array}$ & $\begin{array}{r}0.44 \\
26\end{array}$ & $\begin{array}{r}0.44 \\
32\end{array}$ & $\begin{array}{r}0.61 \\
334\end{array}$ \\
\hline $\begin{array}{l}\text { SOOIUN TOLYTRIA } \\
\text { CONC. (MG/L) } \\
\text { WEIGHT (KGS) }\end{array}$ & $\begin{array}{r}2.44 \\
90\end{array}$ & $\begin{array}{r}7.50 \\
211\end{array}$ & $\begin{array}{r}3.11 \\
70\end{array}$ & $\begin{array}{r}8.44 \\
242\end{array}$ & $\begin{array}{r}2.61 \\
83\end{array}$ & $\begin{array}{r}4.05 \\
158\end{array}$ & $\begin{array}{r}0.51 \\
31\end{array}$ & $\begin{array}{r}3.11 \\
147\end{array}$ & $\begin{array}{r}3.11 \\
146\end{array}$ & $\begin{array}{r}0.71 \\
50\end{array}$ & $\begin{array}{r}1.99 \\
115\end{array}$ & $\begin{array}{r}1.87 \\
135\end{array}$ & $\begin{array}{r}2.72 \\
1.479\end{array}$ \\
\hline $\begin{array}{l}\text { SULFATE ION } \\
\text { CONC. (MG/L) } \\
\text { WEIGHT (KGS) }\end{array}$ & $\begin{array}{r}177.75 \\
6.531\end{array}$ & $\begin{array}{l}701.02 \\
19.708\end{array}$ & $\begin{array}{r}319.09 \\
7.215\end{array}$ & $\begin{array}{l}973.98 \\
27,936\end{array}$ & $\begin{array}{r}249.00 \\
7,899\end{array}$ & $\begin{array}{l}419.68 \\
16.344\end{array}$ & $\begin{array}{r}153.54 \\
9.335\end{array}$ & $\begin{array}{l}226.07 \\
10.723\end{array}$ & $\begin{array}{l}281.74 \\
13,212\end{array}$ & $\begin{array}{l}245.69 \\
17,336\end{array}$ & $\begin{array}{l}217.31 \\
12,549\end{array}$ & $\begin{array}{l}227.80 \\
16,515\end{array}$ & $\begin{array}{r}304.57 \\
165.302\end{array}$ \\
\hline $\begin{array}{l}\text { TDS } \\
\text { CONC. (MG/L) } \\
\text { WEIGHT (KGS) }\end{array}$ & $\begin{array}{l}700.00 \\
25,719\end{array}$ & $\begin{array}{l}663.00 \\
18,640\end{array}$ & $\begin{array}{l}670.00 \\
15,149\end{array}$ & $\begin{array}{l}622.00 \\
17,840\end{array}$ & $\begin{array}{l}622.00 \\
19.731\end{array}$ & $\begin{array}{l}602.00 \\
23,444\end{array}$ & $\begin{array}{l}620.00 \\
37,694\end{array}$ & $\begin{array}{l}843.00 \\
39,984\end{array}$ & $\begin{array}{l}510.00 \\
23,916\end{array}$ & $\begin{array}{l}230.00 \\
16,229\end{array}$ & $\begin{array}{l}243.00 \\
14,032\end{array}$ & $\begin{array}{l}203.00 \\
14,717\end{array}$ & $\begin{array}{r}492.12 \\
267,095\end{array}$ \\
\hline $\begin{array}{l}\text { TETRAPOTASSIUM } \\
\text { CONC. (MG/L) } \\
\text { WEIGHT (KGS) }\end{array}$ & $\begin{array}{c}\text { PPOSPHATE } \\
6.10 \\
224\end{array}$ & $\begin{array}{r}18.76 \\
527\end{array}$ & $\begin{array}{r}7.77 \\
176\end{array}$ & $\begin{array}{r}21.10 \\
605\end{array}$ & $\begin{array}{r}6.52 \\
207\end{array}$ & $\begin{array}{r}10.12 \\
394\end{array}$ & $\begin{array}{r}1.28 \\
78\end{array}$ & $\begin{array}{r}7.77 \\
369\end{array}$ & $\begin{array}{c}7.77 \\
364\end{array}$ & $\begin{array}{r}1.79 \\
126\end{array}$ & $\begin{array}{r}4.98 \\
288\end{array}$ & $\begin{array}{r}4.67 \\
339\end{array}$ & $\begin{array}{r}6.81 \\
3,696\end{array}$ \\
\hline
\end{tabular}


PAGE: 4

SCHED. NO. INRPTOOS
IDAHO OPERATIONS OFFICE

UNITED STATES DEPARTMENT OF EMERGY

IMEL MOMRADIOLOGICAL WASTE MAMAGEMENT INFORMATION SYSTEM

FOR JAMUARY THROUGH DECEMBER 1992

TRA RELEASE POINT AND LIQUID DISPOSED SUBSTANCES SUMMARY

RPT 235-I

TRA

\begin{tabular}{|c|c|c|c|c|c|c|c|c|c|c|c|c|c|}
\hline $\begin{array}{l}\text { RELEASE POINT DESCRI } \\
\text { SUBSTANCE }\end{array}$ & ${ }_{\text {TION }}^{\text {JAN }}$ & FEB & MAR & APR & MAY & JUN & JUL & AUG & SEP & OCT & Nov & DEC & $\begin{array}{l}\text { ANMUAL TOT } \\
\text { VOL/MEIGHT } \\
\text { AVG CONC }\end{array}$ \\
\hline $\begin{array}{l}\text { COLD WASTE POND } \\
\text { TOTAL HARDNESS }\end{array}$ & SURFACE & & & & & & & & & & & & \\
\hline $\begin{array}{l}\text { CONC. (MG/L) } \\
\text { WEIGHT (KGS) }\end{array}$ & $\begin{array}{l}403.00 \\
14.807\end{array}$ & $\begin{array}{l}434.00 \\
12,202\end{array}$ & $\begin{array}{r}403.00 \\
9.112\end{array}$ & $\begin{array}{r}176.00 \\
5,048\end{array}$ & $\begin{array}{r}156.00 \\
4.949\end{array}$ & $\begin{array}{r}230.00 \\
8,957\end{array}$ & $\begin{array}{r}156.00 \\
9.484\end{array}$ & $\begin{array}{l}514.00 \\
24,380\end{array}$ & $\begin{array}{l}473.00 \\
22.181\end{array}$ & $\begin{array}{l}158.00 \\
11,148\end{array}$ & $\begin{array}{r}160.00 \\
9,239\end{array}$ & $\begin{array}{l}240.00 \\
17,400\end{array}$ & $\begin{array}{r}274.36 \\
148,906\end{array}$ \\
\hline $\begin{array}{l}\text { TRIBUTYLTIN OXIDE } \\
\text { CONC. (MG/L) } \\
\text { CEIGHT (KGS) }\end{array}$ & $\begin{array}{r}0.28 \\
10\end{array}$ & $\begin{array}{r}0.50 \\
14\end{array}$ & $\begin{array}{r}0.23 \\
5\end{array}$ & $\begin{array}{r}0.71 \\
20\end{array}$ & $\begin{array}{r}0.48 \\
15\end{array}$ & $\begin{array}{r}0.39 \\
15\end{array}$ & $\begin{array}{r}0.17 \\
10\end{array}$ & $\begin{array}{r}0.32 \\
15\end{array}$ & $\begin{array}{r}0.33 \\
15\end{array}$ & $\begin{array}{r}0.14 \\
10\end{array}$ & $\begin{array}{r}0.18 \\
10\end{array}$ & $\begin{array}{r}0.21 \\
15\end{array}$ & $\begin{array}{r}0.29 \\
157\end{array}$ \\
\hline $\begin{array}{l}\text { COOL ING TONERS EVAP } \\
\text { VOLUME }(1000 \mathrm{~L}) \\
\text { PH }\end{array}$ & $\begin{array}{l}\text { AIRBORNE } \\
58,291\end{array}$ & 117,858 & 94,480 & 145,836 & 87,719 & 87,719 & 67,717 & 74,122 & 72,858 & 73,210 & 73,130 & 95,570 & $1,048,510$ \\
\hline $\begin{array}{l}\text { IRRIGATION } \\
\text { VOLUME }(1000 \mathrm{~L}) \\
\text { PH }\end{array}$ & $\begin{array}{r}\text { SURFACE } \\
4,380\end{array}$ & 49,721 & 15,687 & 46,114 & 30,722 & 87,098 & 68,947 & 42,525 & 35,511 & 43,456 & 23,859 & 25,657 & 473,678 \\
\hline $\begin{array}{l}\text { RADIOACTIVE STREAM } \\
\text { VOLUME }(1000 \mathrm{~L}) \\
\text { PH }\end{array}$ & $\begin{array}{r}\text { SURFACE } \\
8,264\end{array}$ & 5,674 & 11,795 & $\begin{array}{r}6.424 \\
6.2\end{array}$ & $\begin{array}{r}5.224 \\
6.1\end{array}$ & 6,083 & 9,702 & 6,409 & 6,147 & 9,066 & 6,799 & 6,889 & $\begin{array}{r}88,476 \\
6.2\end{array}$ \\
\hline $\begin{array}{l}\text { SEWAGE PLANT } \\
\text { VOLUME }(1000 \mathrm{~L}) \\
\text { PH }\end{array}$ & $\begin{array}{r}\text { SURFACE } \\
4,732\end{array}$ & $\begin{array}{r}3,729 \\
7.6\end{array}$ & $\begin{array}{r}4.349 \\
7.5\end{array}$ & $\begin{array}{r}3,494 \\
7.6\end{array}$ & $\begin{array}{r}3.184 \\
8.1\end{array}$ & $\begin{array}{r}3,456 \\
7.1\end{array}$ & $\begin{array}{r}3,808 \\
7.2\end{array}$ & $\begin{array}{r}4.770 \\
7.1\end{array}$ & $\begin{array}{r}3,891 \\
7.2\end{array}$ & 4,395 & $\begin{array}{r}3,664 \\
7.6\end{array}$ & $\begin{array}{r}3,982 \\
7.6\end{array}$ & $\begin{array}{r}47.454 \\
7.5\end{array}$ \\
\hline
\end{tabular}


INEL NONRADIOLOGICAL WASTE MANAGEMENT INFORMATION SYSTEM

FOR JANUARY THROUGH DECEMBER 1992

TRA LANDFILL SOLID DISPOSED MASTE SUMMARY

RPT 236-1

(ALL VOLUMES = CUBIC METERS)

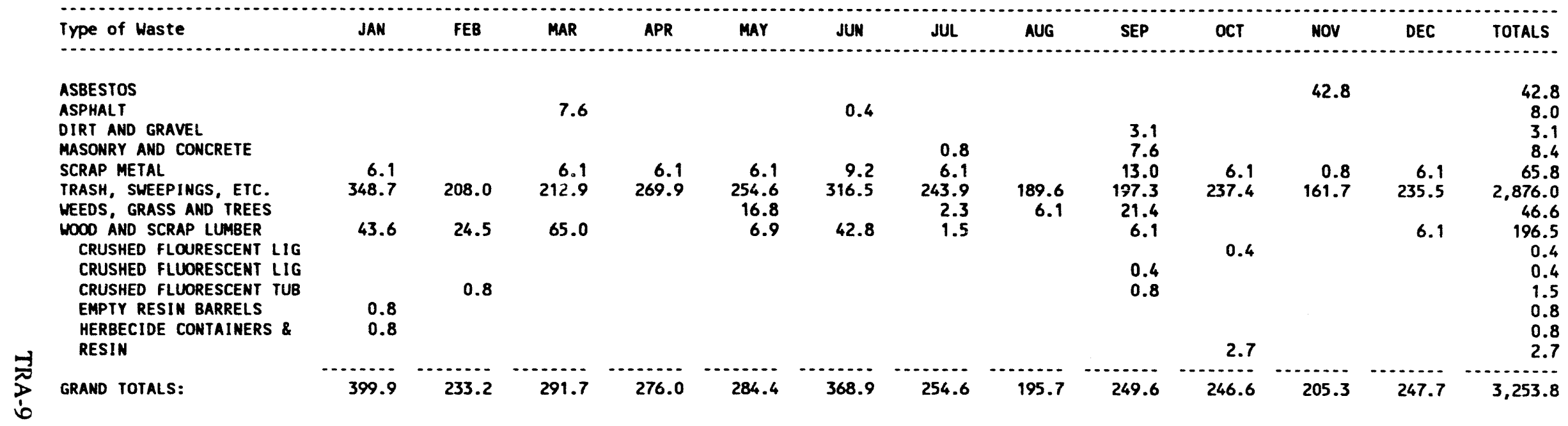


OLOGICAL WASTE MANAGEMENT INFORMATION SYSTEH

FOR JANUARY THROUGH DECEMBER 1992

IRA WATER USAGE AND DISPOSAL SUMMARY

RPT 237-I

(ALL VOLUMES = NEAREST THOUSAND LITERS)

ANNUAL TOT

VOL/WEIGHT

WELL OR BLDG. NO./

JAN

FEB

MAR APR MAY

JUN

JUL

AUG

DEC AVG CONC

WATER DATA

001 MATER PUMPED (L)

(L)

$32.657 \quad 4,202$

004 WATER PUIPED (L)

MATER PUMPED (L)

$\begin{array}{rrrrrrrr}82,067 & 202,935 & 151,545 & 233,377 & 160,936 & 167,330 & 87,197 & 112,548\end{array}$

$\begin{array}{rrrrrr}57,432 & 114,558 & 2,048 & 58,424 & 269,320 \\ & 9,217 & 60,661 & & 69,878\end{array}$ $\begin{array}{lrrrr}168,030 & 203,541 & 167,292 & 148,789 & 1,885,587\end{array}$

WATER OISPOSED

AIR (L)
SURFACE (L)
SUBSURFACE (L)

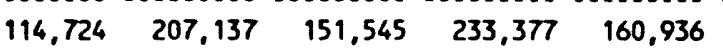

$224,762 \quad 210,972$

175,256

$168,030 \quad 203,54$

$207,2132,224,786$

SEWAGE DATA

\begin{tabular}{|c|c|c|c|c|c|c|c|c|c|c|c|c|}
\hline BUILD VOL (L) & 3,729 & 4,349 & 3,494 & 3,184 & 3,456 & 3,808 & 4,770 & 3,891 & 4,395 & 3,664 & 3,982 & 47,454 \\
\hline $\begin{array}{l}\text { BIOCHEMICAL OXYGEN DEMAND (BOD) } \\
\text { RAW CONC. (MG/L) } \\
\text { FINAL CONC. (MG/L) } \\
\chi \text { REMOVED }\end{array}$ & $\begin{array}{r}199.0 \\
24.8 \\
87.5\end{array}$ & $\begin{array}{r}230.0 \\
26.0 \\
88.7\end{array}$ & $\begin{array}{r}107.5 \\
23.5 \\
78.1\end{array}$ & $\begin{array}{r}188.3 \\
27.7 \\
85.3\end{array}$ & $\begin{array}{r}105.0 \\
25.5 \\
75.7\end{array}$ & $\begin{array}{r}158.8 \\
20.5 \\
87.1\end{array}$ & $\begin{array}{r}190.0 \\
22.8 \\
88.0\end{array}$ & $\begin{array}{r}116.3 \\
58.5 \\
49.7\end{array}$ & $\begin{array}{r}140.0 \\
16.0 \\
88.6\end{array}$ & $\begin{array}{r}28.0 \\
86.0 \\
207.1\end{array}$ & $\begin{array}{r}135.0 \\
18.5 \\
86.3\end{array}$ & $\begin{array}{r}145.3 \\
31.8 \\
78.1\end{array}$ \\
\hline $\begin{array}{l}\text { DISSOLVED OXYGEN (DO) } \\
\text { RAW CONC. (MG/L) } \\
\text { FINAL CONC. (MG/L) } \\
\text { INCREASED FACTOR }\end{array}$ & $\begin{array}{l}2.3 \\
5.2 \\
1.3\end{array}$ & $\begin{array}{l}2.7 \\
4.6 \\
0.7\end{array}$ & $\begin{array}{l}2.3 \\
5.0 \\
1.2\end{array}$ & $\begin{array}{l}2.1 \\
4.4 \\
1.1\end{array}$ & $\begin{array}{l}2.5 \\
3.8 \\
0.5\end{array}$ & $\begin{array}{l}2.0 \\
4.2 \\
1.1\end{array}$ & $\begin{array}{l}2.5 \\
3.7 \\
0.5\end{array}$ & $\begin{array}{l}2.9 \\
5.2 \\
0.8\end{array}$ & $\begin{array}{l}3.3 \\
5.0 \\
0.5\end{array}$ & $\begin{array}{l}3.7 \\
5.8 \\
0.6\end{array}$ & $\begin{array}{l}3.7 \\
5.9 \\
0.6\end{array}$ & $\begin{array}{l}2.7 \\
4.8 \\
0.8\end{array}$ \\
\hline $\begin{array}{l}\text { SETTLEABLE SOLIDS (SS) } \\
\text { RAW CONC. (ML/L) } \\
\text { FINAL CONC. (ML/L) } \\
\text { X REMOVED }\end{array}$ & $\begin{array}{r}4.8 \\
0.01 \\
99.8\end{array}$ & $\begin{array}{r}3.8 \\
0.20 \\
94.7\end{array}$ & $\begin{array}{r}4.1 \\
0.15 \\
96.3\end{array}$ & $\begin{array}{r}6.6 \\
0.13 \\
98.0\end{array}$ & $\begin{array}{r}6.0 \\
0.11 \\
98.2\end{array}$ & $\begin{array}{r}5.1 \\
0.14 \\
97.3\end{array}$ & $\begin{array}{r}5.7 \\
0.11 \\
98.1\end{array}$ & $\begin{array}{r}5.1 \\
0.11 \\
97.8\end{array}$ & $\begin{array}{r}4.7 \\
0.09 \\
98.1\end{array}$ & $\begin{array}{r}5.7 \\
0.09 \\
98.4\end{array}$ & $\begin{array}{r}5.5 \\
0.05 \\
99.1\end{array}$ & $\begin{array}{r}5.2 \\
0.11 \\
97.9\end{array}$ \\
\hline PH FINAL & 7.6 & 7.5 & 7.5 & 8.1 & 7.1 & 7.2 & 7.1 & 7.2 & 7.6 & 7.6 & 7.6 & 7.5 \\
\hline TOTAL SEWAGE(L) & 3,729 & 4,349 & 3,494 & 3,184 & $3,4.56$ & 3,808 & 4,770 & 3,891 & 4,395 & 3,664 & 3,982 & 47,454 \\
\hline
\end{tabular}

DETAILS MAY NOT ADD UP TO TOTALS BECAUSE OF ROUNDING

O IN A COLUMN IMOICATES A TOTAL OF $<0.5$

0.0 IN A COLUMM IMDICATES $A$ TOTAL OF $<0.05$

0.00 IN A COLLHN INDICATES A TOTAL OF $<0.005$ 
PAGE: 2

SCHED. NO. INRPT010
IDAHO OPERATIONS OFFICE

UNITED STATES DEPARTMENT OF ENERGY

INEL NONRADIOLOGICAL WASTE MANAGEMENT INFORMATION SYSTEM

FOR JAMUARY THROUGH DECEMBER 1992

IRA HATER USAGE AND DISPOSAL SUMMARY

(ALL VOLUMES = MEAREST THOUSAND LITERS)

RPT 237-I

TRA

ANMUAL TOT

VOLANEIGHT

WELL OR BLDG. NO./

SEMAGE BLDG. NO.

JAN

FEB MAR

APR

MaY

JUN

JUL DEC AVG CONC

TOTAL WATER DISPOSED(L) 114,698

207,137

ACCONTABILITY $(x)$

100.0

100.0

100.0

233,377

164,120

228,218

214,780

180,026

171,922

207,936

$219,195 \quad 2,255,909$

102.3

102.2

102.2

101.9

101.4

DETAILS MAY NOT ADD UP TO TOTALS BECAUSE OF ROUNDING

0 IN A COLUAN INDICATES A TOTAL OF $<0.5$

0.00 IN $A$ COLUMN INDICATES $\hat{A}$ TOTAL OF $<0.005$ 
PAGE: 1

IDAHO OPERATIONS OFFICE

19- JUN-93

SCHED. NO. INRPTO11

UNITED STATES DEPARTMENT OF ENERGY

INEL NONRADIOLOGICAL WASTE MANAGEMENT INFORMATION SYSTEM

FOR JANUARY THROUGH DECEMBER 1992

TRA FUEL OIL USAGE AND STACK EFFLUENTS SUMMARY

RPT 238-1

(ALL VALUES $=$ MEAREST THOUSAND)

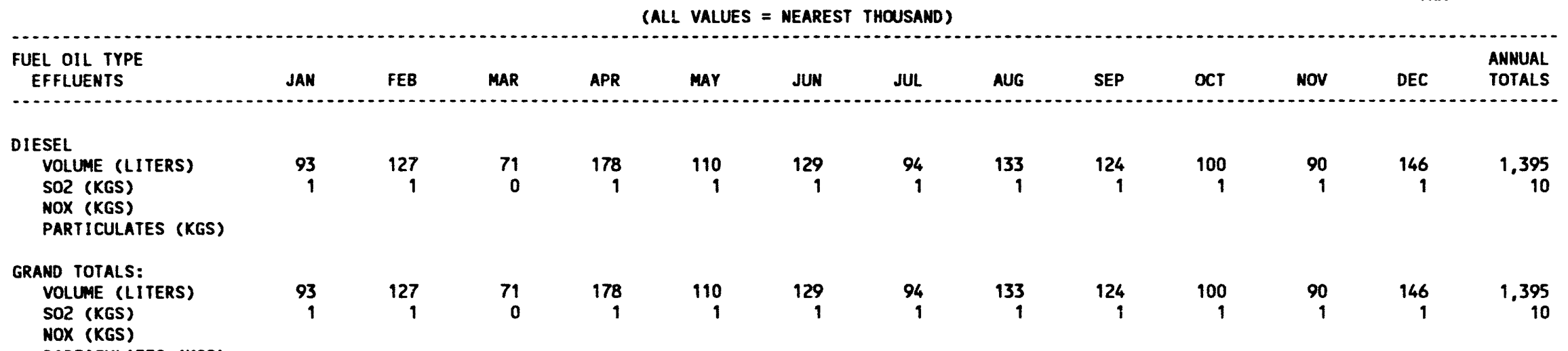

$\frac{1}{0}$

O IN A COLUNM INDICATES A TOTAL OF $<0.5$ 
INEL NONRADIOLOGICAL WASTE MANAGEMENT INFORMATION SYSTEM

HAZARDOUS WASTE GENERATED BY TRA
FOR JanuarY - December 1992

RPT 2310-1

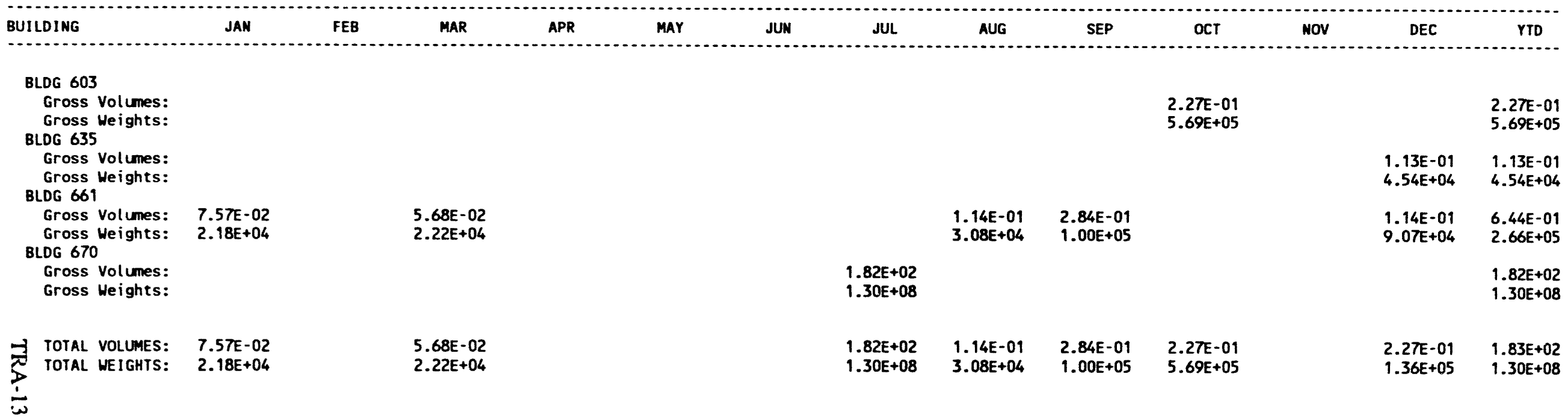

NOTE: VOLUME REPORTED IN CUBIC METERS: WEIGHT REPORTED IN GRAMS DETAILS MAY NOT ADD UP TO TOTALS BECAUSE OF ROUNDING 
MIXED WASTE GENERATED BY TRA

FOR January - December 1992

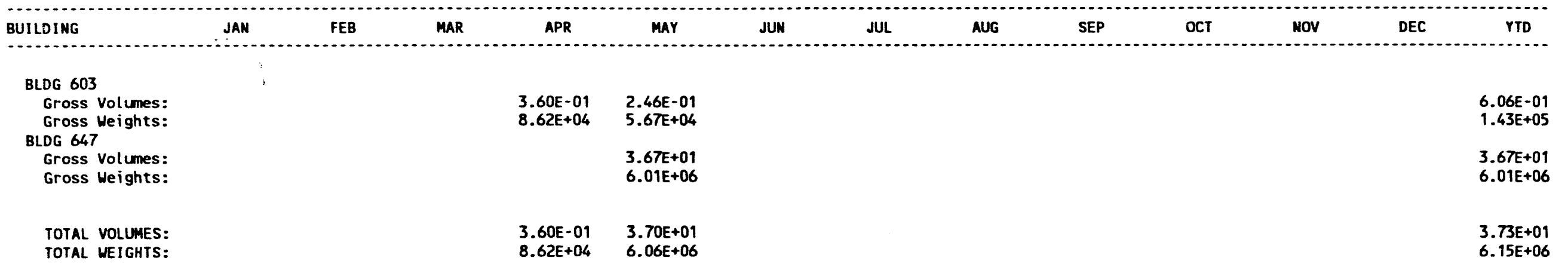

$\frac{\text { 궁 }}{\stackrel{3}{1}}$ 
TECHNICAL SUPPORT FACILITY_TEST AREA NORTH, 1992 DETAIL DATA

Report 10-TSF Hazardous Waste Generated By TSB For JanuaryDecember 1992

TSB-3 
TECHNICAL SUPPORT FACILITY-TEST AREA NORTH, 1992 DETAIL DATA

Report 225-TSF Liquid Disposed Substances Summary $\ldots \ldots \ldots \ldots \ldots$ TSF-3

Report 225-TSF Release Point And Liquid Disposed Substances Summary . . . TSF-5

Report 227-TSF Water Usage And Disposal Summary For January

Through December $1992 \ldots \ldots \ldots \ldots \ldots \ldots \ldots \ldots \ldots \ldots \ldots \ldots$ TSF-6

Report 228-TSF Fuel Oil Usage And Stack Effluents Summary . . . . . . . . . TSF-8 


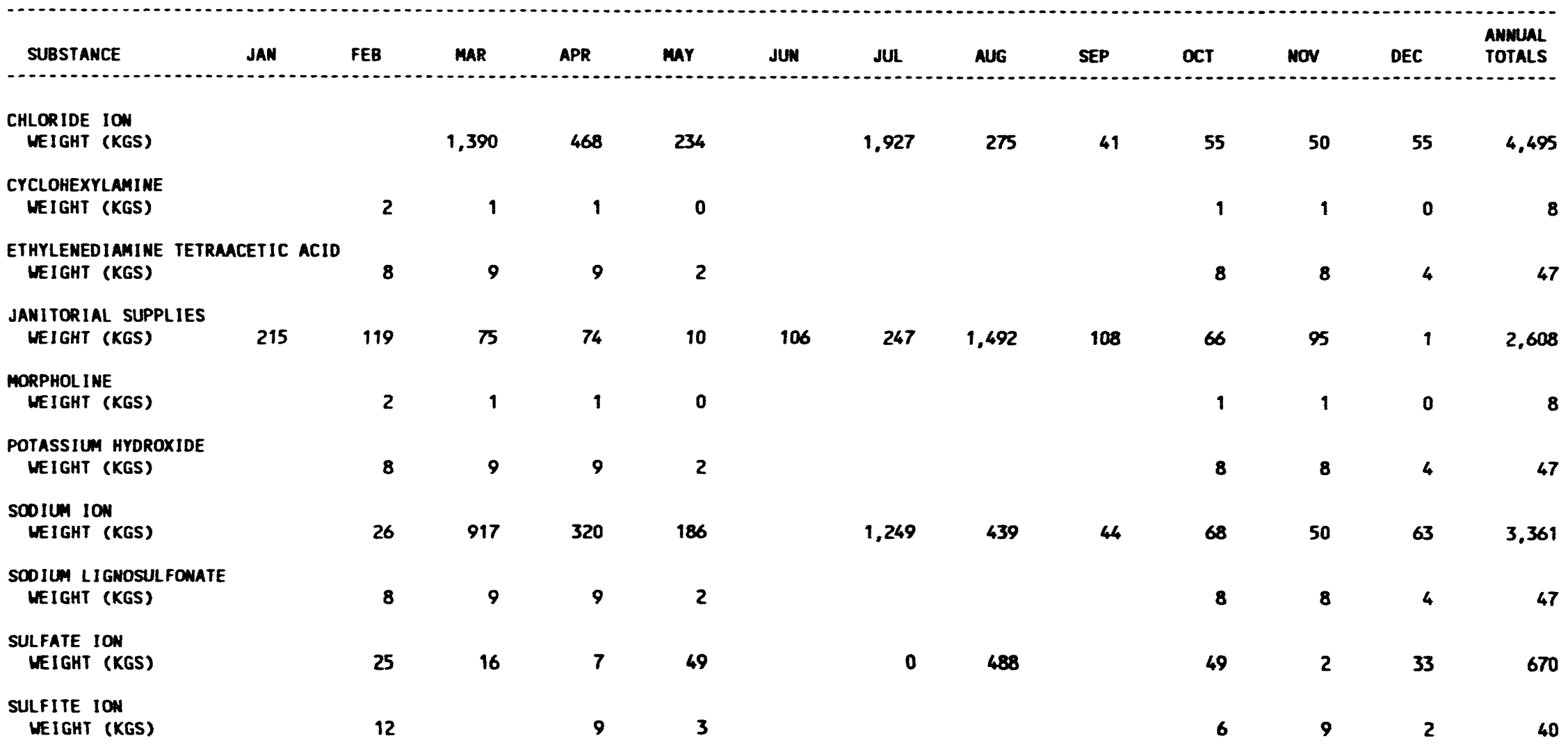




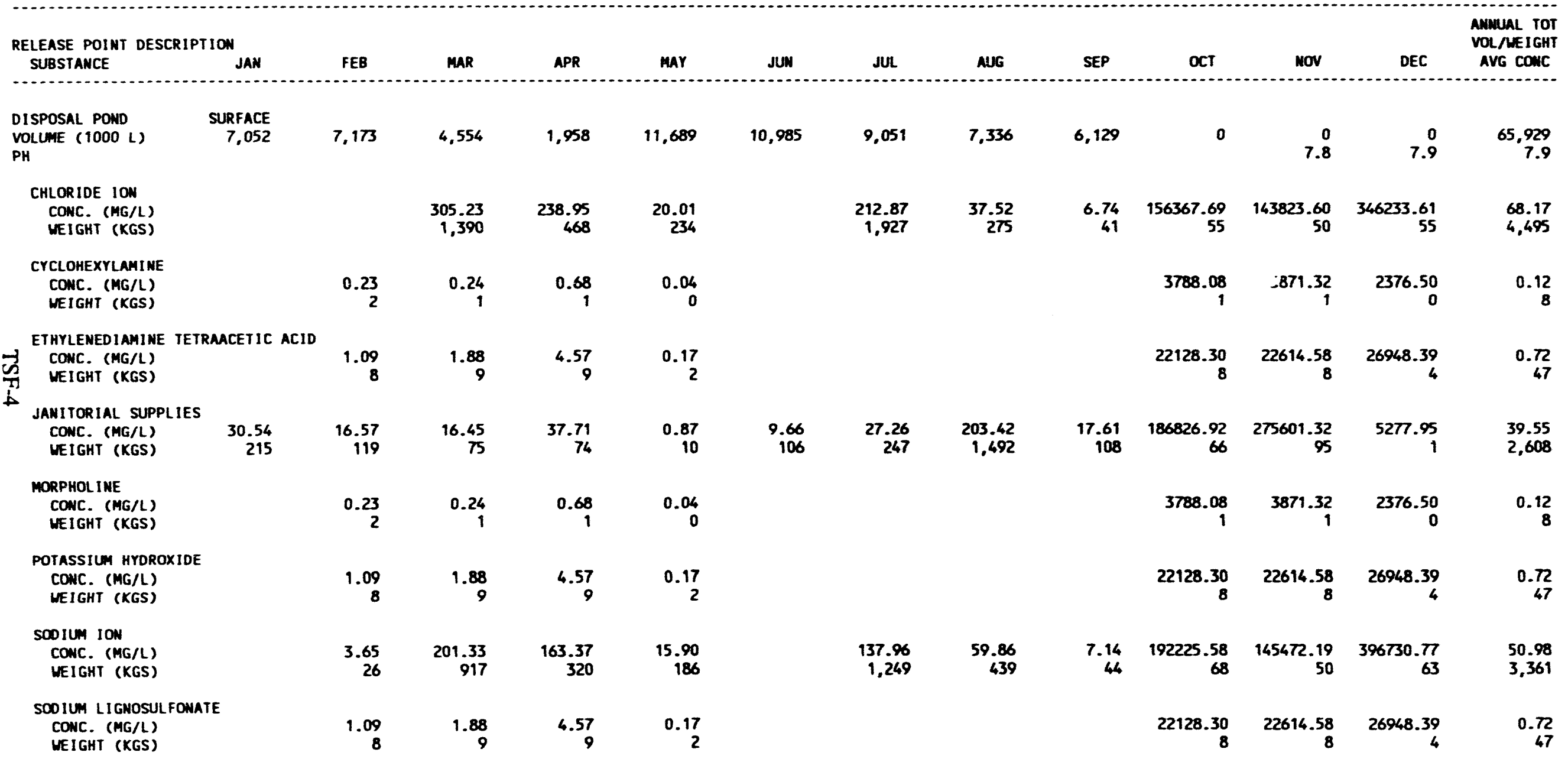




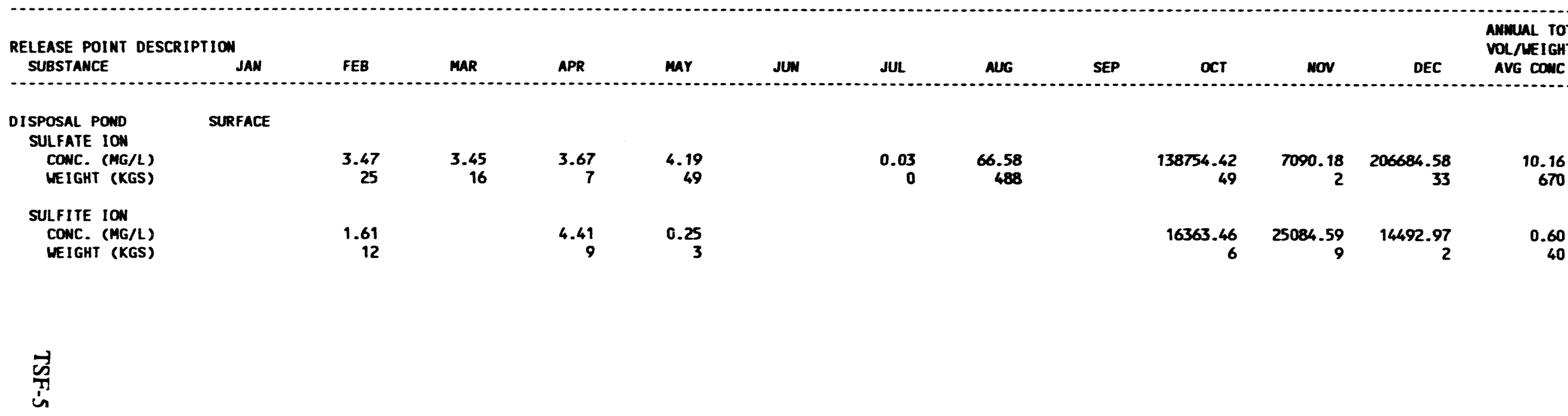


RPT 227-I

FOR JAMUARY THROUGH DECEMBER 1992

TSF WATER USAGE AND DISPOSAL SUMMURY

(ALL VOLUMES = MEAREST THOUSAND LITERS)

ANMUAL TOT

VOL/NEIGHT

WELL OR BLDG. NO./

JAN FEB MAR APR MAY

JUL

AUG

SEP

OCT

Nov DEC AVG CONC

WATER DATA

\begin{tabular}{|c|c|c|c|c|c|c|c|c|c|c|c|c|c|c|c|}
\hline $\begin{array}{l}612 \text { WATER } \\
613 \text { WATER }\end{array}$ & $\begin{array}{l}\text { PUAPED } \\
\text { PUMPED }\end{array}$ & $\begin{array}{l}\text { (L) } \\
\text { (L) }\end{array}$ & $\begin{array}{r}222 \\
6,678\end{array}$ & $\begin{array}{l}4.524 \\
1.536\end{array}$ & $\begin{array}{r}231 \\
8,097\end{array}$ & $\begin{array}{r}167 \\
8,097\end{array}$ & $\begin{array}{r}231 \\
7,192\end{array}$ & $\begin{array}{l}1,402 \\
7,567\end{array}$ & $\begin{array}{l}2,303 \\
3,227\end{array}$ & $\begin{array}{r}10,516 \\
7,715\end{array}$ & $\begin{array}{l}3,519 \\
2,664\end{array}$ & $\begin{array}{r}4,221 \\
631\end{array}$ & $\begin{array}{l}3,774 \\
1,812\end{array}$ & $\begin{array}{l}4,474 \\
3,654\end{array}$ & $\begin{array}{l}35,582 \\
58,871\end{array}$ \\
\hline MATER & PUMPED ( & (L) & 6,900 & 6,060 & 8,328 & 8,264 & 7.423 & 8,969 & 5,530 & 18,230 & 6,183 & 4,852 & 5,586 & 8,128 & 94,453 \\
\hline
\end{tabular}

MATER DISPOSED

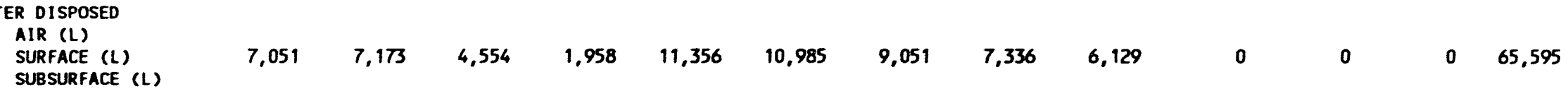

SUBSURFACE (L)

623 BUILD VOL (L) $\quad 3,678 \quad 37,298$

BIOCHEMICAL OXYGEN DEMAND (BOD)

$\begin{array}{lll}\text { RAY CONC. (MG/L) } 151.0 & 178.7\end{array}$

$\begin{array}{ll}18.2 & 22.3 \\ 87.9 & 87.5\end{array}$

X REMOVED

22.3
87.5

3,774

3,785

416

$4,982 \quad 5,167 \quad 5,788$

55

6,261

5,708

5,924

82.837

DISSOLVED OXYGEN (DO)

RAW CONC. (MG/L)

FINAL CONC. (MG/L)

$\begin{array}{ll}4.6 & 5.2 \\ 6.3 & 6.1\end{array}$

INCREASED FACTOR

146.3
22.3
84.8

105.0

$\begin{array}{ll}16.3 & 36.0\end{array}$

89.0
17.0
80.

$104.0 \quad 120.0 \quad 145.0$

$84.5 \quad 73.7$

80.9

18.0
82.7

21.0
82.5

145.0
19.5

76.3

16.0

82.

82.5
22.3
73.0

$75.0 \quad 117.5$

84.8

SETTLEABLE SOLIDS (SS)

RAU CONC. (ML/L)

FINAL CONC. (ML/L)

$7.3 \quad 92.7$
0.01

X REMOVED

$0.01 \quad 0.02$

PH FINAL

8.08.

TOTAL SEWAGE(L)

$3,678 \quad 37,298$

TOTAL WATER DISPOSED(L) $\quad 10,729 \quad 44,471$

\begin{tabular}{|c|c|c|c|c|c|c|c|c|c|c|}
\hline $\begin{array}{l}4.1 \\
5.6 \\
0.4\end{array}$ & $\begin{array}{r}5.7 \\
5.4 \\
-0.1\end{array}$ & $\begin{array}{l}3.0 \\
5.0 \\
0.7\end{array}$ & $\begin{array}{l}3.0 \\
5.0 \\
0.7\end{array}$ & $\begin{array}{l}3.0 \\
4.0 \\
0.3\end{array}$ & $\begin{array}{l}3.0 \\
4.0 \\
0.3\end{array}$ & $\begin{array}{l}2.8 \\
5.1 \\
0.8\end{array}$ & $\begin{array}{l}3.1 \\
5.2 \\
0.7\end{array}$ & $\begin{array}{l}4.7 \\
6.0 \\
0.3\end{array}$ & $\begin{array}{l}6.1 \\
7.0 \\
0.1\end{array}$ & $\begin{array}{l}4.0 \\
5.4 \\
0.3\end{array}$ \\
\hline $\begin{array}{l}7.1 \\
0.10 \\
98.6\end{array}$ & $\begin{array}{r}8.1 \\
0.10 \\
98.8\end{array}$ & $\begin{array}{r}9.0 \\
0.10 \\
98.9\end{array}$ & $\begin{array}{r}7.0 \\
0.29 \\
95.9\end{array}$ & $\begin{array}{r}9.0 \\
0.11 \\
98.8\end{array}$ & $\begin{array}{r}9.0 \\
0.98 \\
89.1\end{array}$ & $\begin{array}{l}7.1 \\
0.06 \\
99.2\end{array}$ & $\begin{array}{r}5.8 \\
0.05 \\
99.1\end{array}$ & $\begin{array}{r}6.6 \\
0.03 \\
99.5\end{array}$ & $\begin{array}{r}4.1 \\
0.02 \\
99.5\end{array}$ & $\begin{array}{l}14.4 \\
0.16 \\
98.9\end{array}$ \\
\hline 7.9 & 7.7 & 7.6 & 8.0 & 8.0 & 8.0 & 7.5 & 8.0 & 7.8 & 7.9 & 7.9 \\
\hline 3,774 & 3,785 & 416 & 4,982 & 5,167 & 5,788 & 55 & 6,261 & 5,708 & 5,924 & 82,837 \\
\hline 8,328 & 5.744 & 11.773 & 15,967 & 14,218 & 13.124 & 6,184 & 6,261 & 5,709 & 5,924 & 148,431 \\
\hline
\end{tabular}


PAGE:

SCHED. NO. INRPTO10
IDAHO OPERATIOWS OFFICE

UNITED STATES DEPARTMENT OF ENERGY

19- JUN-93

INEL NONRADIOLOGICAL MASTE MUNAGEMENT INFORMATION SYSTEM

FOR JAMUARY THROUGH DECEMBER 1992

TSF WATER USAGE AND DISPOSAL SUMMARY

RPT 227-I

TSF

(ALL VOLUNES = NEAREST THOUSAND LITERS)

ANWUAL TOT

VOL/WEIGHT WELL OR BLDG. NO./
SEWAGE BLDG. NO.

SENAGE BLDG. NO.

ACCOUNTABILITY (\%)

733.9

100.0

69.5

158.6

257.1

$72.0 \quad 100.0$

129.0

102.2

72.9 
PAGE: 1

SCHED. NO. INRPTOII
IOAHO OPERATIONS OFFICE

19- JUN-93

UNITED STATES DEPARTMENT IF ENERGY

FOR JANUARY THROUGH DECEMBER 1992

TSF FUEL OIL USAGE AND STACK EFFLUENTS SUMmARY

RPT 228-1

TSF

(ALL VALUES = MEAREST THOUSAND)

\begin{tabular}{|c|c|c|c|c|c|c|c|c|c|c|c|c|c|}
\hline $\begin{array}{l}\text { FUEL OIL TYPE } \\
\text { EFFLUENTS }\end{array}$ & JAN & FEB & MAR & APR & MAY & JUN & JUL & AUG & SEP & OCT & NOV & DE : & $\begin{array}{l}\text { ANNUAL } \\
\text { TOTALS }\end{array}$ \\
\hline \multicolumn{14}{|l|}{ TYPE 2} \\
\hline VOLUME (LITERS) & 319 & 244 & 208 & 136 & 0 & & & & & 27 & 148 & 290 & 1,372 \\
\hline SO2 (KGS) & 2 & 1 & 1 & 1 & 0 & & & & & 0 & 1 & 2 & \\
\hline \multirow{2}{*}{\multicolumn{14}{|c|}{$\begin{array}{l}\text { NOX (KGS) } \\
\text { PARTICULATES (KGS) }\end{array}$}} \\
\hline & & & & & & & & & & & & & \\
\hline \multicolumn{14}{|l|}{ GRAND TOTALS: } \\
\hline VOLUME (LITERS) & 319 & 244 & 208 & 136 & 0 & & & & & 27 & 148 & 290 & 1,372 \\
\hline SO2 (KGS) & 2 & 1 & 1 & 1 & 0 & & & & & 0 & 1 & 2 & \\
\hline $\begin{array}{l}\text { NOX (KGS) } \\
\text { PARTICULATES (KG }\end{array}$ & & & & & & & & & & & & & \\
\hline
\end{tabular}




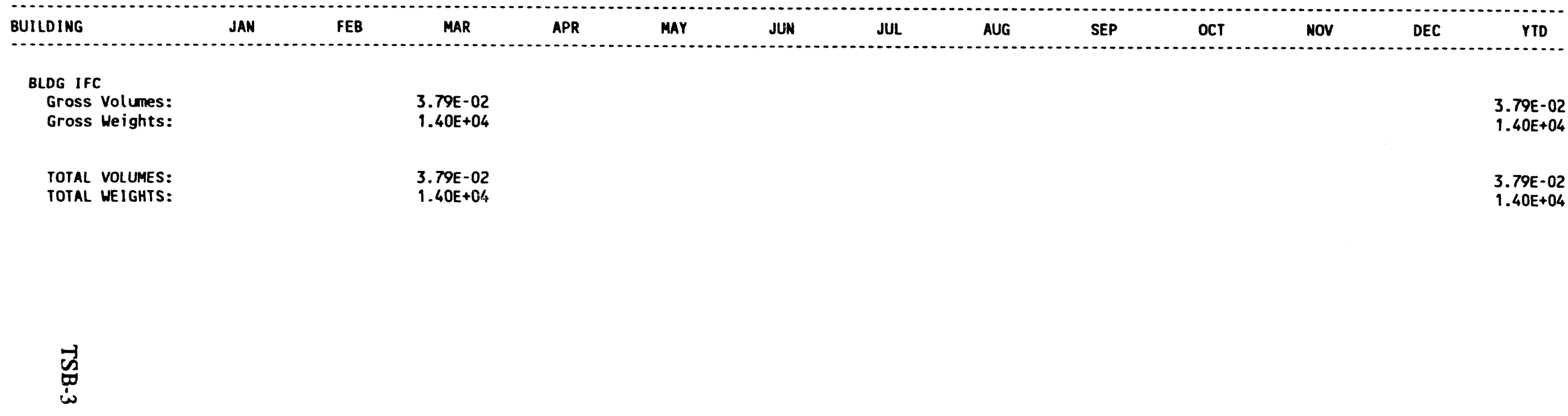

NOTE: VOLUME REPORTED IN CUBIC METERS; WEIGHT REPORTED IN GRAMS DETAILS MAY NOT ADD UP TO TOTALS BECAUSE OF ROUNDING 


\section{WILLOW CREEK BUILDING, 1992 DETAIL DATA}

Report 10-Hazardous Waste Generated By WCB For January-December 1992

WCB-3 
HAZARDOUS WASTE GENERATED BY WCB

RPT 10-I

FOR January - December 1992

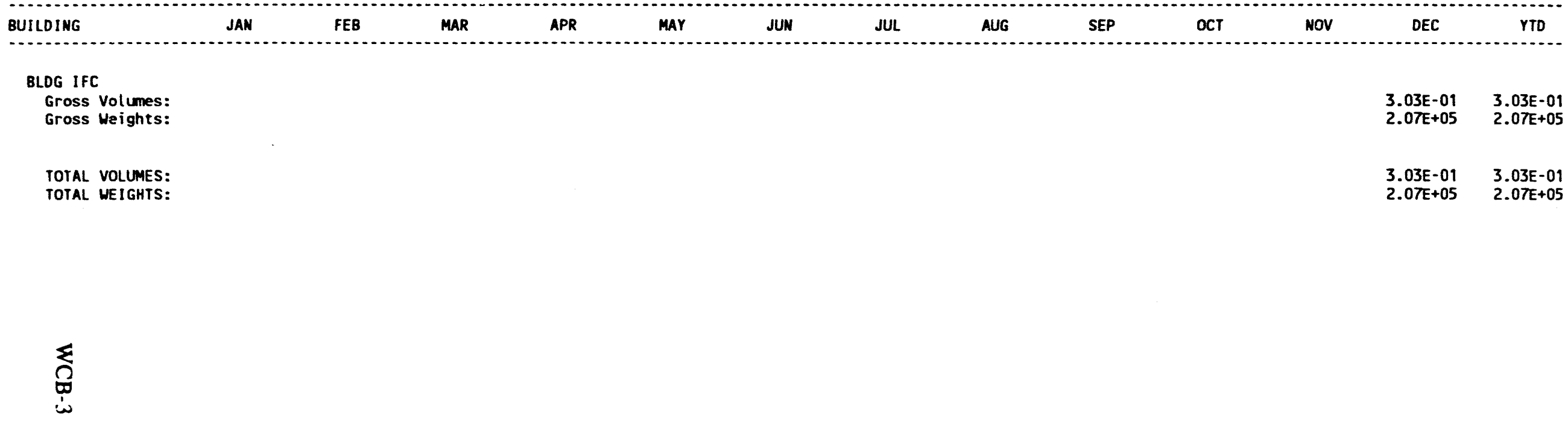


WASTE EXPERIMENTAL REDUCTION FACILITY, 1992 DETAIL DATA

Report 2411-Mixed Waste Generated By WER For January-December 1992 WER-3 
MIXED WASTE GENERATED BY MER

FOR January - December 1992

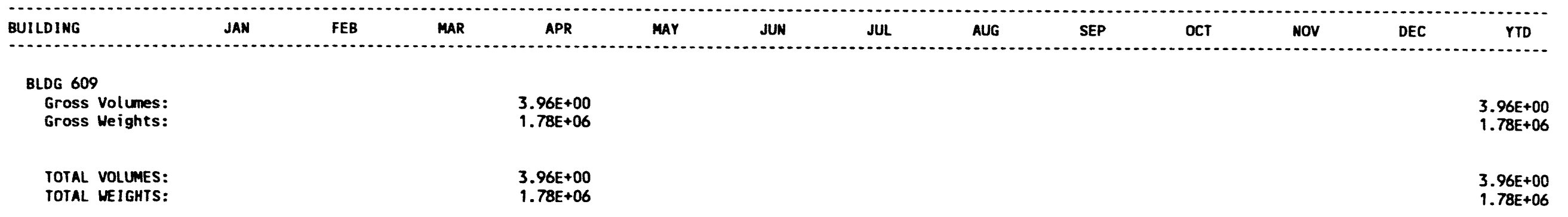


RADIOACTIVE WASTE MANAGEMENT COMPLEX, 1992 DETAIL DATA

Report 256-WMC Landfill Solid Disposed Waste Summary ............WMC-3

Report 257-WMC Water Usage And Disposal Summary For January

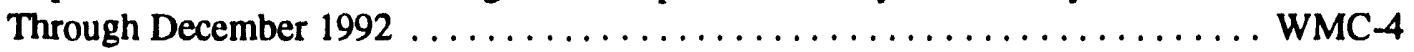

Report 2511-Mixed Waste Generated By WMC For January-December

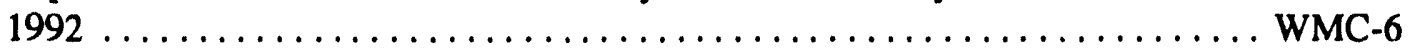


WATER REACTOR RESEARCH TEST FACILITY-TEST AREA NORTH, 1992 DETAIL DATA

Report 265-WRF Liquid Disposed Substances Summary ............ WRF-3

Report 265-WRF Release Point And Liquid Disposed Substances Summary . . . WRF-4

Report 267-WRF Water Usage And Disposal Summary For January

Through December $1992 \ldots \ldots \ldots \ldots \ldots \ldots \ldots \ldots \ldots \ldots \ldots \ldots$ WRF-6

Report 268 - WRF Fuel Oil Usage And Stack Effluents Summary . . . . . . . WRF-8

Report 2610-Hazardous Waste Generated By WRF For January-December

1992

WRF-9 


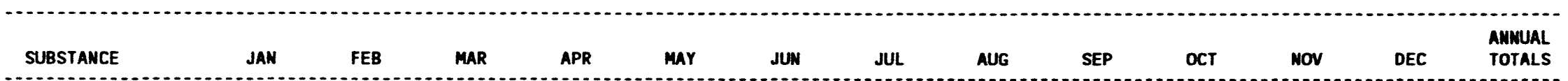

CHLORIDE ION

WEIGHT (KGS)

151

CYCLOHEXYLAMINE

WEIGHT (KGS)

ETHYLENEDIAMINE TETRAACETIC ACID WEIGHT (KGS) 0

JANITORIAL SUPPLIES VEIGHT (KGS)

MORPHOL INE WEIGHT (KGS)

POTASSIUM HYDROXIDE WEIGHT (KGS)

SOOIUM ION VEIGHT (KGS) SOOIUM LIGNOSULFONATE VEIGHT (KGS)

SULFATE IOI

WEIGHT (KGS)

SULFITE ION

NEIGHT (KGS)

UNKNOWM

MEIGHT (KGS)

11

358

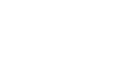

0

4 


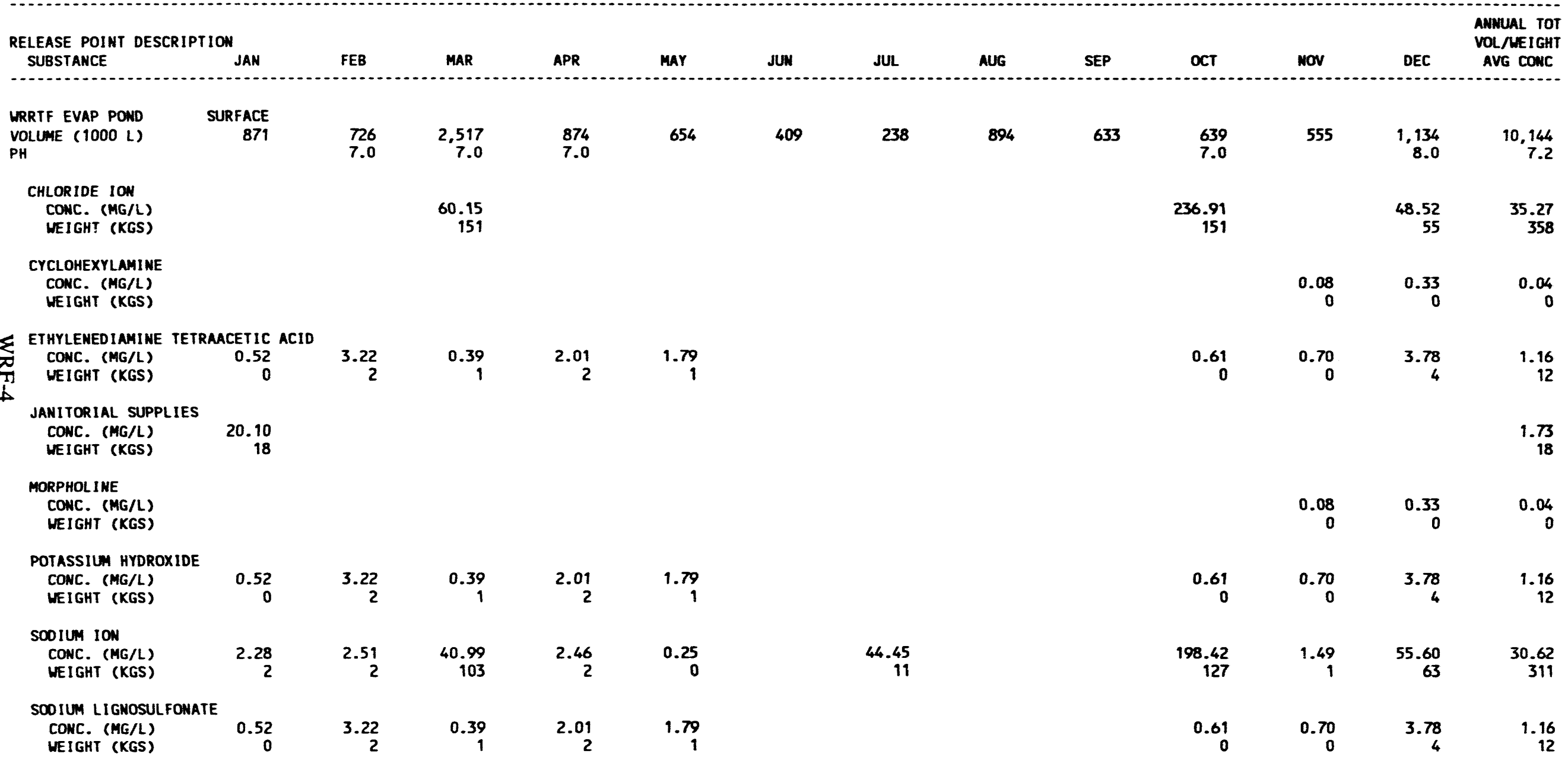




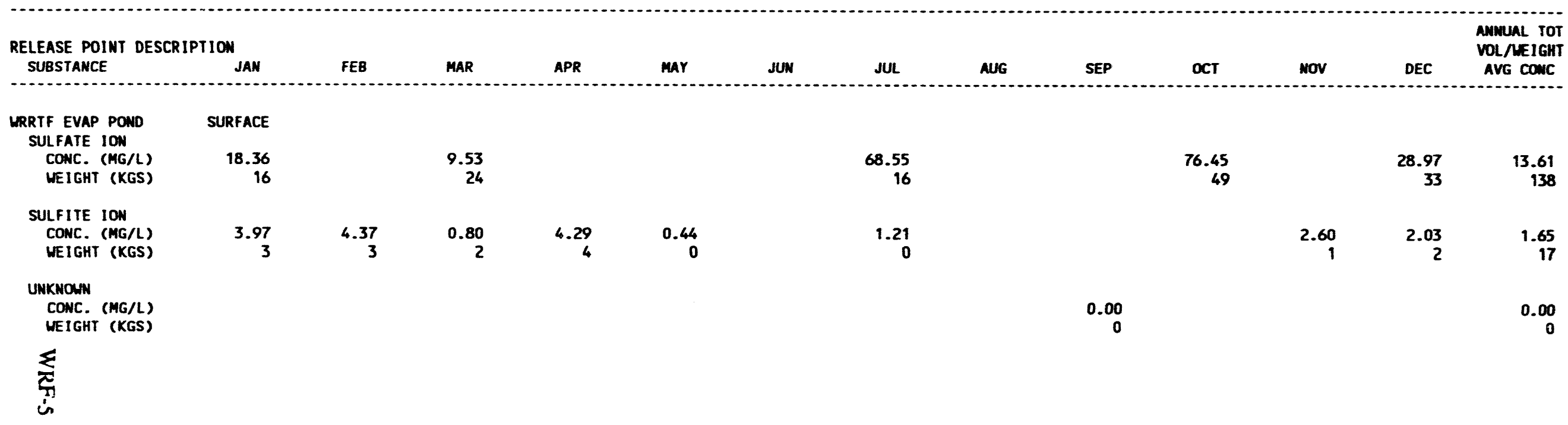


PAGE : 1

SCHED. NO. INRPTO1O

IDAHO OPERATIONS OFFICE

UNITED STATES DEPARTMENT OF EMERGY

19- JuW-93

INEL NONRADIOLOGICAL WASTE MAMAGEMENT INFORMATION SYSTEM

FOR JANUARY THROUGH DECEMBER 1992

WRF MATER USAGE AND DISPOSAL SUMHARY

RPT 267-I

(ALL VOLUMES = NEAREST THOUSAMD LITERS)

URF

ANMUAL TOT ANMUAL TOT WELL OR BLDG. NO. SEWAGE BLDG. NO. JAN

FEB

MAR APR MAY JUW

JUL
AUG SEP OCT Nov DEC AVG CONC

WATER DATA

WRF WATER PUMPED (L)

\begin{tabular}{|c|c|c|c|c|c|c|c|c|c|c|c|c|}
\hline 1,122 & 1,209 & 3,107 & 1,067 & 798 & 500 & 291 & 1,091 & 773 & 776 & 676 & 1,384 & 12,795 \\
\hline
\end{tabular}

WATER PUMPED (L)

871

726

AIR (L)

SURFACE (L)

871

2,517

874

654

409

238

894

633

639

555

1,134

10,144

SEMAGE DATA

BUILD VOL (L)

BIOCHEMICAL OXYGEN DEMAND (BOD)

RAW CONC. (MG/L)

FINAL CONC. (MG/L)

$X$ REMOVED

DISSOLVED OXYGEN (DO)

RAW CONC. (MG/L)

FINAL CONC. (MG/L)

INCREASED FACTOR

SETTLEABLE SOLIDS (SS)

RAW CONC. (ML/L)

FINAL CONC. (ML/L)

\% REMOVED

PH FINAL 
FOR JAMUARY THROUGH DECEMBER 1992

(ALL VOLUMES = MEAREST THOUSANO LITERS)

\section{JAN}

FEB

MAR

APR

MAY

JUN

JUL

AUG

SEP

OCT

NOV DEC AVG CONC

643 BUILD VOL (L)

87

311

BIOCHEMICAL OXYGEN DEMAND (BOO)

RAW CONC. (MG/L)

FIMAL CONC. (MG/L)

X REMOVED

DISSOLVED OXYGEN (DO)

RAL CONC. (MG/L)

FINAL CONC. (MG/L)

INCREASED FACTOR

SETTLEABLE SOLIDS (SS)

RAH CONC. (ML/L)

FINAL CONC. (ML/L)

× REMOVED

\section{PH FINAL}

TOTAL SEWAGE(L)

TOTAL MATER DISPOSED(L)

1,095

ACCOUNTABILITY (x)

97.6

726

2,517

60.0

81.0
654

2.3


PAGE: 1

SCHED. NO. IMRPTO11

IDAHO OPERATIONS OFFICE

19-JUN-93

DLOGICAL MASTE MAMAGEMENT INFORMATION SYSTEM

FOR JAMUARY THROUGH DECEMBER 1992

URF FUEL OIL USAGE AND STACK EFFLUENTS SUMARTY

RPT 268-I

(ALL VALUES = MEAREST THOUSAND)

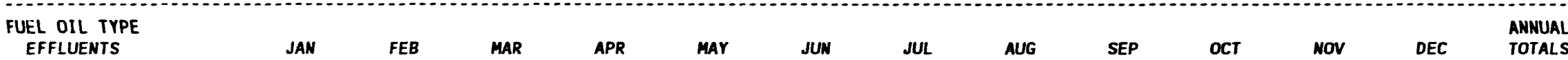

TYPE 2

VOLUME (LITERS)

SO2 (KGS)

NOX (KGS)

PARTICULATES (KGS)

GRAND TOTALS:

VOLUME (LITERS)

SO2 (KGS)

NOX (KGS)

PARTICULATES (KGS) 
HAZARDOUS MASTE GENERATED BY URF FOR January - December 1992
RPT 2610-1

WRF

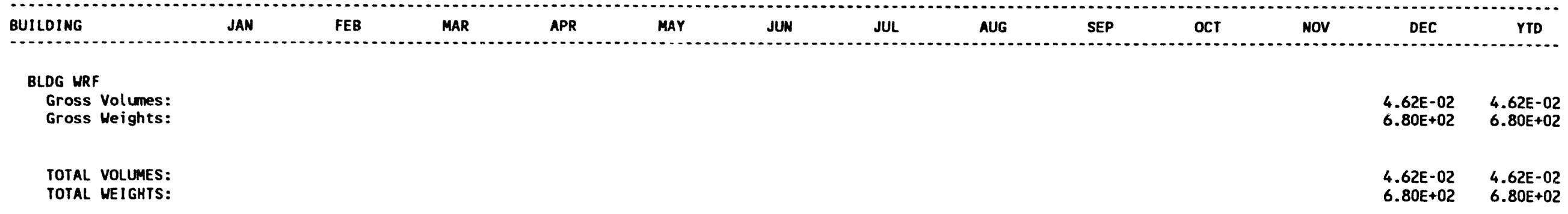


|

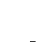

